\title{
U. S. Uranium Mining Industry: Background Information on Economics and Emissions
}

\author{
G. A. Bruno \\ J. A. Dirks \\ P. O. Jackson \\ J. K. Young
}

March 1984

\section{Prepared for}

the Environmental Protection Agency

under Related Services Agreement

with the U.S. Department of Energy

under Contract DE-AC06-76RLO 1830

Pacific Northwest Laboratory

Operated for the U.S. Department of Energy

by Battelle Memorial Institute 


\begin{abstract}
DISCLAIMER
This report was prepared as an account of work sponsored by an agency of the United States Government. Neither the United States Government nor any agency thereof, nor any of their employees, makes any warranty, express or implied, or assumes any legal liability or responsibility for the accuracy, completeness, or usefulness of any information, apparatus, product, or process disclosed, or represents that its use would not infringe privately owned rights. Reference herein to any specific commercial product, process, or service by trade name, trademark, manufacturer, or otherwise, does not necessarily constitute or imply its endorsement, recommendation, or favoring by the United States Government or any agency thereof. The views and opinions of authors expressed herein do not necessarily state or reflect those of the United States Government or any agency thereof.
\end{abstract}

\title{
PACIFIC NORTHWEST LABORATORY operated by BATTELLE for the UNITED STATES DEPARTMENT OF ENERGY under Contract DE-AC06-76RLO 1830
}

\begin{tabular}{|c|c|}
\hline \multicolumn{2}{|c|}{$\begin{array}{c}\text { National Technical Information Service } \\
\text { United States Department of Commerce } \\
5285 \text { Port Royal Road } \\
\text { Springfield, Virginia } 22161\end{array}$} \\
\hline \multicolumn{2}{|c|}{$\begin{array}{l}\text { NTIS Price Codes } \\
\text { Microfiche A01 }\end{array}$} \\
\hline \multicolumn{2}{|c|}{ Printed Copy } \\
\hline Pages & $\begin{array}{l}\text { Price } \\
\text { Codes }\end{array}$ \\
\hline 001-025 & $\mathrm{A} 02$ \\
\hline $026-050$ & $\mathrm{~A} 03$ \\
\hline 051-075 & A04 \\
\hline $076-100$ & $\mathrm{~A} 05$ \\
\hline $101-125$ & A06 \\
\hline $126-150$ & $\mathrm{~A} 07$ \\
\hline $151-175$ & $\mathrm{~A} 08$ \\
\hline $176-200$ & A09 \\
\hline $201-225$ & $A 010$ \\
\hline $226-250$ & A011 \\
\hline $251-275$ & $\mathrm{~A} 012$ \\
\hline $276-300$ & A013 \\
\hline
\end{tabular}


PNL -5035

UC-2,11,51

U.S. URANIUM MINING INDUSTRY:

BACKGROUND INFORMATION ON

ECONOMICS AND EMISSIONS

\author{
G. A. Bruno \\ J. A. Dirks \\ P. 0. Jackson \\ J. K. Young
}

March 1984

\begin{abstract}
Prepared for
the Environmental Protection Agency under Related Services Agreement

with the U.S. Department of Energy

under Contract DE-AC06-76RLO 1830
\end{abstract}

Pacific Northwest Laboratory

Richland, Washington 99352 


\section{CONTENTS}

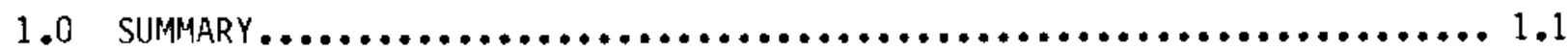

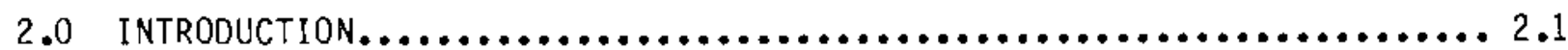

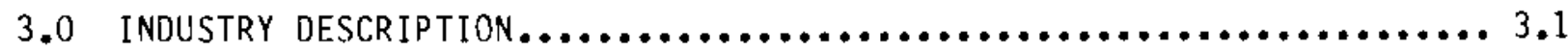

3.1 COMMODITY CHARACTERISTICS $\ldots \ldots \ldots \ldots \ldots \ldots \ldots \ldots \ldots \ldots \ldots \ldots \ldots . . . \ldots \ldots$

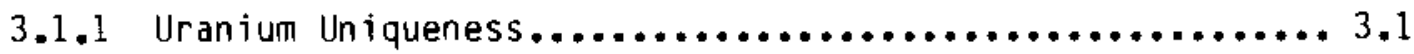

3.1 .2 Terminology.................................... 3.6

3.1 .3 Chemistry.................................... 3.7

3.2 DEPOSIT-TYPE DESCRIPTIONS $\ldots \ldots \ldots \ldots \ldots \ldots \ldots \ldots \ldots \ldots \ldots \ldots \ldots . . . \ldots . . . \ldots$

3.3 MINING PROCESSES $\ldots \ldots \ldots \ldots \ldots \ldots \ldots \ldots \ldots \ldots \ldots \ldots \ldots \ldots \ldots \ldots, \ldots, \ldots \ldots \ldots$

3.3.1 Underground Mining................................... 3.13

3.3 .2 Open-Pit Mining................................... 3.15

3.3.3 Solution Mining.................................. 3.16

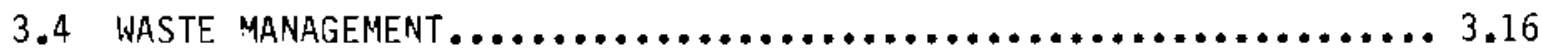

3.4.1 Airborne Waste................................... 3.16

3.4 .2 Liquid Wastes.................................... 3.18

3.4 .3 Solid Wastes...................................... 3.19

3.4.4 Reclamation........................................ 3.19

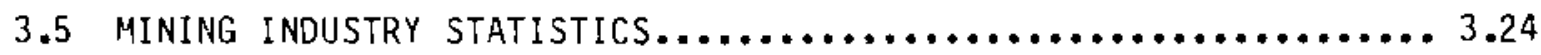

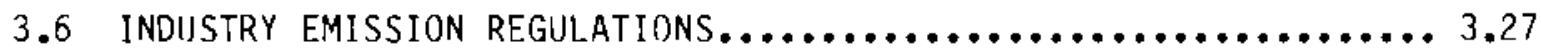

3.6 .1 Underground Exposure............................. 3.27

3.6.2 Emission to Atmosphere........................... 3.29

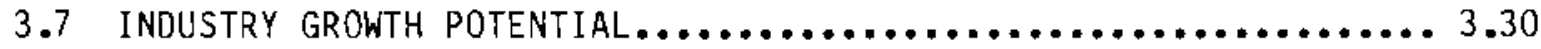

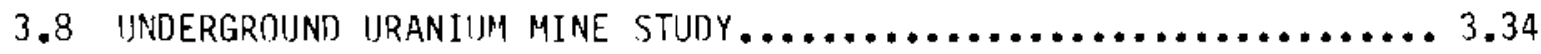

3.8.1 Study Mine Selection and Background.................. 3.34 
3.8.2 Study Mine Site Characterization................. 3.40

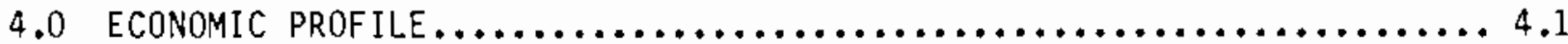

4.1 DEMAND........................................ 4.1

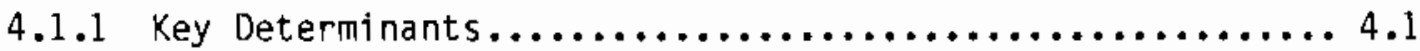

4.1 .2 Trends in Energy Consumption.................... 4.5

4.1 .3 Uranium Pricing.............................. 4

4.1 .4 Elasticity of Demand.......................... 46

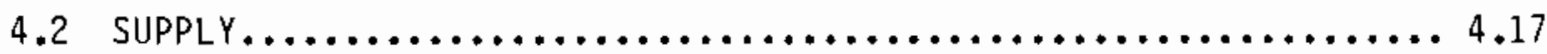

4.2 .1 Key Determinants.......................... 4.17

4.2.2 Interindustry Organization and Market............. 4.20

4.2.3 Trends in Production and Supply.................. 4.25

4.2.4 Facility Utilization--Operating Capacity, Production Lifetime.................................4.28

4.2.5 Productivity and Employment Trends............... 4.32

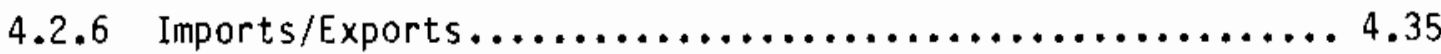

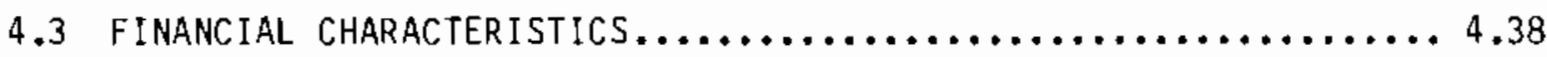

4.3 .1 Capital Requirements........................ 4.38

4.3 .2 Financial Data.............................. 4.42

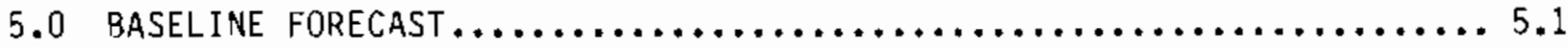

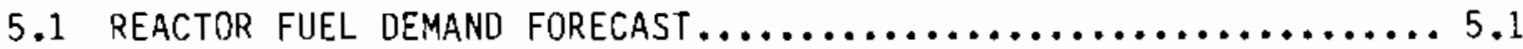

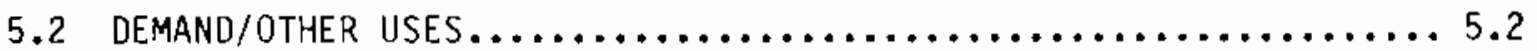

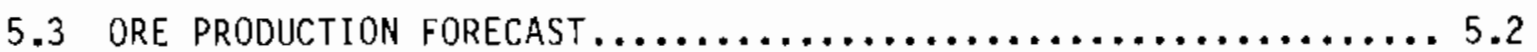

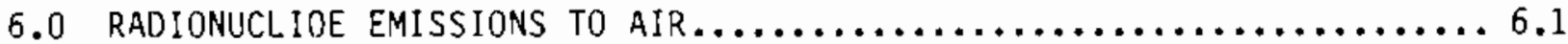

6.1 TECHNIQUES FOR MONITORING ${ }^{222}$ Rn EMISSIONS FROM UNDERGROUND URANIUM MINES ................................. 6.1

6.1 .1 Radon Measurements.......................... 6.3

6.1.2 Calibration of Radon Monitoring Instruments..........6.15 
6.1 .3 Vent Flow Measurements......................... 6.16

6.2 EMISSIONS CONTROL DESCRIPTIONS $\ldots \ldots \ldots \ldots \ldots \ldots \ldots \ldots \ldots \ldots \ldots . .17$

6.2 .1 Stacks $\ldots \ldots \ldots \ldots \ldots \ldots \ldots \ldots \ldots \ldots \ldots \ldots \ldots \ldots \ldots \ldots, 6.18$

$6.3222_{R n}$ EMISSIONS TO AIR FROM UNDERGROUND URANIUM MINES $\ldots \ldots \ldots \ldots 6.23$

6.3 .1 Methodology of Calculations...................... 6.23

6.3 .2 Calculation Process $\ldots \ldots \ldots \ldots \ldots \ldots \ldots \ldots \ldots \ldots \ldots \ldots .6 .28$

6.3 .3 Results and Discussion.......................... 6.30

6.3 .4 Conclusions................................... 6.30

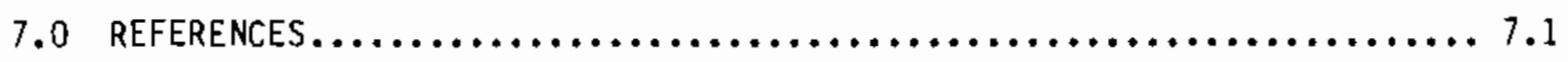

APPENDIX A GLOSSARY OF MINING TERMS............................ 1

APPENDIX B PRODUCTIVITY FACTOR CALCULATION...................... B.1

APPENDIX $C$ STUDY MINE DATA SHEETS $. \ldots \ldots \ldots \ldots \ldots \ldots \ldots \ldots \ldots \ldots \ldots \ldots . . \ldots \ldots$

APPENDIX D STUDY MINE LOCATION AND DATA MAPS $\ldots \ldots \ldots \ldots \ldots \ldots \ldots \ldots \ldots . .1$

APPENDIX E ASSOCIATED MINE FACILITIES DESCRIPTIONS................. E.1

APPENDIX $F$ PRESIDENT REAGAN'S STATEMENT OF NUCLEAR ENERGY POLICY........ F.I

APPENDIX G FINANCIAL ANALYSIS $\ldots \ldots \ldots \ldots \ldots \ldots \ldots \ldots \ldots \ldots \ldots \ldots \ldots \ldots \ldots \ldots \ldots \ldots$ 


\section{•




\section{FIGURES}

3.1 Grade of Uranium Ore Processed............................. 3.5

3.2 Uranium Prices Compared to Uranium Production Costs and to Prices of Other Commodities................................... 3.6

3.3 Schematic Cross-Section of a Wyoming-Type Role Uranium Deposit....... 3.12

4.1 History of $U_{3} 0_{8}$ Prices ............................... 4.15

\section{TABLES}

3.1 Ancient Versus Modern Metals and Fuels...................... 3.2

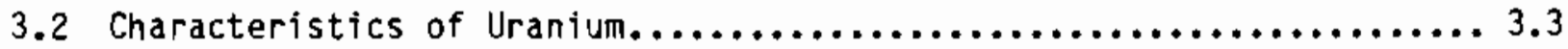

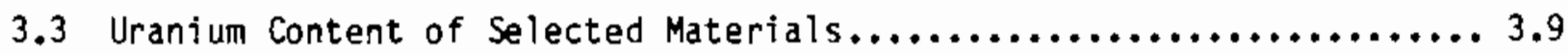

3.4 Most Common Uranium Minerals............................ 3.10

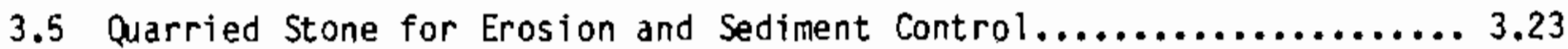

3.6 Distribution of 1982 Uranium Production by Mining Method.......... 3.25

3.7 Distribution of 1982 Uranium Production by State.....................25

3.8 Uranium Ore Produced from Mines and Received at Mills and Buying Stations ............................................. 3.26

3.9 Ore Processed and Concentrate Produced in 1982................. 3.26

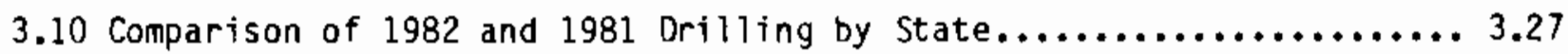

3.11 Number of Reactors Starting Commercial Operation..................... 31

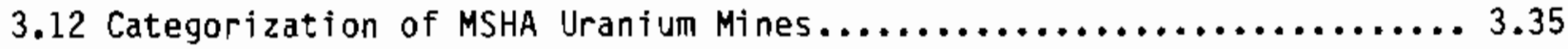

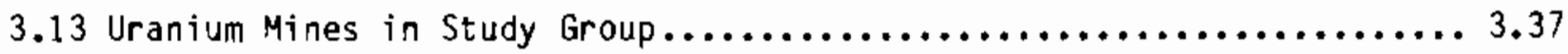

3.14 Employee-hours, FTE Workers, and Estimated Production...............41

3.15 Average Persons Per Household............................ 3.43

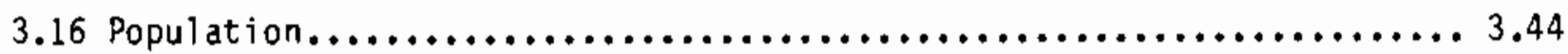

3.17 Indian Population and Total Relocation Costs....................45 


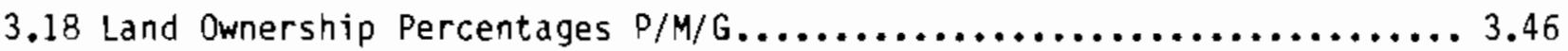

3.19 Estimated value of Private Land................................ 39

3.20 Aggregate Assessment/Sales Price Ratios...................... 3.51

3.21 Local Real Estate Estimates for Grazing and Residential Property.... 3.52

3.22 Annualized Cost of Controlling Land to $2 \mathrm{~km}, \ldots \ldots \ldots \ldots \ldots \ldots \ldots \ldots . .54$

4.1 Uncertainties Affecting Demand Projections $\ldots \ldots \ldots \ldots \ldots \ldots \ldots \ldots \ldots . .3$

4.2 Projections of Installed Nuclear Capacity Presented at Annual Uranium Industry Seminar .................................. 4.6

4.3 Current Projection of Installed Nuclear and Total Electric Utility Capacity by DOE/EIA ..................................... 4.7

4.4 Previous Projection of Installed Nuclear and Total Electric Generating Capacity by ERDA $\ldots \ldots \ldots \ldots \ldots \ldots \ldots \ldots \ldots \ldots \ldots \ldots \ldots, \ldots \ldots \ldots, 7$

4.5 Circulars 3 and 4: Price Schedule for Colorado Plateau Uranium From Carnotite and Roscoelite Ores............................. 4.9

4.6 Circular 5 and Circular 6: Schedule of Prices for Uranium Ore......4.10

4.7 Average value of Ore........................................ 42

4.8 AEC Domestic Concentrate Purchases: Amount, Cost and Average Price.. 4.13

4.9 Historical Exchange Values............................... 4.14

4.10 Current Nuclear Fuel Cycle Activities $. . \ldots \ldots \ldots \ldots \ldots \ldots \ldots \ldots \ldots . .21$

4.11 Uranium Ore Produced from Mines and Received at Mills and Buying

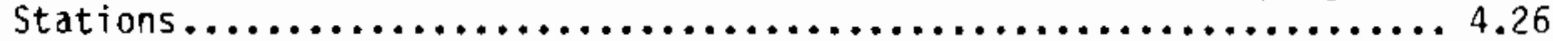

4.12 U.S. Uranium Processing Plants: Operating as of January 1, 1983....4.30

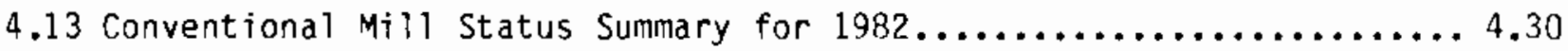

4.14 Average New Mine....................................... 4.31

4.15 Estimates of Labor Productivity in Uranium Mining................ 4.33

4.16 Changes in Output Per Man in Open-Pit and Underground Mines........4.34

4.17 Employment in the Uranium Industry........................... 45

4.18 Deliveries of Uranium to DOE Enrichment Plants by Domestic Customers 4.36 
4.19 U.S. Imports and Exports of Uranium for Commercial Uses........... 4.39

4.20 Capital Investment for the Domestic Uranium Industry............4.41

4.21 Financial Information for Three Uranium-Mining Companies.........4.43

5.1 Annual U.S. Uranium Demand in Tons $\mathrm{U}_{3} \mathrm{O}_{8} \times 10^{3} \ldots \ldots \ldots \ldots \ldots \ldots \ldots . \ldots . \ldots$

5.2 Annual U.S. Uranium Production in Tons $v_{3} 0_{8} \times 10^{3} \ldots \ldots \ldots \ldots \ldots \ldots$

5.3 Annual Ore Production................................. 5.3

6.1 Manufacturers of Radon Analysis Equipment................... 6.6

6.2 Exhaust Borehole Stack Cost Final Estimate.................... 6.20

6.3 Increased Cost of Production Resulting from Adding Ventilation

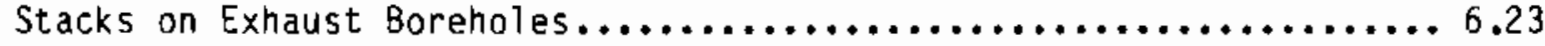

6.4 Summary of Radon Emissions from Underground Mine Vents............ 6.24

6.5 Current Status of Mines Monitored in 1978-1979 Survey of Jackson

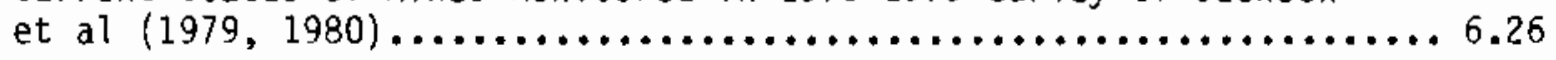

6.6 Uranium Ore Produced from Underground Mines and Fractional

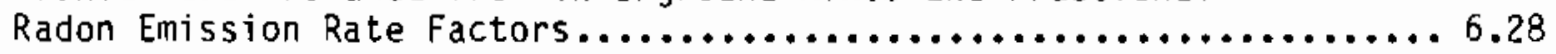

6.7 Measured and Predicted ${ }^{222}$ Rn Emission Rates................... 6.31 


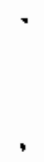




\subsection{SUMMARY}

A review of the U.S. uranium mining industry has reveaied a generally depressed industry situation. The $1982 \mathrm{U}_{3} \mathrm{O}_{8}$ production from both open-pit and underground mines declined to 3,800 and 6,300 tons respectively with the underground portion representing $46 \%$ of total production.

U.S. exploration and development has continued downward in 1982. Many programs have been curtailed or abandoned, drilling has declined, and total industry expenditures have dropped. Of 15 major oil companies active early in 1982, only 7 remain in the uranium business and at reduced levels. The total number of conventional uranium production sources in 1982 was down to 139 underground mines and 24 open-pit mines. Drilling was down 56\% from 1981 to 61 million feet. Total 1982 industry capital investment declined to $\$ 163$ million or $39 \%$ of 1981 expenditures.

Employment in the mining and milling sectors has dropped $31 \%$ and $17 \%$ respectively in 1982. Capacity utilization at mills was 45\% in 1982 with only 14 mills still operating by year end out of 20 at the beginning of the year. Many mines were at $50 \%$ capacity or on standby status. The depressed nature of the U.S. uranium mining industry can be attributed mainly to a weakened demand for uranium fuel and a low comodity price caused by oversupply.

Representative forecasts were developed for reactor fuel demand and $\mathrm{U}_{3} \mathrm{O}_{8}$ production for the years 1983 and 1990. Reactor fuel demand is estimated to increase from 15,900 tons to 21,300 tons $U_{3} 0_{8}$ respectively. $U_{3} 0_{8}$ production, however, is estimated to decrease from 10,600 tons to 9,600 tons respectiveiy. This projected decrease in $\mathrm{U}_{3} \mathrm{O}_{8}$ production can be attributed to a predicted increase in imports and a predicted continuation of inventory liquidation. In 1983, imports are estimated at $28 \%$ of uranium used in the U.S., increasing to an estimated $37.5 \%$ in 1990 .

A field examination was conducted of 29 selected underground uranium mines that represent $84 \%$ of the 1982 underground production. Data was gathered regarding population, land ownership and private property valuation. Out to $2 \mathrm{~km}$ total population was 618 persons. Average land ownership was $22 \%$ private, $17 \%$ mining company and $61 \%$ government. The value of 1 and and structures was 
estimated at $\$ 9,378,270$ out to $2 \mathrm{~km}$. The annual cost to control land out to $2 \mathrm{~km}$ was estimated at $\$ 3,012,000$ equivalent to a $\$ 0.29$ per pound $\mathrm{J}_{3} \mathrm{O}_{8}$ increase in the cost of production based on 1982 production.

An andysis of the increased cost to production resulting from the installation of 20-meter high exhaust borehole vent stacks was conducted. For a most likely case, increased cost would be $\$ 0.493$ to $\$ 0.881$ per ton of ore or $\$ 0.062$ to $\$ 0.242$ per pound of $\mathrm{U}_{3} \mathrm{O}_{8}$.

An assessment was made of the current and future ${ }^{222}$ Rn emission levels for a group of 27 uranium mines. It is shown that ${ }^{222}$ Rn emission rates are increasing from 10 individual operating mines through 1990 by 1.2 to 3.8 times. But for the group of 27 mines as a whole, a reduction of total ${ }^{222_{R n} \text { emissions }}$ is predicted due to 17 of the mines being shutdown and sealed. The estimated total ${ }^{222} \mathrm{Rn}$ emission rate for this group of mines will be $105 \mathrm{Ci} / \mathrm{yr}$ by year end 1983 or $70 \%$ of the $1978-79$ measured rate and $124 \mathrm{Cj} / \mathrm{yr}$ by year end 1990 or $83 \%$ of the 1978-79 measured rate. 


\subsection{INTRODUCTION}

As a result of a lawsuit filed by the Sierra Club against the Environmental Protection Agency (EPA) in the state of California on June 16, 1981, a court order has dictated that the EPA publish proposed regulations regarding radionuclide emissions from underground uranium mines. The EPA had earlier listed such emissions as hazardous air pollutants under provisions of the Clean Air Act as amended in 1977. In preparing these proposed emission regulations, the EPA required certain background information of the uranium industry as a whole as well as of specific active and recently inactive uranium mines. This background information aided the EPA in both the development and support of these proposed regulations.

In regards to underground uranium mines, the EPA proposed a radionuclide emission standard which restricts the increase in the annual average concentration of ${ }^{222} 2_{n}$ in any unrestricted area around a mine to 0.2 picocuries per liter. Several methods for meeting this standard were suggested which include:

1. reducing the operating time of the mine,

2. increasing the exhaust borehole vent stacks effective heights, and

3. controlling additional land around the mine vents-out to $2 \mathrm{~km}$. This report, in providing the EPA with essential background information, has basically a four-fold objective:

1. to assess the economic background of the uranium industry and describe its present status,

2. to provide as best as possible a forecast of fuel demand and ore production for the years 1983 and 1990 ,

3. to review emissions data from underground uranium mines; document radon measurement/monitoring techniques; assess the magnitude of emissions from a sample of underground mines using projected ore production data; determine production cost increases resulting froin the proposed vent stack height increase to 20 meters, 
4. and to conduct field surveys to gather data on population densities, land ownership, land values, and site characteristics around selected mines; plus determine production cost increases resulting from the land control option of the proposed regulations.

Chapter 3 begins with an overview of uranium--its commodity and oredeposit characteristics. This is followed by other broad descriptions of the uranium mining industry including mining processes, waste management, regulations, general production statistics plus a statement on the industry's growth potential. Chapter 3 is concluded with a detailed description of the field surveys perfomed on 29 selected mines in our study group. This description includes the production cost analysis involving the land control option.

Chapter 4 provides an economic profile of the uranium industry which is divided into demand, supply, and financial topics. Once the background is soundly developed, fuel demand and ore production forecasts are given in Chapter 5.

Finally, a detailed review of emissions data, resulting in an assessment of the projected magnitude of emissions from a sample of underground uranium mines is provided in Chapter 6 . Here also is found the production cost analysis involving increased exhaust borehole vent stack height. 


\subsection{INDUSTRY DESCRIPTION}

This section will develop several general issues relating to the uranium industry and then focus on a specific study of underground uranium mines. The general topics will introduce the reader to the commodity and deposit-type characteristics of uranium, then continue on through a discussion of mining processes and mine waste disposal/reclamation. Industry statistics on production coupled with a discussion on regulations will lead to an assessment on the industry's growth potential.

The specific underground uranium mine study is a vital and major part of this report. Field trips were undertaken to a total of 31 uranium mines in five states to gather important data on population distribution, land status and 7 and value. This data was needed by the Environmental Protection Agency for the development of proposed new radionuclide emission standards for underground uranium mines. A detailed discussion is given on the methodology of study mine selection, data gathering and the results of the study.

\subsection{COMMODITY CHARACTERISTICS}

\subsubsection{Uranium Uniqueness}

A description of the uranium mining industry should begin with an understanding of the specific characteristics of the commodity of uranium itself. Is uranium unique in comparison to other metal or fuel commodities? A review of the uses of uranium, its principle industry characteristics and comparisons of these and other characteristics to different metals and fuels will help in answering the questions on the uniqueness of uranium.

Uranium has the distinction of having a dual nature--it is both a modern metal and fuel. Only plutoniun carries that same distinction. This duality might suggest a unique nitch for uranium in the marketplace, but a closer examination of the specifics of uranium will show otherwise.

Aucoin (1982) has categorized the ancient versus modern metals and fuels (Table 3.1) and discusses the properties and uses of uranium. Uranium is the youngest metal and fuel with basically only three modern uses: 
TABLE 3.1. Ancient Versus Modern Metals and Fuels

\begin{tabular}{|c|c|c|c|}
\hline Ancient Metals & Modern Metals & Ancient Fuels & Modern Fuels \\
\hline - copper & - aluminum & - coal & - natural gas \\
\hline - gold & - bismuth & - wood & - petroleum (crude oil) \\
\hline - iron & - cobalt & & - uranium \\
\hline - lead & - col umb i um & & \\
\hline - mercury & - magnesium & & \\
\hline - silver & - manganese & & \\
\hline - $\operatorname{tin}$ & - molybdenum & & \\
\hline - $\operatorname{zinc}$ & - nickel & & \\
\hline & - tantalium & & \\
\hline & - titanium & & \\
\hline & - tungsten & & \\
\hline & - uranium & & \\
\hline
\end{tabular}

Source: Aucoin (1982)

1. fuel to power nuclear reactors

2. atomic weapons

3. specific scientific purposes such as in radiation shielding. of these three functions, fuel for nuclear reactors can be considered the major use of uranium and the basis of a domestic uranium industry. Uranium thus has basically one application in this one market--that of electric power generation.

Aside from nuclear power, let us look at the major industry characteristics of uranium. Aucoin (1982) gives a list of twelve characteristics of uranium which ne states is in no particular order of priority (Table 3.2). of these twelve characteristics, Aucoin discusses some of the outstanding ones which can be compared to other commodities such as:

- High unit value. All of the precious metals (e.g., gold, silver) have this distinction.

- High political sensitivity. This can describe all of the strategic metals (e.g., the platinum group metals) plus petroleum and gas. 
- No substitutes. Another metal with this quality is manganese in its use in steel making. Manganese serves as a scavenger in removing sulfur, phosphorous and some oxygen from a molten steel batch. Of the six major ferroalloy elements, only manganese can remove sulfur. Without manganese, good steel could not be made (Dykstra and Tatnall 1976).

- Stockpiling ease. Most of the metals have similar bulk storage characteristics.

- Supply concentration. The commodity of cobalt is equally highly concentrated in its production. In 1981, five countries produced $88 \%$ of the free world cobalt, with one country producing $59 \%$. Three countries have $69 \%$ of the free world cobalt reserves.

\section{TABLE 3.2. Characteristics of Uranium}

1. Uranium usage has a moderately high projected growth rate.

2. Uranium has a high unit value.

3. Uranium has a very high political sensitivity.

4. Uranium has only one application or use.

5. The uranium-producing industry is totally demand-driven, demand is relatively price-inelastic.

6. Uranium demand forecasts are constantly being revised.

7. Uranium is a metal produced by mining.

8. Uranium users are deeply involved in exploration and production.

9. Uranium is easy and inexpensive to transport and store.

10. Uranium resources are unevenly distributed among a relatively few countries.

11. The uranium industry is diverse and is not highly organized.

12. Uranium has not as yet exhibited predictable cyclical behavior. Source: Aucoin (1982) 
Thus, uranium has many qualities in the marketplace that cannot be considered unique.

In considering the physical characteristics of uranium, as per number 7 on the list, uranium is a metal produced by mining. Grade and size of an uranium ore deposit are the two most important factors in its mining profitability. However, this fact is not unique to uranium. Grade and size relate to all metallic mining ventures. High grade ores and large tonnage ore bodies make the ideal situation, but unfortunately nature doesn't always provide such bonanzas. An ore body is usually one or the other. For example, the Rossing uranium deposit in South west Africa is a large tonnage igneous hosted ore body whose uranium mineralization is a disseminated, very low grade ore. Similarly, copper ores of today come from very low grade porphyry systems where the grade is now less than one percent copper. Mining of such lower grade ores has been made possible due to changing economic variables including the economics of scale of these mines, and new technologies. Figure 3.1 depicts the grade of uranium ore (in $\% \mathrm{U}_{3} \mathrm{O}_{8}$ ) processed from 1966-1981. The trend has been one of steady decline. In comparison, the average grade of copper ore has been declining from about $5 \%$ in 1900 to less than $0.7 \%$ today (Aucoin 1982).

A comparison of uranium prices to uranium production costs and to prices of other commodities can also be made to test the position of uranium in terms of being unique. Figure 3.2 from Aucoin (1982) depicts this for the period 1972-1981. Indexes are used versus dollars where $1972=100$ as the base index. Prices for silver, copper, and aluminum are New York spot quotes, while uranium is the NUEXCO Exchange value for $\mathrm{U}_{3} 0_{8}$. Aucoin describes the $\mathrm{U}_{3} \mathrm{O}_{8}$ production cost curve as representing "the weighted average U.S. cost, including the effects of inflation (1abor and materials escalation), productivity increases and/or decreases at both mines and mills, environmental and safety regulations, and declining ore grades plus fluctuating mill recoveries." The GNP Implicit Price Deflator is also plotted for comparison.

Of the many points the plot depicts, an important comparison can be made between the similar behavior of uranium and silver spot prices (as opposed to a 


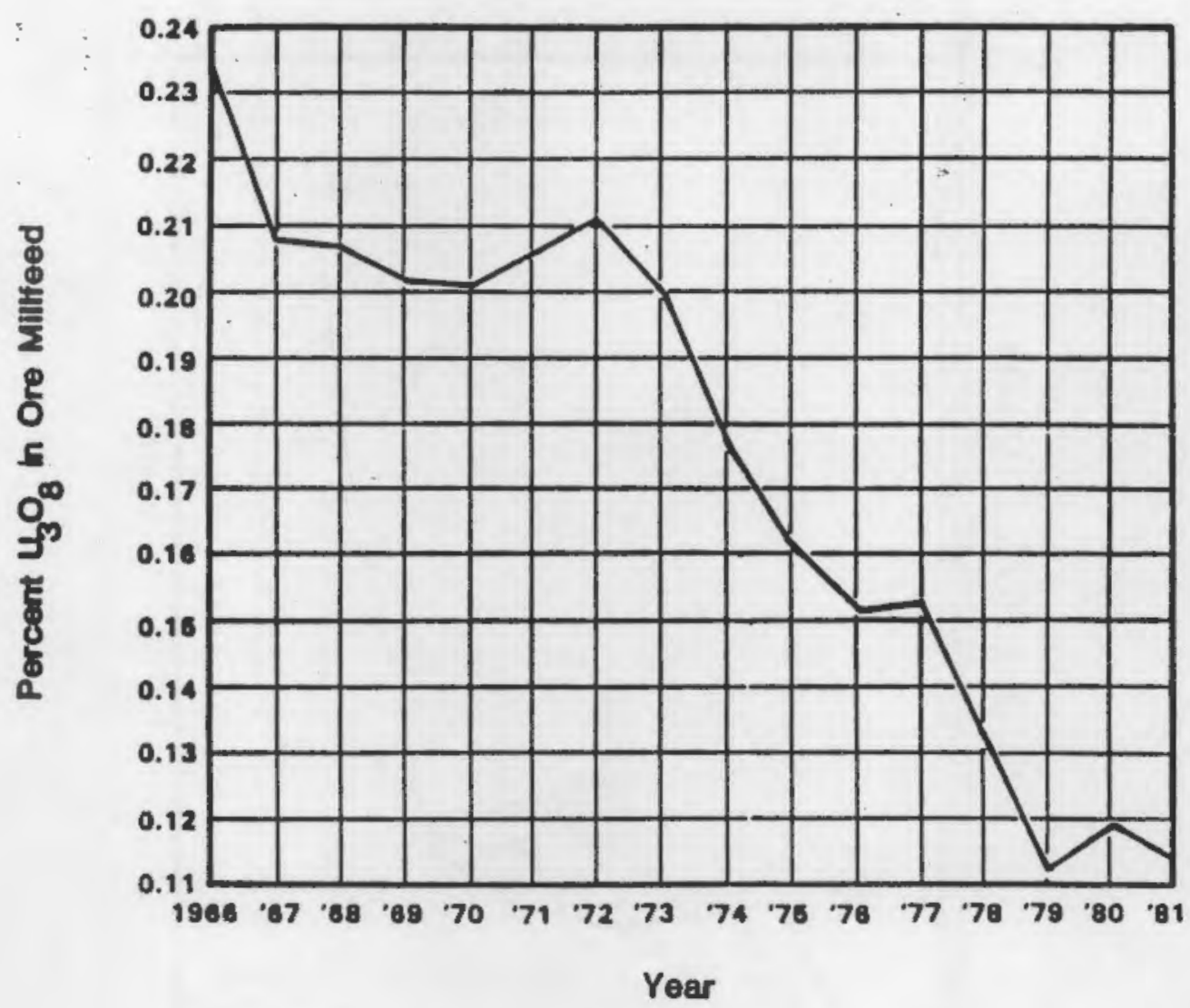

FIGURE 3.1. Grade of Uranium Ore Processed Source: Aucoin, 1982

contrast with aluminum or copper). The pricing of silver is described by Aucoin (1982) as "a tremendously complex hodge-podge of industrial demand, monetary policy, hoarders, investors, and speculators, sales from the U.S. federal stockpile, plus consumer taste in jewelry." Uranium prices can and have been influenced by these same factors i.e., speculators, hoarders, investors, industrial demand and its fluctuation, and government control and regulation. Uranium then is not unique in these terms of price behavior.

Through the review of uranium use, industry and other characteristics it has been shown that uranium is not a totally unique commodity. It may be "unique" in that it is a metal and fuel whose nearly exclusive commercial market is in power generation, but in a breakdown comparison, its many industrial and economic characteristics are similar to other commodities. The fact 


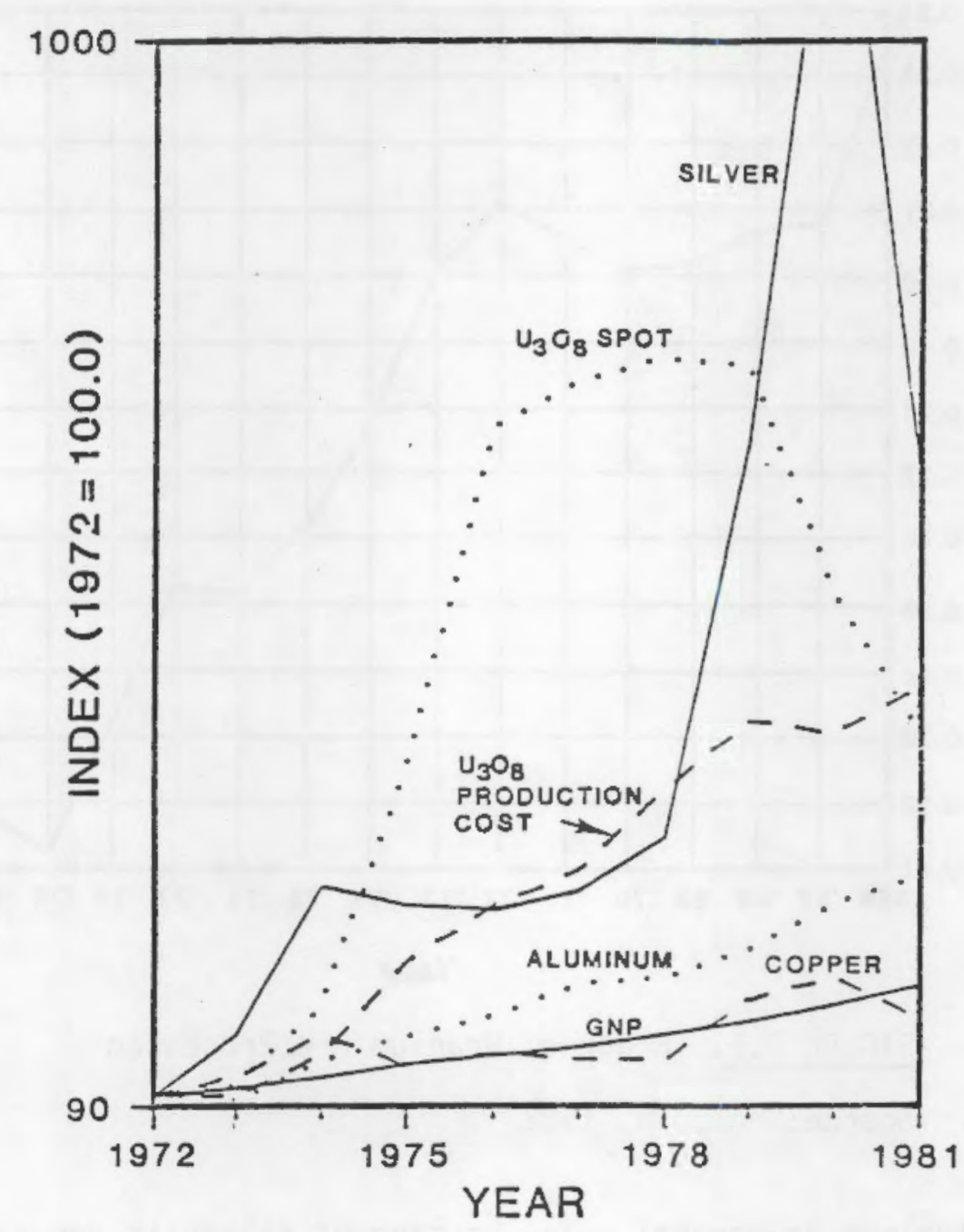

FIGURE 3.2. Uranium Prices Compared to Uranium Production Costs and to Prices of Other Commodities

Source: Aucoin, 1982

that uranium is a metal makes for its similarity in exploration, production and mining and economics among other metals. Among metals then, it is not unique at all. Uranium can only be considered unique in its specialized governmental controls and trade patterns.

\section{1 .2 Terminology}

Having an understanding of the characteristics of uranium as a commodity, a description of uranium terminology common to the commercial industry is necessary. The term "uranium" refers to several aspects of the substance such 
as: uranium the element, mineralization, reserves, ore, concentrate, yellowcake, $\mathrm{U}_{3} \mathrm{O}_{8}, \mathrm{UF}_{6}, \mathrm{UO}_{2}$, natural, enriched and depleted uranium.

In describing the chemical nature of uranium, elemental uranium refers to the atomic form. It is the neaviest of the naturally occurring elements with an atomic weight of 92 . There are two naturally occurring isotopes, ${ }^{235} U$ and ${ }^{238} \mathrm{U}$. The first one, ${ }^{235} \mathrm{U}$, is the fissile isotope, capable of splitting after the absorption of a neutron. The later, $238 \mathrm{U}$, is the fertile isotope, being convertible to the fissile plutonium. Natural uranium is characterized by the specific ratio of the two isotopes $235 \mathrm{y} / 238 \mathrm{y}$. The natural composition is about $0.71 \% 235 \mathrm{U}$ by weight (Thrush 1968). Enriched uranium has this ratio increased above its natural percentage for use as a nuclear reactor fuel (Thrush 1968). Depleted uranium is that uranium from the tails of the enrichment process with less than $0.71 \%{ }^{235} \mathrm{U}$ by weight (Thrush 1968).

In considering the physical concentration of uranium, both geologically and in processing, we refer to the terms mineralization, ore, reserves, concentrate and refined compounds. Uranium mineralization is simply its existence in certain geologic environments without reference to any specific economic parameters. Ore is that material mined containing uranium-bearing minerals with the intent to process for the extraction of its uranium content. Reserves are those ore deposits exploitable under certain economic conditions. Uranium concentrate or yellowcake is the processing (concentrating) product. It is a fine powder containing mainly three compounds of uranium (Thrush 1968):

$\begin{array}{ll}\text { ammonium diuranate } & \left(\mathrm{NH}_{4}\right)_{2} \mathrm{U}_{2} \mathrm{O}_{7} \\ \text { sodium diuranate } & \mathrm{Na}_{2} \mathrm{U}_{2} \mathrm{O}_{7} \\ \text { triuranium octoxide } & \mathrm{U}_{3} \mathrm{O}_{8}\end{array}$

Two refined compounds include $\mathrm{UF}_{6}$ and $\mathrm{UO}_{2}$. $\mathrm{UF}_{6}$, uranium hexafluoride, is the process fluid in the gaseous diffusion refining process. $\mathrm{UO}_{2}$, uranium dioxide, is a brown black powder in crystalline or pellet form used to pack nuclear-fuel rods (Thrush 1968).

\subsubsection{Chemistry}

Describing the chemical aspects of uranium as discussed in Merritt (1971), finds that the average crustal concentration to be 2-4 parts per milition (ppm). 
This is comparable to the concentrations of other elements such as molybdenum, tungsten, arsenic and beryllium. Uranium averages $6 \mathrm{ppm}$ in those igneous rocks higher in their silica content, while igneous rocks lower in silica content and higher in magnesium, aluminum and iron contain less than one ppm uranium. There are some varieties of igneous rocks in the Colorado Front Range that are high in their alkali, and low in their calcium content that have up to 100 ppm uranium.

Merritt (1971) says that uranium oxidizes readily, and in that state is quite soluble, thus having the potential for being highly distributed in nature via solutions. The uranyl ion is soluble in solutions of both acidic or carbonate-bicarbonate character and will form stable complexes with the ions of sulfate and carbonate under high temperatures and pressures. These conditions are often found in the hydrothermal solutions that accompany magmatic intrusions.

Uranium in solution may be mobilized by circulating ground waters throughout rock formations. Special physical characteristics of these rock formations along with special chemical conditions can cause the uranium to become concentrated. The concentration of uranium by precipitation from the solutions may be caused by many factors including a lowering of the temperature and pressure, reducing conditions, ion exchange, neutralization, chemical replacement and so forth. Of these factors, Merritt says that the forming of uranium minerals is caused most importantly by the reduction of the soluble $\mathrm{JO}_{2}{ }^{++}$ion from the hydrothermal and ground water solutions.

Uranium can occur in a variety of host-materials as the 1 ist from Merritt (1971) shows (Table 3.3). The concept of "cyclical migration" is important in explaining uranium's presence in many of these environments. Basically, this involves redissolving, redistributing and reprecipitation of the uranium into new environments. Later metamorphism may alter again the nature of the uranium mineralizations.

A table of the more common uranium minerals is shown (Table 3.4). The great number of uranium minerals, 155 confirmed and probable species where uranium is the important elemental constituent, is due mainly to the polyvalence of uranium and its relatively high solubility of the hexavalent form. 
TABLE 3.3. Uranium Content of Selected Materials

Rock Description

High-grade veins

Vein ores

Sandstone ores

Pegmatitic ores

Uraniferous phosphates

Gold ores (South Africa)

Chattanooga Shales (U.S.)

Marine black shales
Percent $\mathrm{U}_{3} \mathrm{O}_{8}$

$0.2-1.0$

$0.05-0.4$

$0.05-0.1$

$0.005-0.03$

$0.015-0.06$

0.006

$0.001-0.02$

Source: as adapted from Merritt (1971)

\subsection{DEPOSIT-TYPE DESCRIPTIONS}

There are many "types" of uranium deposits being economically mined today. It is difficult, to classify these many ore deposits because the economic geology and scientific knowledge of uranium is not yet perfectiy understood. And, as this knowledge of uranium and the origins of these ore deposits increases, classification schemes presently in place are continually subject to revision.

However, in light of these difficulties, many classification schemes for uranium deposits are currently being used. These schemes are based on descriptive, lithologic or temporal means of organization. For convenience, the descriptive method will be used here in outlining the basic uranium deposit 


\section{TABLE 3.4. Most Common Uranium Minerals}

\begin{tabular}{|c|c|c|}
\hline Type & Name & Chemical Composition \\
\hline Oxides & Uraninite & $\left(U+{ }^{4} 1-x, u_{x}+6\right) 0_{2+x}$ \\
\hline & Pitchblende & Variety of uraninite \\
\hline $\begin{array}{l}\text { Nb-Ta-Ti complex } \\
\text { oxides }\end{array}$ & Brannerite & $(U, \mathrm{Ca}, \mathrm{Fe}, \mathrm{Th}, \mathrm{Y})(\mathrm{Tj}, \mathrm{Fe})_{2^{0}}$ \\
\hline Silicates & $\begin{array}{l}\text { Coffinite } \\
\text { Uranophane }\end{array}$ & $\begin{array}{l}\mathrm{U}\left(\mathrm{SiO}_{4}\right)_{1-\mathrm{x}}(\mathrm{OH})_{4 x} \\
\mathrm{Ca}\left(\mathrm{UO}_{2}\right)_{2}\left(\mathrm{SiO}_{3}\right)_{2}(\mathrm{OH})_{2} 5 \mathrm{H}_{2} \mathrm{O}\end{array}$ \\
\hline Phosphates & $\begin{array}{l}\text { Autunite } \\
\text { Torbernite }\end{array}$ & $\begin{array}{l}\mathrm{Ca}\left(\mathrm{UO}_{2}\right)_{2}\left(\mathrm{PO}_{4}\right)_{2} \quad 10-12 \mathrm{H}_{2} \mathrm{O} \\
\mathrm{Cu}\left(\mathrm{UO}_{2}\right)_{2}\left(\mathrm{PO}_{4}\right)_{2} \quad 12 \mathrm{HO}_{2} \mathrm{O}\end{array}$ \\
\hline Vanadates & $\begin{array}{l}\text { Carnotite } \\
\text { Tyuyamunite }\end{array}$ & 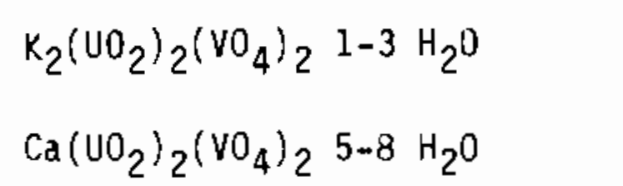 \\
\hline
\end{tabular}

Source: as adapted from Merritt, 1971

types. The descriptive method uses terms such as "sandstone type" which convey various descriptive geological characteristics. This method is also useful in that revisions need not be made to the classification scheme as increased knowledge changes the theories on origins of these deposits. 
There are ten general uranium deposit types as outlined by Cheney and Istas (1980):

- Witwatersrand type

- Blind River type

- Sandstone type

- Rossing type

- Pegmatitic type

- Surficial Uranium Deposit type

- Athabasca type

- Jabiluka type

- Veins in two mica granites types

- Other vein types

of these ten, the Sandstone type is the most important for consideration here. About $95 \%$ of the uranium produced in the United States comes from two important subdivisions of the sandstone type uranium deposit - the Wyoming subtype and Colorado Plateau subtypes (Cheney and Istas 1980).

These two deposit sub-types occur in continental arkosic sandstones derived from granitic rocks of older Precambrian age. The uranium ore usually occurs when a conglomerate or sandy facies (e.g. former stream channel) grades into a finer grained siltstone or mudstone. Mineralized horizons are commonly overlain by felsic volcaniclastic rock units. Uranium mineralization usually consists of uraninite and coffinite which act as cementing agents in the sandstone. Pyrite is also present. These minerals are commonly found in or next to coalified plant materials (Cheney and Istas 1980).

A common form of a uranium ore deposit is called the "roll" deposit. Figure 3.3 is a schematic cross-section of a typical Wyoming type uranium roll deposit. As the figure shows, the sandstone strata hosting the uranium ore is generally bounded by impermeable siltstones. These ore bodies have reached cross-sectional widths up to 25 meters in the Gas Hill district in Wyoming (Cheney and Istas 1980).

The next section will discuss the mining processes involved in extracting ore from these roll-type and other uranium deposits. 


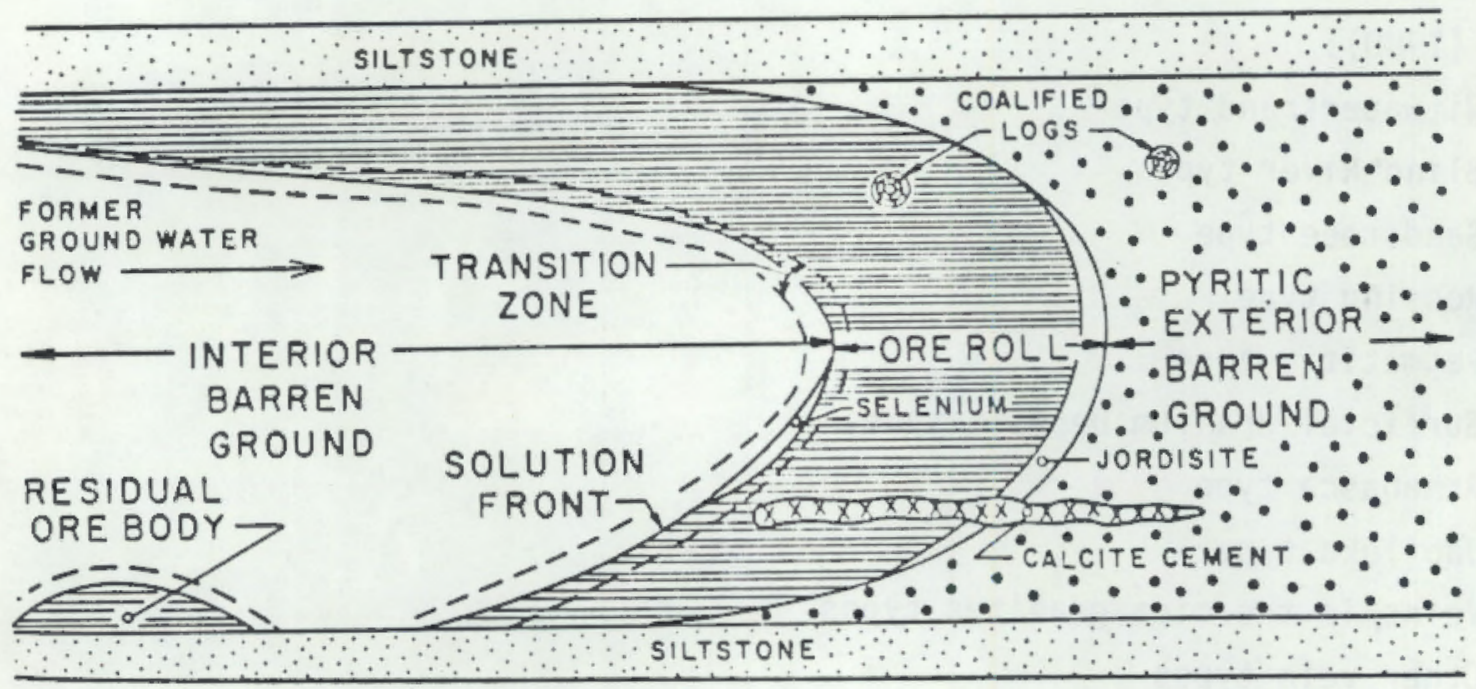

FIGURE 3.3. Schematic Cross-Section of a Wyoming-Type Roll Uranium Deposit

Source! Cheney and Istas, 1980

\subsection{MINING PROCESSES (a)}

Conventional mining techniques are utilized in the United States to extract most uranium-bearing ore. Uranium is primarily mined using underground and open-pit methods. A third form of extraction which is much less traditional is referred to as solution mining.

The choice of mining method is based on mechanical and spatial characteristics of the deposit. As a rule of thumb, open-pit mining is generally considered to be uneconomical beyond a depth of $300 \mathrm{feet}$. In the past, underground mining was favored for the Colorado Plateau regions because of the greater depths of ore occurrence. Wyoming was the site of most surface mining because of the shallow deposits and poorly consolidated overburden. Solution mining is used to recover low-grade ores that may not be economically recoverable using other methods.

(a) A glossary of mining terms is included in Appendix A to assist the reader in understanding the mining processes. 
Descriptions of uranium mining processes are provided in the following sections. Underground mining techniques are discussed first since they are the emphasis of this report. Descriptions of open-pit and solution mining methods are provided for comparison.

\subsubsection{Underground Mining}

The basic phases in underground mining include exploration, development, production, and closure. In the life of a mine, however, phase operations are of ten carried out concurrently.

\section{Exploration}

Because uranium tends to occur in irregular deposits, extensive exploration work is necessary to delineate the ore (Charles River Associates, Inc. 1977). Usually the first phase of searching for ore is the reconnaissance survey. Any geologic features or geophysical measurements that might indicate a mineral deposit are further investigated using trenching and sampling techniques. In deeper lying deposits, extensive drilling is required to appraise the deposit (Stout 1980).

\section{Development}

For most underground mines extensive preproduction development is required before production can begin. Principal steps in the development of an ore body includes 1) development drilling and 2) primary mine development.

Development drifling delineates the ore body further and assesses ore grade and amenability to ore processing. Potential locations for shafts are determined and a plan is established for mining a deposit. Information gathered during this period is required to decide whether the mine can be economically developed (Charles River Associates, Inc. 1977).

Primary mine development includes sinking of main shafts, construction of haulage ways, slopes and ventilation shafts, installation of underground equipment, and construction of surface facilities and access roads. A ventilation system is also constructed.

The first step of primary mine development is the sinking of concretelined mine shafts using mechanical shaft muckers. Most underground mines use 
vertical shaft entry except those constructed during the early war period. Lower cost adits and inclined shafts can provide entry to deposits in areas of high relief. Shafts are generally located at the end and in the center of mine property (Charles River Associates, Inc. 1977).

Shafts allow horizontal access to ore-bearing veins. From the shafts, cross-cuts are driven through the width of the ore deposit. From the crosscuts, two drifts are driven along the length of the vein. One drift undermines the ore bed and the other directly follows the bed. Each drift system comprises a horizon. When the distances between horizons is considerable, subdrifts are driven to decrease the size of blocks to be extracted.

A ventilation system must also be incorporated. Ventilation raises are constructed between the cross-cuts, drifts, subdrifts, and horizons. While working a horizon, the ore drift of the overlying level is the ventilation drift for exhausting the contaminated air. When mining a horizon, only the last ventilation connection is used for feeding fresh air to the miners. All other connections are stopped.

After the shafts and tunnels are constructed, the ore body is further delineated by longhole drilling. Exploratory boreholes in the hanging and footwalls of the deposit are used to determine the presence of mineralization in the entire width of the bed (Bykouskii, et a1. 1977).

Production

Extraction (called stoping) of an ore body begins once development is complete. The general sequence in extracting uranium ore is to drill out a blasting round, load the explosives and blast, bardown any loose material, install rock bolts for back support, excavate broken rock, and slush or tram the ore to the nearest stope exit (Dames and Moore 1980). Ore is hoisted to the surface, stockpiled and biended to the proper grade, and transported to the mill (Heeb, et al. 1980).

There are several methods of stoping employed, but room and pillar or modifications thereof are generally used. Room and pillar and modified room and pillar are methods of cutting up a deposit by excavating a grid of rooms 
separated by pillars of uniform cross-section (Battelle Columbus Laboratories 1976). Many grid lay-outs are used including both rib and square pillars with checkerboard spacing. As pillars are extracted upon retreat, the mined-out area caves and it is abandoned (Stout 1980).

Closure

When ore depletion causes a project to become unprofitable, mine closure procedures are put into effect. Such closing procedures are presently being used at the Kerr-McGee Puerco mine as described in the company's environmental report. Valuable underground equipment is salvaged and a concrete plug is poured at the collar of the production shaft to seal the mine. The area of the ore stockpile is cleaned and all structures removed. The disturbed areas are relaid with topsoil and reseeded (New Mexico Energy and Mineral Department 1979).

\subsubsection{Open-Pit Mining}

The basic phases in open-pit mining are the same as for underground mining. The exploration phase described in Section 3.3.1 is identical since mining methods are not chosen until after this phase is completed. The development phase consists of removing overburden and development drilling. Ore is removed during the production phase followed by closure and reclamation. Reclamation procedures are discussed in Section 3.4.4.

The first step in open-pit mining is the removal of overburden with scrapers or power shovels. Blasting and ripping is usually required to loosen the topsoil. When the orebody is exposed, it is cleaned of waste with tractor scrapers and bulldozers. After blasting and ripping of the ore, the ore is mined with a backhoe and loaded onto trucks for hauling to the mill.

Mining starts before the whole ore body is exposed by dividing the mine into areas. Overburden from the first mined area is stripped and placed on the surface. Overburden from succeeding areas is used to backfill areas where mining has been completed. At the cessation of operations, the overburden is covered with topsoil and reseeded (Battelle Columbus Laboratories 1976). 


\subsubsection{Solution Mining}

A third form of uranium extraction is referred to as solution mining. The two principa? forms, in-situ and heap leaching, can be used to recover uranium from low-grade ores. In-situ means leaching the ore in the geological formation in which it occurs. In heap leaching, a leach solution is applied to heaps of ore on drain tiles (Charles River Associates, Inc. 1977).

Currently in-situ mining projects for uranium use a sodium or ammonium carbonate solution for leaching of the ore (Battelle Columbus Laboratories 1976). The leach solution is injected through several outer wells and recovered in a center well. In order to contain the leachate, the ore zone should have impermeable material underlying and overlying the ore-bearing zone (New Mexico Energy and Mineral Department 1979). Recovered solutions carry from 50 to 150 ppm $\mathrm{U}_{3} \mathrm{O}_{8}$ (Dames and Moore 1980).

Heap leaching requires weeks or even years and chemical reagents such as sulfuric, nitric, or hydrochloric acid are generally used domestically. Bacterial leaching is utilized in the Elliot Lake region of Canada (Charles River Associates, Inc. 1977).

\subsection{WASTE MANAGEMENT}

Wastes generated during mining can be categorized as airborne, liquid, and solid. Wastes are generated at all phases of a mining project. Most wastes are expelled to the environment untreated and generally cause only short-term environmental impacts. Ore stockpiles, mine water-evaporation ponds, and mined-out pits must be reclaimed at the cessation of operations. The following sections discuss the waste-generating processes for all phases of a mining project. Finally, surface reclamation techniques are discussed since it is the major waste-management procedure required in a mining project.

\subsubsection{Airborne Wastes}

Various air pollutants are generated during the life of a mine. Effluents generated as a result of the operation of machinery, the construction of roads, and the drilling, blasting, blending, and loading of ore and are released to the atmosphere untreated. Airborne effluents consist of dust, combustion gases, radon gas and radon daughters. 
Effluents generated from these processes can be minimized using standard techniques. Excavated rock, loaded mine cars and trucks, and roadways can be wetted down to reduce dust emanation (Heeb, et a?. 1980). Speed of trave) should be lessened on unpaved roads (Battelle Columbus Laboratories 1976). Engine gases can be catalytically decomposed and, in some cases, atomized sprays are used to remove part of the dust from the air after loading and blasting (Heeb, et al. 1980). Although most of the effluents from these processes are minimized rather than treated, the impacts of these effluents on air quality is slight, local, and short-term (Battelle Columbus Laboratories 1976).

The ventilation-air exhaust boreholes in underground mines are another significant source of airborne wastes. Ventilation holes exhaust dust, radon, and radon daughters from the mine workings. Dilution with the atmosphere is currently used to control the concentration of contaminants in the environment (Heeb, et a1. 1980). Also, limited use of fabric or fiber filtration units clean primary air inside the mine for use in remote areas (Floyd $C$. Bossard and Associates 1983).

Personnel at Battelle Columbus Laboratories recently overviewed radon control measures in underground mines. Currently no process or device is being used to reduce radon at the shaft, but several possible methods have been proposed. Processes include electrostatic precipitation and filtration, gas centrifuging, cryogenics, and chemical removal methods. Stacks installed at exhaust points would also assist in dilution.

Control methods that reduce mine levels of contaminants, also tend to reduce atmospheric discharge of wastes. Methods inc1ude bulkheading of closedoff areas and the establishment of appropriate pressure differentials in the sealed-off area so that contaminants do not escape. Water is used to suppress dust in various areas of the mine. Backfilling of mined-out areas not only provides ground support, but also reduces ventilation requirements. Sealants and diffusion barriers applied to mine walls (intake airways, shops, lunchrooms, and mined-out areas) can also significantly reduce radon contamination levels. 


\subsubsection{Liquid Wastes}

Mine water is the main liquid effluent leaving an underground or open-pit mining facility. Mine water is produced by the flow of both surface runoff and subsurface water into pits or mine workings. In underground mines, sump pumps are used to dewater workings. Inceptor wells located at mine perimeters can also aid in the dewatering process of underground workings (Thomsen 1983). Ground water from underground mines is pumped to nolding ponds on the mine surface. In surface mines, the water flows by gravity to a holding pond in the mine pit where it is allowed to evaporate or seep into the ground.

Water from underground mines is usually treated before it is discharged to the environment. Mine water is pumped to a holding pond on the surface and treated using ion exchange methods to remove uranium. Water from the ion exchange circuit is then treated with barium chloride and discharged to a second holding pond. Radium precipitates out and the water evaporates. At the cessation of operations, the tailings are covered and reclaimed (Heeb, et al. 1980).

In some cases, mine water is discharged to the environment without treatment. The effluent can transform dry washes and ephemeral streams to perennial streams disrupting local topography and biota. Local surface waters can be contaminated, and seepage may contaminate aquifers (Battelle Columbus Laboratories 1976).

Groundwater contamination from underground operations also occurs after mining ceases when sump dewatering has stopped. The inactive mine fills with water until the original water level is reestablished. The water is contaminated through contact with mine rubble. To reduce contamination, a number of mines in New Mexico recover uranium from flooded mines through ion exchange. Barren liquid is returned until original conditions are restored in the aquifer (Thomsen 1983).

Effluents from solution mining are not produced until the project is completed. Leach solution is recycled until the end of operation. Upon completion of the project, the solutions are evaporated in tanks or lined ponds. The dry residue is packaged and shipped to active mill tailings disposal sites (U.S. Nuclear Regulatory Comnission 1980). 


\subsubsection{Solid Wastes}

Solid wastes from undergound mining are treated using standard reclamation practices. Wastes consist of rocks and overburden excavated from pits, shafts, haulage drifts, and ventilation boreholes. There is also residue from inine water holding and treatment and dried-out ore stockpiles (Heeb, et al. 1980). Standard reclamation practices include covering with topsoil or spoils and stabilizing with vegetation or riprap.

\subsubsection{Reclamation}

The objective of reclamation is to reconstitute the disturbed area in such a manner that when operations cease, the land is suitable for alternative uses. Because they disturb much greater areas, surface mines are of much greater concern than underground or solution mines. Some reclamation is required in all cases, however.

The main objective in reclaiming mine spoils is not to reduce the escape of radioactivity as it is in milling. With mine spoils, structural stability must be achieved in order for vegetation to becone established. Spoils are usually in piles and must be graded and terraced to minimize erosion and retain rainfall.

Spoils are then covered with topsoil or treated with chemicals if vegetation is required. Riprap can also be used to minimize erosion. The following paragraphs outline the general procedures for stabilizing disturbed sites.

Revegetation

Vegetation is a naturally occurring protectant against erosion for any land surface. But in the case of uranium mines, establishing suitable plant species will require careful planning due to the arid climates of the sites. The revegetation procedure includes site preparation, soil treatment, seeding, and irrigation.

Site preparation involves grading and topsoil placement. A proper topsoil is selected and transported to the site by haul trucks or bulldozers. At the site, bulldozers are used to backfill and rough grade the spoils to blend with the surrounding topography; some operations use draglines or scrapers. In all cases, slopes should not exceed $3: 1$ according to personnel at Century West Engineering, Bend, Oregon. Motor patrols, bulidozers, or tractor-pulled drags 
or floats are commonly used to fine grade the spoils. Fine grading readies the spoil surface for topsoil placement by removing low places and small humps and ridges. Surface rocks are also removed by handpicking or front-end loaders. After a suitable spoils surface is obtained, the topsoil is uniformiy placed on the fine-graded spoils, usually with scrapers (Persse 1977).

Revegetation should follow topsoiling as soon as possible to prevent erosion according to personnel at Century West Engineering, Bend, Oregon. Soil preparation includes both physical (discing, plowing, etc.) and chemical (fertilizers, mulch) methods. Harrowing discs scarify the soil to allow better penetration of fertilizers and plant seeds. To enrich the soil, nitrogen or phosphorous-based fertilizers may be added in dry form using a broadcast spreader. If soil acidity must be corrected, liming agents are added with spinner-type spreaders mounted on trucks. In many cases discing must follow lime application. If lime rates exceed 10 to $20 \mathrm{mt} / \mathrm{ha}$, split applications with parts being plowed under is recommended (Mays and Bengtson 1978).

The most common methods of planting seed are drilling, broadcasting, and hydroseeding. Grass and legumes should be drilled using a drill seeder equipped with depth bands and cultipacker wheels. The seeder-cultipacker wheels are aligned to cut furrows. The drill benind the wheels puts seeds in the furrows while another set of wheels, offset from the drill, cover and compact the soil in one operation. Drill rows are ordinarily placed 20 to $40 \mathrm{~cm}$ apart at a depth of 6 to $20 \mathrm{~mm}$.

Broadcast seeding is generally used for small, inaccessible areas or areas that are seeded iminediately after grading. After roughening the area by harrowing or discing, seeds are applied with a fan or airblast seeder. An aircraft can sometimes be used for broadcast seeding if soil surfaces are rough enough for wind and water action to cover the seed. A cultipacker, harrow or disc, or a sheeps foot roller can also be used for covering the seed.

Seeds can also be planted using a hydroseeder which mixes it with water and sprays. Hydroseeding alone has not usually produced good strains of vegetation in semiarid regions. However, good results are obtained when hydrofertilizing or mulching follows drilling or broadcast seeding. 
Trees and shrubs are sometimes planted as seeds, but this is risky. A higher degree of success is obtained from planting small plants either barerooted or potted in biodegradable containers. After hardening the plants for several weeks, tubings are usually planted by hand (Persse 1977). Since mulching improves soil filtration and reduces evaporation, it is particularly advantageous for establishing perennial species in areas of low rainfall. Wood chips, wood fiber, manure, and sewage sludge all can be utilized as mulch for revegetation purposes. Straw, hay, and sawdust are applied with a specifically designed blower or spread by hand. Wood fiber, manure, and sewage sludge are applied in a water slurry (hydromulching). Straw is probably the most effective and widely-used mulch.

Common methods of holding straw in place are crimping, discing, or rolling into the soil; covering with a net or wire; or spraying with a chemical tackifier. Crimping is accomplished by a weighted tractor-drawn carriage which forces blunt notched discs into the soil. A specially-designed roller equipped with studs is utilized for rolling or "punching." The roller may be tractordrawn on gentle slopes. However, on steep slopes with top-of-the-slope access, a flat-bed truck equipped with a winch can be utilized to raise and lower the roller. Both crimping and rolling require soft soils to allow the teeth to penetrate. But roiling can be used on much steeper slopes than a crimper can.

Nets constructed from woven kraft paper, plastic fabric, poultry netting, concrete-reinforcing wire, and even jute can be anchored to hold straw in place. The most common mulching method, however, is the use of a tackifier. This method is useful for hard soils and steep slopes with limited access. Asphalt emulsions and wood fiber-flue mixtures are applied over the straw, or simultaneously with the straw-blowing operation. Hydromulching techniques are frequently utilized (Kay 1978).

Irrigation to start a vegetative cover is usually required in arid climates, especially for the first growing season. Once the seed has germinated and the plants have been established, irrigation schedules can be gradually cut back. By the end of the second or third growing season, the system is no longer required. 
Drip irrigation is a new technique predominately used to establish sparse tree and shrub cover in arid environments. With drip irrigation, a small volume of water (1-2 gal/hr) is delivered to each plant along with fertilizers by means of an emitter. For practicality, each emitter spacing is limited to $4 \times 4$ feet. Maintenance includes installation and care of filters and inspection and flushing of plugged emitters. Tensiometers are used to adjust irrigation schedules to weather conditions and plant requirements.

Sprinkler irrigation is a universal technique for supplying supplemental water. This method provides broad, uniform area coverage, but water requirements are more than double requirements for drip irrigation. Fertilizers can be injected into the water system, too (Bengston 1979).

Low-cost water harvesting methods can be used to enhance growth and survival of vegetation. Water collecting areas lined with paraffin or black polyethylene rubber asphalt increase runoff and concentrate moisture around seedlings.

Long-term maintenance of the reclaimed area includes fertilization with nitrogen if necessary, inspection, and possible reseeding of areas that fail to thrive.

Riprap

Riprap can also be used to stabilize the spoils at uranium mine sites, especially in arid climates or areas where surface runoff is high. Riprap is basically a rock material which varies in size from small cobbles to large boulders weighing several tons. The different grades and sizes of riprap are defined in Table 3.5 .

Riprap is usually procured from quarries. The quarry floor is drilled and blasted to obtain large chunks of rock. The rock material is loaded on haul trucks and transported to a crushing and grading facility. If the quarry floor is below the water table, pumps maintain a dry floor by circulating water to a settling pond. Settling pond water is often used for the wash plant which classifies the fine rock material. Water is not essential for riprap manufacture, however. 
TABLE 3.5. Quarried Stone for Erosion and Sediment Control (a)

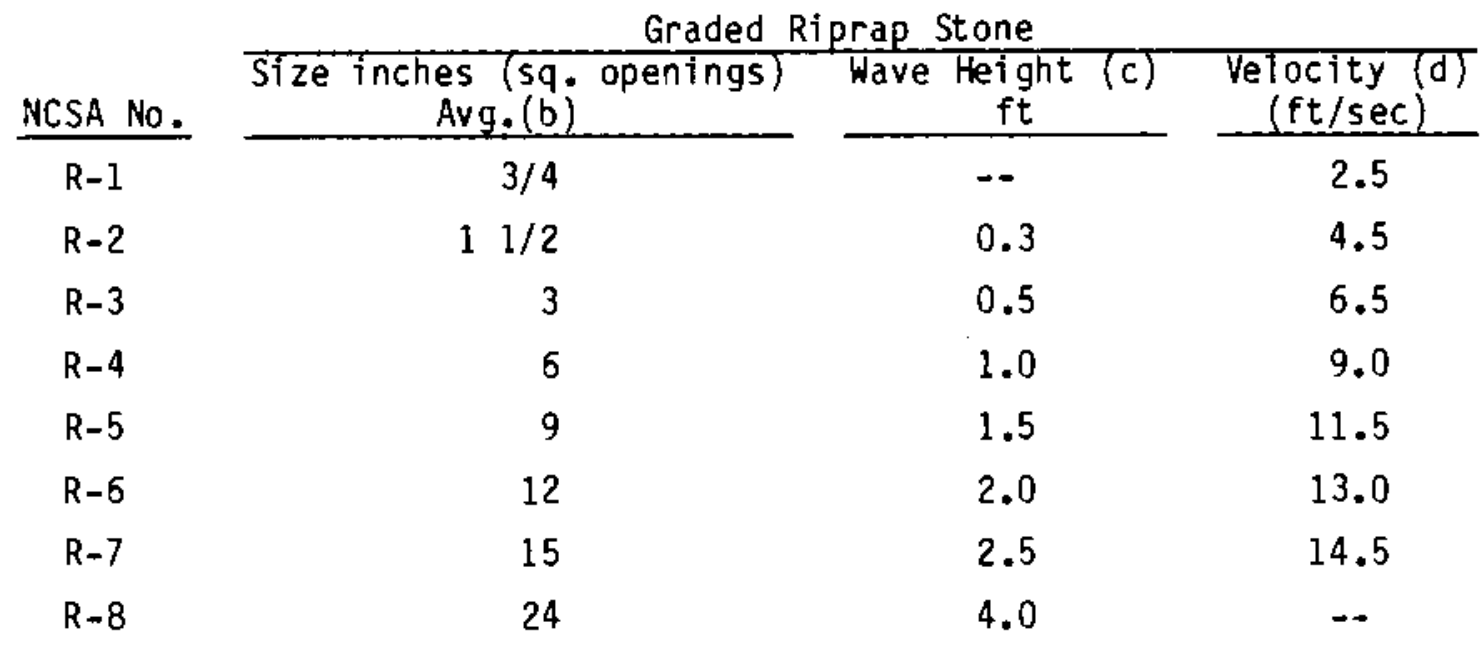

(a) The table assumes a stone dry density of 165 pounds per cubic foot.

(b) "Average size" is that size exceeded by at least $50 \%$ of the total weight of the tonnage shipped; i.e., $50 \%$ of the tonnage shall consist of pieces larger than the "average" size (normally $1 / 2$ the specified nominal top size).

(c) Wave Height is the vertical distance from wave crest to wave trough. The wave height given in the table is the average height of the 1.3 highest waves in the incident wave train.

(d) The stream velocity is the velocity at mid-stream or at a point 10 feet from the bank, whichever is closest to the bank. Source: as adapted from National Crushed Stone Association, 1978.

From the quarry the large stone pieces are transported by haul trucks to a separation facility located nearby. A typical facility would contain a vibrating grizzly, screens, and feeders. As the rock material moves along the grizzly, progressively larger stones drop through openings onto vibrating feeders. The vibrating feeders transport the rock material to screens for successive grading. Graded riprap drops to the ground. Front end loaders and haut trucks transport the riprap to stockpiles. The riprap is then loaded onto haul trucks or rail cars and transported to the mine site (Robertson 1979).

Other sources of riprap include river beds and naturally occurring gravel deposits. The processing procedure would be very similar; however, drilling and blasting would not be required. The riprap would be loaded onto front-end 
loaders. If graded riprap is desired, portable crushing and classifying equipment located nearby would then manufacture the tecnnically acceptable riprap. Pitrun riprap is simply excavated and hauled to the site.

Management requirements for riprap covers includes: 1) periodic inspections, 2) repair of damaged areas, and 3) removal of unwanted vegetation.

As with revegetation, some preparation is needed before the riprap cover can be placed on the uranium mill tailings. First, the site must be graded and whenever possible, the slope should not exceed $3: 1$. In the case of large riprap, sand-like bedding material is placed on the top of the covered tailings. The sandy material stabilizes the large stones and insures the long-term integrity of the riprap cover. Small cobblestone riprap would probably not require bedding material, however.

When the site is prepared, riprap is dumped onto the spoils. bulldozers or, in some cases, laborers spread the riprap to meet predetermined design requirements.

\subsection{MINING INDUSTRY STATISTICS}

Uranium ore is extracted by various mining methods, but as described previously, primarily from underground and open-pit mines. As reported by the Department of Energy (1983), there were 139 underground and 24 open-pit uranium mines reported in operation in 1982 accounting for a combined 75\% of uranium production. Solution and "other" mining methods accounted for about $25 \%$ of production in 1982 (Table 3.6).

The 1982 distribution of uranium ore production by state is given in Table 3.7. Production continues to be primarily from the western United States, mainiy New Mexico, Wyoming, and Texas. The amount of uranium produced from all underground uranium mines and received at mills and buying stations in 1982 equaled $2,809,000$ tons ore or 6,200 tons $U_{3} 0_{8}$. This underground ore production represented about $34 \%$ of the 1982 total receipts of $8,313,000$ tons ore (Table 3.8). 
TABLE 3.6. Distribution of 1982 Uranium Production by Mining Method(a)

Source

Underground Mines
Open-Pit Mines
Solution Mining
By-Product
Others: Heap Leach, Mine Water,
and Low-Grade Stockpiles

Totals

Number of Thousand Percent of Sources Tons $\mathrm{U}_{3} \mathrm{O}_{\mathrm{B}} \quad$ Total Production

$\begin{array}{rrr}139 & 6.3 & 46 \\ 24 & 3.8 & 29 \\ 18 & 1.5 & 11 \\ 5 & 1.6 & 12 \\ 10 & 0.2 & 2 \\ 196 & 13.4 & 100\end{array}$

(a) Table based on information from 182 operating and 14 unmined sources. Source: U.S. DOE, 1983.

TABLE 3.7. Distribution of 1982 Uranium Production by State(a)

State

New Mexico
Wyoming
Texas
Others: Arizona, Colorado, Florida,
Idaho, Utah, and Washington
Totals

Thousand Percent of Tons $\mathrm{U}_{3} \mathrm{O}_{8} \quad$ Total Production

$\begin{array}{ll}3.8 & 28 \\ 2.7 & 20 \\ 2.2 & 17\end{array}$

$4.7 \quad 35$

$13.4 \quad 100$

(a) Table based on information from 182 operating and 14 unmined sources. Source: U.S. DOE, 1983.

In other terms, the DOE (1983) reported that total U.S. production of uranium concentrate equalled 14,430 tons $U_{3} 0_{8}$. Of this total, 10,115 tons came from ore, while 3,315 tons came from other sources. This total concentrate production is down $30 \%$ (or 5810 tons) from the 1981 level of 19,240 tons (Table 3.9).

Uranium drilling declined as well in 1982. Uranium companies drilled only 6.1 million feet in 1982, down 8 million feet or 56\% from the 1981 level of 14.1 million feet (Table 3.10). 

TABLE 3.8. Uranium Ore Produced from Mines and Received
at Mills and Buying Stations

\begin{tabular}{|c|c|c|c|c|}
\hline$a n$ & $\begin{array}{cr}\text { Total Receipts } \\
\text { Ore } & \text { U308 }\end{array}$ & $\begin{array}{c}\text { (Thousand Tons) } \\
\text { Open-Pit Production } \\
\text { Ore U308 }\end{array}$ & $\begin{array}{c}\text { Underground } \\
\text { Ore }\end{array}$ & $\begin{array}{l}\text { Production } \\
\text { U308 }\end{array}$ \\
\hline 1982 & 8,313 & 5,504 & 2,809 & 6.2 \\
\hline
\end{tabular}

(a) This represents mined ore only; does not include production from mine water, in-situ leach liquor, heap-leach solutions, by-products, and miscellaneous low-grade ore from old mine dumps.

Source: U.S. O0E, 1983.

TABLE 3.9. Ore Processed and Concentrate Produced in 1982

\begin{tabular}{|c|c|c|c|c|c|}
\hline \multirow[b]{2}{*}{ State } & \multicolumn{2}{|c|}{ Ore Processed } & \multicolumn{3}{|c|}{$\begin{array}{c}\text { Concentrate Produced } \\
\left.\text { (Tons } \mathrm{U}_{3} \mathrm{O}_{8}\right)\end{array}$} \\
\hline & $\begin{array}{l}\text { Millions } \\
\text { of Tons }\end{array}$ & Tons $\mathrm{U}_{3} \mathrm{O}_{8}$ & From Ore & Other $(a)$ & Total \\
\hline $\begin{array}{l}\text { New Mexico } \\
\text { Wyoming } \\
\text { Arizona, Colorado, Florida, }\end{array}$ & $\begin{array}{l}2.11 \\
3.89\end{array}$ & $\begin{array}{l}3,755 \\
2,550\end{array}$ & $\begin{array}{l}3,650 \\
2,440\end{array}$ & $\begin{array}{r}255 \\
80\end{array}$ & $\begin{array}{l}3,905 \\
2,520\end{array}$ \\
\hline $\begin{array}{l}\text { Louisiana, Texas, Utah, } \\
\text { and Washington }\end{array}$ & 2.75 & 4,215 & 4,025 & 2,980 & 7,005 \\
\hline Totals & 8.75 & 10,520 & 10,115 & 3,315 & 13,430 \\
\hline
\end{tabular}

(a) Includes $\mathrm{U}_{3} \mathrm{O}_{8}$ in concentrate obtained by solution mining, heap leaching, and as a by-product from other activities.

Source: U.S. DOE 1983.

One uranium industry statistic remained the same in 1982 as it was in 1981. The average grade of ore processed was $0.12 \% \mathrm{U}_{3} \mathrm{O}_{8}$. However, the recovery of $\mathrm{U}_{3} \mathrm{O}_{8}$ from ore processed was $96 \%$ up $2 \%$ from the year before (U.S. DOE 1983). 
TABLE 3.10. Comparison of 1982 and 1981 Orilling by State

\begin{tabular}{|c|c|c|}
\hline State & $\begin{array}{l}1982 \text { Orilling } \\
\text { (Millions of Feet) } \\
\end{array}$ & $\begin{array}{l}1981 \text { Drilling } \\
\text { (Millions of Feet) }\end{array}$ \\
\hline $\begin{array}{l}\text { Wyoming } \\
\text { Texas } \\
\text { New Mexico } \\
\text { Utah } \\
\text { Colorado } \\
\text { Others }\end{array}$ & $\begin{array}{l}1.8 \\
1.2 \\
0.6 \\
0.6 \\
0.8 \\
1.1\end{array}$ & $\begin{array}{l}3.8 \\
3.9 \\
1.5 \\
1.9 \\
1.0 \\
2.0\end{array}$ \\
\hline Totals & 6.1 & 14.1 \\
\hline
\end{tabular}

(a) For 1982, includes Alaska, Arizona, Cal i fornia, Michigan, Minnesota, Montana, Nebraska, Nevada, Oklahoma, Oregon, South Dakota, Virginia, Washington, and undisclosed locations. Eighty-eight percent of this drilling was done in the states of Arizona, Nebraska, and South Dakota.

Source: IJ.S. DOE, 1983.

\subsection{INDUSTRY EMISSION REGULATIONS}

Radioactive emissions from underground uranium mines, i.e., radon daughter products found in mine atmospheres have been identified as a health risk to miners and individuals situated near mining operations (MGinley, 1975). McGinley (1975), discusses that these radioactive emissions have been the assumed cause of lung cancer at a higher than expected incidence rate among underground uranium miners. These emissions discharged to the above-ground atmosphere are also thought to be a health concern for individuals living proximal to such operations. There has been an evolution of standards governing radioactive exposure/emissions. These standards deal with two aspects: 1) the exposure to the underground uranium miner, 2) and the emission 1 imit to the above ground atmosphere.

\subsubsection{Underground Exposure}

MCGinley (1975) states that around 1960, a systematic monitoring of radondaughter concentrations in underground uranium mines was implemented by state agencies and major mining companies and standards were developed. The United States Public Health Service (USPHS) in 1959, the American Standards 
Association (ASA) (now known as the American National Standards Institute (ANSI)) and the United States Atomic Energy Commission (AEC) in 1960 recommended that an exposure standard be set for underground uranium mines based on a maximum permissible radon-daughter concentration of one working level (WL) in the mine atmosphere.(a) This standard stated that when any working area showed a working level between 1.0 and 3.0 , a determination was to be made of the weighted average exposure to workers. If a greater than $10.0 \mathrm{WL}$ was measured, immediate action was necessary to reduce the exposure to workers and correct conditions. The average exposure level for the industry was $7 \mathrm{WL}$ in 1957 with a drop to $2.1 \mathrm{WL}$ by 1966 (McGinley 1975).

The Federal Radiation Council (FRC) was established in 1959 (Public Law 82-373) to provide guidance for federal agencies in regards to formulating radiation standards and enforcing programs in conjunction with the states involved in such mining. The FRC published in September 1967, a recommendation of no more than six working level months (WLM) exposure to a miner over a 3 month consecutive period and no more than 12 WLM in any 12 month consecutive period. It was recommended also that actual exposures were to be kept lower than this standard when at all practical and that records of exposure to individuals were to be kept.

A prior standard proposed by the Department of Labor earlier in the same year (May, 1967) called for a much lower 0.3 WLM. After much debate and study, a final standard as recommended by the FRC was adopted limiting exposure to 4 WLM as of July 1, 1971 (MCGinley, 1975). It is listed in the Code of Federal Regulations (CFR) under 30 CFR 57 - "Safety and Health Standards - Metal and Non-Metal Underground Mines." This standard has remained unchanged as of May, 1983 as indicated in the Code of Federal Regulations, List of CFR Sections Affected, May, 1983.

As described by Breslin (1981), there are three federal agencies that are involved in Mine Health and Safety and in overseeing this exposure standard. They are the Bureau of Mines (BOM) the Mine Safety and Health Administration

(a) A working level is defined as any combination of radon-daughters ip one liter of air that will result in the ultinate emission of $1.3 \times 10^{5} \mathrm{MeV}$ of potential alpha energy (McGinley, 1975). 
(MSHA) and the National Institute for Occupational Safety and Health (NIOSH). The BOM is responsible for research and development on mine safety and control. of the mine environment. MSHA is responsible for enforcement and promulgation of mine health and safety standards. NIOSH is responsible for research on the health of miners and the recommendation of health standards.

\subsubsection{Emission to Atmosphere}

With the establishment of the Environmental Protection Agency (EPA), the FRC was abolished and its responsibilities transferred to the EPA. The EPA on December 27,1979 , listed radionuclides as a hazardous air pollutant under provisions of the Clean Air Act, Section 112. Congress in 1977, had amended this act to consider such air borne emissions of radioactive materials and directed the EPA to determine whether emissions of radioactive pollutants cause or contribute to air pollution. After listing radionuclide emissions as hazardous, the EPA began efforts for their regulation in coordination with the Nuclear Regulatory Commission (NRC) and the Department of Energy (DOE). Prior to these provisions in the Clean Air Act of 1977, these emissions were mostly unregulated or regulated under the earlier Atomic Energy Act, (a) (Federal Register, 1983).

The Sierra Club filed suit against the EPA in California on June 16 , 1981. The club alleged that it was the nondiscretionary duty of the EPA to propose standards for radionuclides within 180 days after listing them as hazardous pollutants. The suit resulted in a court order on September 30 , 1982. It stated that the EPA must publish proposed regulations, with a notice of hearings, within 180 days of the order (Nuclear News, 1983a).

(a) Under authority of the Atomic Energy Act of 1954, as amended; Reorganization Plan No. 3 of 1970 and as listed in the Federal Register, Vol. 42, p. 4860, January 13, 1977, there are environmental radiation protection standards for nuclear power operations. These standards apply to radiation doses received by public members in the general environment resulting from operations which are part of a nuclear fuel cycle. Nuclear fuel cycle here excludes mining operations by definition. However, this standard limits the annual dose equivalent not to exceed 25 millirems to the whole body, effective December 1, 1979 (40 CFR 190). 
The EPA has identified four types of facilities as sources of radionuclide emissions, which includes underground uranium mines. They have proposed standards for the emissions from these sources. The standard for underground uranium mines proposes to restrict the increase in annual average concentration of ${ }^{222} \mathrm{Rn}$ in any unrestricted area around a mine. The restriction is 0.2 picocuries per liter ( $\rho \mathrm{Ci} / 2$ ) (Federal Register, 1983). An unrestricted area means any area not under the control of the mining company or government agency (Federal Register, 1983).

It is noted in the Federal Register (1983) that the EPA has concluded that there is presently no practical control technology to effectively reduce ${ }^{222} \mathrm{Rn}$ emissions to air from underground uranium mines. However, the 1977 amendments to the Clean Air Act allow the EPA to set design, equipment, work practice, or operational standards for such hazardous materials as radionuclides when a emission standard may not be achievable (White, et al., 1979).

Thus, the EPA has suggested various methods for meeting the proposed emission standard. As listed in the Federal Register (1983), they are:

1. Reducing the operating time of the mine, 2. Increasing the stacks effective heights, 3.Controlling additional land around the mine vents - out to $2 \mathrm{~km}$. The least expensive method for meeting the proposed standard is expected to be controlling land within $2 \mathrm{~km}$ (Federal Register, 1983).

\subsection{INDUSTRY GROWTH POTENTIAL}

Presented in Table 3.11 are the number of reactors that began commercial operation in each year from 1974 through 1982. After 1977, the rate of reactor startups has dropped significantly. In 1978, the last reactor was ordered and since 1972 more than one hundred reactors have been canceled. In 1982 , "14 reactors were delayed (had their construction schedules stretched out); 18 more representing 22019 megawatts capacity--were canceled" (Raloff 1983). 
TABLE 3.11. Number of Reactors Starting Commercial Operation

$\begin{array}{lc}\frac{\text { Year }}{1974} & \text { Number of Reactors } \\ & 13 \\ 1975 & 8 \\ 1977 & 3 \\ 1978 & 8 \\ 1979 & 2 \\ 1980 & 2 \\ 1981 & 2 \\ 1982 & 4 \\ & 2\end{array}$

Source: Nuclear News, February $1983 d$.

As stated previously, of the three main uses of uranium, fuel for nuclear reactors can be considered the major use of uranium and the basis for a domestic uranium industry. The continued existence of this uranium industry thus is directly dependent on the existence of a domestic nuclear power industry. The present nuclear power industry faces many problems that threaten its own expansion. In summing up the major problems, Cart Walske, president of the Atomic Industrial Forum, has been quoted by Science News magazine (Raloff 1983) as saying "that the nuclear power industry's top ... problems are money ..., regulation, public acceptance and waste disposal." Similarly, the U.S. Department of Energy, Energy Information Administration (1983) has summarized its views on the conditions necessary in the nuclear power industry for a longterm resurgence in new nuclear power orders to be possible. These include:

- The need for new electric generating capacity clearly increases.

- Nuclear power remains competitive with alternative generation sources, such as coal.

- Utility financial practices and utility rate structures are modified to reduce debt equity and cash-flow burdens of new nuclear construction. 
- Uncertainties surrounding nuclear generating plants are resolved, including the predictability of the nuclear licensing process, nuclear safety regulations, reactor siting, and long-term uranium availability.

- The nuclear waste disposal problem is resolved, particularly the construction of a Federal repository for the long-term disposition of highly radioactive wastes.

Given below are a few examples and details of these problems/conditions in the nuclear power industry as quoted from recent literature.

- In a report released January 21, 1983 by the National Economic Research Associates, Inc. (NERA) entitled The Current Economics of Electric Generation from Coal in the U.S. and Western Europe, "NERA has concluded that nuclear has an advantage of 15 to $25 \%$ over coal east of the Mississippi and west of the Rockies, but adds that the uncertainties surrounding future nuclear costs are so great that it will likely tend to make utilities favor coal-fired units for new orders" (Nuclear News, 1983b).

- Continuing on this topic, Lewis J. Perl, senior vice president of NERA, states that "nuclear's decline is due to several factors ... largely economics in origin. Both capital and operating costs have escalated rapidly in the past five years. If this continues, nuclear's small advantage over coal will completely evaporate" (Catalano 1983b).

- The lifetime of nuclear reactors has typically been estimated to be about 30 years and some plants have already operated 20 years. There is now great concern that the average life of all reactors will not be 30 years. In the same report cited above, NERA states that "the nuclear advantage would be eliminated if the lifetime of nuclear plants turns out to be shorter than has been previously anticipated (20 instead of 30 years)." (Nuclear News, 1983b) 
- According to Lee Catalano, Industry News Editor for Power Magazine, "capacity factors [of operating commercial reactors] have not been as high as expected. At $60 \%$, they are about $10 \%$ under the expert's predictions." (Catalano 1983a). Economically, this tends to reduce nuclear power's cost advantage over other competing electric sources such as coal-fired plants.

- The average lead time for construction of a reactor in the U.S. has "historically been eight years although in contrast, the anticipated average lead time for reactors now under construction is 10 to 12 years" (Bonny and Fulton 1982). Utilities borrow to finance construction, and with higher interest rates and longer lead times construction costs have also increased.

- On the waste disposal issue, in April of this year a "unanimous U.S. Supreme Court ... rul[ed] that California and other states may ban future plants until the federal government creates permanent disposal sites for radioactive wastes ... Al ready seven states besides California have passed moratorium legislation" (Newsweek, May 2, 1983)

Not only do all the problems that affect the nuclear power industry affect the uranium industry, but the domestic uranium industry has problems unique unto itself.

- Paul C. DeVergie and others of the U.S. Department of Energy in the Grand Junction Area Office state that:

"In terms of constant 1981 dollars, the current price is close to the all-time low value which occurred in 1972 . Yet, the real cost of uranium production continues to rise, engendered to a considerable degree by increasingly stringent environmental regulations. Substantial portions of the investments made to develop the large uranium deposits discovered in the 1970s have resulted in little or no actual production. Additional expenditures 
will be needed to maintain or reopen completed or partially completed mine development workings if they are to be used in the future. As a result of these conditions, uranium production, investments, exploration activities, and industry employment have dropped very sharply during the past 2 years and are projected to decline even further in the future" (DeVergie et al. 1982).

- Lastly, "U.S. producers ... are adjusting to the industry's reduced requirements. More reactors have been canceled here, and production costs are the highest anywhere. ... Foreign producers have stepped into the U.S. market. Presently, about $28 \%$ of all uranium in the U.S. is imported, and this figure is expected to increase" (Catalano 1983a). Thus, domestic producers are losing market share in a declining market that is characterized by large and increasing inventories.

\subsection{UNDERGROUND URANIUM MINE STUDY}

A group of 29 underground uranium mines was chosen for study in regards to population, land status and land value surrounding their sites. This group represents approximately $84 \%$ of the 1982 domestic underground uranium production. (a) The methodology used in selecting this representative group of 29 underground uranium mines, plus the procedure used in estimating its percent of industry production will be discussed. Also, a description of uranium miner productivity and estimates of mine-specific production, plus a detailed characterization of population, land status and land value will be presented in the following sections.

\subsubsection{Study Mine Selection and Background}

The 29 mines in this study were selected based on mine type, operating status and cumulative employee-hours as reported by the U.S. Department of Labor-Mine Safety and Health Administration (MSHA) (1982) listing. The MSHA

(a) Calculated using 3rd quarter, 1982, statistics from the Department of Labor-Mine Safety and Health Administration (MSHA). 
list was first broken down according to mine type and operating status. Only those underground mines (code 01-"type") that were listed as active (code 01 "status"), or known to be recently inactive, were considered. For this group, 105 mines total, employee-hours worked were then converted to a full-time equivalent (FTE) worker number. (a)

These 105 mines were then categorized according to operating status and FTE. Operating status was either active or temporarily inactive and the FTE breakdown was greater than 25, 25-10, 9-1, and 0.9-0 FTE (Table 3.12). From this categorization, the 30 mines falling under the greater than $25 \mathrm{FTE}$ and 25-10 FTE were selected as the initial underground uranium mine study group.

TABLE 3.12. Categorization of MSHA Uranium Mines

\begin{tabular}{|c|c|c|c|c|}
\hline \multirow[b]{2}{*}{ Mine Status } & \multicolumn{4}{|c|}{ Full-Time Equivalents ${ }^{(a)}$ (FTE) } \\
\hline & $\geq 25$ & $\underline{25-10}$ & $\underline{9-1}^{(c)}$ & $0.9-0(c)$ \\
\hline $\begin{array}{l}\text { (Total number mines in each category) } \\
\text { Active } \\
\text { Temporarily inactive }\end{array}$ & ${ }_{3}^{20}(b)$ & ${ }_{2}^{5}(b)$ & $\begin{array}{r}29 \\
6\end{array}$ & $\begin{array}{l}25 \\
15\end{array}$ \\
\hline
\end{tabular}

(a) 1 FTE $=2000$ man hours.

(b) These were indicated as "active" in the MSHA list. Personal opinion at time chart was made indicated "inactive" (no production) status.

(c) It was not known that ventilation was applied full-time to all of these mines.

A later modification of the selection scheme, however, resulted in the total dropping to 29 mines. This modification combined two of the mines into one entity (Hacks Canyon $\$ 1$ and \#2), added two mines (Pigeon and Kanab North) due to their proximity and similarity to the Hacks Canyon area, and dropped one mine (Bill Smith) due to information that the mine was flooded. (b) And

(a) One FTE equals 2000 man hours.

(b) Since data on the mine was collected during a field excursion in the area, the data is included in this report, but as supplemental information only. It has not been includer into the final tabulation totals. 
finally, data for the Schwartzwalder was not included in the final tabulated totals. (a) This decision not to include the Schwartzwalder data was made by the EPA because the mine was considered not to be typical of the U.S. uranium mining industry due to its deposit type (vein-type versus sandstone-type) and location (mountain resort area near metropolitan Denver, Colorado). Without the Schwartzwalder and Bill Smith, the study group number totals 29 (Table 3.13).

Information for each of the 29 study mines is summarized on individual synops is sheets in Appendix $C$. All sources of information on these sheets are referenced. Location maps are also given in Appendix 0 , showing all occupied dwellings, mine shaft locations, and population distributions.

\section{Percentage of Industry Production}

The estimated percentage of 1982 underground uranium production represented by these 29 study mines (84\%) was calculated from the MSHA employeehours data. This data is available quarterly from the Health and Safety Analysis Center, Denver, Colorado. Even though actual production statistics are not generally publicly available on a mine-by-mine basis, estimates of a production percentage can be made using employee-hour statistics. This estimate of the percentage of industry production as represented by the 29 study mines should be representative of the actual production percentage if certain assumptions are clearly stated as follows:

1) Worker productivity is relatively constant between mining locations.

2) The MSHA data gives the best data as to the "operating status" of a particular underground uranium mine unless other higher confidence information indicates differently.

3) The MSHA employee-hours for mines of status 1, 2, and 3 (active, temporarily inactive, or permanently closed, respectively) will be used in the calculation, but under the following scheme:

(a) Since data on the mine was collected during a field excursion in the area, the data is included in this report, but as supplemental information only. It has not been included into the final tabulation totals. 
TABLE 3.13. Uranium Mines in Study Group

MINE

Arizona

Hack Canyon

Pigeon

Kanab North

Colorado

Sunday

King Solomon

Deremo-Snyder

New Mexico

Mt. Taylor

01d Church Rock

Church Rock NE

Church Rock \#1

Church Rock East

Kerr-McGee

Sec. 30 East

Kerr-McGee

Sec. 30 West

Kerr-McGee Sec. 19

Kerr-McGee Sec. 35

Kerr-McGee Sec. 36
COMPANY

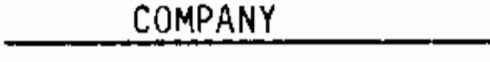

Energy Fuels

Nuclear, Inc.

Energy Fuels

Nuclear, Inc.

Energy Fuels

Nuclear, Inc.

Union Carbide Corp.

Union Carbide Corp.

Union Carbide Corp.

Gulf Mineral Resources

United Nuclear Corp.

United Nuclear Corp.

Kerr-MeGee Corp.

Kerr-McGee Corp.

Kerr-McGee Corp.

Kerr-McGee Corp.

Kerr-McGee Corp.

Kerr-McGee Corp.

Kerr-McGee Corp.
LOCATION

T37N, R5W, Sec. 26, Mohave Co., Arizona

T38N, R2W, Sec. 5, Coconino Co., Arizona

T38N, R3N, Sec. 17,

Mohave Co., Arizona

T44N, R18W, Sec. 13, San Miguel Co., Colorado

T48N, R17W, Sec. 19, Montrose Co., Colorado

T42N, R20W, Sec. 2, San Miguel Co, Colorado

T13N, R8W, Sec. 24, Cibola Co., New Mexico T16N, R16W, Sec. 17, McKinley Co., New Mexico T17N, R16W, Sec. 35, McKinley Co., New Mexico T17N, R16W, Sec. 35, Mckinley Co., New Mexico T17N, R16W, Sec. 36, McKinley Co., New Mexico T14N, R9W, Sec. 30, McKinley Co., New Mexico T14N, R9W, Sec. 30 , Mckinley Co., New Mexico T14N, R9W, Sec. 19, Mckinley Co, New Mexico T14N, R9W, Sec. 35, McKinley Co., New Mexico T14N, R9w, Sec. 35, McKinley Co., New Mexico 
TABLE 3.13. (Contd)

Homestake Sec. 23

Homestake Sec. 25

Nose Rock

Mariano Lake

Utah

velvet

Tony $M$.

(Lucky Strike)

Wilson-Silverbel1

Lisbon

LaSal

Hecla

Wyoming

Big Eagle

Golden Eagle

Sheep Mountain

Supplemental Data

Schwartzwalder (a)

Bill Smith (b)
Homestake Mining $\mathrm{Co}$.

Homestake Mining Co.

Phillips Petroleum Co.

Gulf Mineral Resources

Atlas Corp. - Minerals Division

Plateau Resources, LTD.

Innion Carbide Corp.

Rio Algom Corp.

Union Carbide Corp.

Union Carbide Corp.

Pathfinder Mines Corp.

Silver King Mines

Western Nuclear, Inc.

Cotter Corp.

Kerr-McGee Corp.
T14N, R10W, Sec. 23, McKinley Co., New Mexico

TI4N, R10W, Sec. 25, McKinley Co., New Mexico

TI9N, R11W, Sec. 31, McKinley Co., New Mexico

TI5N, RI4W, Sec. 12, McKinley Co., New Mexico

T31S, R25E, Sec. 3, San Juan Co., Utah

T35S, R11E, Sec. $16 \& 21$, Garfield Co., Itah

T32S, R26E, Sec. 15

San Juan Co., Utah

T29S, R24E, Sec. 21, San Juan Co., Utah T29S, R24E, SeC. 1, San Juan Co., Utah T29S, R24E, Sec. 6, San Juan Co., Utah

T27N, R92W, Sec. $2 \& 11$, Fremont Co., Wyoming T36N, R73W, Sec. 14, Converse Co., Wyoming T28N, R92W, Sec. 22, Fremont $\mathrm{Co}$., Wyoming

T2S, R71W, Sec. 25 Jefferson Co., Colorado T36N, R74W, Sec. 36 , Converse Co., Wyoming.

(a) Given as data supplemental only because the mine type and location are not considered typical of the uranium industry.

(b) Data given as supplemental only because of reported mine flooding. 
a. For those study mines that are known to be "not producing" or that have become "jnactive" or "closed" prior to 1982, MSHA employee-hours will not be used and considered to the zero (hence zero production).

b. For all other underground mines, employee-hours will be used for on ly those of status 1 , status 2 (becoming "inactive" sometime in 1982) and status 3 (becoming "closed" sometime in 1982).

The formula for calculating the estimated percentage of production is estimated as follows:

$$
\frac{P}{100}=\frac{\Sigma S M H}{\Sigma M H}=\frac{\sum S M P R}{\sum M P R}
$$

where

$P=$ percent of total underground uranium production represented by study mines

SMH = employee-hours for study mines

$M H=$ total employee hours for all underground mines

SMPR = uranium production for study mines

MPR = uranium production from all underground mines

Based on the MSHA data (3rd quarter, 1982), a value of 84\% of total 1982 underground production from the 29 study mines is calculated as follows:

$$
0.84=\frac{3,602,498 h r^{(a)}}{3,602,498 h r^{(a)}+219,655 h r^{(b)}+202,140 h r^{(c)}+274,055 h r^{(d)}}
$$

(a) All study mines reporting 3rd quarter hours except Pigeon, Nose Rock, Golden Eagle and Sheep Mountain mines. These four exceptions are either not yet producing or were closed prior to 1982. Refer to Table 3.14.

(b) All other underground mines with status 1 and status 2 (becoming inactive sometime in 1982). The Schwartzwalder mine is listed separately from this category since it is reported in Table 3.14 as supplemental data.

(c) All other underground mines with status 3 (becoming closed sometime in 1982). The Bill Smith mine (supplemental data Table 3.14) drops out due to closure since 1979.

(d) Swartzwalder mine, status 1, reported separately since reported in Table 3.14 as supplementa? data. 
Table 3.14 lists the 3rd quarter 1982 employee-hours used for each of the 29 study mines in this calculation.

\section{Mine-Specific Production}

As well as estimating the production percentage represented by these 29 mines, the annual productions on a mine-by-mine basis can also be be estimated using the same employee-hour data plus a worker productivity factor. It should be emphasized that an assumption is made here that worker productivity is relatively constant between mining locations. This may not necessarily be the case on a mine-specific basis due to varying worker population throughout the country.

A productivity factor of 2.44 tons $\mathrm{U}_{3} \mathrm{O}_{8}$ per FTE uranium worker per year (underground uranium mines), calculated from DOE (1983) and MSHA (U.S. Dept. of Labor 1982) data, can be used to estimate annual productions. (a) The annual production is estimated as follows:

$$
\mathrm{APS}_{\mathbf{i}}=(\mathrm{CH})_{i}(\mathrm{~F})
$$

where APS $=1982$ annual production in tons $\mathrm{U}_{3} \mathrm{O}_{8}$ for each study mine $i$ $\mathrm{CH}=$ cumulative employee-hours for 1982 for each study mine $i$ $F=$ factor of employee productivity.

Table 3.14 lists the 4th quarter, 1982, cumulative employee-hours for each study mine, the FTE worker equivalent and the estimated mine production.

\subsubsection{Study Mine Site Characterization}

The 29 mine sites of the study group were each visited during the months of January and February, 1983, by a field team from Battelle Pacific Northwest Laboratories. For each mine site, information was gathered concerning population status, land status, and value of surrounding land out to 5 kjlometers from the site. Any associated facilities of major importance and surrounding land characteristics were also noted in the field. Results are tabulated in Tables $3.15,3.16,3.17$, and 3.18, and Appendix E.

(a) Refer to Appendix B for detailed calculations. 
TA8LE 3.14. Employee-hours, FTE workers, and Estimated Production

\begin{tabular}{|c|c|c|c|c|}
\hline Mine & $\begin{array}{l}\text { 3rd Qtr., } 1982 \\
\text { Employee-hours }\end{array}$ & $\begin{array}{l}\text { 4th Qtr., 1982 } \\
\text { Employee-hours }\end{array}$ & $\begin{array}{c}\text { 4th Qtr.., } 1982 \\
\text { FTE Worker } \\
\text { Equivalent (b) }\end{array}$ & $\begin{array}{l}\text { Estimated } 1982 \\
\text { Production } \\
\text { (tons } \mathrm{U}_{3} \mathrm{O}_{8} \text { ) }\end{array}$ \\
\hline \multicolumn{5}{|l|}{ Arizona } \\
\hline Hack Canyon & 91,292 & 113,799 & 56.9 & 138.8 \\
\hline $\begin{array}{l}\left(\begin{array}{lll}A 1 & 1\end{array}\right) \\
\text { Pigeon }\end{array}$ & 8,823 & 13,948 & 7.0 & $0^{(d)}$ \\
\hline Kanab North & 0 & 0 & 0 & 0 \\
\hline \multicolumn{5}{|l|}{ Colorado } \\
\hline Sunday & 59,196 & 75,231 & 37.6 & 91.7 \\
\hline King Solomon & 53,926 & 68,544 & 34.3 & 83.7 \\
\hline Dereno-Snyder & 81,109 & 105,316 & 52.7 & 128.6 \\
\hline \multicolumn{5}{|l|}{ New Mexico } \\
\hline Mt. Taylor & 504,130 & 592,447 & 296.2 & 722.7 \\
\hline 01d Church Rock & 50,275 & 51,535 & 25.8 & 63.0 \\
\hline Church Rock NE & 288,807 & 310,020 & 155.0 & 378.2 \\
\hline Church Rock $\# 1$ & 292,236 & 318,751 & 159.4 & 388.9 \\
\hline Church Rock East & 124,404 & 130,469 & 65.2 & 159.1 \\
\hline Kerr-McGee Sec. 30 East & 176,733 & 215,665 & 107.8 & 263.0 \\
\hline Kerr-McGee Sec. 30 West & 200,394 & 238,846 & 119.4 & 291.3 \\
\hline Kerr-McGee Sec. 19 & 191,064 & 229,450 & 114.7 & 279.9 \\
\hline Kerr-McGee Sec. 35 & 295,792 & 351,838 & 175.9 & 429.2 \\
\hline Kerr-McGee Sec. 36 & 169,580 & 200,666 & 100.3 & 244.7 \\
\hline Homestake Sec, 23 & 280,687 & 376,578 & 188.3 & 459.5 \\
\hline Homestake Sec. 25 & 118,446 & 122,347 & 61.2 & 149.3 \\
\hline \multicolumn{5}{|l|}{ New Mexico } \\
\hline Nose Rock & 35,378 & 45,041 & 22.5 & $0^{(d)}$ \\
\hline Mariano Lake & 60,693 & 66,403 & 33.2 & $81.0^{(\mathrm{e})}$ \\
\hline
\end{tabular}


TABLE 3.14. (contd)

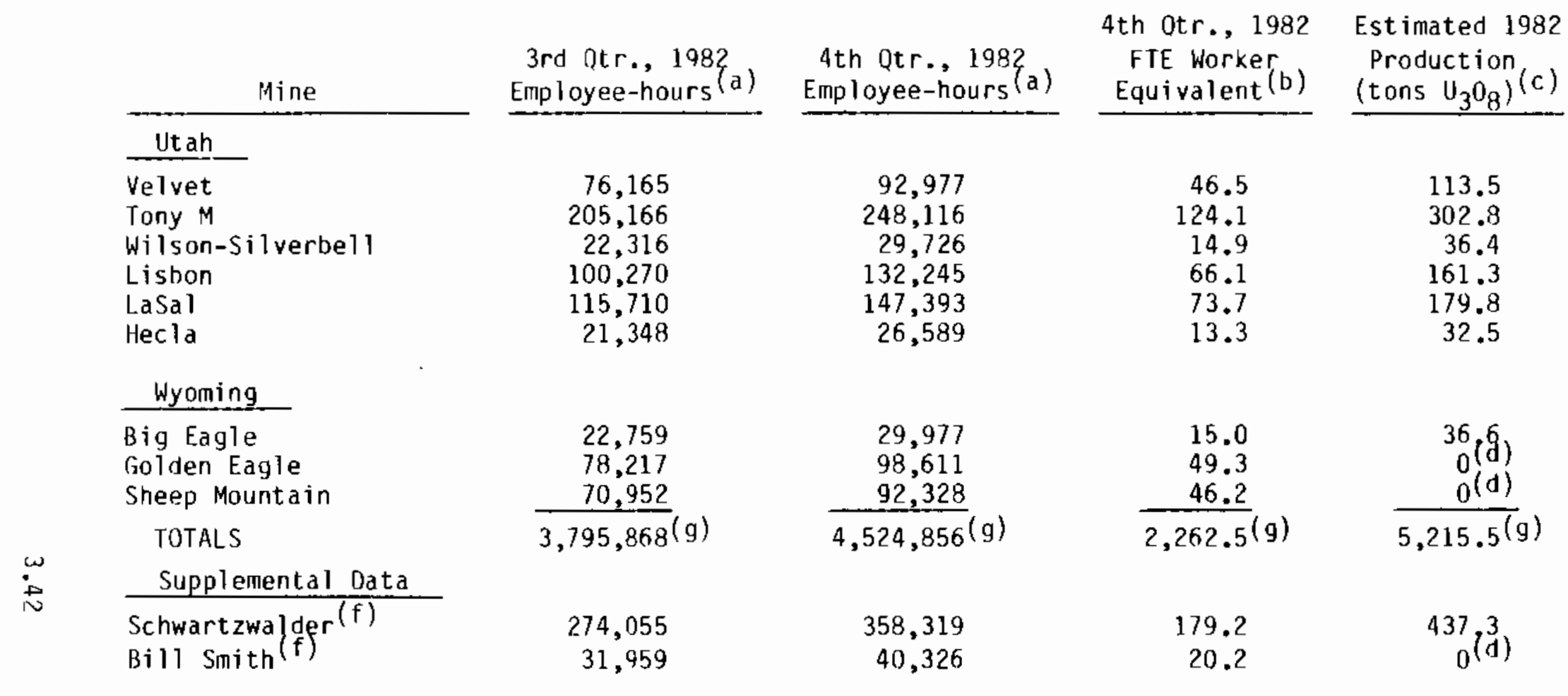

(a) Includes only those hours reported by the mining company; does not include hours reported by contractors.

(b) 1 FTE worker = cummulative employee-hours

(c) Calculated by using 2.44 tons $U_{3} 0_{8}$ per FTE worker per year. See Appendix $B$ for this productivity calculation. These are estimates only using industry average productivity and employee-hour data. Actual production may vary due to specific conditions at each mine.

(d) Zero production is reported for this mine in 1982 even though employee-hours are given. The mine is either not yet producing or is shut down prior to 1982. Consequently, the 3rd quarter company employee-hours for this mine are not included in the calculation of percentage of industry production as represented by the 29 study mines.

(e) A 1982 production figure is reported even though the mine was closed sometime in 1982 . Production occurred up until then and most likely afterwards as well, as cleanup of ore and reclamation continued throughout the remainder of the year.

(f) Hours not included in total hours of study mine group because these mines were dropped from the group for reasons cited in the text. Number of mines total 29.

(g) Represents $84 \%$ of the underground uranium industry. 
TABLE 3.15. Average Persons Per Household

(1980 Census)

\begin{tabular}{|c|c|c|}
\hline State & County & $\begin{array}{c}\text { Average } \\
\text { Persons/Household } \\
\end{array}$ \\
\hline \multirow[t]{3}{*}{ New Mexico } & Mckinley & 3.73 \\
\hline & Valencia/Cibola & 3.17 \\
\hline & & (4.35 Indian) \\
\hline \multirow[t]{2}{*}{ Utah } & San Juan & 4.04 \\
\hline & Garfield & 3.00 \\
\hline \multirow[t]{4}{*}{ Colorado } & Dolores & 2.84 \\
\hline & San Miguel & 2.55 \\
\hline & Montrose & 2.84 \\
\hline & Jefferson & 2.82 \\
\hline \multirow[t]{2}{*}{ Wyoming } & Fremont & 2.98 \\
\hline & Converse & 2.99 \\
\hline \multirow[t]{2}{*}{ Arizona } & Coconino & 3.15 \\
\hline & Mojave & 2.63 \\
\hline
\end{tabular}

Source: U.S. Department of Commerce, Bureau of Census, 1980.

\section{Population Status}

As described earlier, the EPA has suggested that one possible method of meeting the proposed radionuclide emission standard would be to restrict habitation within a zone around the mine site by controlling land out to $2 \mathrm{~km}$. Having control over surrounding land in this manner would require present residents to relocate. The EPA requested an on-site field study be conducted to determine the number of residents within a $5 \mathrm{~km}$ radius of each mine site. The latest 1980 Bureau of Census statistics were used to supplement the field study. This included population estimates for any small towns and villages located within a $5 \mathrm{~km}$ zone, and the number of people per household for each particular county and state. In addition, information was obtained from the USPHS Indian Hospital in Gallup and Crownpoint, NM. Table 3.15 is the census data incorporated into this study. 
TABLE 3.16. Population

Mine

$0-1 / 2 \mathrm{~km} \quad 0-1 \mathrm{~km} \quad 0-2 \mathrm{~km} \quad 0-3 \mathrm{~km} \quad 0-4 \mathrm{~km} \quad 0-5 \mathrm{~km}$

Arizona

Hack Canyon

Pidgeon

Kanab North

$\begin{array}{llllll}1 & 1 & 1 & 1 & 1 & 1 \\ 0 & 0 & 0 & 0 & 0 & 0 \\ 0 & 0 & 0 & 0 & 0 & 0\end{array}$

Colorado

Sunday

King Solomon

Deremo-Snyder

0
0
0

0

$\begin{array}{rrrr}0 & 0 & 0 & 0 \\ 0 & 0 & 0 & 0 \\ 21 & 49 & 67 & 83\end{array}$

New Mexico

Mt. Taylor

01d Church Rock

Church Rock NE

Chureh Rock \#1

Church Rock East

Kerr-McGee Sec 30 East

$\begin{array}{rr}0 & 0 \\ 5 & 21\end{array}$

83

Kerr-McGee Sec 30 West

Kerr-McGee Sec 19

100

317

$336 \quad 336$

336

Kerr-McGee Sec 35

Kerr-McGee Sec 36

$\begin{array}{rr}9 & 70 \\ 11 & 22 \\ 11 & 22\end{array}$

139

187

364

$11 \quad 22$

26

31

$\begin{array}{llll}22 & 27 & 31 & 31\end{array}$

31

000

Homestake Sec 23

Homestake Sec 25

Nose Rock

Mariano Lake

3

3

57

131

3

5

$$
0
$$

0

0

0

$\begin{array}{rrrrrr}0 & 0 & 0 & 0 & 26 & 35 \\ 13 & 44 & 75 & 196 & 274 & 352\end{array}$

3

70

3

$\begin{array}{lll}5 & 5 & 6 \\ 4 & 4 & 4\end{array}$

$\begin{array}{lll}0 & 0 & 0\end{array}$

$\begin{array}{lll}0 & 0 & 0\end{array}$

$\begin{array}{lll}3 & 3 & 4\end{array}$

$\begin{array}{lll}0 & 0 & 0\end{array}$

Utah

Velvet

Tony $M$

Wi Ison-Silverbell

Lisbon

LaSa 1

Hecla

13

44

196

352

Wyoming

Big Eagle

Golden Eagle

Sheep Mtn.

TOTALS

0

0

0

0

16

16

$\begin{array}{rrrrr}0 & 0 & 0 & 0 & 0 \\ 0 & 0 & 0 & 0 & 0 \\ 0 & 0 & 12 & 20 & 23 \\ 0 & 0 & 4 & 44 & 44 \\ 0 & 53 & 101 & 194 & 194 \\ 16 & 20 & 40 & 73 & 73\end{array}$

Supplemental 0ata

$$
\begin{aligned}
& \text { Schwartzwa der(a) } \\
& \text { Bill Smith }
\end{aligned}
$$

$\begin{array}{rrrrrr}0 & 0 & 0 & 0 & 0 & 0 \\ 0 & 0 & 0 & 6 & 6 & 5 \\ 0 & \frac{0}{205} & \frac{0}{618} & \frac{0}{1009} & \frac{0}{1375} & \frac{12}{1733}\end{array}$

$\begin{array}{ll}3 & 3 \\ 0 & 0\end{array}$

63

102

136

147

(a) Data given as supplemental only because the mine type and location are not considered typical of the uraniuin industry. Data gathered during a field excursion to the area.

(b) Data given as supplemental only because of reported mine flooding. Data gathered during a field excursion in the area. 
TABLE 3.17. Indian Population and Total Relocation Costs

\begin{tabular}{|c|c|c|c|c|c|c|}
\hline Site & $0-1 / 2 \mathrm{~km}$ & $0-1 \mathrm{~km}$ & $0-2 \mathrm{~km}$ & $0-3 \mathrm{~km}$ & $0-4 \mathrm{~km}$ & $0-5 \mathrm{~km}$ \\
\hline 0ld Church Rock & 9 & 9 & 70 & 139 & 187 & 364 \\
\hline Church Rock NE & 0 & 11 & 22 & 26 & 31 & 31 \\
\hline Church Rock 1 & 0 & 11 & 22 & 27 & 31 & 31 \\
\hline Church Rock East & 0 & 0 & 9 & 57 & 70 & 131 \\
\hline Kerr-McGee Sec 30 East & 0 & 0 & 0 & 0 & 0 & 1 \\
\hline Homestake $\operatorname{Sec} 23$ & 0 & 0 & 0 & 3 & 3 & 4 \\
\hline Nose Rock & 0 & 0 & 0 & 0 & 26 & 35 \\
\hline Mariano Lake & 13 & 44 & 75 & 196 & 274 & 352 \\
\hline TOTALS & 22 & 75 & 198 & 448 & 622 & 949 \\
\hline $\begin{array}{l}\text { Total Relocation } \\
\text { Costs }(\$)\end{array}$ & 396,000 & $1,350,000$ & $3,564,000$ & $8,064,000$ & $11,196,000$ & $17,082,000$ \\
\hline
\end{tabular}


TABLE 3.18. Land Ownership Percentages, $P / M / G(a)$

\begin{tabular}{|c|c|c|c|c|c|c|}
\hline Mine & $0-1 / 2 \mathrm{~km}$ & $0.1 \mathrm{~km}$ & $0-2 \mathrm{~km}$ & $0-3 \mathrm{~km}$ & $0-4 \mathrm{~km}$ & $0-5 \mathrm{~km}$ \\
\hline \multicolumn{7}{|l|}{ Arizona } \\
\hline Hack Canyon & $0 / 0 / 100$ & $0 / 0 / 100$ & $0 / 0 / 100$ & $0 / 0 / 100$ & $0 / 0 / 100$ & $0 / 0 / 100$ \\
\hline Pidgeon & $0 / 0 / 100$ & $0 / 0 / 100$ & $0 / 0 / 100$ & $0 / 0 / 100$ & $0 / 0 / 100$ & $0 / 0 / 100$ \\
\hline Kanab North & $0 / 0 / 100$ & $0 / 0 / 100$ & $0 / 0 / 100$ & $0 / 0 / 100$ & $0 / 0 / 100$ & $1 / 0 / 99$ \\
\hline \multicolumn{7}{|l|}{ Colorado } \\
\hline Sunday & $0 / 0 / 100$ & $0 / 0 / 100$ & $0 / 0 / 100$ & $3 / 1 / 97$ & $8 / 1 / 91$ & $10 / 1 / 89$ \\
\hline King Solomon & $0 / 0 / 100$ & $0 / 2 / 98$ & $0 / 5 / 95$ & $0 / 3 / 97$ & $0 / 3 / 97$ & $0 / 3 / 97$ \\
\hline Deremo-Snyder & $84 / 0 / 16$ & $87 / 0 / 13$ & $84 / 0 / 16$ & $89 / 0 / 11$ & $85 / 0 / 15$ & $81 / 0 / 19$ \\
\hline \multicolumn{7}{|l|}{ New Mexico } \\
\hline Mt. Taylor & $75 / 19 / 6$ & $58 / 26 / 16$ & $55 / 16 / 29$ & $45 / 13 / 42$ & $39 / 10 / 51$ & $39 / 7 / 54$ \\
\hline $\begin{array}{l}\text { Old Church } \\
\text { Rock }\end{array}$ & $0 / 0 / 100$ & $0 / 0 / 100$ & $0 / 0 / 100$ & $0 / 0 / 100$ & $2 / 0 / 98$ & $3 / 0 / 97$ \\
\hline $\begin{array}{l}\text { Church Rock } \\
\mathrm{NE}\end{array}$ & $0 / 0 / 100$ & $0 / 7 / 93$ & $0 / 23 / 77$ & $0 / 13 / 87$ & $0 / 8 / 92$ & $0 / 5 / 95$ \\
\hline $\begin{array}{l}\text { Church Rock } \\
\# 1\end{array}$ & $0 / 0 / 100$ & $0 / 7 / 93$ & $0 / 23 / 77$ & $0 / 13 / 87$ & $0 / 8 / 92$ & $0 / 5 / 95$ \\
\hline $\begin{array}{l}\text { Church Rock } \\
\text { East }\end{array}$ & $0 / 0 / 100$ & $0 / 7 / 93$ & $0 / 6 / 94$ & $3 / 4 / 93$ & $5 / 2 / 93$ & $3 / 1 / 96$ \\
\hline $\begin{array}{l}\text { Kerr-McGee } \\
\text { Sec } 30 \text { East }\end{array}$ & $11 / 89 / 0$ & $4 / 91 / 5$ & $2 / 70 / 28$ & $4 / 78 / 18$ & $10 / 79 / 11$ & $13 / 77 / 10$ \\
\hline $\begin{array}{l}\text { Kerr-McGee } \\
\text { Sec } 30 \text { West }\end{array}$ & $11 / 89 / 0$ & $24 / 76 / 0$ & $17 / 72 / 11$ & $16 / 69 / 15$ & $22 / 666 / 12$ & $27 / 57 / 16$ \\
\hline $\begin{array}{l}\text { Kerr-1McGee } \\
\text { Sec } 19\end{array}$ & $0 / 100 / 0$ & $23 / 77 / 0$ & $46 / 39 / 15$ & $45 / 39 / 16$ & $32 / 37 / 31$ & $29 / 38 / 33$ \\
\hline $\begin{array}{l}\text { Kerr-McGee } \\
\text { Sec } 35\end{array}$ & $0 / 100 / 0$ & $0 / 85 / 15$ & $8 / 59 / 33$ & $14 / 55 / 31$ & $10 / 57 / 33$ & $14 / 52 / 34$ \\
\hline $\begin{array}{l}\text { Kerr-McGee } \\
\text { Sec } 36\end{array}$ & $5 / 42 / 53$ & $14 / 22 / 64$ & $27 / 14 / 59$ & $36 / 8 / 56$ & $36 / 5 / 59$ & $39 / 3 / 58$ \\
\hline $\begin{array}{l}\text { Homestake } \\
\text { Sec } 23\end{array}$ & $74 / 0 / 26$ & $68 / 0 / 32$ & $61 / 6 / 33$ & $50 / 18 / 32$ & $47 / 17 / 36$ & $53 / 12 / 35$ \\
\hline $\begin{array}{l}\text { Homes take } \\
\text { Sec } 25\end{array}$ & $100 / 0 / 0$ & $85 / 0 / 15$ & $59 / 0 / 41$ & $58 / 1 / 41$ & $50 / 2 / 48$ & $43 / 10 / 47$ \\
\hline Nose Rock & $0 / 50 / 50$ & $0 / 50 / 50$ & $0 / 45 / 55$ & $0 / 41 / 59$ & $0 / 38 / 62$ & $0 / 35 / 65$ \\
\hline Mariano Lake & $0 / 0 / 100$ & $0 / 0 / 100$ & $0 / 0 / 100$ & $0 / 0 / 100$ & $0 / 0 / 100$ & $0 / 0 / 100$ \\
\hline
\end{tabular}




\section{TABLE 3.18. (contd)}

\begin{tabular}{|c|c|c|c|c|c|c|}
\hline Mine & $0-1 / 2 \mathrm{~km}$ & $0-1 \mathrm{~km}$ & $0-2 \mathrm{~km}$ & $0-3 \mathrm{~km}$ & $0-4 \mathrm{~km}$ & $0-5 \mathrm{~km}$ \\
\hline \multicolumn{7}{|l|}{ Utah } \\
\hline Velvet & $14 / 0 / 85$ & $10 / 0 / 90$ & $6 / 0 / 94$ & $12 / 0 / 88$ & $24 / 0 / 76$ & $27 / 0 / 73$ \\
\hline Tony $M$ & $0 / 0 / 100$ & $0 / 0 / 100$ & $0 / 0 / 100$ & $0 / 0 / 100$ & $0 / 0 / 100$ & $0 / 0 / 100$ \\
\hline $\begin{array}{l}\text { Wilson- } \\
\text { Silverbell }\end{array}$ & $80 / 0 / 20$ & $95 / 0 / 5$ & $95 / 0 / 5$ & $94 / 0 / 6$ & $91 / 0 / 9$ & $81 / 0 / 19$ \\
\hline Lisbon & $0 / 0 / 100$ & $0 / 0 / 100$ & $6 / 0 / 94$ & $17 / 2 / 81$ & $21 / 2 / 78$ & $16 / 1 / 83$ \\
\hline LaSal & $8 / 0 / 92$ & $25 / 0 / 75$ & $34 / 0 / 66$ & $41 / 0 / 59$ & $34 / 0 / 66$ & $26 / 0 / 74$ \\
\hline Hecla & $25 / 0 / 75$ & $25 / 0 / 75$ & $48 / 0 / 52$ & $37 / 0 / 63$ & $28 / 0 / 72$ & $21 / 0 / 79$ \\
\hline \multicolumn{7}{|l|}{ Wyoming } \\
\hline Big Eagle & $0 / 100 / 0$ & $0 / 88 / 12$ & $0 / 80 / 20$ & $0 / 8 / 92$ & $0 / 5 / 95$ & $1 / 3 / 96$ \\
\hline Golden Eagle & $60 / 20 / 20$ & $89 / 7 / 4$ & $85 / 3 / 2$ & $94 / 1 / 5$ & $91 / 1 / 8$ & $90 / 1 / 9$ \\
\hline Sheep Mtn. & $30 / 45 / 25$ & $18 / 42 / 40$ & $5 / 28 / 69$ & $2 / 18 / 80$ & $4 / 11 / 85$ & $12 / 8 / 80$ \\
\hline AVERAGES & $20 / 22 / 58$ & $22 / 20 / 58$ & $22 / 17 / 61$ & $23 / 13 / 64$ & $22 / 12 / 66$ & $22 / 11 / 67$ \\
\hline \multicolumn{7}{|c|}{ Supplemental Data } \\
\hline $\begin{array}{l}\text { Schwartzo- } \\
\text { walder }\end{array}$ & $100 / 0 / 0$ & $100 / 0 / 0$ & $100 / 0 / 0$ & $100 / 0 / 0$ & $100 / 0 / 0$ & $100 / 0 / 0$ \\
\hline Bill Smith $(c)$ & $1 / 0 / 99$ & $15 / 0 / 85$ & $34 / 0 / 66$ & $43 / 5 / 52$ & $54 / 8 / 38$ & $64 / 7 / 69$ \\
\hline
\end{tabular}

(a) $P=$ private, $M=$ mineowner, $G=$ government, federal, state, local and Indian lands

(b) Oata given as supplemental only because the mine type and location are not considered typical of the uranium industry. Data gathered during a field excursion to the area.

(c) Data given as supplemental only because of reported mine flooding. Data gathered during a field excursion in the area.

Population estimates (Table 3.16) were based on occupied dwelling counts and the noted information from the Bureau of Census USPHS Indian Hospital. Population was estimated for zones at $1 / 2,1,2,3,4,5 \mathrm{~km}$ radii from the mine site. Occupied dwelling units were identified and plotted onto the largest scale United States Geological Survey topographic quadrangle maps available or any other detailed map available such as United States Forest Service national forest district maps, United States Bureau of Mines surface-minerals management status maps, or United States Geologic Survey county series topographic maps. 
Once plotted, the total estimated number of people per zone was calculated by using the people per household statistic for the particular county and state multiplied by the number of total occupied dwellings per zone. Table 3.16 represents cumulative totals for each increasing zone distance.

In several cases, two or more mines were located within the $5-\mathrm{km}$ radius. When this occurred, the total area around the sites was allocated to the nearest mine. Refer to page 0.5 in Appendix 0 for a simple case of two intersecting mine sites (Deremo-Snyder/Wilson-Silverbell). A more complex case is shown on page 0.8 for the Ambrosia Lake Mining District (7 mine cluster).

A special note needs to be made concerning Indian population around eight of the mines in New Mexico. Refer to Table 3.17. Indian population was broken out of the totals due to special consideration regarding Indian relocation and buyout of Indian property.

Land Status

The EPA requested that the land status be identified, i.e., what percentage of the land in each zone around the mine is owned by private individuals, the mining company, or government agencies (Table 3.18). This involved reviewing public information ( $p l a t s$ and records) at respective county tax assessors' offices. In most cases, information regarding surface ownership was available in an easy-to-use format, either being plotted on a base township and range map or other reference. However, in some instances in the highly developed mining districts of western Colorado and eastern Utah, information concerning ownership of patented and unpatented mining claims was very ill-organized. Detailed examination of deed books and other submitted mining maps and documents was required in these cases. General land status information was also available from the USFS and USBM. We aggregated federal, state, local, and Indian lands in the government ownership category; these can be separated if needed.

\section{Land Valuation Methodology}

The EPA requested that private land values be determined for lands within the $5 \mathrm{~km}$ radius of each mine site (Table 3.19). Land values were determined by using several data sources: 1) information from the respective county and 
TABLE 3.19. Estimated Value of Private Land $(a), \$$

\begin{tabular}{|c|c|c|c|c|c|c|}
\hline Mine & $0-1 / 2 \mathrm{~km}$ & $0-1 \mathrm{~km}$ & $0-2 \mathrm{~km}$ & $0-3 \mathrm{~km}$ & $0-4 \mathrm{~km}$ & $0-5 \mathrm{~km}$ \\
\hline \multicolumn{7}{|l|}{ Arizona } \\
\hline Hack Canyon & $N_{A}(b)$ & NA & NA & NA & NA & NA \\
\hline Pidgeon & NA & NA & NA & NA & NA & NA \\
\hline Kanab North & NA & NA & NA & NA & NA & $\begin{array}{l}\simeq 100 \text { acres } \\
\text { of patented } \\
\text { mining claims }\end{array}$ \\
\hline \multicolumn{7}{|l|}{ Colorado } \\
\hline Sunday & NA & NA & NA & 48,000 & 208,000 & 384,000 \\
\hline King Solomon & NA & NA & NA & NA & NA & NA \\
\hline Deremo-Snyder & 79,700 & 260,370 & 922,640 & $1,852,070$ & $3,028,940$ & $4,432,770$ \\
\hline \multicolumn{7}{|l|}{ New Mexico } \\
\hline Mt. Taylor & 39,600 & 391,500 & $2,523,700$ & $2,834,200$ & $3,227,400$ & $3,918,800$ \\
\hline 01d Church Rock & NA & NA & NA & NA & 543,300 & $1,443,100$ \\
\hline Church Rock NE & NA & NA & NA & NA & NA & NA \\
\hline Church Rock \#1 & NA & NA & NA & NA & NA & NA \\
\hline Church Rock East & NA & NA & NA & 122,200 & 355,600 & 355,600 \\
\hline Kerr-McGee Sec 30 East & 35,000 & 35,000 & 35,000 & 53,500 & 147,560 & 239,960 \\
\hline Kerr-McGee Sec 30 West & 31,100 & 132,200 & 147,800 & 157,900 & 194,800 & 235,100 \\
\hline Kerr-McGee Sec 19 & NA & 194,400 & 844,800 & $1,229,400$ & $1,405,100$ & $1,532,800$ \\
\hline Kerr-McGee Sec 35 & NA & NA & 37,000 & 137,800 & 168,000 & 336,000 \\
\hline Kerr-McGee Sec 36 & 3,400 & 23,500 & 124,300 & 336,000 & 588,000 & 977,800 \\
\hline Homestake Sec 23 & 217,800 & 528,000 & 994,100 & $1,158,700$ & $1,485,200$ & $2,361,800$ \\
\hline Homestake Sec 25 & 295,600 & 622,200 & 987,800 & $1,478,000$ & $1,632,200$ & $1,645,600$ \\
\hline
\end{tabular}


TA8LE 3.19. (Contd)

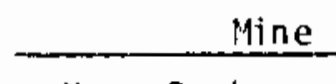

Nose Rock

Mariano Lake

Utah

velvet

Tony $M$

Wilson-Silverbell

Lisbon

LaSa I

Hecla

Wyoming

Big Eagle

Golden Eagle

Sheep Mtn.

TOTALS

Supplemental Data Schwartzwalder (c) Bill Simith $(d)$
$0-1 / 2 \mathrm{~km}$

NA

NA

$$
5,500
$$

NA

$$
39,090
$$

NA

$$
4,000
$$

36,820

$$
\begin{gathered}
\text { NA } \\
35,400 \\
18,000 \\
\hline 841,010
\end{gathered}
$$$$
880,000
$$

600
$0-1 \mathrm{~km}$

NA

NA

16,000

NA

186,350

NA

228,430

147,260

NA

$$
\begin{array}{r}
209,000 \\
42,300 \\
\hline 3,016,510
\end{array}
$$

796,200

$\frac{42,300}{9,378,270}$

$$
\begin{array}{r}
3,400,000 \\
35,330
\end{array}
$$

NA

36,000

NA

535,750

50,000

920,880

380,000

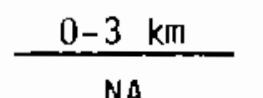

$0-4$

NA

172,800

NA

$1,667,150$

306,000

$1,427,800$

691,000

NA

$2,121,000$

$\frac{42,300}{15,835,820}$

NA

NA

$\begin{array}{r}3,584,000 \\ 150,000 \\ \hline 24,443,810\end{array}$

$5,231,000$

$\frac{898,000}{33,354,680}$

$33,600,000$

$58,400,000$

$89,200,000$

901,710

$2,008,640$

$5,151,430$

(a) Includes structures on private land only.

(b) $N A=$ not assessed. All land owned by either the mine owner or public.

(c) Data given as supplemental only because the mine type and location are not considered typical of the uraniuil industry. Data gathered during a field excursion to the area.

(d) Data given as supplemental only because of reported mine flooding. Data gathered during a field excursion in the area. 
state tax assessors' offices, 2) detailed assessed valuations from these offices and applying applicable assessment valuation to selling price ratios obtained from the U.S. Department of Commerce, Bureau of Census (1978b), 3) estimates from local real estate agents, and 4) local newspapers. The valuations were based on surface usage and rights only, since the mineral values would remain intact.

In each state and county visited, full market values were sought for private lands and dwellings. Unfortunately, each state's assessment procedures and regulations vary. Thus, to simplify matters and to offer uniformity in calculations, aggregate assessment/sales price ratios were used. Table 3.20 lists these ratios for the five states visited. As a verification measure, local real estate agencies and newspapers were checked in each state to get an approximate value of the local ranch and residential properties. Table 3.21 tists these local real estate and newspaper estimates.

Land value calculations were then made for each parcel using two basic methods: 1) using full assessment data and applying appropriate ratios or 2) using quoted local land values and multiplying by the number of acres and/or dwelling units. After determining values for methods 1 and 2 , the higher value was selected for use in this study. Citing the Mt. Taylor mine near the village of San Mateo in Cibola County, New Mexico, as an example, will clarify the calculation process.

\begin{tabular}{|c|c|c|}
\hline State & $\begin{array}{c}\text { Acreage } \\
\text { (ratio in } \%) \\
\end{array}$ & $\begin{array}{c}\text { Residential Property } \\
\text { (ratio in } 6)\end{array}$ \\
\hline Arizona & not available & 12.0 \\
\hline Colorado & 11.9 & 18.5 \\
\hline New Mexico & 9.0 & 18.5 \\
\hline Utah & 10.3 & 13.0 \\
\hline Wyoming & 5.8 & 10.1 \\
\hline Source: & $\begin{array}{l}\text { Department } 0 \\
\text { ne Census, } 19\end{array}$ & ommerce, Bure \\
\hline
\end{tabular}


TABLE 3.21. Local Real Estate Estimates for Grazing and Residential Property

$\frac{\text { State }}{\text { New Mexico }} \frac{\text { Acreage Grazing }}{\$ 240 / \text { acre }} \frac{\begin{array}{l}\text { Residential Property, } \\ \text { No Dwelling }\end{array}}{\begin{array}{l}\$ 2100 / \text { acre (San } \\ \text { Mateo area, Cibola } \\ \text { county, New Mexico } \\ \text { only) }\end{array}}$

NA $(a)$

NA

tain property

$\$ 300$ /acre flat

valley property

Utah

NA
$\$ 250$ /acre for mount-

tain property

$\$ 300 /$ acre flat

valley property
Residential Property, Occupied Owelling

$\$ 30,000 /$ residential unit; $\$ 32,700 /$ church or school unit (San Mateo area, Cibola county, New Mexico only)

$\$ 50,000 /$ residence unit

used assessed value and assessment valuation to selling price ratio
used assessed value and assessment valua- tion to selling price ratio

(a) Not applicable

The Mt. Taylor mine lies within $1 \mathrm{~km}$ of the village of San Mateo which has an estimated population of 311. The mining company operating the Mt. Taylor mine has purchased much of the private land surrounding its mining activities, owning about $7 \%$ of all property within $5 \mathrm{~km}$ of its shaft. Of the remaining property, about $39 \%$ is privately owned.

After determining the percentage of land ownership, full assessment data from the county tax assessor's office was reviewed for the $39 \%$ privately owned land. The number of acres out to $5 \mathrm{~km}$ for each of the following was determined: 1) acres of grazing land and 2) acres of residential property. In addition, the total number of occupied residences, churches and schools was determined. On a sector by sector, radius by radius accounting schene, values were determined for each property by using the full assessment and ratio method. Then, values were calculated for these same properties by using the real estate/newspaper data method. In this example, higher values resulted from the later method. Note that in these particular calculations, residential 
property is broken out into that property with and without an occupied dwelling. In the Mt. Taylor mine area, the real estate/newspaper data method values were higher than the assessment and ratio method values in all cases.

Indian Relocation Costs. As with the special breakout of Indian population (Table 3.17 ), a special consideration was made regarding land control costs of Indians. Since property is part of Indian reservations and/or Indian allotted lands, an outright buyout may not be feasible or possible. Rather a relocation of the Indian people along with a purchase of existing dwellings and new housing/property acquisition is considered. Table 3.17 gives the costs of relocating the Indian population. Such a relocation scheme is presently underway by the Federal government, involving the Hopi and Navajo tribes in the southwestern United States. Information supplied by the Navajo and Hopi Relocation Commission in Flagstaff Arizona (1981) indicates an average Indian relocation cost of about $\$ 18,000$ per person. Details of this data are as follows $(a)$ :

$\$ 71,000$ average for site and housing cost

This average figure includes a $\$ 66,000$ maximum allowance for new housing for a family of four or more, or \$50,000 maximum for a family of 3 or less, a site preparation cost encompassing $\$ 750$ for a tribal archeological survey, $\$ 1,500$ $\$ 2,000$ for sewage, and $\$ 2,000$ for a water cistern. No administration costs are reflected in this average site-housing figure.

\section{$\$ 5,000$ bonus per head-of-household}

This bonus is being offered to the Hopi and Navajo Indians as an incentive to move.

$\$ 500$ moving expense per head-of-household

This is a one time cash payment for moving expense. the client can choose between the cash or a reimbursement of actual moving expenses. In all cases to date, the $\$ 500$ has been taken.

(a) Information from Buck McGee, Chief Housing Acquisition and Relocation officer, Navajo and Hope Relocation Commission, Flagstaff, Arizona. 
0 to $\$ 1,000$ additional search/processing expenses per head of household This variable expense covers traveling expenses incurred during the search and processing for the relocation.

The average persons per household on the Hopi/Navajo reservation is 4.35. From this data, an estimate of the per capita relocation cost can be made as follows:

$$
\begin{array}{ll}
\$ 16,500 & \text { site and housing } \\
\$ 1,200 & \text { bonus incentive } \\
\$ \quad 300 & \text { moving and search expenses } \\
\$ 18,000 & \text { total relocation expense per capita. }
\end{array}
$$

Estimated Cost of Controlling Land. The estimated annual cost of controlling the land out to $2 \mathrm{~km}$ for ail mines, except the Schwartzwalder and Bill Smith is $\$ 3,012,000$ (Table 3.22). This estimate is based on the following as sumptions:

$\begin{array}{ll}\text { Fraction of total value in land } & .80 \\ \text { Fraction of total value in structures } & .20 \\ \text { Interest rate applied to land value } & .10 \\ \text { Fixed charge rate applied to depreciable } & .30 \\ \quad \text { costs based on } 5 \text { years life } & \$ 5,000 \\ \text { Per capita relocation expenses (non-Indian) } & \$ 18,000\end{array}$

TABLE 3.22. Annualized Cost of Controlling Land to $2 \mathrm{~km}$

$\begin{array}{lrr} & \frac{\text { Total Cost }}{\text { Total private land value }} & \text { Annualized Cost } \\ \text { Land }(80 \%) & \$ 9,378,270 & \\ \text { Structures }(20 \%) & 7,502,616 & \$ 750,000 \\ \text { Relocation expenses } & 1,875,654 & 563,000 \\ \text { Non-Indian (420 people) } & 2,100,000 & 630,000 \\ \text { Indian (198 people) } & 3,564,000 & \frac{1,069,000}{\$ 3,012,000}\end{array}$


Increase in $\mathrm{U}_{3} \mathrm{O}_{8}$ Production costs. The estimated increase in $\mathrm{U}_{3} \mathrm{O}_{8}$ costs to control land out to $2 \mathrm{~km}$ is $\$ 0.29$ per pound $\mathrm{U}_{3} \mathrm{O}_{8}$. This is obtained by dividing the $\$ 3,012,000$ annual cost by the estimated yearly production from mines in our population study $(10,416,000)$. The mines in our study are estinated to produce $84 \%$ of the 1982 production of $\mathrm{U}_{3} \mathrm{O}_{8}$ from all underground mines $(12,400,000)$ (DOE, 1983).

If we include the Schwartzwalder, the additional cost per pound of $U_{3} 0_{8}$, spread across all $\mathrm{U}_{3} \mathrm{O}_{8}$ production represented in our study, is increased to $\$ 0.50$ per pound $\mathrm{U}_{3} \mathrm{O}_{8}$ as follows:

Annualized Cost

Increased Land Value

Increased Structures Value

Increased Relocation Expenses (non-Indian)

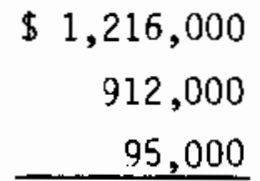

$\$ 2,223,000$

The increased cost, if applied only to the Schwartzwalder production would of course, be much higher on a unit cost basis.

\section{Associated Facilities}

As part of the on-site field examination, other land uses and associated facilities were noted around the mine vicinities. Generally, the primary uses for lands surrounding most mine sites are for mining, mineral exploration, stock grazing, and unconfined recreation. Vegetation is usually sparse, consisting of low shrubs, range grasses and occasional trees.

One important exception to this general site description is the Schwartzwalder mine near Denver, Colorado. The population, land status and land value data is not included in the final tabulation because the mine is not considered by the EPA to be typical of the uranium industry. This uranium deposit is of the vein-type versus the dominant disseminated, sedimentary/sandstone-type. Also the Schwartzwalder is located near a large metropolitan area where land can be subdivided or purchased for mountain resort homes. This makes land values extremely high compared to the average per acre 
price of range land at other sites. In addition, the mine is located in an isolated mountainous region. For these reasons, the Schwartzwalder mine is not included in this study.

Each area was also reviewed regarding its status under the BLM Intensive Wilderness Inventories. These BLM reports were last issued in 1980-81, giving detailed descriptions of state-wide study areas along with state-wide plotted maps. Appendix E gives the mine-by-mine summary of land use and associated facility information, grouped by state. 


\subsection{ECONOMIC PROFILE}

This section will provide an economic profile of the uranium industry, as broken down into three major categories: 1) demand, 2) supply, and

3) financial characteristics. Industry-wide issues are addressed and analyzed in order to understand the mechanisms involved in the uranium market place. This understanding is necessary for proper interpretation and appreciation of industry forecasts made later in this report.

\subsection{DEMAND}

\section{1 .1 Key Determinants}

This section on the components of demand will begin with an overview of the present nuclear policy, and then proceed to discuss the key determinants of demand.

\section{Nuclear Energy Policy}

The outlook of the uranium industry is related to the future of nuclear power, and the nuclear power industry is directly influenced by government energy policy. Thus, an overview of the present policy will set the stage for further discussion of demand deteminants.

President Reagan gave his statement of nuclear energy policy on October 8 , 1981 (complete statement given in App. F). As described by Hanrahan (1982), Deputy Director of the Office of Policy, Planning and Analysis for the Department of Energy, "this Administration's national energy policy is quite different from any we have had in recent years. Instead of treating energy as an isolated entity, this Administration recognizes its role as a key commodity that is part of the overall economy. Energy policy is, therefore, treated in the context of an overall plan to revitalize the economy, and is one part of the President's Program for Economic Recovery". Limiting the government's intervention is recognized as important while the private sector increases its role in energy production and consumption. 
Hancahan (1982) continues by emphasizing that the Administration is dedicated to free market principles; that a shift has been made in our national energy policy away from Federal planning and control and toward a free enterprise system. This shift has been towards removal of price and allocation controls. In summing, the emphasis of this Administration's energy policy is toward "energy security" with production and preparedness as key elements. The role of the marketplace is considered critical.

\section{Demand Determinants}

The key determinants of uranium demand and thus the outlook for the uranium industry are primarily and directly related to the future of nuclear power. However, the current and forecasted capacity of nuclear reactors is not solely responsible in building the demand equation. The demand for uranium is further complicated by many other uncertainties and variables that are beyond the control of users (utilities and others) and producers. Kovisars (1979) has compiled a list of such uncertainties affecting uranium demand projections. (Table 4.1)

The Uranium Institute (Bonny \& Fulton, 1982) has expressed the demand for uranium in mathematical terms:

$$
\text { Demand }=\text { Consumption }+ \text { Designed Change in Inventory }
$$

where

$$
\text { Consumption = f(GWe, Capacity Factor, Uranium Utilization) }
$$

In their demand forecasts, two basic methodologies are used: 1) a calculation based on nuclear reactor fuel requirements and, 2) a calculation based on estimates of enrichment capacities. The determinants of uranium demand will be discussed as related to forecasting such demand based on nuclear power capacities. The elements of the demand equation will be keyed out and described in greater detail. 
TABLE 4.1. Uncertainties Affecting Demand Projections

Growth of World Economies

- stability

- goals of developed vs. LDC countries

Development of Energy Usage

- elasticity of energy vs. income/economic performance

- technical and conversation variables

Interfuel Competition

- energy economics

- resource base for development

- hydrocarbon alternatives

- political/social pressures

- electrical share of total energy usage

Nuclear Power Capacity Growth

- system load factors

- coal/nuclear economics

- political/social constraints

- nonproliferation concerns

- waste disposal

- radiation health

Reactor Design

- uranium fuel cycle

- LWR

- HWR

- gas-cooled

- alternative fuels

- thorium cycle

- mixed-oxide reactors

- breeder

- once-through vs. closed cycle

- capacity factor

- availability

- utilization

Enrichment Criteria

- unenriched; low-enriched; high-enriched fuel

- enrichment plant design

- diffusion

- centrifuge

- jet-nozzle

- laser

- tails assay level

- contracting-delivery practice and requirenents

Recycling

- availability

- scale and timing

Inventory Decisions

- working inventories

- user inventories

- government inventories

Source: Kovisars (1979) 
Nuclear Power Capacity. The first issue of discussion is nuclear power capacity. This topic covers the GWe portion of the demand equation. Sharp reductions in forecasts of nuclear power capacity have prevailed recently (see Section 4.1.2). These reductions have resulted from technical, economic and political/socjal factors.

The strongest factor or constraint on the development of nuclear power as cited by the Uranium Institute (1979; and Bonny \& Fulton, 1982) are economic factors, especially the general economic growth of the economy. The growth rate has been considerably lower than assumed since the nuclear industry was founded. This translates into a lower overall electrical demand and thus a lower nuclear power share of that total. Another economic factor is high interest rates. High rates can force utilities to delay or cancel construction of new nuclear power plants due to the high cost of capital.

The Uranium Institute (1979) cites opposition to nuclear power as the second most important factor or constraint on nuclear power development. The whole question of nuclear acceptability and safety has resulted in many obstacles to the nuclear industry in implementing already planned programs as well as new plans.

Capacity Factor and Uranium Utilization. Assuming a viable and growing nuclear power capacity, variations in operating parameters of reactors and enrichment plants can have a profound effect on uranium demand. Topics here cover the "capacity factor" and "uranium utilization" components of the demand equation given earlier.

The capacity or load factor of nuclear reactors refers to the average capacity utilized in a year, expressed as a percentage of the plant's design capacity. A $65 \%$ load factor is assumed. The higher the factor, the greater will be the amount of fuel used up, and thus the greater will be the demand (Jranium Institute, 1981). The capacity factor can change due to environinental regulations, operating experience and/or technological developments. These changes could probably have as much as a $10 \%$ effect on the dernand level--in either direction (Bonny and Fulton, 1982). 
Changes in the level of uranium utilization can also have an effect on uranium market developments. Beginning at the enrichment stage, the tails assay could have a dramatic effect on demand. The tails assay is described as the concentration of fissile U-235 remaining in the reject stream at the government enrichment facility (Uranium Institute, 1981). This assay is an operating decision which has a direct impact on the uranium feed requirements. Tails assays have ranged between 0.18 and $0.38 \%$. The elasticity of feed requirements to the tails assay level is about 0.35 . So for a $25 \%$ change in the tails assay (e.g., from 0.20 to $0.25 \%$ ), uranium feed will increase by about 9\% (Kovisars, 1979).

Assuming a particular tails assay, fuel load characteristics become the next issue in uranium utilization. Fuel load refers to the amount of uranium required to operate a given nuclear reactor (Uranium Institute, 1981). This amount could change as fuel technology improves. Increasing fuel burn-up reduces uranium consumption during a reactors lifetime. However, the main concern of utilities which used to be to minimize uranium consumption is now changing to other goals. These goals include increasing reactor operating flexibility, minimizing costs of the total fuel cycle, and/or improving the ease and economics of spent fuel management (Bonny and Fulton, 1982). These different goals can have both individual and combined effects on uranium requirements.

Other Factors. Other important influences on demand include: stock piling or inventory (by government and utilities), reprocessing of spent fuel policy, development of alternative fuel cycles and reactor designs, processing lead times, nuclear reactor construction lead times, and import/export issues.

\subsubsection{Trends in Energy Consumption}

Projections of installed nuclear capacity have been made yearly by a variety of government and private sources. Table 4.2 shows the projections presented at the Annual Uranium Industry Seminars in Grand Junction, Colorado for the years 1975 through 1981. 
TABLE 4.2. Projections of Installed Nuclear Capacity (GWe) Presented at Annual Uranium Industry Seminar

\begin{tabular}{|c|c|c|c|}
\hline $\begin{array}{l}\text { Seminar } \\
\text { Year }\end{array}$ & Source & $\begin{array}{l}\text { Projected } \\
1985 \\
\end{array}$ & $\begin{array}{r}\text { end of year } \\
1990 \\
\end{array}$ \\
\hline 1975 & $\begin{array}{c}\operatorname{ERDA}^{(\mathrm{a})} \\
\text { (Hanrahan, 1975) }\end{array}$ & $\begin{array}{c}\text { high/mod-mod/low-low } \\
205-185-160\end{array}$ & $\begin{array}{c}\mathrm{high} / \mathrm{mod}-\bmod / 1 \text { ow- } 1 \text { ow } \\
385-340-285\end{array}$ \\
\hline 1976 & $\begin{array}{c}\text { ERDA } \\
\text { (Hanrahan, 1976) }\end{array}$ & $\begin{array}{l}\text { high-mid- low } \\
166-145-127\end{array}$ & $\begin{array}{l}\text { high-mid-10w } \\
290-250-195\end{array}$ \\
\hline 1977 & $\begin{array}{c}\operatorname{DOE}^{(b)} \\
\text { (Brown and } \\
\text { Williamson, 1977) }\end{array}$ & $\begin{array}{l}\text { with NEP(C) } \\
127 \\
\text { without NEP } \\
127\end{array}$ & $\begin{array}{l}\text { with NEP } \\
195 \\
\text { without NEP } \\
210\end{array}$ \\
\hline 1978 & $\begin{array}{c}\text { DOE/EIA }(\mathrm{d}) \\
(\mathrm{Cl} \text { ark and } \\
\text { Reynolds, 1978) }\end{array}$ & $\begin{array}{l}\text { nigh-mid-10w } \\
122-111-100\end{array}$ & $\begin{array}{l}\text { high-mid-1 ow } \\
192-172-157\end{array}$ \\
\hline 1979 & $\begin{array}{c}\text { EIA } 7 / 79 \\
\text { EIA } 10 / 79 \\
\text { (Clark and } \\
\text { Reynolds, 1979) }\end{array}$ & $\begin{array}{l}\text { high-mid-1 ow } \\
\text { 118-114-102 } \\
\text { high-mid-1 ow } \\
113-106-95\end{array}$ & $\begin{array}{c}\text { high-mid-low } \\
171-152-142 \\
\text { high-mid-1 ow } \\
155-140-129\end{array}$ \\
\hline 1980 & $\begin{array}{l}\text { ElA Spring } 80 \\
\text { OURE }^{\text {(e) }} 6 / 80 \\
\text { (Staggs, } 1980 \text { ) }\end{array}$ & $\begin{array}{c}\text { high-mid-low } \\
\text { 109-98-86 } \\
\text { high-mid-1 ow } \\
105-96-85\end{array}$ & $\begin{array}{l}\text { high-mid-low } \\
139-127-121 \\
\text { high-mid-1 ow } \\
140-129-125\end{array}$ \\
\hline 1981 & $\begin{array}{c}\operatorname{OUEA}^{(f)} \\
\text { (Staggs, 1981) }\end{array}$ & $\begin{array}{c}\text { high-mid-low } \\
99-90-80\end{array}$ & $\begin{array}{l}\text { nigh-mid-low } \\
126-122-117\end{array}$ \\
\hline
\end{tabular}

(a) Energy Research and Development Administration

(b) Department of Energy

(c) National Energy Policy

(d) Energy Information Administration

(e) Office of Uranium Resources and Enrichment

(f) Office of Uranium Enrichment and Assessment

The early projections have been optimistic. All projections of capacity installed by 1985 and 1990 were decreased by at least fifty percent between 1975 and 1981. In fact the high nuclear growth projections for 1990 have fallen by more than two thirds. 
Current DOE/EIA projections for installed nuclear capacity and total electric utility capacity are shown in Table 4.3. For comparison Table 4.4 shows similar forecasts made in 1975 by the Energy Research and Development Administration. The tables show a moderate decrease in the projections of total electric generating capacity over the past seven years. But, the interesting figures are those of the projected nuclear capacity as a percentage of the total generating capacity. A decrease in the projected values of over fifty percent is indicated for both the 1985 and 1990 forecasts. Thus, it is clear that the reductions in the projections of installed nuclear capacity are not, for the most part, due to a lowering of the forecast for total electric utility capacity; but in fact are due to the problems as discussed in section 3.7 .

TABLE 4.3. Current Projection (1983) of Installed Nuclear and Total Electric Utility Capacity (in gigawatts) by DOE/EIA

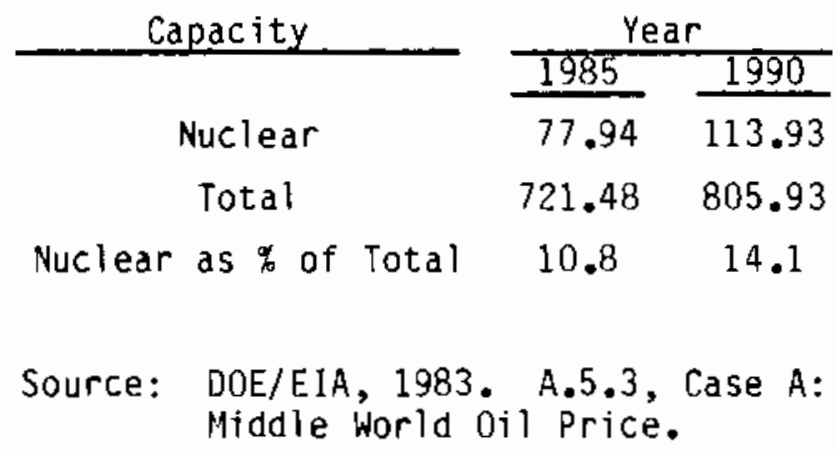

TABLE 4.4. Previous Projection (1975) of Installed Nuclear and Total Electric Generating Capacity (in gigawatts) by ERDA

$\begin{array}{cccc}\text { Capacity } & \frac{\text { Year }}{1985} & \frac{1990}{340} \\ \text { Nuclear (moderate/low) } & 185 & 340 \\ \text { Total (moderate/10w) } & 800 & 1040 \\ \text { Nuclear as of Total } & 23.1 & 32.7 \\ \text { Source: Hanrahan, 1975. Tabies } 2 \& 3 & 3 \\ & \text { Moderate Growth-Low Case. }\end{array}$




\subsubsection{Uranium Pricing}

Uranium prices and the mechanics behind price formation have developed over a four decade span. The uranium market, which began under total government control has now transferred into a private market. Following is a discussion of the development of the uranium market and its pricing policy, beginning with the Atomic Energy Commission (AEC) programs, and concluding with the current private market situation. Market pricing mechanisms will form the conclusion.

\section{Prices Under AEC}

The AEC began a uranium procurement program in 1948 by guaranteeing ore prices and negotiating concentrate prices in individual contracts with uranium mills. The period 1948-1962 was known as the "incentive period". Ore prices were guaranteed as published in a series of circulars. As discussed in Charles River Associates, Inc. (1977), circulars 3 and 4 stated that the government would give a facilities and development allowance and a premium for high grade ore containing above 0.15-0.25\% $\cup_{3} 0_{g}$ in addition to a base price (Table 4.5). These prices were effective through mid-1948 when Circular 5 was issued.

Circular 5 provided for higher base prices. Al so the development allowance was extended to lower-grade ores. Revisions came in 1951 as Circulars 5 (revised) and 6 (Table 4.6). These increased the base prices and allowances again and added a production bonus. The goal of the AEC was to encourage a wide search for new and lower grade deposits. Circular 5 (revised) was so designed to offer prices favoring lower grade ores. It was effective through March 1962. The production bonus of Circular 6 expired in March, 1960. Table 4.7 reflects the average value of ore purchased beginning 1957 (data not availiable prior to 1957 ).

Concentrate prices paid by the AEC for the same period are shown in Table 4.8. They rose between the years 1948 and 1953, after which they declined through 1962.

In 1956, the AEC announced a new fixed price of $\$ 8.00$ per pound concentrate to go into effect in 1962. The price, although below the price being paid in 1956, still proved to be an incentive for exploration and production. 
TABLE 4.5. Circulars 3 and 4: Price Schedule for Colorado Plateau Uranium From Carnotite and Roscoelite Ores

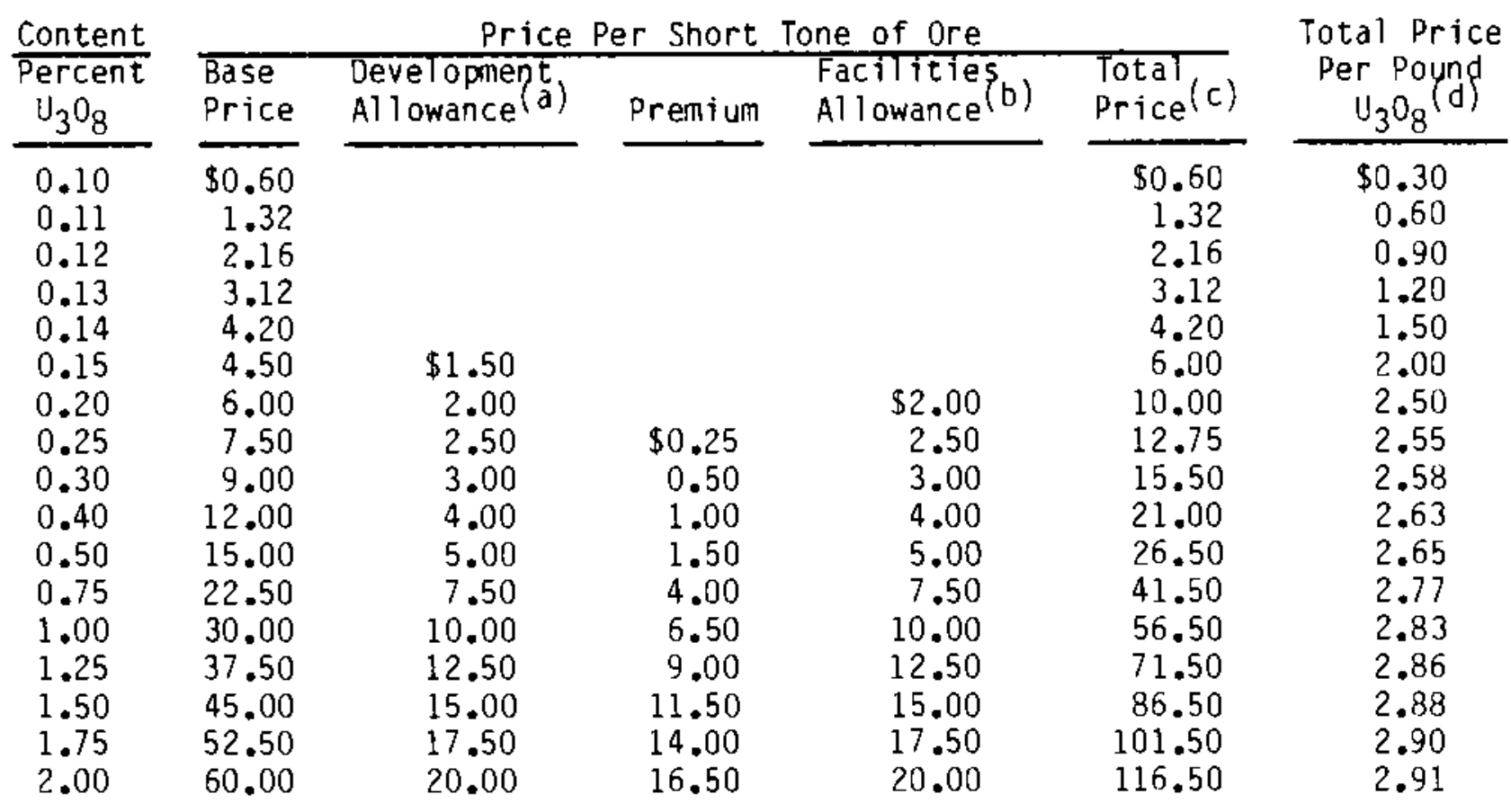

(a) To be spent only for maintaining and increasing the development reserves of uranium ores.

(b) To be spent only for reopening and equipping closed mines and increasing the production facilities of mines in operation.

(c) Excludes a haulage allowance of 6 cents per mile to a maximum of 100 miles and prices for vanadium contained in the ores.

(d) Calculated: Total price per short ton ore $\div\left(2000\right.$ lbs/short ton $\times U_{3} 0_{8}$ content, percent) $=$ Total price per pound contained $\mathrm{U}_{3} \mathrm{O}_{8}$.

Source: BOM, Minerals Yearbook, "Uranium, Radium and Thorium," 1948, p. 1269 as found in Charles River Associates Inc., 1977. p.9-17.

In late 1958, the AEC then announced an allocation program designed to limit its purchase obligations at the $\$ 8.00$ price.

The next AEC procurement program (1962-1966) saw the end of guaranteed minimum ore prices. Table 4.7 shows the average value of ore through this period.

During this 1962-1966 period, concentrate purchased by the AEC remained at the $\$ 8.00$ per pound $\mathrm{U}_{3} 0_{8}$. As stated above, the $\$ 8.00$ value was announced in 1956 and went into effect in 1962 (refer to Table 4.8). 
TABLE 4.6. Circular 5 (Revised) and Circular 6: Schedule of Prices For Uranium Ore (a) (dollars)

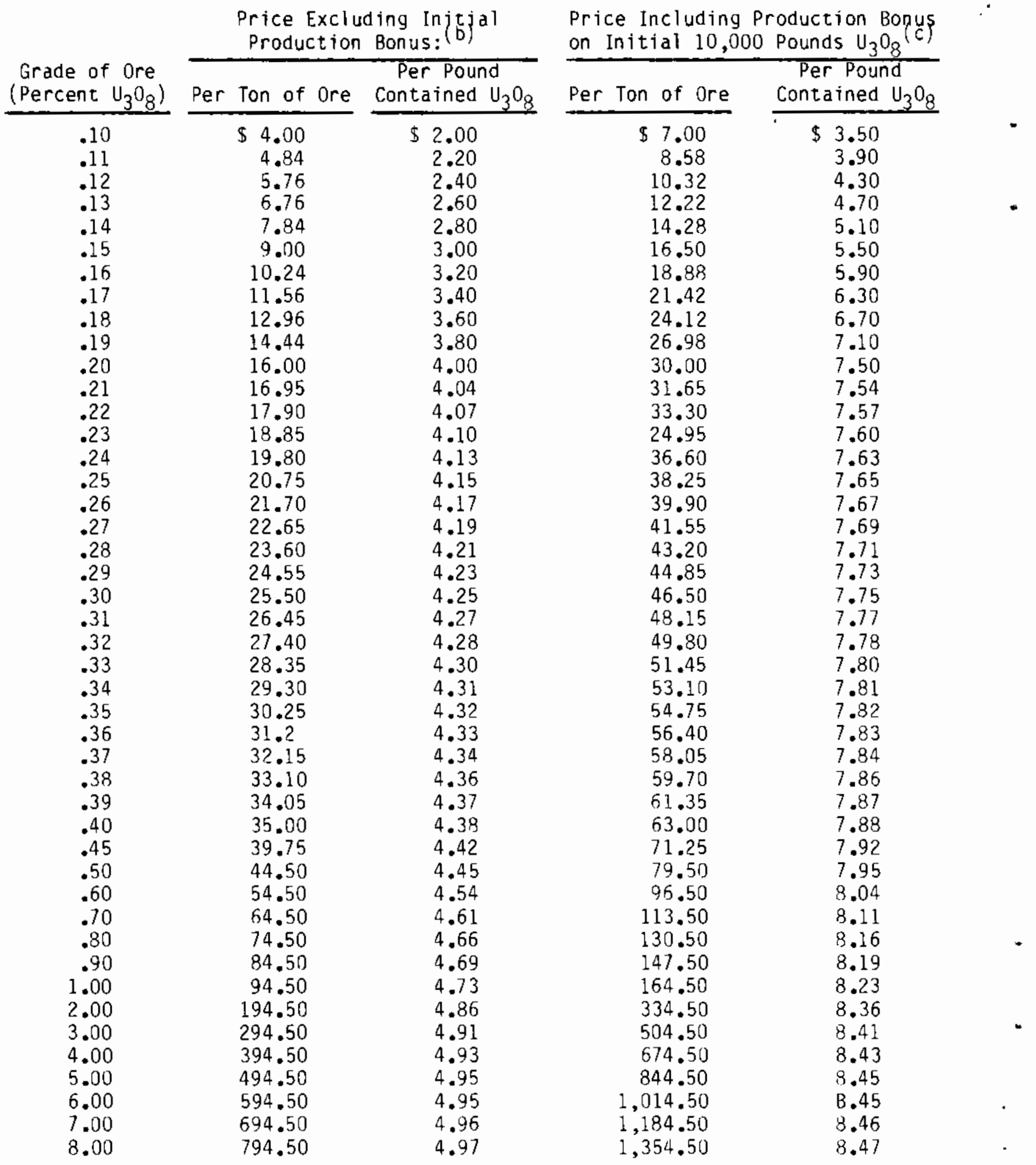


TABLE 4.6. (Contd)

\begin{tabular}{|c|c|c|c|c|}
\hline \multirow[b]{2}{*}{$\begin{array}{l}\text { Grade of Ore } \\
\text { (Percent } \mathrm{U}_{3} 0_{8} \text { ) }\end{array}$} & \multicolumn{2}{|c|}{$\begin{array}{l}\text { Price Excluding Injtjal } \\
\text { Production Bonus: }\end{array}$} & \multicolumn{2}{|c|}{$\begin{array}{l}\text { Price Including Production } 80 \text { nus } \\
\text { on Initial } 10,000 \text { Pounds } \mathrm{I}_{3} \mathrm{O}_{8}(\mathrm{c})\end{array}$} \\
\hline & Per Ton of Ore & $\begin{array}{l}\text { Per Pound } \\
\text { Contained } \mathrm{U}_{3} \mathrm{O}_{8}\end{array}$ & Per Ton of Ore & $\begin{array}{c}\text { Per Pound } \\
\text { Contained } \mathrm{U}_{3} \mathrm{O}_{8}\end{array}$ \\
\hline $\begin{array}{r}9.00 \\
10.00\end{array}$ & $\begin{array}{r}\$ 894.50 \\
994.50\end{array}$ & $\begin{array}{r}\$ 4.97 \\
4.97\end{array}$ & $\begin{array}{r}\$ 1,524.50 \\
1,694.50\end{array}$ & $\begin{array}{r}\$ 8.47 \\
8.47\end{array}$ \\
\hline
\end{tabular}

(a) All prices exclude haulage allowances of 6 cents per mile to a maximum of 100 miles and payments for vanadium ores.

(b) Includes base price, grade premium and mine development allowance.

(c) Bonus not to exceed a maximum of $\$ 35,000$ per property.

Source: Calculated from Circular 5 (revised), reprinted in GAO, op. cit., in JCAE, Hearings, $1962, \mathrm{p} .300$, and information in BOM, Minerals Yearbook, "Uranium," 1951, pp. 1305-1306. As found in Charles River Associates Inc., 1977, pp. 9-19 to 9-21.

Special "stretch-out" arrangements were offered by the AEC through 1970. This allowed mills to defer concentrate deliveries scheduled for 1963-1966 until 1967-1968. The AEC then agreed to purchase additional amounts equal to the differed amounts during 1969-1970. By this time a private market for uranium had developed and the AEC procurement policies showed less importance in determining $\mathrm{U}_{3} \mathrm{O}_{8}$ prices. (Charles River Associates, Inc., 1977).

Private Market Prices

The private uranium market began in the late 1960s. Its beginning was characterized by depressed prices, but after mid-1973, a marked upswing in market conditions caused prices to rise rapidly. Table 4.9 gives NUEXCO exchange values beginning August 1968 to December, 1982. These commercial prices are also shown graphically (Figure 4.1) in both actual and 1982 dollars. AEC prices (active and 1982 dollars) are also plotted for comparison. The table and figure show how prices began to increase in 1973 and then sharply in 1974 and 1975.

Increased contracting in 1976 caused another sharp increase in price. More contracts were signed in the first half of 1976 than in all of 1975. (Gordon, 1977). The price per pound $\mathrm{U}_{3} \mathrm{O}_{8}$ was $\$ 41.00$ for spot delivery at 
TABLE 4.7. Average Value of Bre

\begin{tabular}{lcc} 
Year $(\mathrm{a})$ & $\begin{array}{l}\text { Dollars Per } \\
\text { Ton of Ore }(\mathrm{b})\end{array}$ & $\begin{array}{c}\text { Dollars Per Pound } \\
\text { Contained } U_{3} \mathrm{O}_{8}(\mathrm{c})\end{array}$ \\
\cline { 2 - 3 } 1957 & $22.36(\mathrm{~d})$ & 4.19 \\
1958 & $22.50(\mathrm{~d})$ & 4.15 \\
1959 & $20.38(\mathrm{~d})$ & 4.08 \\
1960 & $19.04(\mathrm{e})$ & 4.02 \\
1961 & $18.44(\mathrm{e})$ & 4.00 \\
1962 & $19.61(\mathrm{e})$ & 4.05 \\
1963 & $20.04(\mathrm{e})$ & 4.05 \\
1964 & $20.52(\mathrm{e})$ & 4.08 \\
1965 & $19.23(\mathrm{e})$ & 4.04 \\
1966 & $17.81(\mathrm{e})$ & 3.90 \\
1967 & $15.25(\mathrm{e})$ & 3.90 \\
1968 & 14.50 & 3.87 \\
1969 & 16.02 & 3.50 \\
1970 & 14.68 & 3.71 \\
1971 & 15.57 & \\
\hline
\end{tabular}

(a) Prior to 1957, production data were classified and no annual data are reported in the Minerals Yearbooks. The BOM did not estimate this series for earlier years when the data became available in 1955 and 1956. Value of ore production/shipments is not reported separately, in a form comparable to earlier years, after 1971 .

(b) Oerived by dividing ore production (or ore shipped) into estimated ore value. The calculations are based on unrevised production data.

(c) Derived by dividing total value of ore by recoverable $\mathrm{U}_{3} \mathrm{O}_{8}$ content of ore (in pounds), prior to adjustment for milling recovery rates.

(d) F.0.B. mines.

(e) Based on quantity of ore shipped, rather than ore production.

Sources: Derived from data on ore production and total ore value provided by the AEC to the BOM, and reported in BOM, Minerals Yearbook, "Uranium," 1957 through 1971. As Found in Charles River Associates, Inc., 1977, op. 9-14 to 9-15. 
TABLE 4.8. AEC Domestic Concentrate Purchases: Amount, Cost and Average Price

AEC Purchases as Percent of Total Concentrate

\begin{tabular}{|c|c|c|c|c|}
\hline Fiscal Year & Tons $\mathrm{U}_{3} \mathrm{O}_{8}$ & $\begin{array}{l}\text { Cost to } \mathrm{AEC} \\
\left(\text { in } \$ 10^{6}\right)\end{array}$ & $\begin{array}{c}\text { Average Price/ib. } \\
U_{3} 0_{8}(\text { in } \$)\end{array}$ & $\begin{array}{l}\text { of Total Concentrate } \\
\text { Production (percent of } \\
\left.\text { tons } \mathrm{U}_{3} \mathrm{O}_{8}\right)^{(\mathrm{b})}\end{array}$ \\
\hline $\begin{array}{l}1948 \\
1949 \\
1950 \\
1951 \\
1952 \\
1953 \\
1954 \\
1955 \\
1956 \\
1957 \\
1958 \\
1959 \\
1960 \\
1961\end{array}$ & $\begin{array}{r}116 \\
115 \\
323 \\
639 \\
824 \\
968 \\
1,435 \\
2,125 \\
4,179 \\
7,505 \\
10,078 \\
15,029 \\
16,394 \\
17,646\end{array}$ & $\begin{array}{r}1.7 \\
2.0 \\
5.9 \\
12.9 \\
18.6 \\
23.9 \\
35.2 \\
52.1 \\
96.2 \\
157.4 \\
190.5 \\
274.1 \\
287.0 \\
299.8\end{array}$ & $\begin{array}{r}7.14 \\
8.53 \\
9.11 \\
10.10 \\
11.28 \\
12.35 \\
12.27 \\
12.25 \\
11.51 \\
10.49 \\
9.45 \\
9.12 \\
8.75 \\
8.50\end{array}$ & \\
\hline Sub-Total & 77,646 & $1,457.2$ & Average 9.42 & \\
\hline $\begin{array}{l}1962 \\
1963 \\
1964 \\
1965 \\
1966\end{array}$ & $\begin{array}{l}17,244 \\
15,752 \\
12,607 \\
11,240 \\
10,178\end{array}$ & $\begin{array}{l}281.0 \\
246.2 \\
201.7 \\
179.8 \\
162.8\end{array}$ & $\begin{array}{l}8.15 \\
7.82 \\
8.00 \\
8.00 \\
8.00\end{array}$ & $99 \%$ \\
\hline Sub-Total & 67,021 & $1,071.6$ & Average 7.99 & \\
\hline $\begin{array}{l}1967 \\
1968 \\
1969 \\
1970 \\
1971 \text { (a) }\end{array}$ & $\begin{array}{l}8,902 \\
7,937 \\
7,124 \\
4,010 \\
1,295\end{array}$ & $\begin{array}{r}142.4 \\
127.0 \\
99.6 \\
46.0 \\
14.3\end{array}$ & $\begin{array}{l}8.00 \\
8.00 \\
6.99 \\
5.74 \\
5.54\end{array}$ & $\begin{array}{l}83 \\
66 \\
60 \\
32 \\
20\end{array}$ \\
\hline Sub-Tota 1 & 29,268 & 429.4 & Average 7.34 & \\
\hline TOTAL & 173,655 & $2,958.2$ & Average 8.52 & \\
\hline
\end{tabular}

(a) Through December 1970 in Fiscal year 1971 .

(b) Purchases in a given fiscal year refer to the receipt of concentrate by the AEC in accordance with contract provisions. They do not refer to the amounts "contracted for" by the AEC in any given year.

Source: ERDA, Statistical Data of the Uranium Industry, GJ0-100. January 1976, p. 11. As found in Charles River Associates, Inc., 1977, pp. 9-11 to $9-12$. 
TABLE 4.9. Historical Exchange values

(U.S. Dollars/Tb $\mathrm{U}_{3} \mathrm{O}_{3}$ )

\begin{tabular}{|c|c|c|c|c|c|c|c|c|c|c|c|c|}
\hline Ir & in. & . & r. & r. & $y$ & n. & uul. & g. & p. & t. & v. & ec. \\
\hline 88 & -- & -- & -- & - & -- & $\rightarrow$ & -- & 6.35 & 6.35 & 6.40 & 6.45 & 5.50 \\
\hline & - & & 6.10 & & & 0 & & & & & 6.15 & \\
\hline & 6.3 & 6.3 & 6.30 & & & & & & & & & 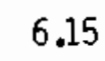 \\
\hline & 6.2 & 6.2 & 20 & 6.2 & & 6.0 & & & 9 & & & 5.95 \\
\hline & 5.95 & 5. & 5.95 & 5. & 5. & 5.95 & & 5.95 & 5.95 & & & 5.95 \\
\hline & 5.95 & & 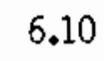 & 6.20 & & & & 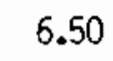 & .50 & & & 7.00 \\
\hline & 7.7 & & 8.00 & & & 0.50 & & & 2.50 & & & 5.00 \\
\hline & 00 & $m$ & 0 & 20.00 & 1.00 & 0 & & 0 &, 00 & & & 55.00 \\
\hline & 35.20 & 370 & 39.25 & 40.00 & .00 & 40.00 & 0 & 40.40 & 1.00 & 0 & 0 & 41.00 \\
\hline & 41.35 & & 41.60 & 60 & & 42.25 & & .25 & 2.40 & 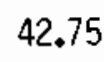 & & +3.20 \\
\hline & 42.90 & 43.25 & 25 & 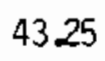 & & 43.40 & & & 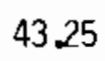 & & & 43.25 \\
\hline & 43.25 & 43.25 & .25 & 25 & 43.25 & 0 & & 0 & 20 & 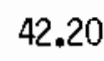 & & .75 \\
\hline & 40.00 & $3 x$ & 5.00 & 32.00 & & 31.50 & & 00 & .50 & & & 27.00 \\
\hline & 25.00 & 25. & 25.00 & 25.00 & & 24.25 & & 23.50 & 23.50 & 23.50 & 23.50 & 23.50 \\
\hline & 300 & .00 & 2.50 & 20.75 & 20.50 & 9.25 & 18.75 & 17.00 & 17.00 & 17.50 & 19.75 & 2 \\
\hline
\end{tabular}

Source: Nuexco Exchange values as faund in Nuclear News Magazine, Nuclear Fuel Newsletters, and Nuexco price quotes.

yearend 1976. However, many producers were not benefiting from this price increase due to locked-in contracts at earlier lower prices. This occurred at a time when exploration and production costs were increasing. (Charles River Associates, Inc., 1977).

1977-1978 saw relatively stable prices with the NUEXCO exchange value increasing to $\$ 43.20$ by yearend 1977 and $\$ 43.25$ in 1973 . The outstanding feature in the 1977 uranium market, as described by white (1978), was buyers' concern about security of supply. Buyers were convinced that the best hedge against supply disruptions was to purchase and hold inventory, either as $U_{3} 0_{8}$ concentrate or as reserves in the ground. The continued stability in 1978 reflected a balance in the market place. However, 1979 saw the beginning of erosion of the exchange value for $\mathrm{U}_{3} \mathrm{O}_{8}$ concentrate. Between 1979-1981 


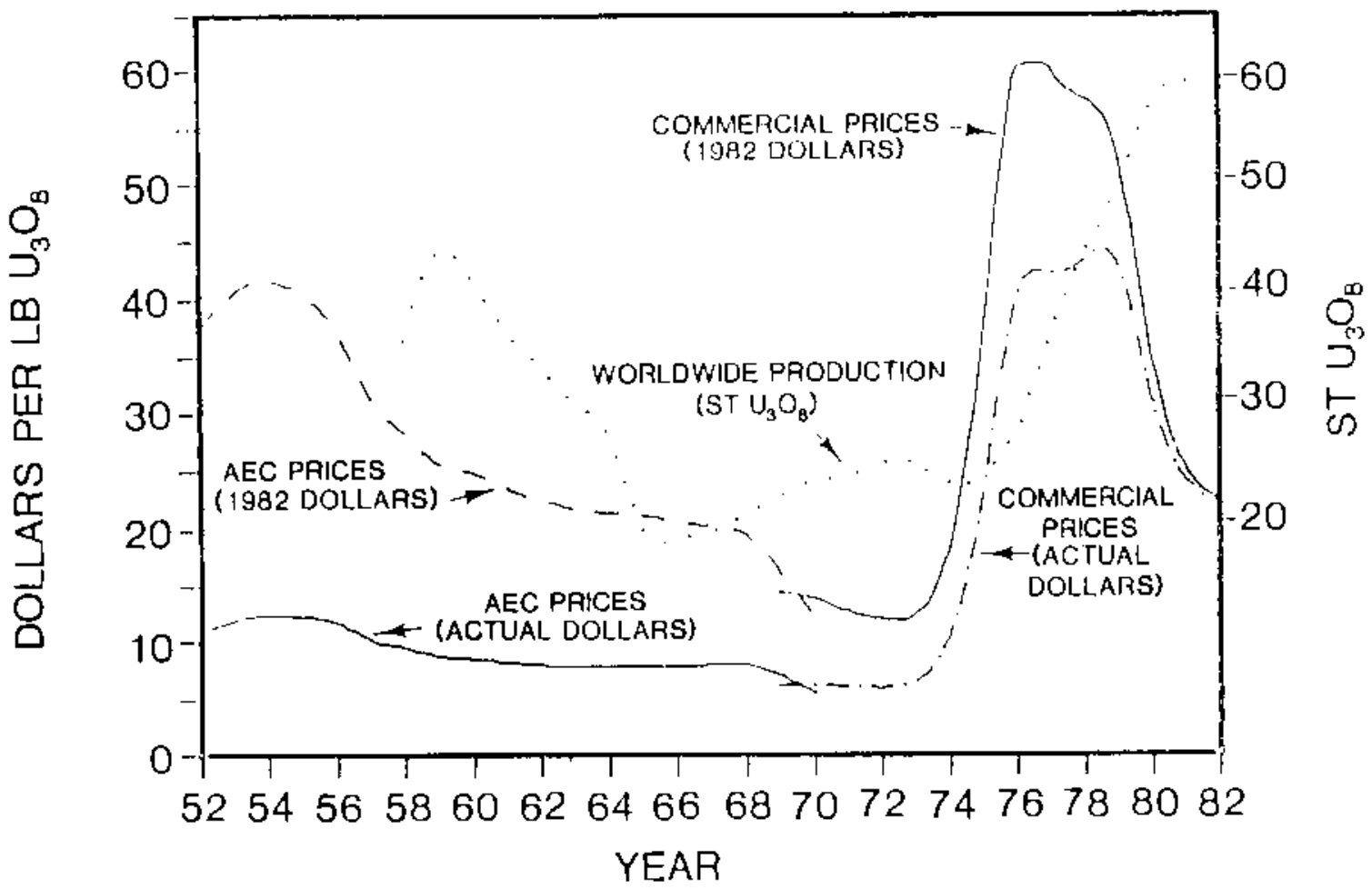

FIGURE 4.1. History of $\mathrm{U}_{3} \mathrm{O}_{8}$ Prices

Source: Hahne, F. L. III, 1982. "Future Uranium Prices," paper presented at Uranium Colloquium $V$, Grand Junction Colorado, Oct. 6-7, 1982.

inclusively, the constant dollar decrease was $20 \%, 45 \%$ and $25 \%$ respectively. The downward spiral finally ended in September, 1982 at $\$ 17.00$ per pound $\mathrm{U}_{3} \mathrm{O}_{8}$ - Yearend 1982 saw a slight rebound to $\$ 20.25$ per pound $\mathrm{U}_{3} \mathrm{O}_{8}$.

The decline, as described by white, $(1979,1980,1981,1982,1983)$, was due to an imbalance between production and consumption. In 1980, material available for sale was often in excess of purchase requests by ratios of $4: 1$ or more. In 1981, production committed for delivery under existing supply contracts exceeded actual reactor consumption by almost $100 \%$. This resulted in buyer inventories increasing by nearly 50 million pounds $\mathrm{U}_{3} \mathrm{O}_{8}$ in 1981 . And finally, in 1982, the continued price decline resulted from an aggressive selling campaign by both U.S. and non-U.S. utilities and producers. The pressure from this great inventory liquidation caused prices to break sharply from a somewhat stabilized yearend 1981 price. 


\section{Pricing Mechanisms}

Having discussed the uranium market and uranium price development, closing remarks will be made on market pricing techniques or mechanisms. Basically, market pricing refers to that mechanism by which two parties attempt to deal with an uncertain future uranium price situation by setting forth procedures to arrive at a contract price that reflects market conditions at the time of delivery. This mechanism usually operates for intermediate and long term contracts versus spot sales.

Many contract approaches have been tried and used by buyers and sellers. The first such contract which helped to give market pricing credibility in the U.S., was termed a negotiated approach and occurred between the Tennessee Valley Authority and Rio Algom (a Canadian firm) in 1974. It was a 12 year agreement involving 17 million pounds of $\mathrm{U}_{3} \mathrm{O}_{8}$.

In the negotiated approach, price negotiations are begun prior to an agreed upon year of delivery. The lead negotiating time can be from several years to only months prior to delivery. Objectives are established, negotiation sequences are laid out, and if necessary, experts are used to expedite results. There are several variations on this approach, differing mainly in form and manner of price setting.

A second major market pricing mechanism is the reference approach. Here, the market price is determined based on published and public reference sources for prices. These include the NUEXCO exchange value, Metals Week, American Metal Market, U.S. Department of Energy price data, the NUKEM Price Quote, and the Uranium Price Information Service data by the Nuclear Assurance Corporation. There are also several variations of the reference approach.

\subsubsection{Elasticity of Demand}

In the short and intermediate terms, the demand for uranium is relatively price-inelastic (Aucoin, 1982). That is, the demand is insensitive to the price of the commodity. This inelasticity stems from the fact that there are no substitutes for uranium as a fuel in the present nuclear fission-type reactor. Present demand for uranium is directly linked to the fuel requirements of those reactors that are presently on-line. 
Future demand will depend on both those reactors that are under construction and completed and those being planned. The number of planned reactors will depend on the demand for nuclear-powered electricity which is linked to the overall growth of electrical demand and cost of alternative energy sources.

The reductions in projected nuclear power capacity are in part due to the many problems facing the industry as discussed in Section 3.7. Two important points are emphasized here that will effect utilities' future nuclear ambitions, and thus the outlook for uranium demand. These are: 1) the future financial conditions of the utilities and 2) the relative costs and acceptability of competing energy sources including other nuclear technology (Bock, 1978). Because of long lead times for power facility construction, a utility's financial coverage is usually lowered and it financial rating reduced, thus resulting in a higher cost of capital acquisition. Among the competing energy sources, coal is viewed as the chief alternative to nuclear power.

Returning to the present, once a nuclear power plant is constructed, an adequate fuel supply is vital for operation. The relative cost of fuel is small compared to construction and finance costs. Once construction is started or completed, the cost of the fuel supply is no issue. It must be secured at any cost. Thus, demand becomes insensitive to price or is inelastic.

In the long run however, demand for uranium may not be totally inelastic. This is due to choices made by utilities on future energy sources for electricity generation versus the nuclear option in light of the utilities' perceptions of future uranium fuel prices and other reasons cited above.

\subsection{SUPPLY}

\subsubsection{Key Determinants}

Making estimates of future uranium supply beyond the short-term is as uncertain as forecasting demand. Reliable or accurate estimates can be made only when the entire market is understood from the economic, physical, social, and political aspects. These aspects are composed of many key determinants that dictate how the industry will respond to market forces of demand. These key determinants will be discussed as they relate to uranium supply. 
As described by Bonny and Fulton (1982) supply is a function of two basic economic factors-price and cost. This can be written as:

$$
\text { production }=f \text { (uranium price, mining costs). }
$$

This relationship can be complex. When market prices for uranium are low for a lengthy period relative to production costs, some high-cost mines are forced to work higher grade ores. As well, they must become more cost efficient by implementing cost-saving strategies. Some mines may be forced to extend production capabilities in order to reduce unit costs (Uranium Institute 1981).

The uranium industry has shown its ability to respond to market forces. Increased demand for uranium and price incentives have spurred production at sufficient rates. However, because of such a rapid build-up of supply capacity over the last decade, oversupply has resulted. This oversupply has upset the supply/demand balance and since 1979, prices have plummeted by as much as $45 \%$ per year (refer to Section 4.1.4--Uranium Pricing). What was once an expanding industry is now one of a series of mine/mill suspensions and closures.

Such economic forces are perhaps the most important factors in determining future uranium production and supply. There must be a production incentive plus a perception of a long-term uranium market in order for producers to plan future operations. The size of the market will depend on the overall rate of economic growth and the associated growth in demand for electricity. The market will also depend on the ability of utilities to finance new nuclear power plants.

Current financial conditions in the mining industry are very important as well when looking at future supply. This is so because today's company profits will be used in part to finance future exploration and operations. However, during periods of low market prices, management may not make favorable exploration/investment decisions. Shortage of financial resources and expertise could result; a situation which could be hard to reverse later. Also, because of long lead times required for facility development, future demand may not be met adequately even if exploration/investment decisions were favorable at some later date (Uranium Institute 1981). 
There are certain short-term characteristics that are important in understanding the uranium supply market. These are the physical constraints on production. They include mine/mill operating characteristics and the size and nature of the uranium ore deposits. Short-term increases or decreases in production are flexible within the limits of these constraints.

At the underground mine, increasing output is more difficult than for an open-pit operation because of problems resulting from limited haulage, hoisting and ventilation capacity, and from using inflexible underground mining methods (Uranium Institute 1981). Skilled labor shortages due to mine remoteness and exposure-related health concerns are also constraints to expansion.

At the mill, capacity utilization and recovery rates are important. Increased mill output can be achieved by a step-up in utilization, either up to or exceeding designed capacity, for short periods. Increased throughput, however, tends to negatively effect the uranium recovery rate (Uranium Institute, 1981).

The physical nature of deposits also determines the rates at which uranium can be produced. Ore grade is a given of nature, but cut-off grade may be raised to increase production (by having a higher uranium concentration in the ore) and lower unit cost. However, high grading will eventually effect the longer-term ore mining schedule and shorten the mine's life.

The combined effects of all these economic and physical constraints on the future supply of uranium are: longer lead times for developing new projects, a slower rate of production build-up towards design capacity, and continually higher extraction costs (Uranium Institute 1979). But while these constraints have a marked influence on supply, other nontechnical factors effect the market as well.

Other constraints encompass political and social issues. Government intervention in the uranium mining and milling industry has increased over the years due in large part to these issues. As described by the Uranium Institute (1981) these include: environmental impacts of uranium mining and milling operations, land and mineral rights of local population groups, ownership and foreign participation, nuclear non-proliferation policies, and the effects of uranium inports upon the uranium mining industry. 


\subsubsection{Interindustry Organization and Market}

The Nuclear Fuel Cycle

The existing nuclear industry or the nuclear fuel cycle is characterized by "front-end" and "back-end" activities (see Table 4.10). The front-end of the cycle refers to all activities which result in the production of fabricated fuel elements. Front-end operations include exploration, mining, milling, aqueous conversion, enrichment, and fabrication. Power production is the focus of the nuclear fuel cycle. The back-end of the cycle currently refers to the interim-storage of spent fuel assemblies. Reprocessing of spent fuel has been deferred because of complex technical/political issues. Effective implementation of recycling spent fuel is unlikely until the late 1980's or later (EIA 1977).

Ownership in the nuclear fuel cycle is very diversified with a declining number of independent producers. Entrants include petroleum firms, minerals companies, utilities, and in the case of enrichment, the federal government. Joint ventures play an important role in many front-end activities (DOE 1982).

Several factors have contributed to the integrated nature of the uraniurn industry. Major incentives include the AEC weapons program, nuclear power generation, the development of self-supply ventures, and the cancellation of nuclear projects. The following sections discuss the influence of these events on the structure of the nuclear supply industry. Examples of vertical and horizontal integration are also provided.

\section{AEC Weapons Program}

Several of the front-end operations developed as a result of the AEC weapons program in the 1950s and 1960s. Most supply facilities were owned by small independent firms. Custom manufacture of uranium products was not uncominon to meet the unique needs of weapons testing.

The end of the weapons program left the uranium supply industry in disinal condition. In 1958, AEC announced that no reserves developed before November would be eligible for procurement. The AEC then stretched out its purchase program to prevent collapse of the industry (EPRI 1977). Market activity almost halted and many development programs were abandoned (EIA, 1977). 
TABLE 4.10. Current Nuclear Fuel Cycle ACtivities

\section{Activity}

Exploration and Development

Mining

Milling

$\stackrel{\leftrightarrow}{\sim}$ Conversion

Enrichment

\section{Omership}

Private and Federal

Private

Producers

Private

Producers

Private

Producers

DOE owned but operated by private industry under

contract

\section{Description}

Uranium mineralization is delineated and accessed. Major steps include land acquisition, surface drilling, and reserve development.

Ore is removed from sand or limestone rock using underground or surface methods. It is transported in bulk by truck or rail to nearby milling facillities (Schneider 1979).

Uranium ore is converted to a concentrated ammonium diuranate powder called yellowcake. Yellowcake consists of 74.2 wt\% U as uranium diuranate (Schneider 1979).

IJranium concentrate is converted to the volatile compound UF $_{6}$ and shipped as a solid to enrichment facilities. The processes used are the hexafluor process and the "wet" process. The fissionable isotope U-235 is $0.7 \%$ of the total uranium content (Schneider 1979). Plants are located in Metropolis, Illinois, and Sequoyah, Oklahoma (AEC 1974).

UF $_{6}$ is isotopically enriched in U-235 to the range of 2 to $4 \%$ for use a reactor fuel. The $U_{6}$ is shipped as a solid to fuel fabrication plants. In addition to the enriched UF product, the gaseous diffusion plant produces $U_{6}$ depleted in U-235 called tails. The current assay of tails is $0.25 \% \mathrm{U}-235$. The tails are stored as solid UF at the plant for future use. Plans are located in 0ak Ridge, Tennessee; Portsinouth, Ohio; and Paducah, Kentucky (Schneider 1979). 
Activity

Fuel

Fabrication

Power Plants $\stackrel{\infty}{N}$

Spent Fuel

Storage
Ownership

Private

Processors

Public

Utilities

Utilities

\section{Description}

Fuel fabrication plants manufacture fuel assemblies from $\mathrm{UF}_{6}$. The $\mathrm{UF}_{6}$ is converted to $\mathrm{U}_{2}$. The $\mathrm{UO}_{2}$ is pelletized and sinterred to the desired density and loaded into fuel rods. of the ten comercial plants licensed in 1971, only three produced completed reactor fuel assemblies. Four other plants used enriched $\mathrm{UF}_{6}$ feed to produce $\mathrm{U}_{2}$ power or pellets. The three used $\mathrm{UO}_{2}$ powder or pellets to produce fuel assemblies. When necessary, powdered $\mathrm{UO}_{2}$ is transported in steel pails by truck (AEC 1974).

Reactors produce electricity from the fissionable U-235 isotope in fuel assemblies. As of Dec, 31, 1982, there were 95 PWRs, 49 BWRs, and 3 other nuclear power plants domestically in operational or constructional phases (Nuclear News 1983). In a PWR, the heat produced by the nuclear reaction is removed by a pressurized water system. Heat exchangers transfer heat from the pressurized water to produce steam. A BWR boils water, directly to produce steain for the generators.

Spent fuei is usually stored at reactor sites. At the end of 1977 , approximately 3000 pounds of spent fuel was discharged of which $10 \%$ was reprocessed, $15 \%$ was stored at reprocessing facilities, and the remainder was stored at the utility. All reprocessing of spent fuel terminated in 1971 (EIA 1977). 
In 1965 through 1970, exploration became active again. During this period, oil and mineral companies actively purchased or merged with small independent producers. Control was probably gained by accident as a result of oil and gas drilling. Plans for nuclear power projects could also have provided incentives for these investments despite oversupply and low prices. By 1972 , oil companies held $50 \%$ of proven reserves.

As large mining and exploration firms appeared, many mine/mill complexes were constructed. Major oil and mining companies currently active in milling include Kerr-McGee, Exxon, Getty $0 i 1$, and ARCO.

0il and mineral firms entering front-end fuel cycle activities is an example of horizontal integration. Horizontal integration occurs when a company produces more than one product or merges with a competitor (EPRI 1977). Nuclear Power Generation

Nuclear power development required the construction of fabrication plants, nuclear reactors, and back-end facilities. Major public utilities and corporations were responsible for reactor projects. Large diversified corporations constructed fabrication plants. Projects were heavily regulated and highly technical with significant environmental, financial, and legal responsibilities. Entry by small firms was virtually impossible because of the complexity of the facilities.

By 1974, nuclear power was a commercial reality but aggressive exploration efforts were still not underway. The market changed to a buyer's market, and the price for uranium tripled in a matter of two years. Low contract prices, however, set a few years previous were not conducive to aggressive exploration efforts. Exploration efforts were also inhibited by uncertainties in nuclear requirements. Variations in enrichment and recycle policies alone could have resulted in a $40 \%$ spread in potential demand for uranium (Stoller 1977). Factors affecting the demand for uranium are shown in Table 4.1.

Shortages were expected if exploration efforts were not pursued since future demand was in excess of known reserves. Exploration and reserve development require lead times of at least 10 and 6 years, respectively. Long lead times and the rapid growth of the projected power industry made these activities potential limiters of uranium supply (Doiron 1978). 


\section{Utility Self-Supply Programs}

A utility requires a guaranteed supply of nuclear fuel to be a reliable source of power. In fact, state regulatory commissions were demanding that lifetime supplies of uranium be assured before approving a reactor project. Utilities were encouraged, to participate in exploration and development to assure long-term supply and price control (Stoller 1977).

A utility entering a supply sequence to lower costs and assure supply is an example of vertical integration. By 1980 , about $40 \%$ of all American nuclear electric utilities had participated in uranium supply ventures. In some cases, the utility took only a limited role by financing the venture, while the minerals company was responsible for planning the program (Mommsen 1980).

The earlier a participant enters a supply chain, the higher the risks. Risks include barren drilling, technical problems, delays, poor production, high costs, or complete failure. These risks are far beyond those of normal procurement. Money spent on barren reserves must be included in overall production costs.

Power Plant Delays Cancellations

Over the last few years, uranium supply has again become a buyer's market. Forecasts for nuclear power demand had decreased to $40 \%$ of 1978 levels and several nuclear projects have been delayed or cancelled (Heiks 1982). Development efforts of the 1 ate 70 s resulted in over-capacity, large inventories, and low profits (Nuclear News, 1982).

Many utilities feel their performance in supply activities has not been good. Many have dropped out and utility commissions are discouraging supply ventures, since sufficient capacity currently exists to supply fuel projections until at least 1995. Most utilities are dissatisfied with the costs of production due to heavy exploration expenditures and rising capital costs for mine development (Mommsen 1980).

Effects of Integration

The level of corporate investinent in the uranium industry has steadily increased over the last two decades. Both horizontal and vertical integration have significantly reduced the number of independent enterprises except as 
joint partners. Exxon is an example of a large domestic enterprise with diversified energy investments. Exxon has substantial investments in coal, oi1, uranium, and natural gas reserves, and most of the domestic oil refining capacity. Exxon also mines and mills uranium, fabricates fuel, and has announced plans to be one. of the first private entrants into the enrichment industry.

Some officials believe that oil and minerai mergers may have detrimental effects. Exxon for example, owns oil and gas wells jointly with other large firms in the industry so that even corporate entities are not independent. When economic power is concentrated between a few large corporate entities, there are potential anti-competitive consequences. They include harmful supply conditions, abusive pricing policies, and less entrepreneurial spirit. The political leverage of such major companies could also inhibit appropriate environmental and occupational regulations. The self-interests of interdependent sellers does not always conform to the public interest of sufficient supply at a reasonable price.

Most potential benefits result from the relatively large capital budgets of major corporations. Explorations and production efforts can be expanded despite variable market conditions. Larger budget R\&D efforts could also increase efficiencies (U.S. Congress 1975).

\subsubsection{Trends in Production and Supply}

Historical data for the uranium industry is presented in Table 4.11. The table lists ore production from mines which is received at mills and buying stations. Production is broken down between open-pit and underground mines with the average ore grade being a derived result.

Several things are obvious from the data. First, the average ore grade concentration from both types of mines has fallen with the largest decrease occurring in open-pit mines. The ore concentration taken from open-pit mines in 1982 was more than $70 \%$ less than that produced in open-pit mines during the first ten years of uranium mining in the U.S. Similarly the ore concentration from underground mines fell over the same period; but for 1982 it was more than three times the concentration from open-pit mines. Also, it can be seen that 
TABLE 4.11. Iranium Ore Produced from Mines and Received at Mills and Buying Stations(a)

\begin{tabular}{|c|c|c|c|c|c|c|c|c|c|}
\hline \multirow[b]{2}{*}{ Year } & \multicolumn{3}{|c|}{ Total Receipts } & \multicolumn{3}{|c|}{ Open-pit Production } & \multicolumn{3}{|c|}{ Underground Production } \\
\hline & $\begin{array}{l}\text { Tons } \\
\text { Bre } \times 10^{3}\end{array}$ & $\mathrm{U}_{3} 0_{8} \times 10^{3}$ & $\begin{array}{l}\text { Ore Conc\% } \\
\text { (Derived) }\end{array}$ & $\begin{array}{l}\text { Tons } \\
\text { 0re } \times 10^{3}\end{array}$ & $\begin{array}{l}\text { Tons } \\
\mathrm{U}_{3} 0_{8} \times 10^{3}\end{array}$ & $\begin{array}{l}\text { Ore Conc\% } \\
\text { (Derived) }\end{array}$ & $\begin{array}{l}\text { Tons } \\
\text { Ore } \times 10^{3} \\
\end{array}$ & $\mathrm{U}_{3} \mathrm{O}_{8} \times 10^{3}$ & $\begin{array}{l}\text { Ore Conc\% } \\
\text { (0erived) } \\
\end{array}$ \\
\hline $1948-57$ & 11,308 & 32.2 & 0.285 & 3,796 & 9.5 & 0.250 & 7,512 & 22.7 & 0.302 \\
\hline 1958 & 5,178 & 14.0 & 0.270 & 2,358 & 5.4 & 0.229 & 2,820 & 8.6 & 0.305 \\
\hline 1959 & 6,935 & 17.4 & 0.251 & 2,206 & 4.4 & 0.199 & 4,729 & 13.0 & 0.275 \\
\hline 1960 & 7,970 & 18.8 & 0.236 & 2,393 & 5.3 & 0.221 & 5,577 & 13.5 & 0.242 \\
\hline 1961 & 8,041 & 18.5 & 0.230 & 2,482 & 5.3 & 0.214 & 5,559 & 13.2 & 0.237 \\
\hline 1962 & 7,053 & 17.1 & 0.242 & 1,782 & 4.3 & 0.241 & 5,271 & 12.8 & 0.243 \\
\hline 1963 & 5,948 & 14.7 & 0.247 & 1,879 & 4.4 & 0.234 & 4,069 & 10.3 & 0.253 \\
\hline 1964 & 5,297 & 13.9 & 0.262 & 1,537 & 3.4 & 0.221 & 3,760 & 10.5 & 0.279 \\
\hline 1965 & 4,376 & 10.4 & 0.238 & 1,243 & 3.0 & 0.241 & 3,133 & 7.4 & 0.236 \\
\hline 1966 & 4,329 & 9.9 & 0.229 & 1,333 & 3.1 & 0.233 & 2,996 & 6.8 & 0.227 \\
\hline 1967 & 5,272 & 10.7 & 0.203 & 1,593 & 3.2 & 0.201 & $3,67 \mathrm{~g}$ & .7 .5 & 0.204 \\
\hline 1968 & 6,448 & 12.6 & 0.195 & 2,366 & 4.6 & 0.194 & 4,082 & 8.0 & 0.196 \\
\hline 1969 & 5,904 & 12.3 & 0.208 & 2,173 & 5.2 & 0.239 & 3,731 & 7.1 & 0.190 \\
\hline 1970 & 6,324 & 12.8 & 0.202 & 2,801 & 5.9 & 0.211 & 3,523 & 6.9 & 0.196 \\
\hline 1971 & 6,279 & 12.9 & 0.205 & 3,284 & 7.0 & 0.213 & 2,995 & 5.9 & 0.197 \\
\hline 1972 & 6,418 & 13.7 & 0.213 & 3,887 & 8.1 & 0.208 & 2,531 & 5.6 & 0.221 \\
\hline
\end{tabular}


TABLE 4.11. (Contd)

\begin{tabular}{|c|c|c|c|c|c|c|c|c|c|}
\hline \multirow[b]{2}{*}{ Year } & \multicolumn{3}{|c|}{ Total Receipts } & \multicolumn{3}{|c|}{ Open-Pit Production } & \multicolumn{3}{|c|}{ Underground Production } \\
\hline & $\begin{array}{l}\text { Tons } \\
\text { Ore } \times 10^{3}\end{array}$ & $\mathrm{U}_{3} \mathrm{O}_{8}^{\text {Tons }} \times 10^{3}$ & $\begin{array}{l}\text { Ore Conc\% } \\
\text { (Derived) }\end{array}$ & $\begin{array}{l}\text { Tons } \\
\text { Ore } \times 10^{3}\end{array}$ & $\begin{array}{l}\text { Tons } \\
\mathrm{U}_{3} \mathrm{O}_{8} \times 10^{3} \\
\end{array}$ & $\begin{array}{l}\text { Ore Conc\% } \\
\text { (Derived) }\end{array}$ & $\begin{array}{l}\text { Tons } \\
\text { Ore } \times 10^{3}\end{array}$ & $\mathrm{U}_{3} \mathrm{O}_{8}^{\text {Tons }} \times 10^{3}$ & $\begin{array}{l}\text { Ore Conc\% } \\
\text { (Derived) }\end{array}$ \\
\hline 1973 & 6,537 & 13.6 & 0.208 & 4,544 & 8.6 & 0.189 & 1,993 & 5.0 & 0.251 \\
\hline 1974 & 7,027 & 12.4 & 0.176 & 4,216 & 7.3 & 0.173 & 2,811 & 5.1 & 0.181 \\
\hline 1975 & 7,057 & 12.0 & 0.170 & 4,247 & 6.7 & 0.158 & 2,810 & 5.3 & 0.189 \\
\hline 1976 & 8,608 & 13.5 & 0.157 & 4,673 & 6.8 & 0.146 & 3,935 & 6.7 & 0.170 \\
\hline 1977 & 10,325 & 15.9 & 0.154 & 5,578 & 7.6 & 0.136 & 4,747 & 8.3 & 0.175 \\
\hline 1978 & 14,342 & 18.8 & 0.131 & 8,237 & 9.6 & 0.117 & 6,105 & 9.2 & 0.151 \\
\hline 1979 & 15,011 & 15.7 & 0.105 & 9,655 & 9.4 & 0.097 & 5,356 & 6.3 & 0.118 \\
\hline 1980 & 16,745 & 20.0 & 0.119 & 10,394 & 10.4 & 0.100 & 6,351 & 9.6 & 0.151 \\
\hline 1981 & 13,665 & 15.6 & 0.114 & 8,436 & 7.0 & 0.083 & 5,229 & 8.6 & 0.164 \\
\hline 1982 & 8,313 & 10.1 & 0.121 & 5,504 & 3.9 & 0.071 & 2,809 & 6.2 & 0.221 \\
\hline TOTALS & 210.710 & 389.5 & 0.185 & 102,597 & 159.4 & 0.155 & 108,113 & 230.1 & 0.213 \\
\hline
\end{tabular}

(a) Source: II.S. Department of Energy (1983). Statistical Data of the Uranium Industry. Table 1-6. (Mined ore; does not include production from mine water, in-situ leach liquor, heap-leach solutions, byproducts, and miscellaneous low-grade ore from old mine dumps.) 
even though the ore concentration from open-pit mines is falling more rapidly than from underground mines the production is not. Since 1971 there has been a larger percentage of ore production from open-pit than underground mines. In 1982 almost two-thirds of the ore production came from open-pit mines while this amounted to less than forty percent of the years production of $\mathrm{U}_{3} \mathrm{O}_{8}$. Finally, the information in the table reveals that only two years after a peak production of 20,000 tons of $U_{3} 0_{8}$ in 1980 production of concentrate from domestic mines had fallen almost fifty percent. This decrease represents the steepest production drop in the history of the domestic uranium industry (as deduced from the data).

Many factors contributed to this abrupt decrease in production. In 1976 the price of $U_{3} \mathrm{O}_{8}$ hit an all time high in real terms and by mid-1978 the price peaked in nominal terms at $\$ 43.40 / 1 b$ of $U_{3} 0_{8}$. The price began to decline in nominal terms as well about mid-1979 (Hahne 1982). This price drop continued until September 1982 when the price bottomed at $\$ 17.00 /$ ib of $U_{3} 0_{8}$ (Nuclear News, 1983c), which is the lowest price in real terms since 1973. The price drop cut producer margins significantly making it unprofitable for most producers to continue mining operations and many producers found it more profitable to meet contract obligations by making purchases on the spot market than by new production.

Inventories during the period 1979 to 1981 increased from about 90 to 130 million pounds of $\mathrm{U}_{3} \mathrm{O}_{8}$ which is more than three years of projected domestic reactor demand. At the same time projections of installed domestic nuclear capacity had continued to fall (see Table 4.2). The result of this was a large inventory sell off by utilities and others causing increased pressure on producers to reduce output.

\subsubsection{Facility Utilization-Operating Capacity, Production Lifetime}

\section{Operating Capacity}

The term "facility" can refer to the mining operation or milling unit. The U.S. Department of Energy reports annual statistics on the activities of both facilities. However, for the mining side, data is given as total ore in concentrate produced from all mines of each type (i.e., underground, open-pit, 
other) or by state. No indication is given as to nominal mine capacity (minespecific or generically) or percent mine utilization. Designed mine capacities can sometimes be gotten from various published documents, but information is rarely up-to-date or complete. Therefore, a detailed breakout of data on nominal and operating capacities on a mine-specific basis is not possible.

The U.S. Department of Energy (1983), however, has recently issued information on nominal capacity and percent utilization for uranium mills. These mills are generally considered to be production centers--with the capacity of the mill representing the supporting mines. As of January 1, 1983, there were 14 conventional uranium mills operating. These mills had a total rated capacity of 33,650 tons of ore per day (Table 4.12). Ore actually milled from these 14 units in 1982 equalled 21,510 tons per day for a 1982 operating level of $63.9 \%$ of capacity. In addition, there were 10 licensed mills not operating as of January 1, 1983, but which milled some ore throughout 1982 . Their operating level was 16.3\% of capacity. The total 1982 operating level was $45.4 \%$ of capacity (Table 4.13). The 1983 estimated total operating level is about $35 \%$ of capacity. (a)

Specific information on mine and/or mill operating levels, as reported in company annual and Form $10-K$ reports, is as follows:

- Rio Algom, Ltd.--Lisbon Mine operating on a $50 \%$ rate since October 1981. The integrated mine/mill complex has a nominal mill capacity of 700 tons ore/day.

- Homestake Mining Co.--Two of their Grants, New Mexico mines have been placed on standby in 1982. The Homestake mill is operating at a reduced level of 8 days on, 6 days off since April, 1982.

- UNC, Resources, Inc.--As of Apri1, 1982, all UNC uranium production was suspended and facilities were put on standby.

- Kerr-McGee Corp.--7 of 11 uranium mines in New Mexico are operating at reduced levels while the remaining 4 have been put on standby. Two surface mines in Wyoming have also been placed on standby.

(a) Information from, U.S. Department of Energy, Grand Junction Area Office, Colorado. 
TABLE 4.12. U.S. Uranium Processing Plants: Operating as of January 1, 1983

Conventional Mills
Atlas Minerals Corporation
Bear Creek Uranium Company
Onevron Resources company
Cotter Corporation
Energy Fuels Nuclear, Inc.
Exxon Minerals Company
Horestake Mining Company
Kerr-MtGee Nuclear Corporation
Minerais Exploration Company
Pathfinder Mines Corporation
Pathfinder Mines Corporation
Petrotomics Company
Rio Algan Corporation
Union Carbide Corporation
Total

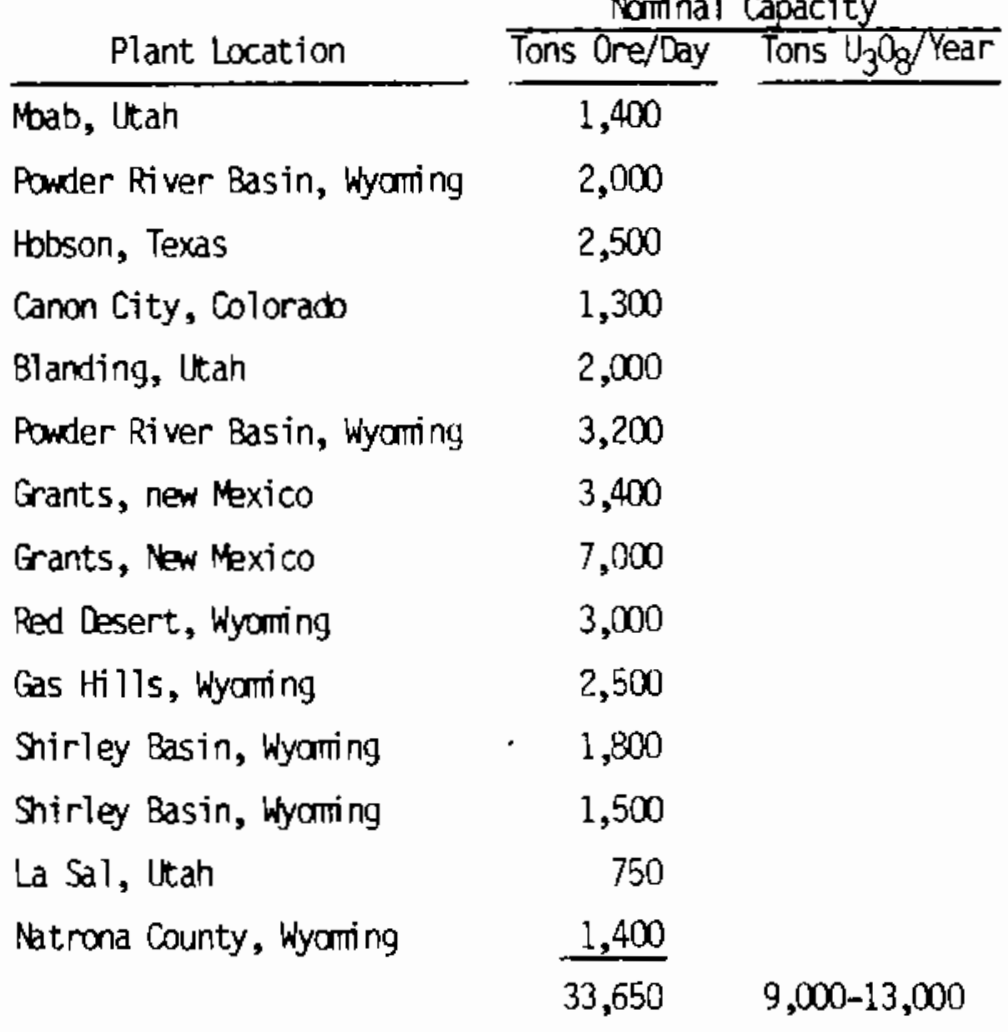

Source: U.S. DOE (1983), Table VII-2 p. 42.

TABLE 4.13. Conventional Mill Status Summary for 1982

\begin{tabular}{|c|c|c|c|c|}
\hline Mill Status & $\begin{array}{c}\text { Nunber of } \\
\text { Mills }\end{array}$ & $\begin{array}{l}\text { Rated Capacity } \\
\text { (tons ore/day) }\end{array}$ & $\begin{array}{l}\text { Milled in } 1982 \\
\text { (tons ore/day) }\end{array}$ & $\begin{array}{l}1982 \text { Operating } \\
\text { Level as Percent } \\
\text { of Capacity }\end{array}$ \\
\hline operating on $1 / 1 / 83$ & 14 & 33,650 & 21,510 & 63.9 \\
\hline Not Operating on $1 / 1 / 83$ & 10 & 21,400 & 3,490 & 16.3 \\
\hline Totals & 24 & 55,050 & 25,000 & 45.4 \\
\hline
\end{tabular}

Source: U.S. DOE (1983), Table VII-3, p. 45. 


\section{Production Lifetime}

A "typical lifetime" statement about a uranium mine, is difficult to make. This is so because uranium ore deposits are so varied as to size, grade, depth, and difficulty of mining. A uranium mine life is also impacted by price, mining technology improvements and by-product production. However, some general comments can be made.

The San Juan Basin Regional Uranium Study (1980) lists uranium mine assumptions that were used in their study as a basis on which to gauge future uranium mine development in the San Juan Basin region (Table 4.14). These assumptions, based on industry and government sources, were made concerning the average new uranium mine going into production in the basin from 1980 on. The average life expectancy is given as 20 years.

\section{TABLE 4.14. Average New Mine}

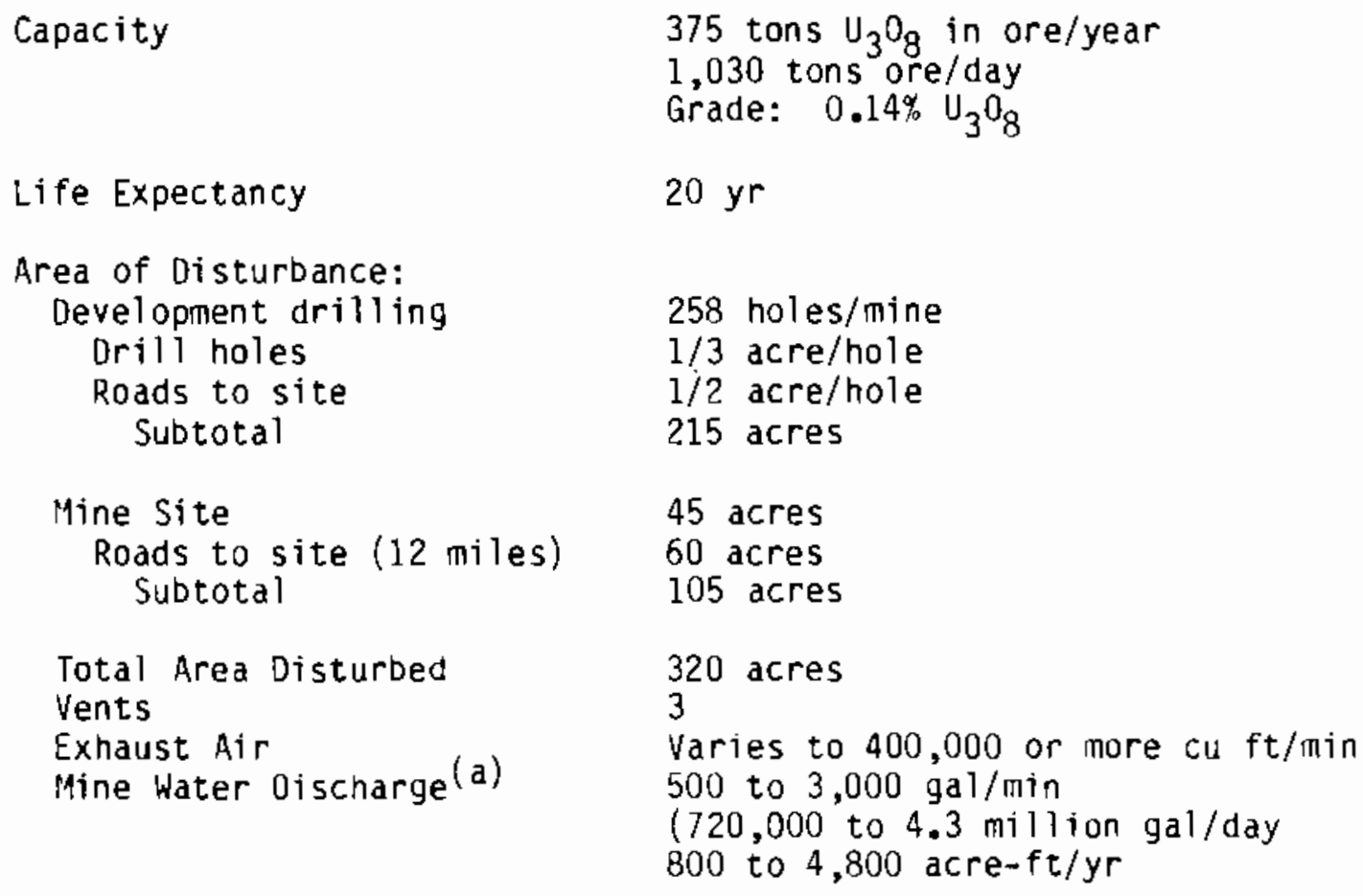

(a) Academic use only. U.S. Geological Survey model uses other methods. Source: San Juan Basin Regional Uranium Study (1980) p. II-30. 
Other examples of mine lives as taken from company annual reports and other sources are given for comparison:

Kerr-lickee Corp.:

- Section 19 Mine has been operating for 7 years

- Section $30 E$ Mine has been operating for 24 years

- Section 30w Mine has been operating for 14 years

- Section 35 Mine has been operating for 12 years

- Section 36 Mine has been operating for 23 years

Homestake Mining Co.:

- Section 23 Mine has been operating for 24 years

- Section 25 Mine has been operating for 24 years

Gulf Mineral Resources:

- Mt. Taylor Mine has 20-year expected life

- Mariano Lake Mine has had a 5-year total life (now depleted)

Phillips Petroleum Co.:

- Nose Rock has 20-year expected life

\subsubsection{Productivity and Employment Trends}

\section{Productivity}

Mining technology and methods have had few major changes since the early 1950s. The industry though has seen improvements in the productivity of existing procedures plus has benefited from general improvements in inining equipinent and methods--especially blasting and nauling (Charles River Associates, Inc. 1977).

The impact of technological change can be manifested in increased labor productivity over time. However, due to other contributing factors, it is cautioned that such labor productivity may not be the best ineasure of technological change. Table 4.15 gives data on output per man-shift. The table shows that labor productivity increased substantially after the 1950s. This was a period of mechanization and increasing uranium mining experience (Charles River Associates, Inc. 1977). 
TABLE 4.15. Estimates of Labor Productivity in Uranium Mining

\begin{tabular}{|c|c|c|}
\hline Date & Output Per Unit Labor Input & Source \\
\hline $\begin{array}{l}1954-56 \\
\text { (Colorado Plateau) }\end{array}$ & $1.0-3.1$ tons ore/man-shift & Bureau of Mines (a) \\
\hline $\begin{array}{l}\text { Pre- } 1960 \\
\text { Colorado Plateau) }\end{array}$ & $2.1-13.3$ tons ore/man-shift & Bureau of Mines $(b)$ \\
\hline 1969 & Open-pit: 16 tons ore/man-shift & Facer $(c)$ \\
\hline $\begin{array}{l}\text { Late 1960s, } \\
\text { early } 1970 \mathrm{~s}\end{array}$ & $\begin{array}{l}\text { Underground: approximately } 8 \text { tons } \\
\text { ore/man shift (as much as } 20 \text { tons } \\
\text { ore/man-shift in larger mines) }\end{array}$ & Facer \\
\hline 1974 & Open-pit: 30 tons ore/man-shift & Facer \\
\hline 1974 & $\begin{array}{l}\text { Underground: approximately } 7 \text { tons } \\
\text { ore/man-shift ( } 10 \text { tons ore/man-shift } \\
\text { in larger mines) }\end{array}$ & Facer \\
\hline 1978 & Underground: averages 6 tons ore/man-shift & (Facer 1978) \\
\hline 1979 & Underground: nearing $B$ tons ore/man-shift & (Facer 1979) \\
\hline 1980 & Underground: averaging 8 tons/man-shift & (Anderson 1980) \\
\hline
\end{tabular}

(a) W. L. Dare, Uranium Mining Methods and Costs at Several Mines on the Colorado plateau, B0M, Information Circular 8015, 1961 .

(b) BOM, Mineral Factor and Problems, 1960, p. 928.

(c) J. Fred Facer, Jr., "Production Statistics," in ERDA, Uranium Industry Seminar, Grand Junction, Colorado, 1975; p. 152.

Source: As found in Charles River Associates, Inc. (1977). Added--Facer (1978, p. 189; 1979, p. 195) and Anderson (1980, p. 125).

Other data from the U.S. Department of Energy on output per employee is given in Table 4.16. It is noted that these figures do not reveal changes in the average duration of employment and thus may not accurately reflect labor productivity trends (Charles River Associates, Inc. 1977). However, the figures show that output per man was stable between 1968 and 1974 for underground mining. A sharp drop occurred in 1975, but productivity has since risen to 1.3 tons/man for 1982 . 
TABLE 4.16. Changes in Output Per Man in Open-Pit and Underground Mines

\begin{tabular}{|c|c|c|c|c|c|c|}
\hline & \multicolumn{3}{|c|}{ Open Pit } & \multicolumn{3}{|c|}{ Underground } \\
\hline & $\begin{array}{l}\text { Employment }(a) \\
\text { (number) }\end{array}$ & $\begin{array}{l}\text { Total Ore } \\
\text { Production } \\
\text { (short tons) } \\
\end{array}$ & $\begin{array}{c}\text { Output / } \\
\text { Man } \\
\end{array}$ & $\begin{array}{c}\text { Employment } \\
\text { (number) }\end{array}$ & $\begin{array}{l}\text { Total ore } \\
\text { Production } \\
\text { (short tons) }\end{array}$ & $\begin{array}{c}\text { Output/ } \\
\text { Man }\end{array}$ \\
\hline 1968 & 675 & 2366 & 3.5 & 2890 & 4082 & 1.4 \\
\hline 1969 & 956 & 2173 & 2.3 & 2865 & 3731 & 1.3 \\
\hline 1970 & 1033 & 2801 & 2.7 & 2521 & 3523 & 1.4 \\
\hline 1971 & 1122 & 3284 & 2.9 & 2236 & 2995 & 1.3 \\
\hline 1972 & 1221 & 3887 & 3.2 & 1766 & 2531 & 1.4 \\
\hline 1973 & 1167 & 4544 & 4.0 & 1552 & 1993 & 1.3 \\
\hline 1974 & 1071 & 4216 & 3.9 & 2020 & 2811 & 1.4 \\
\hline 1975 & 1274 & 4247 & 3.3 & 2927 & 2810 & 1.0 \\
\hline 1982 & 1365 & 5504 & 4.0 & 2150 & 2809 & 1.3 \\
\hline
\end{tabular}

(a) Number employed at end of year: includes miners, and service and support personnel; excludes supervisors, and technical and clerical employees. For 1975 and 1982, figures reflect average employment levels for the year.

Source: Employment: ERDA/AEC, Statistical Data of the Uranium Industry, 1969-1976; 0utput: Appendix Table A.4 as found in Charles River Associates, Inc. (1977). Added--U.S. Dept. of Energy (1983), Tables I-6 and XI-1. 
Employment Trends

Historical data on employment in the uranium industry has been kept by the U.S. Department of Energy and its predecessors. Table 4.17 gives such data for years 1973 through 1982, divided by type of activity. Total employment peaked in 1979 at 22,191 and has been falling ever since.

TABLE 4.17. Employment in the Uranium Industry

\begin{tabular}{|c|c|c|c|c|c|c|c|c|c|c|}
\hline $\begin{array}{l}\text { Type of } \\
\text { Activity }\end{array}$ & 1973 & 1974 & 1975 & 1976 & 1977 & 1978 & 1979 & 1980 & 1981 & 1982 \\
\hline Exploration & 1,557 & 1,697 & 2,049 & 2,793 & 4,140 & 4,449 & 4,066 & 3,370 & $2,300^{(a)}$ & $N_{-} A_{0}(C)$ \\
\hline Mining(b) & 3,516 & 3,928 & 5,386 & 7,603 & 11,453 & 13,338 & 14,649 & 13,298 & 8,984 & $6,242^{(d)}$ \\
\hline Milling & 1,522 & 1,668 & 2,237 & 2,727 & 3,175 & 3,615 & 3,476 & 3,251 & 2,367 & $1,956^{(d)}$ \\
\hline Totals & 6,595 & 7,293 & 9,672 & 13,123 & 18,768 & 21,402 & 22,191 & 19,919 & 13,651 & \\
\hline
\end{tabular}

(a) Estinated.

(b) Includes solution mining and by-product operations.

(c) N.A. = Nbt available.

(d) Not included are an estimated 140 contract truckers.

Source: DeVergie (1982) p. 23. 1982 data fran U.S. Dept. of Energy (1983). Table XI-1, p. 63.

Oevergie (1982) notes that the greatest relative change is seen in the exploration sector. From 1979 through 1982, employment here has plummeted by 2800 workers. Also, with the general slowdown in development work, fewer people were employed in the fields of shaft-sinking and other development functions in 1981 and 1980. Other mineral and mineral fuel industries have had substantial layoffs as well during 1981 and 1982, which makes it very difficult for the displaced uranium worker to find new jobs in their field of expertise.

\subsubsection{Imports/Exports}

Imports

As mentioned earlier, import levels have an effect on the domestic uranium industry. Import levels effect demand for uranium in the sense that if a utility can secure its reactor fuel requirements through lower-cost imported foreign supplies, then their demand for domestic higher cost uranium would be less. 
U.S. Government policy has affected import levels in this country. The U.S. Department of Energy is the only domestic processor allowed to enrich uranium concentrate for nuclear applications. Between 1964 and 1976, imports were banned. All foreign uranium enriched in the IJ.S. had to be returned to the country of origin. The ban was lifted by $10 \%$ beginning in 1977, i.e., 10\% of any U.S. utility's enriched uranium requirements could be of foreign origin. This amount increased by an additional $10 \%$ each year through 1981 . In 1982, the ceiling was $60 \%$, rising to $80 \%$ in 1983 and then vanishing altogether in 1984 (Nuclear Fuel 1981C).

Table 4.18 shows actual and allowable percentages of foreign uranium that toll enrichment customers (utilities) could have delivered for domestic end use each year. Note that domestic utilities are currentiy buying less foreign uranium than the percentage allowed by $1 \mathrm{lw}$.

TABLE 4.18. Deliveries of Uranium to DoE Enrichment Plants by Domestic Customers

\begin{tabular}{|c|c|c|c|c|c|}
\hline \multirow[b]{2}{*}{ Year } & \multicolumn{3}{|c|}{ Origin (Short tons $\mathrm{U}_{3} \mathrm{O}_{8}$ ) } & \multicolumn{2}{|c|}{ Percent Foreign } \\
\hline & U.S. & Foreign & Total & Actual & Al lowable \\
\hline 1977 & 14,240 & 703 & 14,943 & 4.7 & 10 \\
\hline 1978 & 11,957 & 728 & 12,685 & 5.7 & 15 \\
\hline 1979 & 15,436 & 1,591 & 17,027 & 9.3 & 20 \\
\hline 1980 & 11,135 & 1,241 & 12,376 & 10.0 & 30 \\
\hline 1981 & 10,067 & 1,132 & 11,199 & 10.1 & 40 \\
\hline $1977-1981$ & 62,835 & 5,395 & 68,230 & 7.9 & 22 \\
\hline
\end{tabular}

Source: U.S. Department of Energy (1982). Table XII, p. 16.

Recent action taken by the U.S. Congress, beginning in 1981, has been aimed at protecting the U.S. uranium producing industry. On March 31, 1982, the Senate approved an amendment originally proposed by Senator Domenici of New Mexico to the 1982/1983 NRC Authorization Bill (S1207). This amendment proposed an import limit of foreign uranium to $20 \%$ of required fuel for U.S. Nuclear reactors. A Senate/House conference Comittee resulted when such a provision was not included in the Houses' version of the bill (H.R. 2330) (Connor 1983). The conference committee settled on a $37.5 \%$ foreign import limit which was passed by the Senate on October 1, 1982. 
The bill called for an annual report by the DOE containing a calculation of the ratio of imports to requirements for the next 10 years. When the $37.5 \%$ Iimit was exceeded in any two consecutive years, a two-year freeze on new foreign contracting would be triggered. Also a study would be triggered of the viability of the U.S. uranium industry by the U.S. International Trade Commission and an assessment by the Department of Commerce of the national security impact of uranium imports. In addition, the DOE would be required to revise its enrichment criteria so as to enhance the use of domestic uranium (Nuclear Fuels 1982a).

Reaction to the proposed import ban was mixed arnong utility, industry, and professional people. The issue was the prime topic at the Uranium Colloquium $V$ held in Grand Junction, Colorado, in October 1982.

Paul Gilman of the Senate Subcommittee on Energy Research and Development, opened the conference by showing full support for the import restriction. He said that weakening demand resulting from reactor cancellations and deferrals were the prime cause of the current depressed state of the II.S. uranium industry, but that the threat of increased foreign imports is also affecting the U.S. industry. He gave data from the DOE's Energy Information Administration that import commitments increased by 20,200 tons $\mathrm{U}_{3} \mathrm{O}_{8}$ in 1981 and an additional 19,800 tons $\mathrm{U}_{3} \mathrm{O}_{8}$ during the first half of 1982 - an $85 \%$ share of all new commitments made by domestic buyers for the 18-month period through July 1982 (Gilman 1982).

Two utility representatives gave opposing views. Russell Hulse, of the Arizona Public Service company preferred legislatively-imposed import restrictions over self-imposed limits by utilities. However, he still thought that it would not be wise to restrict all foreign uranium so as to keep some competition between U.S. and foreign producers (Huise 1982). Louis Martin of Carolina Power and Light on the other hand, was opposed to restrictions. He said that such restrictions were overrated as being the solution to the problems of the U.S. industry but admitted that import limits may have some place in an overall program to support its existence (Martin 1982).

James Bedore of the Uranium Institute in London opposed the restrictions while Eugene Lang of Rocky Mountain Energy was in support of inport limits 
(Bedore 1982 and Lang 1982). A view was also expressed by James Munro, a representative of the Australian Western Mining Corporation, Ltd. He opposed restrictions (Munro 1982).

After discussing all arguments, the House of Representatives defeated the import restriction legislation on December 2, 1982 (Nuclear Fuel 1982b). Thus, the present import ban phase-out will continue as scheduled, ending totally in 1984.

The NRC FY-82/83 Authorization Act (of which the import issue was a part) was passed, however. This Act requires various studies of the U.S. uranium industry. The Department of Energy must determine annually between 1983 and 1992 the viability of the domestic industry using criteria adopted by September 1983 following a public rulemaking (Nuclear Fuel 1983a). As reported in the American Mining Congress Journal (1983), the AMC Lranium Policy Council has formed a working group to gather information to submit to the Department of Energy on this subject of domestic uranium industry viability. This information will be part of the DOE's report.

\section{Exports}

Export levels also have an effect on the domestic uranium industry. Export levels reflect the world market demand for domestically produced uranium. U.S. producers are and will be faced with competition from lower cost foreign suppliers. Table 4.19 gives historical data on U.S. imports and exports. Through 1975, the U.S. was a net exporter as imports were governed by 1aw. From 1975 to 1977, imports exceeded exports but from 1978 through 1980, the reverse prevailed with the U.S. becoming a net exporter of uranium. In 1981 , the U.S. again reversed and became a net importer.

\subsection{FINANCIAL CHARACTERISTICS}

\subsubsection{Capital Requirements}

This section will provide an overview of the current financial situation of the uranium/minerals industry. An introduction to types of analyses used in making mineral investment decisions will provide background for further financial discussion. nata will be given on uranium industry expenditures and a closing comment will be made on project financing from a banking perspective. 
TABLE 4.19. U.S. Imports and Exports of Uranium for Commercial Uses (tons $\mathrm{U}_{3} \mathrm{O}_{8}$ )

\begin{tabular}{lrr}
$\frac{\text { Year }}{1966}$ & $\begin{array}{r}\text { Annual } \\
\text { Imports }\end{array}$ & $\begin{array}{r}\text { Annual } \\
\text { Exports }\end{array}$ \\
\cline { 2 - 3 } 1967 & 0 & 400 \\
1968 & 0 & 700 \\
1969 & 0 & 800 \\
1970 & 0 & 500 \\
1971 & 0 & 2,100 \\
1972 & 0 & 200 \\
1973 & 0 & 100 \\
1974 & 0 & 600 \\
1975 & 0 & 1,500 \\
1976 & 700 & 500 \\
1977 & 1,800 & 600 \\
1978 & 2,800 & 2,000 \\
1979 & 2,600 & 3,400 \\
1980 & 1,500 & 3,100 \\
1981 & 1,800 & 2,900 \\
& 3,300 & 2,200
\end{tabular}

Source: IJ.S. Department of Energy. 1982. Table X, p. 14 .

Investment Decisions/Expenditures

Stermole, (19B3) simply states that "the current financial state of the mineral industry, in general, is bad... and that economic prospects for improvements in the near future are uncertain." He emphasizes that mineral commodity prices must rise so that mineral investment economics can improve and so that industry can return to a more normal level of production. Prices must rise so as to make reopening of closed mines and expansion or development of new mines economically justifiable. Product prices are seen in investment analysis as being one of the key parameters in assessing the economic potential of existing or new investments. Stermole (1983) states that "it will take significant improvement in nonprecious metal mineral commodity prices in 1983 to develop significant new mine investment interest except in very high grade ore body special situations." 
As for uranium specifically, White (1983) summarizes the 1982 uranium situation by citing the trend in U.S. exploration and development activity and the participation levels of companies. The trend in activity for uranium continued downward throughout 1982. Many programs were curtailed or completely terminated. Drilling activity was reduced dramatically. Participation by companies in the uranium business dropped considerably as well. White (1983) notes that of 15 major oil companies active early in 1982, 8 have since announced an intent to terminate all uranium activities. The other 7 have reduced activities sharply. For small mining companies and independent operators, the exodus rate is even higher.

Mineral investment decision making generally involves three types of analysis according to Stermole (1983):

- Economic analysis

- Financial analysis

- Intangible analysis.

An economic analysis considers "the relative economic merits of investment situations from a profitability viewpoint based on discounted cash flow analysis of projected investment revenues and costs." Financial analysis, however, "refers to where and how the funds for proposed investments will be obtained." And finally, intangible analysis "considers factors affecting investments but which cannot be quantified easily in economic terms." A careful distinction must be made between economic and financial analyses.

Expenditure or capital investment data is sumrarized by the Department of Energy. Table 4.20 shows uranium industry capital investment for the years 1977-1984. Capital investment for exploration and mining/milling facilities increased throughout the latter 1970s. In 1981-1982 total capital investment decreased substantially while planned expenditures will be even lower.

Financing/Capital Acquisition

Borrowing money to finance new mining ventures is an important consideration in a company's overall project analysis. If project financing cannot be secured, the project will not get off the ground regardless of its 
TABLE 4.20. Capital Investment for the Domestic Uranium Industry

Expenditures in Million Dollars Actual

\begin{tabular}{|c|c|c|c|c|c|c|c|c|}
\hline \multirow[b]{2}{*}{ Investment Category } & \multicolumn{6}{|c|}{ Actual } & \multicolumn{2}{|c|}{ Planned } \\
\hline & 977 & 1978 & 1979 & 1980 & 1981 & 1982 & 1983 & $\underline{198}$ \\
\hline Exploration & & & & & & & & \\
\hline 5 Repo & 146 & 174 & 164 & 147 & 107 & 74 & 63 & \\
\hline Expenditures & 258 & 314 & 316 & 267 & 145 & 71 & 40 & \\
\hline $\operatorname{Mine}(a)$ & & & & & & & & \\
\hline Companies Reporting & 31 & 25 & 26 & 34 & 29 & 23 & 15 & 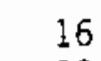 \\
\hline Expenditures & 325 & 271 & 282. & 273 & 212 & 81 & 26 & \\
\hline $\operatorname{Mill}(a)$ & & & & & & & & \\
\hline Companies Rep & 26 & 19 & 26 & 27 & 22 & 15 & 13 & \\
\hline$E$ & 167 & 156 & 203 & $\underline{242}$ & 59 & 11 & 10 & \\
\hline Total Expenditures & 750 & 741 & 801 & 782 & 416 & 163 & 76 & \\
\hline
\end{tabular}

(a) Oate through 1981 are based on editions of Survey of United States Uranium Marketing Activity. Data for 1982 are preliminary figures provided by the Energy Information Administration based on the January 1, 1983, uranium marketing survey.

Source: U.S. DOE. (1983). Table XI-2, p. 64.

economic potential. G. E. Kurt Pralle of Citibank Corporation in New York has outlined three reasons why companies looking to finance new projects will turn to financial institutions (banks) (Nuclear Fuel, 1981a):

- they may have better forms of investments open to them,

- at a particular point in time the company may wish to put more money into dividend payments rather than the project

- the company may feel that with so large an expenditure it is not wise to put retained earnings and share holders funds into a venture.

Pralle discusses that very few innovative methods of financing have been done for uranium mining projects due mainly to uncertainty about ore reserves, operating economics and so forth. The types of financing include:

- balance sheet financing

- project financing

- leverage base method 
- take-or-pay contracts

- production payment method.

Of these, balance sheet financing will form the basis of the majority of loans in the 1980s. Here, money is lent against the balance sheet of the company versus lending to a project with no recourse to any third parties as per the project financing method.

In making loans, the banks look for cost competitiveness of the company rather than relying solely on a sales contract or spot price to ensure them of the project's viability. Banks also look at a company's good credit, sufficient cash flow, project reserves, adequate mining and mill techniques and strong management (Nuclear Fuel, 1981 a and 1981b).

\subsubsection{Financial Data}

The uranium market in 1982 saw a decline in U.S. production, reduced exploration activity and little overall change in the depressed spot market. There has been little to indicate any improvements in the medium-term uranium situation. Under conditions of low market price and steadily rising production costs, many marginally operating mines and operations are being terminated or put on standby. As an example, the number of uranium production sources has fallen steadily since 1979 from a total of 432 to a low of 196 in 1982. The number of underground mine sources has declined from 300 to 139 over the same period (U.S. DOE. 1979, 1980, 1981, 1982, 1983).

Updated financial data is provided for three companies: Homestake Mining Company, Kerr-McGee Corporation and UNC Resources, InC. (Table 4.21). These were chosen because they are representative mine owners/operators in the mine study group as discussed under Section 3.8. Also, these companies breakout the uranium segment in reporting their financial data.

Of the three, only Homestake had an increase in revenues, up $6 \%$ in 1982. Operating earnings were up as well, contributing $40 \%$ of consolidated earnings in 1982 compared to $11 \%$ in 1981. Homestake's capital expenditures dropped dramatically along with a decrease in uranium assets which is indicative of the company's present degree of activity in exploration and development of property. 
TABLE 4.21. Financial Information for Three Uranium-Mining Companies

\begin{tabular}{|c|c|c|c|c|c|c|}
\hline & & Revemues & $\begin{array}{l}\text { Operating } \\
\text { Eamings }\end{array}$ & $\begin{array}{l}\text { Depreciation } \\
\text { and Amortization }\end{array}$ & $\begin{array}{l}\text { Capital } \\
\text { Expenditures }\end{array}$ & Assets \\
\hline \multirow{7}{*}{$\begin{array}{l}\text { Homestake } \\
\text { (Uranium } \\
\text { Division) }\end{array}$} & 1976 & 22,441 & 10,399 & 192 & 2,036 & 14,144 \\
\hline & 1977 & 59,141 & 24,622 & 80 & 2,628 & 45,023 \\
\hline & 1978 & 44,928 & 20,454 & 0 & 7,961 & 42,990 \\
\hline & 1979 & 42,388 & 14,097 & 17 & 8,553 & 47,790 \\
\hline & 1980 & 45,363 & $(601)(a)(b)$ & 6,980 & 12,521 & 54,798 \\
\hline & 1981 & 59,983 & 5,703 & 5,339 & 12,036 (c) & 83,135 \\
\hline & 1982 & 63,702 & 15,592 & 19,953 & 1,025 & 80,831 \\
\hline \multirow{7}{*}{$\begin{array}{l}\text { Kerr-McGee } \\
\text { (Nuclear } \\
\text { Division) }\end{array}$} & 1976 & 96,800 & 32,700 & 7,500 & $\mathrm{NA}$ (d) & 215,300 \\
\hline & 1977 & 123,300 & 22,300 & 9,300 & NA & 236,500 \\
\hline & 1978 & 115,200 & 20,100 & 13,800 & NA & 272,000 \\
\hline & 1979 & 163,400 & $(200)$ & 15,600 & 28,800 & 288,400 \\
\hline & 1980 & 238,900 & 30,000 & 21,300 & 17,900 & 304,800 \\
\hline & 1981 & 201,500 & 26,300 & 16,600 & 14,200 & 304,500 \\
\hline & 1982 & 153,100 & 20,000 & 16,300 & 7,300 & 312,400 \\
\hline \multirow{7}{*}{$\begin{array}{l}\text { UNC } \\
\text { (Uranium } \\
\text { Division) }\end{array}$} & 1976 & 29,339 & 7,103 & 1,070 & 27,856 & 87,222 \\
\hline & 1977 & 80,816 & 28,539 & 1,952 & 54,499 & 145,376 \\
\hline & 1978 & 133,193 & 42,320 & 5,414 & 49,518 & 203,041 \\
\hline & 1979 & 181,626 & 61,339 & 9,677 & 39,156 & 279,436 \\
\hline & 1980 & 167,811 & 12,243 & 11,952 & 46,662 & 239,888 \\
\hline & 1981 & 102,102 & 20,537 & 6,993 & 14,386 & 210,471 \\
\hline & 1982 & 84,038 & 2,409 & 3,249 & 8,606 & 209,791 \\
\hline
\end{tabular}

(a) Includes an $\$ 8,075,000.00$ loss on settlement of uranium litigation.

(b) Value in parenthesis equals loss.

(c) Excludes acquisition of partnership interest.

(d) NA - not available.

Source: Company Annual and Form 10-k reports. 
Kerr-McGee and UNC Resources revenues, operating earnings and capital expenditures were down in 1982 for their nuclear segment. UNC notes that revenues from the sale of uranium concentrate decreased $9 \%$ for 1982 while costs and operating expenses increased 48\%. The company spent $\$ 11.3$ million alone just to place and maintain their uranium mining and milling facilities in a standby condition. 


\subsection{BASELINE FORECAST}

Denand and production forecasts are given below for the years 1983 and 1990. These estimates were derived from a combination of publicly available forecasts. The projections presented here were done in this manner because historically there have been variations between individual forecasts. Taking an average of the various estimates gives a reasonable and representative forecast without having to determine the merit of each individual forecast and its implicit assumptions. In lieu of developing new forecasting mode?s for demand and production a range and average will be given as a representative forecast, as derived from five published forecasts.

\subsection{REACTOR FUEL DEMAND FORECAST}

Demand forecasts from five different sources were used to develop a representative forecast. Reactor demand can be met in three ways: (1) U.S. production, (2) foreign production, and (3) inventory reduction. Consumption and reactor demand are assumed to be equivalent in this section. Table 5.1 shows the demand forecasts, their sources, and the representative forecast.

TABLE 5.1. Annual J.S. Uranium Demand in Tons $\mathrm{U}_{3} \mathrm{O}_{8} \times 10^{3}$

\section{Source}

DOE Grand Junction

Power Magazine (b)

Rocky Mountain Energy

Nuclear Assurance Corporation (d)

Pickard, Lowe, and Garrick (e)

Representative Forecast

Average

Range

\begin{tabular}{|c|c|}
\hline Yea & \\
\hline 1983 & 1990 \\
\hline 16.5 & 23.8 \\
\hline 14.2 & 18.8 \\
\hline 16.3 & 22.5 \\
\hline 16.7 & 20.7 \\
\hline 15.7 & 20.5 \\
\hline 15.9 & 21.3 \\
\hline $14.2-16.7$ & $18.8-23.8$ \\
\hline
\end{tabular}

Sources: (a) DeVergie, 1982. Table 5. (Based on the August 1981 low case prepared by the DOE Office of Uranium Enrichment and Assessment).

(b) Catalano, 1983a. Figure 3.

(c) Lang, 1982. Figure 13.

(d) Leamon, 1982. Figure 5. (U.S. Reasonable Case Demand).

(e) Nuclear Fuei 19836, p. 8. 


\subsection{DEMAND/OTHER USES}

John Hunter, Chief of Reactor Operations, Department of Energy, Washington, D.C., can provide information on uranium demand/use at non-commercial reactors. This information is not immediately available to the public. Since this segment makes up only a small percentage of total uranium demand, it will not be considered here in the ore production forecast.

\subsection{ORE PRODUCTION FORECASTS}

The same five sources that were used in Section 5.1 to develop the reactor fuel demand forecast will be utilized in this section to generate a representative ore production forecast. Explicit forecasts of uranium production were made by two of the sources DOE Grand Junction (DeVergie 1982) and Power (Catalano 1983a). For the other three sources, production forecasts must be derived from the demand forecasts.

Rocky Mountain Energy (Lang 1982) estimated inventory usage in each year of their 1982 to 1996 domestic market demand forecast. Combining their estimated inventory usage with an estimate of imports yields a production forecast as a percentage of demand. For 1983 an estimate of $30 \%$ imports was used. "Presently, about $28 \%$ of all uranium in the U.S. is imported, and this figure is expected to increase" (Catalano, 1983a). For 1990 imports were estimated as $37.5 \%$ of domestic demand which is the proposed import limit. The resulting Rocky Mountain Energy uranium production forecasts as a percentage of demand for 1983 and 1990 are approximately $61 \%$ and 55\%, respectively.

Using the DOE Grand Junction (DeVergie 1982) and Power (Catalano 1983a) forecasts as well as the derived estimate of demand for Rocky Mountain Energy it was determined that U.S. $\mathrm{U}_{3} \mathrm{O}_{8}$ production is an estimated $66.7 \%$ and $54.7 \%$ of reactor demand for the years 1983 and 1990 respectively. Using these values, forecasts of $\mathrm{U}_{3} \mathrm{O}_{8}$ production were made from the Nuclear Assurance Corporation (Leamon 1982) and Pickard, Lowe \& Garrick (Nuclear Fuel 1983b) forecasts of reactor demand. Table 5.2 shows the five $\mathrm{U}_{3} \mathrm{O}_{8}$ production forecasts and the representative forecast. These forecasts consider only uranium demand for commercial reactors, $i . e$. demand for other uses is not included as mentioned in Section 5.2. In Table 5.3 the $\mathrm{U}_{3} \mathrm{O}_{8}$ production is converted to ore production using estimated percent average ore grade as obtained from the U.S. DOE. 
TABLE 5.2. Annual U.S. Uranium Production in Tons $\mathrm{U}_{3} 0_{8} \times 10^{3}$

Source

DOE Group Junction

(a)

Power Magazine

Rocky Mountain Energy $(b, d)$

Nuclear Assurance Corporation $(c, d)$

Pickard, Lowe \& Garrick (c,d)

Representative Forecast

Average

Range

\begin{tabular}{|c|c|}
\hline 1983 & 1990 \\
\hline 10.9 & 9.5 \\
\hline 10.6 & 7.6 \\
\hline 9.9 & 12.4 \\
\hline 11.1 & 9.4 \\
\hline 10.5 & 9.3 \\
\hline 10.6 & 9.6 \\
\hline $9.9-11.1$ & $7.6-12.4$ \\
\hline
\end{tabular}

Unless otherwise noted, references are the same as Table 5.1.

(a) Figure 8. (40 percent import limit).

(b) Assumes 30\% imports for 1983 and 37.5\% for 1990 .

(c) Assumes $66.7 \%$ U.S. reactor demand is supplied by U.S. production for 1983 and $54.7 \%$ for 1990 . (This is the average of the first three estimates).

(d) Estimate is derived from U.S. reactor demand forecast.

\section{TABLE 5.3. Annual Ore Production}

\begin{tabular}{|c|c|c|c|}
\hline (Year) & (Tons $\mathrm{U}_{3} \mathrm{O}_{8}$ ) & $\begin{array}{l}\text { Average Ore } \\
\text { Grade, } \%(a)\end{array}$ & (Tons 0re) \\
\hline 1983 & 10,600 & 0.12 & $8,833,333$ \\
\hline 1990 & 9,600 & 0.13 & $7,384,615$ \\
\hline
\end{tabular}

(a) Conventionally mined and milled ore only. Information on percent average ore grade obtained from the DOE, Grand Junction Area office, Grand Junction, Colorado. 
.

( 


\subsection{RADIONUCLIDE EMISSIONS TO AIR}

This section will develop several important aspects of radionuclide emissions to air beginning with a discussion of the parameters and details involved in developing a model for emission measurement. This will be followed by a detailed description of three methods to measure/monitor radionuclide emissions followed by special topics on instrument calibration and vent flow measurement.

As an alternative method for reducing radionuclide emissions, increasing the effective height of release has been considered. A discussion of the increased cost to production resulting from the installization of a 20 -meterhigh vent stack will be given.

This section will then conclude with a discussion of the impact that current and projected mining activity (ore production) will have on the magnitude of radionuclide emissions to air.

6.1 TECHNIQUES FOR MONITORING ${ }^{222}$ Rn EMISSIONS FROM UNOERGROUNO URANIUM MINES

The determination of ${ }^{222} \mathrm{Rn}$ emissions in uranium mine ventilation exhausts can be reliably made by direct field measurements of radon concentration in the air exhausted from the vent and the vent air-flow rate. It is impractical to rely on measurements of the concentrations of radon or its short-lived daughters taken underground to determine radon emission rates because the underground measurements are normally taken only in areas where active mine work is in progress. Those areas usually receive the main flow of ventilation air and therefore, with the exception of lunch and shop areas have some of the lowest radon and radon daughter concentrations in the mine. However, a large portion of the radon emanating surfaces of most mines consist of old mined-out areas. Those areas are often bulkheaded (closed off) from the active areas of the mine and receive only enough ventilation to keep them at a negative pressure relative to the active areas. Since a large part of the inactive area is often inaccessible, there will be no radon or radon daughter measurements available from them. The air pumped from those areas to the surface tends to have relatively high $222 \mathrm{Rn}$ concentrations but low flow rates and usually contains a large fraction of the total emission of radon. 
A model for predicting future vent emissions from past radon emission rate measurements or estimating them from other kinds of measurements, has not been completed. Jackson et a1. (1979 and 1980) found that on average, about 50\% of the variation of the radon emission from uranium mine vents could be related to the cumulative production of the mine (tons of ore) since it was first opened. This correlation was predicted on the theory that a mine expands in volume during its life, creating additional radon emanating surfaces. The radon emanation from the surfaces containing exposed ore should be greater per unit area than that from worked-out surfaces where the ore has been extracted. However, there is always a residual quantity of uranium minerals in the workedout surfaces since there is a limit to the ore grade which can economically be mined. As the mine ages, the worked-out areas continue to expand while the surfaces being mined at any given time remain relatively constant. Thus, eventually the worked-out areas grow so large that the emanated radon from those areas exceeds that from exposed ore and the radon emanation will increase with some functional relationship to the total amount of ore extracted. If the mine is considered to be a lengthening tube of constant cross section, then a linear relationship is predicted.

This partial model does not consider several factors which can be extremely important in making predictions for individual mines or vents. Perhaps the most important of these is the stability of the rock which is being mined. After a section of a mine has been abandoned, if the rock is unstable there will be many rock falls and cave-ins after timbers rot and pillars have been removed. Whether these cave-ins result in a greater or lesser amount of radon emanation will depend on whether there is a greatly increased surface area exposed or whether that area of the mine becomes totally sealed from ventilation to the outdoors. Other important factors that could modify the model are the degree to which water is transporting radon to the various surfaces, and the average porosity and permeability of the rock structures in individual mines or ventilated areas.

Thus, while it may be possible to relate radon emissions to production on an industry-wide average, it is not reliable to use this existing partial model 
to predict emissions from a specific mine for which no measurements are available. The accuracy of the model for extrapolating to future emissions from mines where measurements have been taken in the past, will depend on how closely its assumptions fit the practices in individual cases, and on the perturbations from rock fall already mentioned.

For the purposes of other sections of this report, the partial model will be used to predict increases from previous mine measurements as the mine ages. For those mines which have been reported to be totally shut down with the vents sealed, it will be assumed that there are no current or future emissions. For mines where only a small fraction of the mine near the shaft is occasionally ventilated to permit access to water pumps and the rest of the mine is sealed and bulkheaded from the ventilated area, no calculations will be made. Emissions from such mines may be substantial for short intervals of time when vent fans are turned on, but the average emissions should be only a small fraction of the total measured previously for the entire mine. In this report, these mines will be classified as sealed. Based on these assumptions, the effect of mine shut down and capping will be to totally remove emissions from a fraction of the mines where measurements have been taken. Conversely, increases in production will increase emissions at the remaining mines.

\section{1 .1 Radon Measurements}

There are three potential approaches for monitoring the quantity of ${ }^{222_{R n}}$ emitted from uranium mine vents in a selected time interval. In each of these approaches, one measures an average concentration of $222_{R n}$ which must be multiplied by the measured air-flow rate of the vent in order to estimate the quantity per unit time. The three radon measurement techniques are: grab sampling, continuous sequential sampling, and integrating sampling. Certain elements of these sampling techniques are characteristic of stack monitoring for any species and not merely for radon or other radionuclides.

Grab sampling is, as the name implies, the collection of a sample of air from the stack during a single, short period of time or several samples during sequential, short periods of time. It is simple, direct, and normally low cost 
in terms of equipment. It does, however, pose problems as to the representativeness of the sample(s), and tends to be a labor-intensive technique in terms of manhours per data point.

Continuous sequential sampling is typified by an instrument that is installed at the stack to continuously draw a fraction of the exhaust air and pass the air to an analyzer. The analyzer monitors the concentration of some constituent, records the measurement at intervals, and resets itself to collect and record a new measurement. The primary use of this type of instrumentation is to monitor rapidly changing concentrations. Continuous samplers can establish their time dependency and the range of concentrations which may be encountered when grab sampling. Of course, data taken over an extended interval(s) can also be averaged. Since the instruments can give enough measurements to establish both the average and the dispersion of concentrations, they are usefut for research applications. The instruments can provide for continuous monitoring of stacks where grab sampling is not satisfactory because of large and unpredictable fluctuations in concentrations.

One drawback of the automatic sequential sampling method is that the equipment is complex and costly. Calibrations and quality assurance procedures for this technique generally require more careful testing of secondary effects on the efficiency from such characteristics of the instrument as the rate of flow, pressure differentials, and air leaks. Other characteristics that can affect measurements are the instrument's sensitivity to ambient temperature and to constituents of the ambient air such as moisture, and its response to particulates in the airstreams. This type of instrument can be susceptible to damage in hostile environments. Calibrations can change with time, necessitating periodic maintenance and frequent operating checks. Costs can be substantial, but since large amounts of data are collected, normally the unit cost per data point is small.

Integrating samplers continuously collect a portion of the total stack flow or just a portion of the constituent being measured over an extended interval. Then the samplers are normally removed from the stack and the total content of the desired constituent is measured. These devices are useful for 
determining the average concentration with a sampling interval which may range from hours or days up to a year or more depending on the individual instrument.

Some integrating samplers are designed to function without moving parts. For example, the species of interest may enter an evacuated chamber through a small orifice or a particulate species may be collected electrostatically as long as a sufficient electrostatic field can be maintained. Radionuclide exposure rates can be integrated from the darkening of tracks or the increasing number of tracks created in radiation sensitive materials. An example of a mechanical integrator is an air filter that can sample continuously until the particulate loading causes a significant loss of flow rate which causes the sampling to cease. Non-mechanical integrating samplers are often simple in design and low in unit cost. These non-mechanical types are useful when large numbers of long-term measurements are desired. However, only a great deal of study can establish that there is a consistent response-to-concentration relationship for integrators. Nonlinear response characteristics, if present, can mean that the average recorded for the sampled interval is incorrect when a period of high concentration and one of low concentration occurs in that interval. Effects from detector saturation, fading of the response characteristic, background noise variation during long intervals, and changes in the flow rate through the detector as well as the stack must be considered and if necessary, compensated for. Sensitivity of the detector to other constituents which might be present must also be considered. While many of these problems are not unique to integrators, the nature of the measurement techniques can make it difficult to detect such problems from an examination of the data. However, when properly applied, these integrators can provide a large number of economical long-term measurements. The application of these three approaches to radon measurement and some of the equipment typically used will be covered in the next sections.

Grab Sampling and Radon Measurement

The most common technique for ${ }^{22}$ Rn measurement is the scintillation flask grab sample (device manufactured by companies No. $2,4,5,6$ listed in Table 6.1). In this technique, one uses a flask which has been internally coated with a scintillator, such as zinc sulfide. It is filled with the 
TABLE 6.1. Manufacturers of Radon Analysis Equipment

1. Aero Vironment

145 Vista Avenue

Pasadena, California

2. Eberiine Instrument Corporation

P.0. Box 2108

Santa Fe, New Mexico 87501

3. EDA Instruments, Incorporated

5151 Ward Road

Wheat Ridge, Colorado 80033

4. Johnston Laboratories, Incorporated

3 Industry Lane

Corkeysville, Maryland 21030

5. Pylon Electronic Development Company Ltd.

47 Colonade Road

Ottawa, Canada KZE 729

6. Rocky Mountain Scientific Glassblowing Company

2520 Galena

Aurora, Colorado 20010

7. Terradex Incorporated

460 North wiget Lane

Walnut Creek, California 94598

atmosphere to be sampled, either by pumping air through a flask designed for flow-through operation or by evacuating a flask and allowing the air being sampled to fill the flask.

In the air filling process, the air is usually filtered at the inlet to establish a point in time when there are no radon progeny present in the flask and to prevent the contamination of its interior with long-lived alpha particle enitting radionuclides. The inlet air for the flask is collected from either an access port in the vent or from a point in the mouth of the vent that has been determined to have a uniform outward air flow pattern. If large quantities of water droplets are present, an inline trap should be used to prevent the entry of water into the inlet filter.

After waiting for about four to five hours to permit ingrowing radon progeny to approach equilibrium with the radon, the scintillation flask is placed 
on a bare photomultiplier tube. This tube can detect scintillations coming from the interaction of alpha particles with the interior coating. The photomultiplier tube is housed in a light-tight container. To prevent damage to the tube, its operating bias voltage is removed whenever the container is opened. It is good practice to place the scintillation flask in the light-tight container and close it for at least five minutes prior to counting to allow the decay of phosphorescence that is induced in the scintillator and photomultiplier tube during exposure to light. After the delay, the scintillations are integrated for an interval that depends on the radon concentration and the sensitivity and precision desired. For monitoring uranium mine stack concentrations, ten minutes to one hour are usually sufficient to permit a precise measurement. Following the count, the air is evacuated from the flask and the flask is rinsed several times with filtered ambient outdoor air. The flask can be stored either evacuated or filled with filtered outdoor air. After a delay of six or more hours, the flask can be returned to the counting chamber to permit determination of its background for subsequent counts.

It is typical to encounter a memory effect when plastic flasks are used, from the finite solubility of radon in plastic materials. This effect was reported by Jackson et al. (1979) to be about $1 / 2$ to $1 \%$ of the previous count. It would be expected to diminish with a half life somewhat shorter than the radioactive half-life of radon because of outgasing. Buildup of static charges on the surfaces of the flask during handling can cause serious measurement errors when using nonmetallic flasks in dry atmospheres. The buildup of charge can influence the location of deposited short-lived radon progeny in the flask and thus the detection efficiency. Care should be used when handling flasks not to wipe their surfaces. Comnercial antistatic sprays are helpful, but should be applied at least 4 to 5 hours before making a measurement to allow the decay of previously deposited radon progeny.

As noted previously, results based on grab samples can be difficult to defend if there is no evidence showing the dispersion or range of results that can be expected in a given sampling situation. In particular, radon concentrations in mine air have been shown to have a diurnal cycle caused in turn by the diurnal cycle of the air density. Smaller variations may be caused by the air 
temperature and from the effects on the underground air density produced by winds blowing across or into portals and vents. In addition, regular mining operations and conditions such as the position of the elevators in shafts or blasting could produce short-term changes in the radon concentration. Profound effects can be expected from fan shutdowns or changes in the underground ventilation air path. Thus, it is always necessary to use a sampling protocol which establishes the dispersion of measurements when grab sampling.

As minimum, duplicate samples collected on two different days at different times of the day can give information about the magnitude of cyclic variations versus replication errors. However, even when this type of sampling is used, it is best to avoid the times of blasting or times when the ventilation of the mine can be expected to be abnormal, if one is attempting to obtain average emission rates from a few measurements. Of course, the abnormal conditions could cause such profound radon concentration changes that they would significantly affect the average. This minimum protocol was followed by Jackson et a1. (1980) and was evaluated using two systems of continuous sequential monitors and a statistical evaluation of the dispersion of the measurements. While that interpretation indicated that the relative standard deviation of measurements involved in counting scintillation flasks was about $4 \%$ when four samples were averaged, the sampling errors appeared to be larger. The ratio of the maximum observed result to the average result measured hourly for about one week periods at three vents ranged from 1.13 to 2.0 . At a fourth vent, variations caused by cyclic vent shutdowns produced ratios as high as 7.3 . While Jackson et a1. (1980) determined that these variations tended to produce a relatively small error in their measurement of the annual average radon emission from the entire U.S. mining industry, based on grab sample determinations of radon in exhaust air, their effect on the average emission rate determined for a single mine can be substantial. Where possible, a large number of grab samples should be collected so that patterns of emission rates and correlations with operations can he established. However, if a very detailed and extensive study at a few vents is to be performed, the cost of grab sampling could be prohibitive and some form of continuous sequential monitoring should be considered. 
When grab sampling, the use of replicates taken at different times, days of the week (operating and holidays), and taken over a substantial portion of the year and analyzed with a statistical technique known as the analysis of variance can yield information about the long- and short-term concentration changes that can be expected. The data-handling methods and the design of experiments used with this technique are covered in standard statistical texts and will not be repeated here. As a basis for estimating the average emissions from any mine vent using grab sampling, the following protocol is suggested.

1. On a work day morning, at least one hour after shift change and blasting, collect a duplicate set of grab samples within a fiveminute interval and measure the vent flowrate.

2. Collect a second duplicate set of grab samples on the afternoon of the same day or within a few days of the original set (sample on working days only) and remeasure the vent flowrate. The afternoon sample should be collected at least one hour after lunch break and/or blasting.

3. Collect a set of two samples, one 5 to 15 minutes after blasting and the other 40 to 60 minutes after blasting. These samples will give information about the concentration maximum, and the rate of its decrease following a blast.

4. Collect a set of samples on a weekend or at least two hours after the last working shift on a weekday. (If ventilation changes have been made, see Step 5 below.)

5. If any shutdown of vent hole ventilation fans or if a flow reversal is a normal procedure at the mine, sample all of the vents at the mine within the intervals from 30 to 60 minutes before returning the ventilation fan(s) to normal status, from 10 to 30 minutes after returning the ventilation to normal, and again in the interval from 60 to 120 minutes after the ventilation fans have returned to normal operation. Measure the vent flow rates within 30 minutes of each sampling (with the fans on and with the fans off). Indicate the directions of flow as necessary. 
6. Repeat Steps 1 or 2 on quarterly intervals for one year.

This protocol will require at least 14 grab samples per vent, collected over a one-year interval. It should be capable of accurately determining the annual average emission rate of ${ }^{222} \mathrm{Rn}$ for each mine vent and estimating the precision of the measurements. It should give enough information about the effect of operations on the radon emission rates to determine if they are significant enough to require additional study. Since measurements are continued for one year, relationships of radon emission rates to continuing production and excavation should be established.

Continuous Sequential Sampling for the Measurement of $222 \mathrm{Rn}$

There are two and possibly three commercially available continuous sequential sampler designs that could potentially be used for measuring radon in mine ventilation air. The first is an automated flow-through scintillation flask. The Eberline RGM II is an example manufactured by company No. 2 listed in Table 6.1. The Eberline instrument uses a precalibrated microprocessor to compute and print results in units of $\mathrm{pCi} / 2$. This type of device draws fittered air continuously through a scintillation flask, stores scintillations detected by its photo-multiplier tube for an interval, computes the result, clears its scaler, and accumulates new counts at the preset intervals. The 1983 price for this unit is approximately $\$ 7800$. Since the ingrowing radon daughters accumulate on the walls of the detector, their alpha particle emissions contribute to the count from radon in the air and they are included in the calibration. Even when using short counting intervals, it is not possibie for this type of counter to accurately follow radon concentrations that are changing rapidly relative to the 2 to 3 hours time required for the equilibration of ingrowing daughters. However, the counts accumulated in later intervals will tend to compensate for short-term errors in earlier intervals so that this instrument can provide unbiased estimates of averaged concentrations for periods of about a day or more while giving approximations to the more rapidly changing concentrations. A deconvolution routine was reported (Thomas 1972) to establish the correct instantaneous concentrations using shorter counting intervals even when they are rapidiy changing. However, the calibrations required for that routine are difficult and that type of routine can still 
produce significant aberrations in the results for concentrations which are changing significantly within the counting intervals rather than just between intervais (Busigin, Van der Vooren and Phillips 1979).

The Eberline instrument is designed to be used in field applications and is equipped with a weather-resistant cover. It weighs about 110 pounds and is not readily portable. These samplers count radiations at the time the sample is passing through the flask and are sensitive to the alpha particles emitted by ${ }^{220} \mathrm{Rn}$ (thoron) and its short-lived daughters. Because of ${ }^{220} \mathrm{Rn}$ 's 56 -second half life, it is possible to eliminate ${ }^{220} \mathrm{Rn}$ from the air stream by the use of a charcoal delay bed (not normally supplied with the instrument). Saturation of the charcoal bed should be designed to occur after an interval of about five minutes with subsequent elusion of the radon from the bed. After a period of about 15 minutes of operation, such a delay bed achieves a steady state with its characteristic hold-up time. Since the ${ }^{220} \mathrm{Rn}$ in sampled air is unsupported by its longer-lived parents, this delay would be sufficient to remove about $95 \%$ of the thoron from the air stream. It would produce a small delay in the response of the instrument to changing ${ }^{222}$ Rn concentrations, but for practical purposes the delay is insignificant.

When installing this or other types of continuous samplers, consideration must be given to the moisture and particulate content of the inlet air. There is occasionally a considerable quantity of water exhausted from uranium mine vents when the shaft penetrates an aquifer. Since this water is usually in the form of coarse droplets, it can be removed by using a trap. For extremely large volumes of water, it will be necessary to use a self draining trap with water seals. Because of the limited solubility of radon in water and the relatively small water-to-air ratio, this type of trap should not significantly reduce the radon concentration in the air being sampled. If dust loading is a significant problem, a larger prefilter than is normally supplied with the Eberline unit should be considered.

The RGA 400 radon gas analyzer is a second type of commercially available continuous sequential radon gas analyzer (manufactured by company No. 3 listed in Table 6.1). This analyzer uses electrostatic precipitation to collect the 
daughters of ${ }^{222} \mathrm{Rn}$ and ${ }^{220} \mathrm{Rn}$ onto the surface of a surface barrier diode detector where their alpha particle emissions can be measured spectroscopically. In this instrument, the radon and thoron progeny consist largely of ions generated in the prefiltered air sample because of radioactive decay. In principal, these ions can be concentrated at the detector. The unit features a programmable microprocessor and is protected from accidental power failure by a battery backup which insures up to ten hours of operation with no data loss in the event of a power failure. This unit also has provision for measurement of the accumulated radon and thoron progeny on the inlet filter so that it can provide a sequential series of measurements of those radionuclides in addition to the radon and thoron. The 1983 price for this unit is approximately $\$ 16,900$. Since the RGA 400 is a relatively new design, there is very little information available to date concerning its applicability to stack sampling. The electrostatic field collector should be sensitive to the moisture of the sampled air. However, it is our understanding that internal calibrations for the relative humidity are provided in the microprocessor program. The same type of prefiltering and water removal used for the flow-through flask sampler may also be necessary for the RGA sampler. If these preseparators are used, radon progeny measurements will be meaningless, however, and only their radon and thoron measurements can be used.

A third type of continuous sequential sampler that could potentially be modified for stack sampling is a sequential bag sampler. In this design, air is pumped sequentially into an array of bags at a low flow rate. One such unit of the AQS series of samplers of the Aero Vironment Company utilizes Tedlar(a) bags which are reported by Jackson et al. (1979) to be satisfactory for retaining radon in air samples (manufactured by company No. 1 1isted in Table 6.1).

The AQS is not designed as a stack sampler. It is housed in a large barrel with ambient air being collected from a covered ported area at the top of the barrel. The air is then transferred into bags within the barrel using a small pump for each bag. Each pump operated in a programmed sequence. However, Jackson et al. (1979) modified these samplers by attaching an inlet vent

(a) Tedlaro is a trademark of the Dupont Company. 
and filtration system between the vent and the instrument. As long as the vent operated, the air velocity was sufficient to rapidly force air through the vent and into the sampled cavity. A sequence of up to twelve bags could be used per 24-hour sampling. Once each day the filled bags were removed and replaced with einpty bags and the sequence was repeated. An aliquot of the air from each bag was transferred into an evacuated scintillation flask to permit analysis of the radon content of the sampled air. Thus, this technique could be considered to be sequential or integral sampling coupled with scintillation flask counting. Since Tedlar bags are rather brittle, it is necessary to be aware of small cracks and leaks that can develop in handing. Bags have a rather limited useful life because they are being regularly evacuated and filled causing wrinkling and ultimately cracking. This type of sequential sampler can be programmed to detect concentration variations in various time intervals depending on the number of bags and switching arrangement that is chosen. Integrating Radon Samplers

The two principal types of integrating radon samplers that are commercially available are the "Track Etcho"(a) filter cups marketed by Terradex Corporation (manufactured by company No. 7 listed in Table 6.1) and the passive environmental radon monitors (PERMS) marketed by a number of firms. PERMS are designed for environmental use but they are not readily suitable for in-stack applications because of their size and mode of operation. One commercially available PERM is the RDT-310 available from EDA Instruments, Inc. (manufactured by company No. 3 listed in Table 6.1 ). The unit is a cylinder about $10 \mathrm{in}$. in diameter and $15 \mathrm{in.}$ in height and contains batteries to permit electrostatic collection of radon progeny at a point near a thermoluminescent chip which is sensitive to alpha particles. These units rely on the passage of air through the housing, then through either a drying agent or a semi-permeable membrane which passes radon but not moisture into a cavity where the radon progeny are collected from the air. These units could possibly be used for stack sampling at some sites if mounted in a separate housing that is ducted to the vent stack. However, this application would significantly increase the cost of the installation.

(a) Track Etch ${ }^{\circ}$ is a trademark of the Terradex Corporation. 
The Track Etch cup is a simple apparatus which uses (for a radon detector) a film chip that forms detectable tracks by degradation of the chip material along the path of alpha particles. The chip is mounted inside of a plastic cup which has a plastic-coated filter material across its open end. The filter material allows the passage of radon but not its daughters or other particulates into the cup. After several hours, the radon in the cup reaches radioactive equilibrium with its daughters. A portion of the alpha emitting radionuclides in the cup produces tracks in the detector film. After a period of exposure, the cups must be returned to the manufacturer where the detector films are developed by etching to enlarge the tracks. The tracks per unit area are then measured using microscopes and converted to radon concentrations.

These cups have been used extensively for environmental monitoring and exploration work in the uranium industry. The use of the Track Etch cup in a vent stack would require some precautions in mounting because of the highvelocity air. Special sealing of the filter cap and protection from physical stress would be needed. In addition, since the cup and its cover are constructed of plastic, tests should be performed to assure that the buildup of electrostatic charge from the air stream onto the surfaces does not effect the calibration.

Since units are relatively sensitive, the length of exposure would have to be adjusted to be certain the number of tracks does not exceed the upper limit of readability. Because of the finite time required to reach equilibrium between the internal and external atmospheres, these devices should require at least several days of exposure to conform to the original calibration. It is possible that special calibrations might be necessary for exposures in those vents with extremely high radon concentrations. The use of several grab scintillation flask samples might be more practical for vents emitting extremely. high concentrations. For lower concentrations, where standard calibrations can be used, this technique offers the potential of long-term integrated measurements at a number of sampling sites. This can be especially useful when a large-scale program is being considered.

There are no reports addressing the application of Track Etch ${ }^{2}$ to vent stack sampling, and the available reports primarily discuss experiences in 
home-radon monitoring. Bruno, Israeli and Magno (1983) report that the relative standard deviation of this technique for replicate $1 / 2$ to one-year long measurements in homes in Butte, Montana, is about 15 to $16 \%$. However, they report that when Track Etch ${ }^{*}$, PERMS, and Eberline continuous sequential radon monitors were compared, the $95 \%$ confidence interval of the ratios of simultaneous measurements for any pair of instruments was about $+/-70 \%$. To assure that results are accurate, stack monitoring programs using Track Etch cups should include at least a few cross checks with another accepted monitoring technique such as multiple grab scintillation flask samples. Replicate analyses should be made at enough vents to determine precision of the technique for that application.

\subsubsection{Calibration of Radon Monitoring Instruments}

There are presently no nationally- or internationally-recognized standard radon sources. For this reason, major laboratories involved in radon measurements have (for the last two years) been participating in a series of roundrobin intercalibration studies. The large-volume test chamber at the Environmental Measurements Laboratory of the Department of Energy has been used to provide a source of air containing moderate levels of ${ }^{22} 2_{R n}$ so that flask grab samplers could be compared. In addition, a special pulse ionization chamber flask has been designed at that laboratory to have theoretically predictable counting efficiency characteristics (a defined, small, dead volume for the flask). On the basis that measurements taken using that flask are accurate, other laboratories have been correcting the efficiencies of their instruments to remove the bias between lahoratories (Fisenne, George and Keller 1983). While there are a number of other comercially available radon sources or calibration techniques, calibrations based on them must be considered tentative until a recognized national standard for radon measurement has been established.

At present, two national laboratories have controlled atmosphere test chambers which are being calibrated in relationship to the pulse ionization chamber flask. The Environmental Measurements Laboratory of the U.S. Department of Energy has been selected to provide calibrated test atmospheres which contain ${ }^{22} 2_{R n}$ at environinental levels for calibrating instruments upon 
request. The U.S. Bureau of Mines, Denver Research Center has been selected to provide the same service with ${ }^{222}$ Rn concentrations at the occupational levels encountered in uranium mining. These chambers are designed for relatively short-term exposures for a number of users. Long-term exposure calibrations for instruments may need to be done by making a series of short-term measurements at a field site using a calibrated short term type of instrument during an interval that is monitored by the long-term samplers. Then the long-term measurements could be related to the average of the calibrated shorter-term series of measurements.

\subsubsection{Vent flow Measurements}

To determine the radon emission rate for a vent, the air-flow rate must also be measured. Depending on the face velocity of the air (and other factors), any one of a number of the instruments which are used to monitor highvelocity air in ducts may be used. These include pitot tubes, vane anemometers, hot-wire anemometers and the more costly instruments such as those which make measurements based on the bending of electrical corona discharge, sonar, and the change of electrical capacitance.

In most cases, the field conditions for measurements will be less satisfactory than the ideal conditions which are specified for these instruments, and compromises will be necessary. For example, it will not be possible to use hot-wire anemometers at vents that are emitting a water spray because the readings will be affected by the heat capacity and evaporation of the water. Pitot tubes are not suitable for use unless they can be inserted into the duct. Even then, it is commonly specified that there should be at least ten duct diameters of straight duct upstream and downstream from the neasurement point for pitot tube measurements. There are rarely more than five duct diameters of uranium mine vents above the ground. Above-ground sections contain the exhaust fan and flow-straightening fins in close proximity to the measurement point. At some vents, no access holes are available to insert a pitot tube and some type of measurement will have to be taken at the mouth of the vent. One accurate technique would be to measure the time it takes for a tracer injected at the base of a vent to reach the surface if the exact length of the vent hole were known. However, this technique cannot be universally applied because the bases 
of a number of vents will be in areas bulkheaded to prevent access because of high radon-progeny concentrations and/or the danger of falling rock.

Thus, there are a variety of conditions to cope with when determining the flow rate. The effect of those conditions on each measurement will have to be considered based on past experience or will have to be determined by cross checks between instruments. Some of the more costly techniques may be less susceptible to difficulties from ambient conditions but we have not tested them. Jackson et al. (1980) made some cross checks with vane anemometers and pitot tubes and cross checks between different pitot tubes at different times. Most of the average measurements agreed to within a range of about $20 \%$. There are often severe flow discontinuities in these vent stacks, so that it is typical to make numerous measurements at area increments representative of the cross section and average them. In addition, Jackson et al. (1980) concluded from measurement at two mine vents that temporal variations of the vent flow were relatively small. However, the universal applicability of this limited test was not demonstrated, and it would be best to measure the flow within as short of time as possible, of collecting air samples when making radon measurements.

\subsection{EMISSIONS CONTROL DESCRIPTIDN}

The Environmental Protection Agency has considered several options by which their proposed national standard for radionuclide emissions $\left({ }^{222} \mathrm{Rn}\right)$ from underground uranium mines could be met. (a) The EPA noted that the development of such an emission standard for underground uranium mines was more difficult and complicated than for other sources emitting radionuclides into air (Federal Register, 1983).

One reason for this difficulty is because there are no ${ }^{22} 2_{\text {Rn emissions }}$ source control systems presently in use in underground uranium mines upon which to gage a standard, and many proposed control systems were found to be too

\footnotetext{
(a) The EPA standard limits the annual average ${ }^{222}$ Rn concentration in air due to emissions from underground mines to $0.2 \mathrm{pCi} / \ell$ above background in any inrestricted area.
} 
costly and not very effective. The EPA thus concluded that there is no existing practical source control technology for achieving acceptable reductions in ${ }^{222} \mathrm{Rn}$ emissions to air from underground uranium mines.

As alternative control methods, the EPA has suggested the standard be met by any of the following procedures: 1) reducing the percentage of time the mine operates, 2) controlling additional land around the mine site, (a) or 3) increasing the effective height of the release (Federal Register, 1983). The EPA has suggested that perhaps the most effective procedure for 1 imiting ${ }^{222} \mathrm{Rn}$ exposure to individuals might be to provide for a greater dispersion of the released ${ }^{222} \mathrm{Rn}$ from the mine. That is, as per number 3 above, increasing the vent stack height (Federal Register, 1983). Under request by the EPA, the Pacific Northwest Laboratory has estimated the cost of a 20 meter high exhaust borehole vent stack and determined the levelized cost of such vent stacks per ton of ore and per pound of $\mathrm{U}_{3} \mathrm{O}_{8}$ for a best, worst, and most likely case.

\section{2 .1 Stacks}

Data used in the estimation of the exhaust borehole vent stack cost was obtained from vendors and cost estimating manuals (Means 1982, Engelsman 1981, Page 1977). All necessary equipment was assumed to be rented. Labor rates include: fringe benefits, insurance, taxes, and contractors' overheads and profits. Final dollar values are given in January 1983 dollars. Price data from Engelsman (1981) were escalated up to January, 1982 dollars by increasing the values $6.7 \%$ in accord with the Marshall and Swift quarterly equipment cost index for mining and milling (Chemical Engineering 1981, 1982). These price data were then escalated to January 1983 dollars by assuming a $5 \%$ increase during 1982 .

In estimating the cost it was originaliy assumed that the stack would be 20 meters high and 4.5 feet in diameter. However, upon further investigation it was found that the most common size exhaust borehole has a diameter of 6.0 feet. Thus, the estimates were scaled up to this size.

(a) Land control cost out to $2 \mathrm{~km}$ is discussed in Section 3.8.2, Land Valuation. 
Foundation loading was designed to be 850 pounds per square foot. This value is much lower than it normally would need to be. The reason for using such a conservative value is because the soil type and seismic zone are not known. Also, using a low value for foundation loading allows the weight of the stack to be distributed around the cased boreholes. Thus, no engineering or other changes would need to be made to the boreholes.

Wind loading of the stack was also calculated. A value of 20 pounds per square foot was used to determine the load and a safety factor of $67 \%$ was added. It was found that the most economical method of support would be the use of guy wires and a quote was obtained for this material.

The major cost component in the estimate is the material of which the stack is made. Prices for two different kinds of stacks were obtained. As mentioned before, the original design has a diameter of 4.5 feet. A quote for this size stack was obtained from Seattle Boiler Works. This design called for the stack to be made of $3 / 8$ inch carbon plate steel. It would be made in two sections (for easy transport) with a flange in the middle and at the bottom. $A$ reinforcing ring two-thirds of the way up was included for attachment of the guy wires. The alternative design was made out of 10 gauge corrugated galvanized spiral steel and prices were obtained from Engelsman (1981).

When scaling up to 6.0 feet it was estimated that the total structure cost would increase in the same proportion as the stack material costs. The cost of the carbon plate steel stack increases linearly with the diameter, thus the price was increased $33 \%$. For the corrugated steel structure it was found that the thickness of the metal would have to be increased to 8 gauge steel and the price would increase $50 \%$. The final cost of the carbon plate steel stack was $\$ 16,550$ and the cost of the corrugated steel stack was found to be $\$ 13,030$. A detailed cost estimate appears in Table 6.2.

A11 information about size, required number of exhaust boreholes, and average mine 1 ifetime production were derived from data for three typical mine types. Specifically, production ranged from 330 to 700 tons per day, and the number of stacks required (of various sizes) was 2 to 13 . The years of mine production remaining spanned 2 to 12 years and the average ore grade over the 
IABLE 6.2. Exhaust Borehole Stack Cost Final Estimate

Exhaust Borehole Stack Cost

Final Estimate

\section{Excavation \\ Backhoe w/operator (1/2 Day) \\ Operating cost $\$ 5.70$ hour}

Foundation

$6^{\prime} \times 6^{\prime}$ concrete reinforced

with anchor bolts

Stack

Material

Labor to assemble

Painting

Crane w/operator (for assembly

and erection)

55 ton (truck MTD.) hydraulic

(a) $\$ 855 /$ day

Operating cost $\$ 16.35 / \mathrm{hr}$

Guy Wires

Cable (coated)

Anchor plates, tie rods, and

turnbuckles

Clamps

Erection Labor

3 struct. steel workers $\$ 25.50 / \mathrm{hr}$

1 struct. steel foreman a $\$ 28.15 / \mathrm{hr}$

1 surveyor w/transit for 2 hours

Backfill and Compact

I Ramer compactor (gas)

Operating Cost $\$ 0.88 / \mathrm{hr}$

1 Laborer a $\$ 18.70 / \mathrm{hr}$

Convert ' 82 to ' 83 dollars (5\%)

Scale up to $6^{\prime}$

(most bore holes are 6' dia.)

\section{Corrugated Steel}

Galvanized Spiral

$\$ 215.00$

25.00

320.00

$4,430.00$
360.00

$1,285.00$

200.00

855.00

130.00

65.00

65.00

125.00

80.00

125.00

80.00

615.00

225.00

80.00

615.00

225.00

80.00

45.00

10.00

45.00

190.00

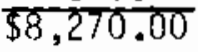

10.00

190.00

$\$ 12,245.00$

$\$ 8,685.00$

$\$ 12,410.00$

$\sim 50 \%$

(8 vs. 10 gauge)

$\$ 13,030.00$ $\sim 33 \%$

(5tiil 3/8")

$\$ 16,550.00$ 
same period varied between $0.15 \%$ and $0.40 \% \mathrm{U}_{3} \mathrm{O}_{8}$. A financial analysis was performed to determine the levelized cost of installing the stacks on all exhaust boreholes of a diameter greater than one foot. Development of the financial analys is methodology is included at the end of the report as Appendix $G$.

Certain costs that are location and/or operation dependent were not included in the basic stack cost estimate. However, these costs are included in the financial analysis and appear below:

- Mobilization and Demobilization of Equipment: This cost would depend on the distance of the mine from available rental equipment, ease of access, and the number of stacks being constructed. Including these costs could easily double the equipment costs and increase the stack cost by 10 to 20 percent.

- Transportation of Labor or Material to and from the Mine: As with the equipment cost, labor costs could be double when including transportation to and from the mine. This would also result in an increased stack cost of 10 to 20 percent. Transportation of materials to the mine could be extremely expensive if it must be fabricated some distance away. For example, the plate steel stack may have to be made hundreds of miles from the mine, which could significantly increase cost.

- Increased Ventilation Cost: This cost would depend for the most part on the length of the borehole. Assuming that the fans could handle the additional load, the only cost would be the increased power consumption. With current prices and assuming $80,000 \mathrm{cfm}$, increased power consumption of $10 \%$ would cost about $\$ 4,000$ a year per exhaust borehole stack. Under some conditions requiring additional capital investment in the ventilation system, the cost could be considerably greater.

- Prevention of Stack Icing: To prevent icing of the stack during cold weather it would be necessary to either heat the stack or heat the air before it goes into the stack. Assuming it would be necessary to raise the temperature of the exhaust air $10^{\circ} \mathrm{F}$ and the air 
flow was $80,000 \mathrm{cfm}$, a heater would be required to supply about 24 million Btu per day. Such a heater is available from the DravoHastings Corporation at a list price of $\$ 13,157.50$. Currently discounts of up to $60 \%$ from the list prices are available. At list price the heater is about the same cost as the stack. Assuming the heater was operating about two months a year, the annual operating cost would be approximately $\$ 8,640$.

The financial analysis included three variations: a best, worst, and most likely case. The assumptions pertaining to each case are listed below: Best Case

- 4.5 feet diameter corrugated steel stack (30\% less than the plate steel stack cost)

- All equipment on site

- All labor on site

- Minimal transportation costs for materials

- No icing problem

- $2.5 \%$ increase in ventilation power costs

Worst Case

- 6.0 feet diameter carbon steel stack

- $20 \%$ increase in equipment cost for mobilization

- 20\% increase in labor cost for transportation to site

- 20\% increase in materials cost for transportation to site

- $80 \%$ increase in capital cost for heaters

- $10 \%$ increase in ventilation power costs

- 4 months per year operation of heaters $(\$ 17,280 / y r)$

Most Likely Case

- 6.0 feet diameter carbon steel stack

- $10 \%$ increase in equipment cost for mobilization

- $10 \%$ increase in labor cost for transportation to site

- 10\% increase in materials cost for transportation to site 
- $10 \%$ increase in capital cost for heaters

- $5 \%$ increase in ventilation power costs

- 2 months per year operations of heaters $(\$ 8640 / y r)$

The results of the financial analysis appear in Table 6.3 as a range of values. The costs are given in dollars per ton of ore as well as dollars per pound of $\mathrm{U}_{3} \mathrm{O}_{8}$.

TABLE 6.3. Increased Cost of Production Resulting From Adding Ventilation Stacks on Exhaust Boreholes

Best Case

$$
\begin{aligned}
& \$ 0.168-\$ 0.271 / \text { ton of ore } \\
& \$ 0.022-\$ 0.067 / \text { pound of } U_{3} 0_{8}
\end{aligned}
$$

Horst Case

$$
\begin{aligned}
& \$ 0.724-\$ 1.493 / \text { ton of ore } \\
& \$ 0.091-\$ 0.417 / \text { pound of } \mathrm{J}_{3} \mathrm{O}_{8}
\end{aligned}
$$

Most Likely Case

$\$ 0.493-\$ 0.881 /$ ton of ore

$\$ 0.062-\$ 0.242 /$ pound of $\mathrm{U}_{3} \mathrm{O}_{8}$

\section{$6.3222_{\text {RN EMISSIONS TO AIR FROM UNDERGROUND URANIUM MINES }}$}

\subsubsection{Methodology of Calculations}

The published information that has been located concerning radon emissions from specific underground uranium mines has been limited to two NUREG documents written by Jackson et al. $(1979,1980)$. In them are reported the resuits of measurements obtained by grab sampling air emitted from vents at uranium mines. These mines represented $65 \%$ of the total $\mathrm{J} . \mathrm{S}$. underground $1 \mathrm{I}_{3} \mathrm{O}_{8}$ production in 1979. The purpose of this chapter is to review the current status of those mines and to compute an estimate of the current radon emission rate from each of them. Table 6.4, taken from the original Table 6 of the 1980 document summarizes the status of the emissions at that time. The identity of the mines had been coded by agreement with the mine operators at the time permission was granted to make the original measurements. The results will be again reported in a coded fashion since mine operators have requested a continuation of their anonymity. 
TABLE 6.4. Summary of Radon Emissions from Underground Mine Vents

\begin{tabular}{|c|c|c|c|c|c|}
\hline Mine & $\begin{array}{c}1979 \\
\text { Measurement } \\
\text { Ci/yr } \\
\end{array}$ & $\begin{array}{c}1978 \\
\text { Measurement } \\
\text { Ci/yr } \\
\end{array}$ & $\begin{array}{r}\text { Overal } \\
\text { Average } \\
\text { Cilyr }\end{array}$ & & $\begin{array}{c}\text { Ratio } \\
1978-1979 \\
\end{array}$ \\
\hline A & $7,400 \pm 1100$ & & $7,400 \pm$ & 1100 & \\
\hline B & $4,700 \pm 60$ & $4,300 \pm 100$ & $4,500 \pm$ & 300 & $1.09 \pm 0.03$ \\
\hline C & $5,200 \pm 200$ & $3,900 \pm 300$ & $4,600 \pm$ & 800 & $1.33 \pm 0.11$ \\
\hline D & $3,630 \pm 120$ & & $3,630 \pm$ & 120 & \\
\hline $\mathrm{E}$ & $29,800 \pm 400$ & & $29,800 \pm$ & 400 & \\
\hline$F$ & $9,200 \pm 270$ & $9,500 \pm 200$ & $9,400 \pm$ & 200 & $0.97 \pm 0.03$ \\
\hline G & $2,150 \pm 50$ & $1,460 \star \star$ & $1,800 \pm$ & 400 & $1.47 \pm 0.03$ \\
\hline$H$ & $15,200 \pm 300$ & & $15,200 \pm$ & 300 & \\
\hline I & $1,690 \pm 80$ & & $1,690 \pm$ & 80 & \\
\hline J & $7,760 \pm 190$ & $8,100 \pm 400$ & $7,900 \pm$ & 200 & $0.96 \pm 0.05$ \\
\hline$k$ & $7,000 \pm 190$ & $5,870^{\star \star}$ & $6,400 \pm$ & 700 & $1.19 \pm 0.03$ \\
\hline L & $1,470 \pm 40$ & $1,320 \pm 30$ & $1,400 \pm$ & 300 & $1.11 \pm 0.05$ \\
\hline $\mathrm{M}-\mathrm{Q}$ & Not Sampled & & & & \\
\hline $\mathrm{R}$ & $15,000 \pm 400$ & 14,600 & $14,800 \pm$ & 300 & $1.03 \pm 0.04$ \\
\hline S & Not Sampled & & & & \\
\hline$T$ & $1,890 \pm 120$ & & $1,890 \pm$ & 120 & \\
\hline U & $890 \pm$ & & $890 \pm$ & 20 & \\
\hline V & $1,010 \pm$ & & $1,010 \pm$ & 60 & \\
\hline$W, X$ & Not Sampled & & & & \\
\hline$y^{\prime}$ & $17,500 \pm 400$ & & $17,500 \pm$ & 400 & \\
\hline Z & & $2,640 \pm 70$ & $2,640 \pm$ & 70 & \\
\hline$A A$ & $2,100 * *$ & $1,490 \pm 70$ & $1,800 \pm$ & 400 & 1.41 \\
\hline BB & $2,130 \pm$ & $1,840 \pm 70$ & $2,000 \pm$ & 200 & $1.16 \pm 0.06$ \\
\hline $\mathrm{CC}$ & & $2,120 \pm 50$ & $2,120 \pm$ & 50 & \\
\hline DD & & $960 \pm 40$ & $960 \pm$ & 40 & \\
\hline $\mathrm{EE}$ & $6,500 \pm$ & & $6,500 \pm$ & 70 & \\
\hline $\mathrm{FF}$ & $2,510 \pm$ & & $2,510 \pm$ & 80 & \\
\hline $\mathrm{GG}$ & $190 \pm$ & $146 \pm$ & $170 \pm$ & 30 & $1.30 \pm 0.05$ \\
\hline $\mathrm{HH}$ & $1,040 \pm$ & & $1,040 \pm$ & 60 & \\
\hline \multirow[t]{2}{*}{ I I } & $470 \pm$ & & $470 \pm$ & 10 & \\
\hline & & $\begin{array}{l}\text { SUM ALL MINES } \\
\pm \text { STD. DEV. }\end{array}$ & \multicolumn{2}{|c|}{$\begin{array}{r}15,000 \pm 2000 \\
( \pm 3000)\end{array}$} & $1.18 \pm 0.05$ AVE \\
\hline
\end{tabular}

* Single sample

** Average of sequential sample data, 1978

Source: Jackson, et al. (1980) 
To update the status, a representative of each mine operator was contacted by telephone (on or before August 16, 1983) and asked if their mine(s) were still operating. If a complete or partial shutdown was indicated, then the operator was asked if the mine vents and portals were operating or sealed. If a mine was shut down and an attempt made to seal all vents, ${ }^{22}{ }^{2} \mathrm{Rn}$ emissions to the surface were considered to be zero. If a mine was shutdown but with vents still operating either continuously or intermittently (i.e., not sealed), the mine was considered to be emitting radon.

For the shutdown but radon emitting mines, no attempt was made to quantify the reduction in radon emissions resulting from the decay of radionuclides in stagnated underground air. The reduction of radon due to decay is dependent on elapsed time of air stagnation which in turn depends on cycles of fan operation and the rate of natural draft ventilation.

For operating mines, the current emission rate will be considered as greater than the rate measured during the 1979 study because of a greater mine wall surface area exposed by continuing mining. The rate of increase will be estimated for those mines that are operating in 1983 and that provided annual production rate data in the 1979 study (see Table 1 of Jackson, et a1. 1980). In addition, new data have been received from these current operating mines.

Some mine operators indicated that their current mine emissions should be reduced from those measured in 1978 and 1979 because the current mine production rate is smaller and/or that areas of their mine are inactive or not being ventilated. However, except where specific details about the bulkheading and other measures taken to prevent the passage of radon to the surface were available, it was not possible to predict whether radon from the unused areas of a mine is decaying underground or if it is reaching the surface. Since that information has not been received for these mines in question at the time of this report, radon emissions from these mines will be calculated using the same assumptions as for operating mines.

Table 6.5 shows the current status of the mines from the 1978-1979 study. Based on the Mine Survey data in Table 1 of the 1980 report, the total daily ore production in 1979 of the mines which are still operating today was 8500 tons. This is equivalent to $2,125,000$ tons ore per year (based on 250 
TABLE 6.5. Current (1983) Status of Mines Monitored in

1978-1979 Survey of Jackson, et al. (1979, 1980)

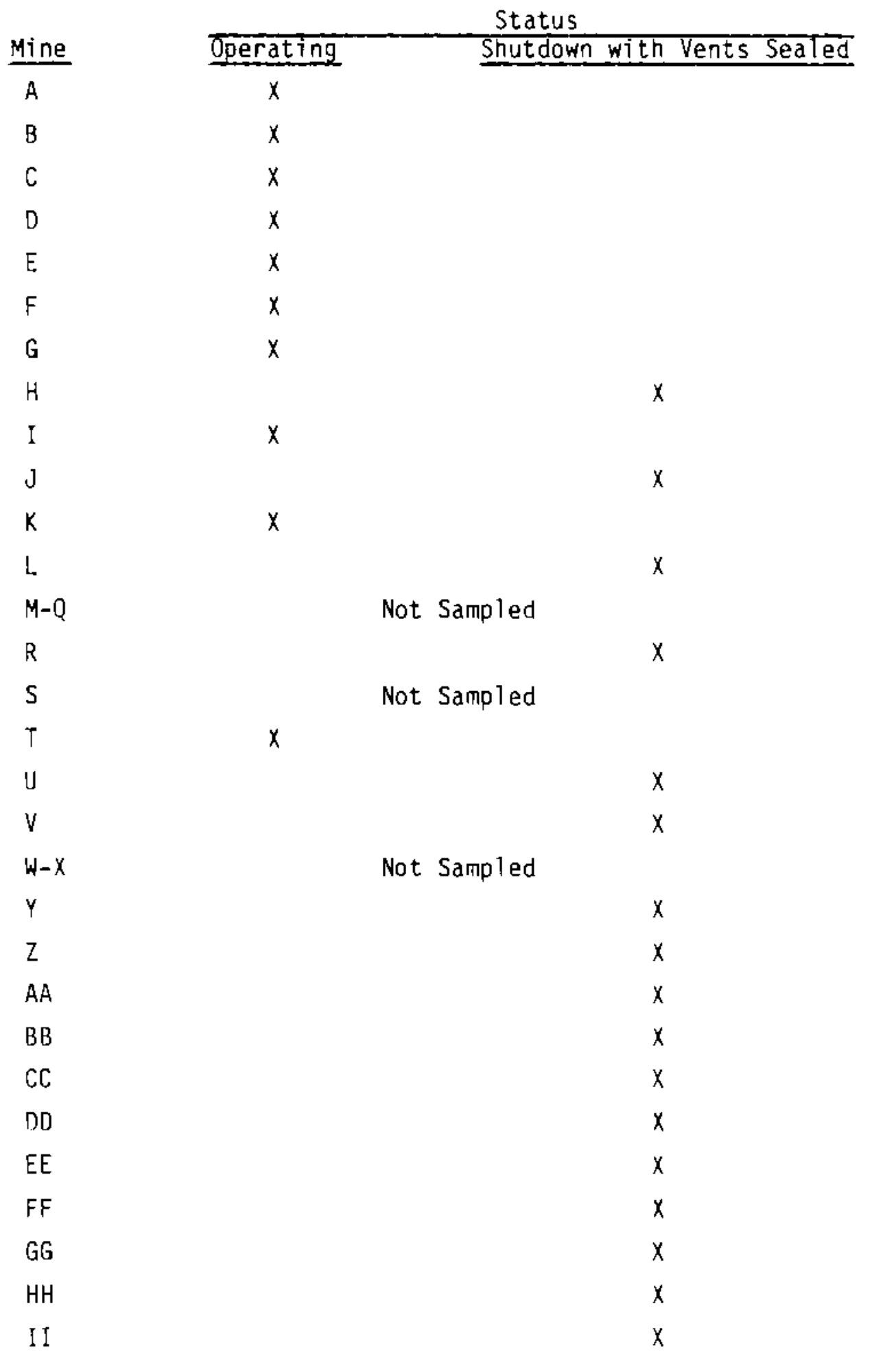


operating days per year) and is $59 \%$ of the total production of $3,600,000$ tons ore per year of all mines where vents were monitored in 1979 and 1980.

It is difficult to forecast the future status of these mines, given the economic circumstances of the uranium industry. For the purposes of predicting future emissions, it will be assumed that the mines in the survey, operating in 1983, will remain open through 1990, and that economics will dictate production rate changes in proportion to the changes forecast for the entire industry by the Department of Energy (1983).

The prediction of current and future ${ }^{222} \mathrm{Rn}$ radon emission rates from measurements taken 4 to 5 years ago, requires simplifying assumptions that are not necessarily accurate for individual mines. Those assumptions have been discussed in the section of this report covering techniques for monitoring radon emissions. Basically, it is assumed that each mine will increase in size by an increment of annual ore production and that the radon emission rate will also increase. Using the relationship between the cumulative production of ore and the radon emission rate $\left(0.44 \times 10^{-2} \mathrm{Ci} /\right.$ year ton of ore) determined by Jackson et al. (1980), one can predict the annual increase of radon emissions from the annual production listed for each mine in Table 1 taken from the same document. This emission rate coefficient was computed for the total emissions from both above-ground sources and ventilation areas. However, since ventilation air was estimated to be $98 \%$ of the total emanation rate reported for 1978 and 1979, the error caused by above-ground sources is negligible.

It is unlikely that the annual production rate from each mine remained fixed during its life in the years following 1978. Since new production statistics for each mine have not currently been received, the 1978 production rates are adjusted in the current calculation to reflect the ratio of the national total production statistics for all underground mines in a given year to that for 1978 as given by the Department of Energy (DOE 1983). These data are summarized in Table 6.6. Also shown in this table, in column four, are the products of the predicted fractions of 1978 production for each year multiplied by the emission rate coefficient for 1978-1979. Multiplying the 1978 annual production rate for a mine by the factors in column four will yield an estimate for the annual increments of radon emission rate to be added to the measured 
TABLE 6.6. Uranium Ore Produced from Underground Mines and

Fractional Radon Emission Rate Factors

\begin{tabular}{|c|c|c|c|c|}
\hline & Year & $\begin{array}{l}\text { Ore Production } \\
\text { (thousands tons) }\end{array}$ & $\begin{array}{c}\text { Fraction of } \\
1978 \text { Production } \\
\end{array}$ & $\begin{array}{c}\text { Emission Rate Factor } \\
\text { (Fraction of } 1978 \\
\times \text { Ci/yr ton) }\end{array}$ \\
\hline \multicolumn{2}{|r|}{$\begin{array}{l}1978 \\
1979 \\
1980 \\
1981 \\
1982 \\
1983(\mathrm{a})\end{array}$} & $\begin{array}{l}6,105 \\
5,356 \\
6,351 \\
5,229 \\
2,809\end{array}$ & $\begin{array}{l}1.00 \\
0.83 \\
1.04 \\
0.86 \\
0.46\end{array}$ & $\begin{array}{l}0.44 \times 10^{-2} \\
0.39 \times 10^{-2} \\
0.46 \times 10^{-2} \\
0.38 \times 10^{-2} \\
0.20 \times 10^{-2}\end{array}$ \\
\hline & $\begin{array}{l}1983(a) \\
1984(a) \\
1985(a) \\
1986(a) \\
1987(a) \\
1988(a) \\
1989(a) \\
1990(a)\end{array}$ & $\begin{array}{l}2,208 \\
2,011 \\
1,719 \\
1,695 \\
1,672 \\
1,901 \\
1,874 \\
1,846\end{array}$ & $\begin{array}{l}0.36 \\
0.33 \\
0.28 \\
0.28 \\
0.27 \\
0.31 \\
0.31 \\
0.30\end{array}$ & $\begin{array}{l}0.16 \times 10^{-2} \\
0.15 \times 10^{-2} \\
0.12 \times 10^{-2} \\
0.12 \times 10^{-2} \\
0.12 \times 10^{-2} \\
0.14 \times 10^{-2} \\
0.14 \times 10^{-2} \\
0.13 \times 10^{-2}\end{array}$ \\
\hline$\Sigma \quad 1979$ & through 1983 & 21,953 & 3.60 & $1.58 \times 10^{-2}$ \\
\hline$\sum 1979$ & through 1990 & 34,671 & 5.68 & $2.50 \times 10^{-2}$ \\
\hline
\end{tabular}

(a) Uranium ore produced from underground mines is based on an estimated total industry $\mathrm{U}_{3} 0_{8}$ production for 1983 of 10,600 tons and for 1990 of 9600 tons. For intermediate years annual $\mathrm{U}_{3} \mathrm{O}_{8}$ production rates were interpolated between the 1983 and 1990 values. The conversion from total industry tons $\mathrm{U}_{3} \mathrm{O}_{8}$ to total industry tons ore was made using average ore grades predicted for each year obtained via personal communication from the DOE Grand Junction office. Finally, the percentage of total industry ore attributed to underground mining operations was estimated at $25 \%$ for the years between 1983-1990. This percentage was estimated from the trend in historical data from the DOE (1983) on underground versus open-pit mining.

radon emission rate in 1978 for that mine. The sum of these factors for years 1979 through 1983 or 1990 yields the expected added increment of ${ }^{222}$ Rn emission during the intervals between the original measurement set of Jackson et al. (1980) and December 31, 1983 or December 31, 1990, respectively.

\subsubsection{Calculation Process}

The calculation process for predicting 1983 and $1990222_{R n}$ emission rates for each mine in the 1978-79 Jackson et al (1980) study will be outilined. 
First, the mine's given 1978 daily ore rate is converted to an annual rate:

$$
(D P R)_{j}(O P D)=(A P R)_{i}
$$

where

$$
\begin{aligned}
& \text { DPR }=1978 \text { daily production rate per mine } i \text { in tons ore/day } \\
& O P D=250 \text { operating days per year } \\
& A P R=1978 \text { annual production rate per mine } i \text { in tons ore/day }
\end{aligned}
$$

Then by using the calculated cumulative fractional radon emission rate factor for 1979-1983 and 1979-1990 (see Table 6.6), a cumulative increase of annual rates of ${ }^{22}{ }^{2} \mathrm{Rn}$ emissions per mine since 1978 can be obtained:

$$
(A P R)_{i}(C F R E)_{x}=(C A R E)_{i}
$$

where

$$
\begin{aligned}
\text { CFRE = } & \text { cumulative fractional radon enission rate factor for period } x \\
& (1979-1983 \text { and } 1979-1990) \\
\text { CARE = } & \text { cumulative increase of annual }{ }^{222} \text { Rn emission rates per mine } i \\
& \text { since } 1978, \text { in } \mathrm{Ci} / \mathrm{yr} .
\end{aligned}
$$

Finally, the (CARE) $i$ figure is added to the mine's 1978 reported ${ }^{222}$ Rn emission rate:

$$
(\text { CARE })_{i}+\langle\text { RER })_{i}=(\text { TRER })_{i}
$$

where

$$
\begin{aligned}
& \text { RER }=1978{ }^{222_{R n}} \text { emission rate per mine } i \text { in } \mathrm{Ci} / \mathrm{yr} \text {. } \\
& \text { TRER = new total }{ }^{222} \text { Rn enission rate per mine } i \text { as of December } 31,1983 \\
& \text { and December 31, } 1990 \text { in } \mathrm{Ci} / \mathrm{yr} \text {. }
\end{aligned}
$$

The result (TRER) $i$ is the predicted total ${ }^{22} 2_{R n}$ emission rate for each mine at the end of years 1983 and 1990. 


\subsubsection{Results and Discussion}

The results of this computation process for all the mines sampled in 1978 and 1979 are shown in Table 6.7. These computed extrapolations predict that the total emission rate for the mines sampled will be $70 \%$ of the 1978 and 1979 rate on December 31, 1983 and $83 \%$ of the 1978 and 1979 rate on December 3l, 1990. This assumes that all the mines for which computations were made continue to operate, and that their production follows the industry averages. It can be argued that the production cutbacks observed in the national totals occur as a result of mine closures. However, in discussions with representatives of operating mine companies, a frequently heard comment was that production from the mines was being significantly reduced at the present time. Moreover, several new mines have started producing since the 1978 and 1979 surveys. Whether these new mines represent a significant fraction of the production lost from closed mines is not known at the present time. The radon produced by a new mine would initially be expected to be lower than a similarly sized operation that was closing after several years of productive life. It can be seen in Table 6.7 that the predicted emission rate increases much more rapidly for the four-year-old mine $G$ than for a 20 -year-old mine such as $F$.

\subsubsection{Conclusions}

The model used for predicting future emissions from past measurements uses only the trend observed in the data of the 1978 and 1979 study, which predicts an ever increasing release of ${ }^{222}$ Rn from a mine unless areas are completely sealed from the surface. Since actual current production statistics were not available for each mine, and the suitability of the model to individual operations has not been tested, these predictions must be viewed as tentative and suitable only for industry-wide predictions. Based on the predictions, radon emission rates for individual operating mines are increasing but a significant fraction of mines is no longer operating and most of these mines are sealed. Additional onsite inspection of the degree of sealing for "closed mines" should be performed to verify that bulkheads and seals are adequate. Current measurements at operating mines, along with production statistics up to the present time, should be collected to demonstrate the suitability of the model to industry-wide projections. However, only a detailed research program could improve 
TABLE 6.7. Measured and Predicted ${ }^{222}$ Rn Emission Rates

\begin{tabular}{|c|c|c|c|c|}
\hline Mine & $\begin{array}{c}\text { Production } \\
\text { Rate (hundred } \\
\text { thousand tons/yr) }\end{array}$ & $\begin{array}{c}\text { Emission Rate } \\
\text { on } 12-31-78 \\
\text { (thousands } \mathrm{Ci} / \mathrm{yr} \text { ) } \\
\end{array}$ & $\begin{array}{l}\text { Predicted Emission } \\
\text { Rate on } 12-31-83 \\
\text { (thousands } \mathrm{Ci} / \mathrm{yr} \text { ) }\end{array}$ & $\begin{array}{l}\text { Predicted Emission } \\
\text { Rate on } 12-31-90 \\
\text { (thousands } \mathrm{Ci} / \mathrm{yr} \text { ) }\end{array}$ \\
\hline A & 5.5 & 7.4 & 16.0 & 21.1 \\
\hline 8 & 1.78 & 4.5 & 7.3 & 9.0 \\
\hline C & 2.36 & 4.6 & 8.3 & 10.5 \\
\hline $\mathrm{D}$ & 2.68 & 3.63 & 7.9 & 10.3 \\
\hline$E$ & 2.50 & 29.8 & 33.8 & 36.1 \\
\hline$F$ & 1.79 & 9.4 & 12.2 & 13.9 \\
\hline$G$ & 1.98 & 1.80 & 4.9 & 6.8 \\
\hline$H$ & 1.20 & 15.2 & 0 & 0 \\
\hline I & 0.75 & 1.69 & 2.88 & 3.57 \\
\hline J & 0.92 & 7.9 & 0 & 0 \\
\hline$k$ & 0.88 & 6.4 & 7.8 & 8.6 \\
\hline L & 0.62 & 1.4 & 0 & 0 \\
\hline$M-Q$ & & Not Sampled & & \\
\hline$R$ & 0.28 & 14.8 & 0 & 0 \\
\hline $\mathrm{S}$ & & Not Sampled & & \\
\hline $\mathrm{T}$ & 1.05 & 1.89 & 3.55 & 4.2 \\
\hline U & 1.25 & 0.89 & 0 & 0 \\
\hline V & 1.38 & 1.01 & 0 & 0 \\
\hline$W-X$ & & Not Sampled & & \\
\hline Y & 6.6 & 17.5 & 0 & 0 \\
\hline 2 & 1.25 & 2.64 & 0 & 0 \\
\hline AA & -- & 1.80 & 0 & 0 \\
\hline $\mathrm{BB}$ & $-\infty$ & 2.00 & 0 & 0 \\
\hline $\mathrm{CC}$ & -- & 2.12 & 0 & 0 \\
\hline 00 & -- & 0.96 & 0 & 0 \\
\hline $\mathrm{EE}$ & -- & 6.50 & 0 & 0 \\
\hline $\mathrm{FF}$ & -- & 2.51 & 0 & 0 \\
\hline $\mathrm{GG}$ & -- & 0.17 & 0 & 0 \\
\hline$H H$ & -- & 1.04 & 0 & 0 \\
\hline I I & -- & 0.47 & 0 & 0 \\
\hline Total & & 150 & 105. & 124. \\
\hline
\end{tabular}


the model by adding the significant parameters in addition to the quantity of ore extracted. Again, we caution that the predicted December, 1983 emission rates are subject to large uncertainties and that actual field measurements are required to obtain accurate emission rates. 


\subsection{REFERENCES}

American Mining Congress Journa1. 1983. "AMC Uranium Counci1 to Gather Data." American Mining Congress Journal. March 2, 1983.

Anderson, J.R. and J. Q. Jones. 1980. "Uranium Production" in Uranium Industry Seminar, Report No. GJO-108(80), U.S. Department of Energy, Grand Junction, Colorado.

Atlas Corporation. 1982. Annual Report. At las Corporation, Princeton, New Jersey.

Aucoin, P. A. 1982. "Uranium--Just Another Metal?" Paper presented at the Uranium Colloquium V, October 6 and 7, 1982, Grand Junction, Colorado, sponsored by Nuclear Assurance Corporation, Atlanta, Georgia.

Battelle Columbus Laboratories. 1976. Assessments of Environmental Aspects of Uranium Mining and Milling. PB-266 413, Prepared for Industrial Environmental Research Laboratories, Resource Extraction and Handling Division, Cincinnat $i$, Ohio.

Bedore, J. 1982. "Domenici and Protectionism." Paper Presented at the Uranium Colloquium V. October 6 and 7, 1982, Grand Junction, Colorado, sponsored by Nuclear Assurance Corporation, Atlanta, Georgia.

Bengston, S. A. 1979. "Irrigation Techniques for Tailings Revegetation in the Arid Southwest." Tailings Disposal Today. Vol. 2, pp. 487-504, Miller Freeman Publications, Incorporated, San Francisco, California.

Bock, T. 1978. "Perspectives of the Uranium Industry." In Proceedings: First Uranium Resource/Technology Seminar. March, 1978, GoTden, Colorado, Colorado School of Mines Press, GoTden, Colorado.

Bonny, J., and M. Fulton. 1982. "The Uranium Equation in 1982." Paper presented at the Uranium Institute's Seventh Annual Symposium, September 1-3, 1982, London, England. Sponsored by Uranium Institute, London, England.

Breslin, J. A. 1981. "Bureau of Mines Statement of Principles," In Radiation Hazards in Mining: Control, Measurement, and Medical Effects, ed, Manual Comez, pp. 15-16, Society of Mining Engineers, Anerican Institute of Mining, Metallurgical and Petroleum Engineers, Incorporated New York.

Brown, R. W., and R. H. Williamson. 1977. "Domestic Uranium Requirements." In Uranium Industry Seminar, Report No. GJ0-108(77), U.S. Department of Energy, Grand Junction, Colorado.

Bruno, R. C., M. Israeli and P. J. Magno. 1983. "Improval Procedures for Estimating Annual Average Concentrations of Radon and Its Decay Products in Houses." Presented at Health Physics Society Annual Meeting, Baltimore, Maryland, 19-23 June 1983. Health Physics Society, Bethesda, Maryland. 
Busigin, A., A. W. Van der Vooren and C. R. Phillips. 1979. "Interpretation of the Response of Continuous Radon Monitors to Transient Radon Conditions." Health Physics. Vol. 37, No. 5.

Bykhovskii, A. U., N. T. Chesnokov, et al. 1974. Experiences with Radon Control in Mining. Published for the U.S. Bureau of Mines and the National Science Foundation, Washington D.C. by Amerind Publishing Co. PVT. LTD, New Delhi, India.

Catalano L. 1983a. "Fuel Forecasters Warn of Obstacles to Future Supply." Power, January, 1983, pp. 49-52.

Catalano, L. 1983b. "Nuclear Shows Little Improvement Under Reagan." Power, January, 1983, pp. 13-14.

Charles River Associates, Incorporated 1977. Uranium Price Formation. Report No. EPRI EA-498, Electric Power Research Institute, Palo Alto, California.

Chemical Engineering. 1981. "Economic Indicators," Chemical Engineering, November 2, 1981, p. 7.

Chemical Engineering. 1982. "Economic Indicators," Chemical Engineering, November 15, $1982, \mathrm{p} .7$.

Clark, G. C., and A. W. Reynolds. 1978. "Uranium Market--Domestic and Foreign Requirements." In Uranium Industry Seminar, Report No. GJ0-108(78), U.S. Department of Energy, Grand Junction, Colorado.

Clark G. C., and A. W. Reynolds. 1979. "Uranium Market Forecast." In Uranium Industry Seminar, Report No. GJ0-108(79), U.S. Department of Energy, Grand Junction, Colorado.

Cheney, E. S., and L. S. Istas. 1980. "Temporal and Lithologic Envi ronments of Uranium Ore Oeposits." In Analys is of AEC-ERDA-OOE and USGS Uranium Program Data. Report No. EPRI FA-1374, pp. 2-1 to 2-77, Electric Power Research institute, Palo Alto, California.

Connor, M. J. 1983. "World Uranium Markets: An Update." In Proceedings: Fuel Supply Seminar. October, 1982, St. Louis, Missouri, Report No. EPRT-EA 2994, Electric Power Research Institute, Palo Alto, California.

Consumers Power Company. 1982. Securities and Exchange Commission Form 10-k Annual Report, Consumers Power Company, Jackson, Michigan.

Dames and Moore. 1980. Analysis of AEC-ERDA-OOE and USGS Uranium Program Data. Report No. EPRI EA-1374, Electric Power Research Institute, Palo Alto, California.

DeVergie, P. C., et al. 1982. "Production Capability of the U.S. Uranium Industry." Paper presented at Uranium Colloquium V, October 6-7, 1982, Grand Junction, Colorado, sponsored by Nuclear Assurance Corporation, Atlanta, Georgia. 
Doiron, D. G. 1978. "Utility Involvement in Uranium Production: An Overview-Progress and Potential." Paper presented at American Nuclear Society Topical Meeting, September 10-13, 1978, Las Vegas, Nevada. Anerican Nuclear Society, La Grange Park, Illinois.

Dykstra, F. R. and R. F. Tatna11, "Ferroalloy Ores." In Economics of the Mineral Industries, 3rd edition, ed. William A. Vogely, pp. 519-531, American Institute of Mining, Metallurgical, and Petroleum Engineers, Incorporated New York.

E \& MJ. 1981. International Directory of Mining. Engineering and Mining Journal, Mining Information Services, New York, New York.

E \& MJ, 1983. "Lack of Ore Forces Temporary Closing of Blanding Uranium Mi11," E \& MJ, February 1983, p. 37.

Electric Power Research Institute (EPRI). 1982. Technical Assessment Guide. Report No. EPRI P-2410-SR. Electric Power Research Institute, Palo Alto, Cal ifornia.

Energy and Minerals Department. 1982. Annual Resources Report. Energy and Minerals Department, Santa Fe, New Mexico.

Engelsman, C. 1981. 1981 Heavy Construction Cost File. Van Nostrand Reinhold Co., New York, NY.

Jet Propulsion Laboratory (JPL). 1976. The Cost of Energy from Utility-0wned Solar Electric systems, A Required Revenue Methodology for ERDA/EPRI Evaluations. Report No. JPL 5040-29, Prepared by the Jet Propulsion Laboratory, California Institute of Technology, Pasadena, California, for the U.S. Energy Research and Development Administration, Division of Solar Energy, Washington D.C.

Facer, J. F. Jr. 1978. "Trends in Uranium Production," in Uranium Industry Seminar, Report No. GJ0-108(78), U.S. Department of Energy, Grand Junction, Colorado.

Facer, J. F. Jr. 1979. "Uranium Production," in Uranium Industry Seminar, Report No. GJ0-108(79), U.S. Department of Energy, Grand Junction, CoTorado.

Federal Register. 1983. "Part II, Environmental Protection Agency, National Emission Standards for Hazardous Air Pollutants; Standards for Radionuclides." In Federal Register, Vol. 48, No. 67, Wednesday, April 6, 1983, pp. 15076-15091, Office of the Federal Register, National Archives and Records Service, General Services Administration, Washington, 0.C.

Fisenne, I. M., A. C. George and H. W. Keller. 1983. Radon Intercomparison Exercise at EML, Report No. EML-413, Environmental Measurements Laboratory, U.S. Department of Energy, New York, N.Y. 
Floyd C. Bossard \& Associates, Incorporated. 1983. A Manual of Mine Ventilation Design Practices. 2nd ed., Floyd C. Bossard and Associates, Incorporated, Butte, Montana.

Gilman, P., Dr. 1982. "An Address." Paper presented at the Uranium Colloquium V, October 6 and 7, 1982. Grand Junction, Colorado, sponsored by Nuclear Assurance Corporation, Atlanta, Georgia.

Goodier, John T. 1978. Wyomings' Uranium Industry--Status, Impacts, and Trends, State of Wyoming Department of Economic Planning and Development, Minerals Division, Laramie, Wyoming.

Gordon, Emanua1. 1977. "Uranium-Rising Prices Continue to Support Development Activity". E\&MJ, March, 1977, pp.194-200.

Gulf 0il Corporation. 1982. Securities and Exchange Commission Form 10-K Annual Report, Gulf $0 i 1$ Corporation, Pittsburgh, Pennsylvania.

Hahne, F. J. III. 1982. "Future Uranium Prices." Paper presented at Uranium Colloquium V, October 6-7, 1982, Grand Junction, Colorado, sponsored by Nuclear Assurance Corporation, At lanta, Georgia

Hanrahan, E. J. 1975. "Domestic and Foreign Uranium Requirements, 19752000." In Uranium Industry Seminar, Report No. GJ0-108(75), U.S. Department of Energy, Grand Junction, Colorado.

Hanrahan, E. J., R. H. Williamson and R. W. Brown. 1976. "United States Uranium Requirements." In Uranium Industry Seminar, Report No. Gjo-108(76), U.S. Department of Energy, Grand Junction, Colorado.

Hanrahan, E. 3. 1982. "Uranium in the Nation's Energy Future," Paper presented at Uranium Colloquium V, October 6-7, 1982, Grand Junction, Colorado, sponsored by Nuclear Assurance Corporation, Atlanta, Georgia.

Harris, R. L., and R. E. Bales. 1964. "Uranium-Mine Ventilation for Control of Radon and Its Daughter Products." In Radiological Health and Safety in Mining and Milling of Nuclear Materials, $\overline{p P} \cdot 49-60$. International Atomic Energy Agency, Vienna, Austria.

Heeb, C. M., R. L. Aaberg, et aT. 1980. A Survey of LWR Environmental Control Technology Performance and Cost. Report Nö. PNL-2287, Pacjfic Northwest Laboratories, Richland, Washington.

Heiks, R. L. 1982. Paper presented at Uranium Colloquim V. October 6-7, 1982, Grand Junction, Colorado, sponsored by Nuclear Assurance Corporation, At lanta Georgia.

Homestake Mining Company. 1982. Securities and Exchange Commission Form 10-K Annual Report. Homestake Mining Company, San Francisco, California. 
Hulse, R. D. 1982. "Uranium Supply-The Roller Coaster Effect." Paper presented at the Uranium Colloquium V. October 6 and 7, 1982. Grand Junction, Colorado, Colorado, sponsored by Nuclear Assurance Corporation, At lanta Georgia.

Ibbotson, R. F., and R. A. Sinquefield. 1977. Stocks, Bonds, Bills and Inflation: The Past $(1926-1976)$ and the Future $(1977-2000)$. Financial Analysts Research Foundation, Charlottesville, Virginia.

Jackson, P. 0., et al. 1979. Radon-222 Emission in Ventilation Air Exhausted from Underground Uranium Mines. Report No . NOREG/CR-0627, PNL-2888 Rev., Pacific Northwest Laboratory, Richland, Washington.

Jackson, P. 0. et al. 1980. An Investigation of Radon-222 Emissions from Underground Uranium Mines. Report No. NUREG/CR-1273, PNL-3262, Pacific Northwest Laboratory, Richland, Washington.

Kay, Burgess L. 1981. "Mulch and Chemical Stabilizers for Land Reclamation in Dry Region." Reclamation of Drastically Disturbed Lands. Pp. 467-483. Eds. R. G. Dinaver et a7. American Society of Agronomy, Crop Society of America, Incorporated, Soil Society of America, Incorporated, Madison, Wisconsin.

Kovisars, Leons. 1979. "Uranium for Nuclear Power, 1979 - 2000", In Proceedings: Second Uranium Resource/Technology Seminar, March, 1979, Golden, Colorado, Colorado School of Mines Press, Golden, Colorado.

Kerr-McGee Corporation. 1982. Securities and Exchange Commission Form 10-K Annual Report. Kerr-McGee Corporation, OkTahoma City, OkTahoma.

Lang E. A. 1982. "The Case for Import Restriction." Paper presented at Uranium Colloquium V, Detober 6-7, 1982, Grand Junction, Colorado, Colorado, sponsored by Nuclear Assurance Corporation, Atlanta Georgia.

Leamon G. E., and R. M. Casper. 1982 "Uranium Demand Projections Through 2000." Paper presented at Uranium Colloquium V, October 6-7, 1982, Grand Junction, Colorado, Colorado, sponsored by Nuclear Assurance Corporation, At lanta Georgia.

McGinley, F.E. 1975. "Effects of Stricter Radiation Exposure Standards on Uranium Costs and Ore Reserves," In Radon In Uranium Mining Proceedings of a Pane?, pp. 3-14, International Atomic Energy Agency, Vienna, Austria.

Martin, L. H. 1982. "The Embargo Issue from EEI's Perspective." Paper presented at the Uranium Colloqujum V. October 6 and 7, 1982. Grand Junction, Colorado, Colorado, sponsored by Nuclear Assurance Corporation, Atlanta Georgia. 
Mays, D. A., and G. W. Bengston. 1978. "Lime Fertilizers for Use in Land Reclamation in Humid Regions." In Reclamation of Drastically Disturbed Lands. pp. 307-327. Eds. R. G. Dinauer et a1. American Society of Agronomy, Crop Society of America, Incorporated, Soil Society of America, Incorporated, Madison, Wisconsin.

Means, R. S. 1982. Means Site Work Cost Data 1982. Robert Snow Means Company, Incorporated Kinston, Massachusetts.

Merritt, R. C. 1971. The Extractive Metallurgy of Uranium, Colorado School of Mines Research Institute, Golden, Colorado.

Millar, Rodney D., et. al. 1980, A Study of the Utah Uranium Milling Industry, Volume II, Utah Energy Resources - Uranium, prepared and published by Mechanical and Industrial Engineering Department and utah Engineering Experiment Station, University of Utah, Salt Lake City, Utah.

Mommsen, R. W., and J. T. Mommsen. 1980. "American Electric Utilities in Uranium Supply Ventures." Public Utilities Fortnightly. April 10, 1980, pp. 17-21.

National Crushed Stone Association. 1978. Quarried Stone for Erosion and Sediment Control. National Crushed Stone Association, Washington, 0.C.

Navajo and Hopi Indian Relocation Commission. 1981. 6th Annual Report, commissioners Roger K. Lewis, Hawley Atkjnson and Sandra L. Massetto, Navajo and Hopi Relocation Commission, Flagstaff, Arizona.

New Mexico Energy and Mineral Dept. 1979. An Overview of the New Mexico Uranium Industry. University of New Mexico Printing Plant, Santa Fe, New Mexico.

Newsweek. 1983. "A Blow to Nuclear Power." Newsweek, May 2, 1983, pp. 67-68.

Nuclear Fuel. 198la. "Bank's Role in Uranium Mining Is Expanding, Says a Banker who Thinks \$25/1b. Is Plenty." Nuclear Fuel. October 26, 1981.

Nuclear Fuel . 1981b. "Competitiveness, Not Contracts, Attract Financiers." Nuclear Fuel. September 28, 1981.

Nuclear Fue1. 1981c. "Industry Splits and Diplomats Join the Fray as hearings Open on Uranium Import Limits." Nuclear Fuel. October 21, 1981.

Nuclear Fuel. 1982a. "Embargo 1982, Uncertainty is the Intent." Nuclear Fuel. September 27, 1982.

Nuclear Fuel. 1982b. "House Rejects Uranium Import Ban." Nuclear Fuel. December 6, 1982.

Nuclear Fuel. 1983a. "Import Limiters Turn Attention to D0E." Nuclear Fuel. January 17, 1983. 
Nuclear FueT. 1983b. "Report Says Prolonged Inventory Selling Means Steeper Price Rise When It's Over." Nuclear Fuel, January 3, 1983, pp. 7-9. Originally cited in Natural Uranium, Demand, Supply and Price, 1982, Pickard Lowe \& Garrick Incorporated, Washington, D.C. and Colorado Nuclear Corporation, Monument, Colorado.

Nuclear News. 1982. "Hard Talk about a Softening Market." Nuclear News. September 13, 1982. pp. 4-8.

Nuclear News. 1983a. "EPA Establi ishes Radionuclide Limits," Nuclear News, May, 1983, pp. 94-95.

Nuclear News. 1983b. "NERA Says Nuclear Edge Over Coal May End." Nuclear News, March, 1983, p. 50.

Nuclear News. 1983c. "U Prices Continue Upswing." Nuclear News, March, 1983, p. 94.

Nuclear News. 1983d. "World List of Nuclear Power Plants." Nuclear News. February, 1983, pp. 71-90.

Packer, P. E., and E. F. Aldon, 1979. "Revegetation Techniques for Dry Regions." In Reclamation of Drastically Disturbed Lands. Pp. 425-449, Eds. R. G. Oinauer et al., American Society of Agronomy, Crop Society of America, Incorporated, Soil Society of America, Incorporated, Madison, Wisconsin.

Page, J. S. 1977. Estimator's General Construction Man-Hour Manual. Second Edition, Gulf Publishing Co., Houston, IX.

Perkins, B. L. 1979. An Overview of the New Mexico Uranium Industry. New Mexico Energy and Minerals Department, Santa Fe, New Mexico.

Persse, F. H., 0. W. Lockard and A. E. Lindquist. 1977. Coal Surface Mining and Reclamation Costs in the Western United States. Report No. IC 8737 , U.S. Department of the Interior, Bureau of Mines, washington, D.C.

Phelps Dodge Corporation, 1982. Securities and Exchange Commission Form 10-K Annual Report, Phelps Dodge Corporation, New York.

Phillips Petroleum Company. 1982. Securities and Exchange Commission Form I0-K Annual Report, Phillips Petroleum Company, Bartlesville, Oklahoma.

Press, A., et al. 1983. "A Blow to Nuclear Power." Newsweek, May 2, 1983, p. 67-68.

Raloff, J. 1983. "Nuclear Power at 25." Science News, January 1, 1983, pp. 12-13.

Rio Algom, Ltd. 1982. Securities and Exchange Commission Form 10-K Annual Report, Rio Algom, Ltd., Toronto, Ontario, Canada. 
Robertson, J. L. 1979. "Three in One Plant Produces Crushed Stone, Riprap, and Lime." Rock Products, $\vee 82(11)$, pp. 64-68.

San Juan Basin Regional Uranium Study--0ffice of Trust Responsibilities. 1980. Uranium Development in the San Juan Basin Region, A Report on Environmental Issues. U.S. Department of the Interior, Bureau of Indian Affairs, Lead Agency, Albuquerque, New Mexico.

Scarborough, R. B. 1981. Radioactive Occurrences and Uranium Production in Arizona, Report No. GJBX-143(81), Arizona Bureau of Geology and Mineral Technology, Tucson, Arizona.

Schneider, K. J., and T. J. Kabele. 1979. Description of Reference LWR Facilities for Analysis of Nuclear Fuel Cycles. Report No. PNL-2286, Pacific Northwest Laboratory, Rich]and, Washington.

Silver King Mines, Incorporated. 1982. Annual Report, Silver King Mines, Incorporated, Salt Lake City, Utah.

Staggs, J. J. 1980. "Uranium Demand." In Uranium Industry Seminar, Report No. GJ0-108(80), U.S. Department of Energy, Grand Junction, Colorado.

Staggs, J. J. 1981. "Uranium Demand Projections." In Uranium Industry Seminar, GJo-108(81), U.S. Department of Energy, Grand Junction, Colorado.

Stermole, Franklin J. 1983. "Mineral Investment 1983 Depends on Prices." Mining Engineering. February, 1983.

Steyn, J. J., et al. 1981. Intermediate-Term Uranium Supply Curve Estimation, Report No. EPRI EA-1706, Electric Power Research Institute, Palo Alto, California.

Stoller, S. M., and J. F. Hogerton. 1977. "Uranium Availability for Power Generation." Public Utilities Fortnightly. April 14, 1977, pp. 34-37.

Stout, K. S. 1980. Mining Methods and Equipment. McGraw-Hill, Incorporated, New York, New York.

Thomas, J. W. 1977. A System for Continuous Radon Determinations. Report No. HASL-327. Health and Safety office, J.S. Energy research and Deve lopment Administration, New York, New York.

Thrush, P. W., ed. 1968. A Dictionary of Mining, Mineral and Related Terms, U.S. Department of the Interior, Bureau of Mines, Washington, D.C.

UNC, Resources, Incorporated. 1982. Securities and Exchange Commission Form 10-K Annual Report. UNC Resources, Incorporated, Falls Church, Virginia.

Uranium Institute, Supply and Demand Committee. 1979. The 8alance of Supply and Demand, 1978 - 1990. Mining Journal Books, Ltd, Edenbridge, Kent,

England. 
Uranium Institute, The Supply and Demand Committee. 1981. The Uranium Equation, 1980-1995, Mining Journal Books, Ltd. Edenbridge, Kent, England.

U.S. Atomic Energy Commission (AEC). 1974. Environmental Survey of the Uranium Fuel Cycle. Report No. WASH-1248, U.S. Atomic Energy Commission, Washington, D.C.

U.S. Congress. Subcomnittee on Energy of the Joint Economic Comittee. 1975. Hearings. November 19, 1975.

U.S. Department of Commerce. 1978a. Business Conditions Digest. (August 1978). U.S. Department of Commerce, Washington, D.C.

U.S. Department of Commerce, Bureau of the Census. 1978b. Taxable Property Values and Assessment/Sales Price Ratios, 1977 Census of Governments, Report No. GC77(2), U.S. Department of Commerce, Bureau of Census, Washington, D.C.

U.S. Department of Energy Offjce of Uranium Enrichment and Assessment. 1982. Survey of United States Uranium Marketing Activity. Report No. DOE/NE-00131, U.S. Department of Energy, Office of Uranium Enrichment and Assessment, Washington, D.C.

U.S. Department of Energy. 1979. Statistical Data of the Uranium Industry. Report No. GJ0-100(79), U.S. Department of Energy, Grand Junction Area office, Grand Junction, Colorado.

U.S. Department of Energy. 1980. Statistical Oata of the Uranium Industry. Report No. GJ0-100(80), U.S. Department of Energy, Grand Junction Area Office, Grand Junction, Colorado.

U.S. Department of Energy. 1981. Statistical Data of the Uranium Industry. Report No. GJ0-100(81), U.S. Department of Energy, Grand Junction Area Office, Grand Junction, Colorado.

U.S. Department of Energy. 1982. Statistical Data of the Uranium Industry. Report No. GJ0-100(82), U.S. Department of Energy, Grand Junction Area Office, Grand Junction, Colorado.

U.S. Department of Energy. 1983. Statistical Oata of the Uranium Industry. Report No. GJ0-100(83), U.S. Department of Energy, Grand Junction Area Office, Grand Junction, Colorado.

U.S. Department of Energy, Energy Information Administration. 1977. Energy Information Administration Annual Report to Congress. Report No. DOE/EIA$0036(77) / 2$, U.S. Department of Energy, Energy Information Administration, Washington, D.C.

U.S. Department of Energy, Energy Information Administration. 1982. Investment in Exploration by the U.S. Uranium Industry. Report No. DOE/EIA-0362, U.S. Department of Energy, Energy Information Administration Washington, $0 . C$. 
U.S. Department of Energy, Energy Information Administration. 1983. 1982 Annual Energy Outlook. Report No. D0E/EIA-0383(32), U.S. Department of Energy, Energy Information Administration, Washington, D.C.

U.S. Department of the Interior, Bureau of Land Management. Arizona State Office. 1980. Wilderness Review Arizona, Intensive Inventory of Public Lands Administered by Bureau of Land Management. U.S. Department of Interior, Bureau of Land Management, Arizona State Office, Phoenix, Arizona.

U.S. Department of the Interior, Bureau of Land Management, Colorado State Office. 1980. Intensive Wilderness Inventory, Analys is of Public Comment and Final Wilderness Study Areas. U.S Department of the Interior, Bureau of Land Management, Colorado State Office, Denver, CoTorado.

U.S. Department of the Interior, Bureau of Land Management, New Mexico State Office. 1980. New Mexico Wilderness Study Area Decisions. U.S. Department of the Interior, Bureau of Land Management, New Mexico State Office, Santa Fe, New Mexico.

U.S. Department of the Interior, Bureau of Land Management, Ut ah State office. 1980. Utah Intensive Wilderness Inventory, Proposed Wilderness Study Areas. U.S. Department of the Interior, Bureau of Land Management, Utah State office, Salt Lake City, Utah.

U.S. Department of Interior, Bureau of Land Management, Wyoming State Office. 1981. Wyoming Wilderness Study Areas, A Final Inventory Report. U.S. Department of the Interior, Bureau of Land Management, Wyoming State Office, Cheyenne, Wyoming.

U.S. Department of Labor-Mine Safety and Health Administration, 1982, Uranium Mines Address Listing with Workers and Employee-hours, 3rd quarter, 1982, Health and Safety Analysis Center, Denver, Colorado.

U.S. Nuclear Regulatory Commission. 1980. Draft Environmental Impact Statement Related to the Operation of Bison Basin Project Petroleum, Inc. Report No. NUREG-0687, U.S Nuclear Regulatory Commission, Washington, D.C.

Weissbrod, R., and W. Barron. 1979. Geothermal Energy Market Study on the Atlantic Coastal Plain, A Review of Recent Energy Price Projections for Traditional Space Heating Fuel 1985-2000. Johns Hopkins University, Laurel, Maryland.

White, George Jr. 1978. "Uranium: Prices Steady at High Levels in '77". E\&MJ, March, 1979.

White, George Jr. 1979. "Uranium, Continued Price Stability Seen For 1979, as Supply and Demand Stay in Balance". E\&MJ, March 1979.

White, George Jr. 1980. "Uranium, Improving Supplies Weakens Price Structure". E\&MJ, March, 1980. 
White, George Jr. 1981. "Uranium, Supply-Demand Imbalance Leads to Sharp

Price Break and Intense Seller Competition". E\&MJ, March, 1981.

White, George Jr. 1982. "Uranium, Producer Purchases and Utility Sales Lead to Unstable Equilibrium". E\&MJ, March 1982.

White, George Jr. 1983. "Uranium, Prices Slump Under Inventory Selling But Firm Up at Year End". E\&MJ, March 1983.

White, J. L., et al. 1979. Energy from the West: Energy Resource Development Systems Report - Vol . 1: Introduction and General Soclal Controls, Report No. PB-299177. Science and Public Policy Program, University of Ok lahoma, Norman, OK lahoma.

Wright, John H. 1980. "Economic Geology of the Schwartzalder Mine," In Proceedings: Third Uranium Resource/Technology Seminar, March, 1980, Golden, Colorado, Colorado School of Mines Press, Golden, Cotorado. 


\section{APPENDIX A}

GLOSSARY OF MINING TERMS 
APPENDIX A

GLOSSARY OF MINING TERMS

back

cribbing

cross-cut

drift

muck

raise

roof

roof bolt

room and pillar method

sand $f i 11$

adit

shaft collar

sill
The roof or upper part of any underground mining cavity.

The construction of cribs, or timbers laid at right angles to each other, sometimes filled with earth, as a roof support or as a support for machinery. The close setting of timber supports when shaft sinking through loose ground. The timber is usually square or rectangular and practically no ground is exposed. The method is also used for constructing ore chutes.

A horizontal opening driven across the course of vein or, in general, normal to the direction of main workings.

A horizontal opening in or near an ore body and parallel to the course of the vein or the long dimension of the ore body.

Rock or ore broken in the process of mining.

A vertical or inclined opening driven upward from a level to connect with the level above, or to explore the ground for a limited distance above one level.

The ceiling of any underground excavation. Same as the "back."

Long steel bolts driven into walls or roof of underground excavations to strengthen the pinning of rock strata. They are expanded by means of a wedge which opens a sleeve surrounding it.

In coal and metal mining, supporting the roof by pillars left at regular or irregular intervals.

Hydraulic or pnematic filling, stowing. Use of sand conveyed underground by water or air to support cavities left by extraction of ore.

A timber or steel frame for supporting the sides of an excavation, shaft or tunnel.

Nearly horizontal passage from the surface.

Supporting framework at top of shaft from which linings may be hung. The term applies to the timber, steel, or concrete around the mount or top of a shaft.

The floor of an opening or passage in a mine. 
Glossary of Mining Terms (contd)

slusher A machine used for loading coal or rock by pulling an openbottomed scoop back and forth between the face and the loading point by means of ropes, sheaves, and a multiple drum hoist.

square set

stoping

A method of stoping in which the walls and back of the excavation are supported by regular framed timbers forming a skelton enclosing a series of connected, hollow, rectangular prisms in the space fomerly occupied by the excavated ore and providing continuous lines of support in three directions at right angles to each other. The ore is excavated in small, rectangular blocks just large enough to provide room for standing a set of timber.

stope

Commonly applied to the extraction of ore, but does not include the ore removed in sinking shafts and in driving levels, drifts, and other development openings.

Source: New Mexico Energy and Minerals Department. 
APPENDIX B

PRODUCTIVITY FACTOR CALCULATION 
APPENDIX B

PRODUCTIVITY FACTOR CALCULATION

The productivity factor is calculated as follows:

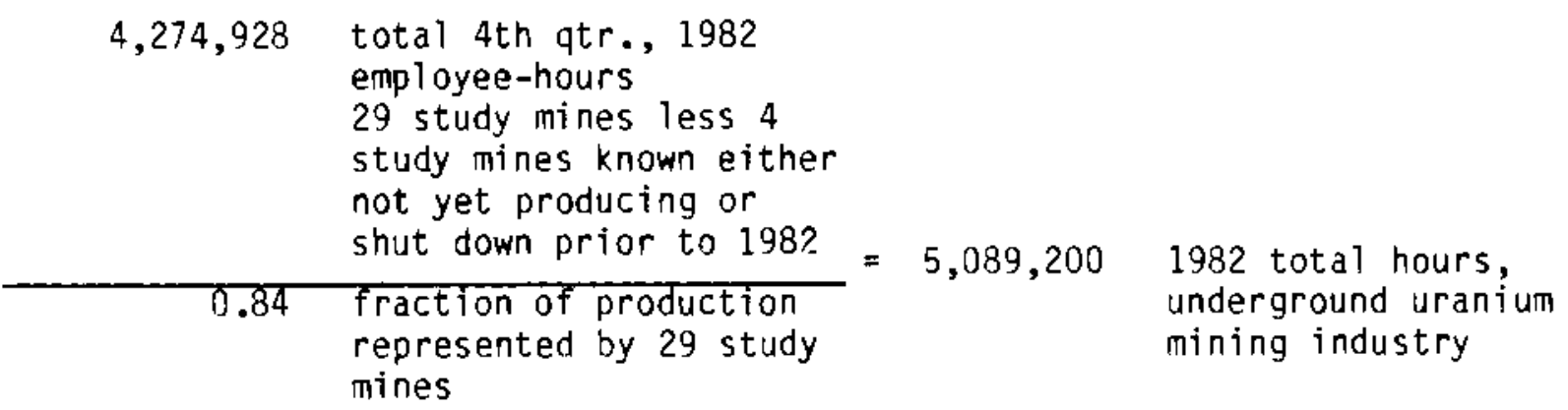

$5,089,200$ hrs $=2545$ 1982 total FTE workers, 2000 hrs equalting one FtE worker underground uranium mining industry (a)

62001982 total tons $U_{3} 0_{8}$ for underground mining industry

2545 FTE workers $=2.44$

tons $\mathrm{U}_{3} \mathrm{O}_{\mathrm{g}}$ per $\mathrm{FTE}$ worker per year, underground uranium mining industry.

(a) The actual total numbers of underground uranium "miners" (1982 average employment level) as reported by DOE (1983) is 1275. This much lower, average number is probably due to the "substantial layoffs which occurred late in 1982" as noted by the DOE. Additionally, the DOE breaks down the total underground employment (2150) into "miners" (1275) and "service and support" (875). The MSHA (1982) data does not make such a breakdown distinction in its employee-hours, "workers," listing. 
APPENDIX C

STUDY MINE DATA SHEETS 


\section{APPENDIX C}

MINE NAME: Hacks Canyon \#1 and $\# 2$

COMPANY: Energy Fuels Nuclear, Inc.

LOCATION: T37N, R5W, N1/2 Sec. 26, Mohave County, Arizona

OPERATING STATUS: Active as of $2 / 83$, although Energy Fue is Nuclear suspended milling operations on 1/21/83 (E \& MJ 1983).

DESIGNATED MINE CAPACITY: $300-500$ tons ore per day (a)

ANNUAL PRODUCTION: N.A.(b)

CUMULATIVE PRODUCTION: 1950, '52, '53, '54, '64 - Hacks \#1 (?) produced 1,329 tons ore at $0.18 \% \cup_{3} 0_{8}$ (Scarborough 1981).

RESERVES: There is a possible 500,000 tons of uranium-copper ore or about 3 million pounds $\mathrm{U}_{3} \mathrm{O}_{8}$ (new discovery as announced by Energy Fuels Nuclear, December, 1980, for Hacks Canyon \#2) (Scarborough 1981).

DEPTH: N.A.

GRADE: Hacks Canyon \#1: $\quad 0.009-1.798 \% \mathrm{U}_{3} 0_{8}$ (Scarborough 1981)

Hacks Canyon \#2: $\quad 0.30 \% \mathrm{U}_{3} 0_{8}^{8}$ (Scarborough 1981)

- could bé as high as $1.0 \% \mathrm{U}_{3} \mathrm{O}_{8}$ (a)

AGE OF MINE: first ore shipment in 1981 from Hacks \#2. (a)

OTHER COMMENTS: - Energy Fuels Nuclear has a mill in 81 anding, utah, which opened in 1980. It is a $2000 \mathrm{st} / \mathrm{d}$ operation. Mill feed is from the two Hacks Canyon Mines and other toll milling sources (E \& MJ 1983)

- R. B. Scarborough says that Energy Fuels Nuclear is a privately owned company by a group of individuals. Its president, Bob Adams, died recently and the Company has since slowed down somewhat. He says that Energy fuels Nuclear is a very secretive company as to their exploration techniques and deposit statistics. Energy Fuels has the strip of county north of the Grand Canyon fairly tied

\footnotetext{
(a) Information from R. B. Scarborough, Arizona Bureau of Geology and Mineral Technology, Tucson, Arizona.

(b) N.A. = not available
} 
MINE NAME: Pigeon Mine

COMPANY: Energy Fueis Nuclear, Inc.

LOCATION: T38N, R2W, N1/2 Sec. 5, Coconino County, Arizona

OPERATING STATUS: As of $2 / 83$ the mine is still under construction. Production and ventilation shafts are completed, the mine is being readied for future production ( $E \& M J$ 1983).

DESIGNATED MINE CAPACITY: N.A.

ANNUAL PRODUCTION: none

CUMULATIVE PRODUCTION: none

RESERVES: N.A.

DEPTH: N.A.

GRADE: N.A.

AGE OF MINE: not yet producing $(a)$

OTHER COMMENTS: The Pigeon Mine has a 1400 fopt vertical shaft. A second shaft is now being drilled. (a)

(a) Information from Bill Chenoweth, U.S. Department of Energy, Grand lunction, Colorado. 
$\because \quad$ MINE NAME: Kanab North

COMPANY: Energy Fuels Nuclear, Inc.

LOCATION: T38N, R3W, SW1/4 Sec. 17, Mohave County, Arizona

OPERATING STATUS: - Construction bids have been solicited, but no

- The company recently got permission from the BL.M to begin sinking a shaft. (b)

DESIGNATED MINE CAPACITY: N.A.

ANNUAL PRODUCTION: none

CUMULATIVE PRODUCTION: none

RESERVES: Unknown by Energy Fuels Nuclear. They are sinking the shaft from limited drill data and assumptions that the ore body exists from their geologic model. (b)

DEPTH: N.A.

GRADE: N.A.

AGE OF MINE: not yet producing $(b)$

OTHER COMMENTS:

\footnotetext{
(a) Information from W. I. Enderlin, Battelle Pacific Northwest Laboratories, Richland, Washington.

(b) Information from Bill Chenoweth, U.S. Department of Energy, Junction, colorado.
} 
MINE NAME: Sunday

COMPANY: Union Carbide Corporation

LOCATION: T44N, R18W, SEl/4 Sec. 13, San Miguel County, Colorado

OPERATING STATUS: active as of $11 / 82$ (U.S. Dept. of Labor 1982)

DESIGNATED MINE CAPACITY: N.A.

ANNUAL PRODUCTION: 1980: underground mines feeding Uravan mill produced $100,000-500,000$ tons ore (E\& MJ 1981).

CUMULATIVE PRODUCTION: N.A.

RESERVES: N.A.

DEPTH: N.A.

GRADE: N.A.

AGE OF MINE: N.A.

OTHER COMMENTS: 
MINE NAME: King Solomon

COMPANY: Union Carbide Corporation

LOCATION: T48N, R17W, NW1/4 Sec. 19, Montrose County, Colorado

OPERATING STATUS: active as of $11 / 82$ (U.S. Dept. of Labor 1982).

DESIGNATED MINE CAPACITY: N.A.

ANNUAL PRODUCTION: 1980: underground mines feeding Uravan mill produced $100,000-500,000$ tons ore (E\& M 1981).

CUMULATIVE PRODUCTION: N.A.

RESERVES: N.A.

DEPTH: N.A.

GRADE: N.A.

AGE OF MINE: N.A.

OTHER COMMENTS: 
MINE NAME: Deremo-Snyder

COMPANY: Union Carbide Corporation

LOCATIDN: T42N, R2OW, 5 1/2 Sec. 2, San Miguel County, Colorado

OPERATING STATUS: active as of 11/82 (U.S. Dept. of Labor 1982).

OESIGNATEO MINE CAPACITY: N.A.

ANNUAL PROOUCTION: 1980: underground mines feeding Uravan mill produced 100,000-500,000 tons ore (E \& MJ 1981).

CUMULATIVE PRODUCTION: N.A.

RESERVES: N.A.

DEPTH: N.A.

GRADE: N.A.

AGE OF MINE: N.A.

OTHER COMMENTS: 
MINE NAME: Mt. Taylor

COMPANY: Gulf Mineral Resources

LOCATION: T13N, R8W, SE 1/4 Section 24, Cibola County, New Mexico

OPERATING STATUS: Placed on standby status in November 1982, with support facilities being maintained (Gulf 0il Corp. 1982).

DESIGNED MINE CAPACITY: Expected production of 4,000-4,500 tons/day or 1.42 million tons ore/year (Perkins 1979).

ANNUAL PRODUCTION: N.A.

CUMULATIVE PRODUCTION: N.A.

RESERVES: $125 \mathrm{million} 1 \mathrm{~b} \mathrm{U}_{3} \mathrm{O}_{8}$ (a)

DEPTH: - 3,100 ft (Energy and Minerals Dept 1982)

- $\sim 3,300 \mathrm{ft}$ (Perkins 1979)

GRADE: Average grade of $0.25-0.30 \% \mathrm{U}_{3} \mathrm{O}_{8}$ (a)

AGE OF MINE: Mining scheduled to begin 1979, with 20 year expected life (Perkins 1979).

OTHER COMMENTS: - Were producjug until late 1982; toll milling at KerrMcGee mill. af

- There is reported significant quantities of Molybdenum and Vanadium in the ore. Rock face temperatures are expected to reach $130^{\circ} \mathrm{F}$, requiring air conditioning and worker's to possibly wear ice vests (Perkins 1979).

- Gulf hasn't sold any $U_{3} O_{8}$ to date from Mt. Taylor. The company has a $\$ 419$ million investment in the Mt. Taylor mine complex as of $12 / 31 / 82$. Development will resume when uranium demand improves and market prices return to economic levels (Gulf 0 il Corp. 1982).

(a) Information from C. Wentz, Uranium Specialist, Energy and Minerals Department, Santa Fe, New Mexico. 
MINE NAME: 01d Church Rock

COMPANY: UNC, Resources, Inc.

LOCATION: T16N, Rl6W, N 1/2 Section 17, MCKinley County, New Mexico

OPERATING STATUS: Placed on standby in April, 1982. Decision made to allow the mine to flood (UNC Resources, Inc. 1982)

DESIGNED MINE CAPACITY: N.A.

ANNUAL PRODUCTION: $1982-0.6$ million lb $\mathrm{U}_{3} \mathrm{O}_{8}$ produced in concentrate from UNC's Church Rock Mines (Old Church Rock and Church Rock NE) (UNC Resources, Inc. 1982)

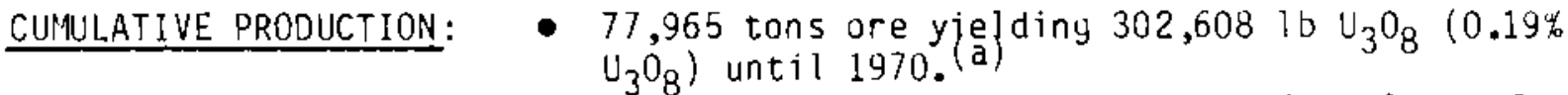

- U.S. Department of Energy production class of 200,000 to 2 million ib $\mathrm{U}_{3} \mathrm{O}_{8}$.

RESERVES: $33.6 \mathrm{million}$ tons ore material containing $57.3 \mathrm{million} 1 \mathrm{~b} \mathrm{~J}_{3} \mathrm{U}_{8}$ recoverable by conventional mining methods (as of 12/31/82).

Reserve figure includes the following mines:

2 Church Rock Mines (on standby)

3 Ambrosia Lake Mines (all on standby status)

St. Anthony Mines, Laguna area (on standby) (UNC Resources, Inc. 1982)

DEPTH: Shaft $\# 1-862 \mathrm{ft}$, Shaft $\# 2-800-900 \mathrm{ft}(\mathrm{a})$

GRADE: Figured to be about $0.10 \%$ (calculated from reserves data in annual report) (UNC Resources, Inc. 1982)

AGE OF MINE: Years of operation: $1960-62,1976-77$, and 1979-82(a)

OTHER COMMENTS: Previously mined in the Dakota and Westwater stratigraphic units of the Morrison Formation from a vertical shaft by Phillips Petroleum Company in 1960-1962 (Perkins 1979).

(a) Information from V. T. McLemore, Uranium Geologist, New Mexico Bureau of Mines and Minerals Technology, Socorro, New Mexico. 
MINE NAME: Church Rock Northeast

COMPANY: UNC Resources, Inc.

LOCATION: TI7N, R16W, Section 35, McKinley County, New Mexico

OPERATING STATUS: Placed on standby in April, 1982. Decision made to allow the mine to flood (UNC Resources, Inc. 1982)

DESIGNED MINE CAPACITY: Up to 3,000 tons/day (Perkins 1979)

ANNUAL PRODUCTION: $1982-0.6$ million $1 \mathrm{~b} . \mathrm{U}_{3} \mathrm{O}_{8}$ produced in concentrate from UNC's Church Rock Mines (old Church Rock and Church Rock NE) (UNC Resources, Inc. 1982)

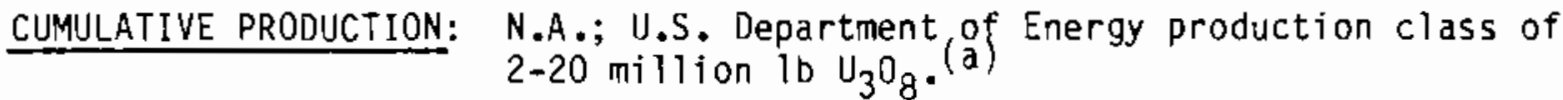

RESERVES: - 33.6 million tons ore material containing 57.3 million $1 \mathrm{~b} \mathrm{U}_{3} \mathrm{O}_{8}$

recoverable by conventional mining methods (as of $12 / 31 / 82$ ).

Reserve figure includes the following mines:

2 Church Rock Mines (on standby)

3 Ambrosia Lake Mines (all on standby status)

St. Anthony Mines, Laguna area (on standby) (UNC Resources, Inc. 1982)

- 20 million tons ore $\left(60\right.$ million $\left.16 U_{3} 0_{8}\right)$ (Perkins 1979)

- 15 mitition $1 \mathrm{~b} \mathrm{U}_{3} \mathrm{O}_{8}$ at $0.15 \% \mathrm{U}_{3} \mathrm{O}_{8}$ estimated in 1969 . (a)

DEPTH: - 1,700 ft (Energy and Minerals Dept. 1982)

GRADE: Average 0.15\% U U $0_{8}$ (Perkins 1979).

AGE OF MINE: Began production in $1972^{(a)}$

OTHER COMMENTS: The mine has two vertical shafts and five exhaust fans with an air discharge of 667,500 (ACFM) (Perkins 1979).

(a) Information from V. T. McLemore, Uranium Geologist, New Mexico Bureau of Mines and Minerals Technology, Socorro, New Mexico. 
MINE NAME: Church Rock \#1

COMPANY: Kerr-MCGee Corporation

LOCATION: TI7N, Ri6W, Section 35, MCKinley County, New Mexico

OPERATING STATUS: Producing as of $7 / 16 / 82$ (Energy and Minerals Dept. 1982)

DESIGNED MINE CAPACITY: Production goal of 1,000 tons per day (Perkins 1979)

ANNUAL PROOUCTION: 1982: $1,170,473$ tons of company mined ore were processed at the Ambrasia Lake mill at an average grade of $0.174 \%$

(ore from the Ambrosia Lake and Church Rock Mines)

(Kerr-McGee Corp. 1982).

CUMULATIVE PRODUCTION: N.A.; U.S. Department of Energy production class of $2-20$ million $1 \mathrm{~b} \mathrm{U}_{3} \mathrm{O}_{8}$.

RESERVES: At 6 foot $/ 0.10 \%$ cut off, Church Rock ore body (combined Church Rock \#1 and Church Rock East Mines) - 2,345,000 tons ore containing $10,168,0001 \mathrm{~b} \mathrm{U}_{3} \mathrm{O}_{8}$. These reserves "relate on ly to those estimated quantities of uranium - bearing material which are available to mines actively producing ... and which are believed capable of being profitably mined and sold under present technology, regulatory and economic conditions" (Kerr-McGee Corp. 1982).

DEPTH: - Maximum depth of $1,851 \mathrm{ft}$ (Perkins 1979)

- 1,673-1,755 ft deep (Energy and Minerals Dept. 1982)

GRADE: $\quad 0.22 \% \mathrm{U}_{3} \mathrm{O}_{8}$ (Kerr-McGee Corp. 1982)

AGE OF MINE: - Began production 1973 or 1974 (D)

- Another communication says 1976 (a)

OTHER COMMENTS: - The mine has one vertical shaft and four exhaust fans with an air discharge of 392,000 (ACFM) (Perkins 1979).

- In 1978, the mine waste bench was over 30 acres. Mined rock of less than $0.03-0.05 \% \mathrm{U}_{3} \mathrm{O}_{8}$ goes to the waste bench. Heat output of the mine is about 350,000 BTU's per minute. The initial developinent cost has been $\$ 15$ million (Perkins 1979).

(a) Information from V. T. McLemore, Uranium Geologist, New Mexico Bureau of Mines and Minerals Technology, Socorro, New lexico.

(b) Information from $C$. Wentz, Ur anium Specialist, Energy and Minerais Department, Santa Fe, New lexico. 
MINE NAME: Church Rock East

COMPANY: Kerr-McGee Corporation

LOCATION: TI7N, Rl6W, Section 36, MCKinley County, New Mexico

OPERATING STATUS: Producing as of $7 / 16 / 82$ (Energy and Minerals Dept. 1982)

DESIGNED MINE CAPACITY: Originally 500 tons/day estimated; 1979 - production increased to 1500 tons/day (Perkins 1979).

ANNUAL PRODUCTION: 1982: 1,170,473 tons of company mined ore were processed at the Ambrosia Lake mill at an average grade of $0.174 \%$ (ore from the Ambrosia Lake and Church Rock Mines) (KerrMcGee Corp. 1982)

CUMULATIVE PRODUCTION: N.A.; U.S. Department of Energy production class of $200,000-2$ million $1 b U_{3} 0_{8}(a)$

RESERVES: At 6 foot $/ 0.10 \%$ cut of $f$, Church Rock ore body (combined church Rock \#1 and Church Rock East Mines) - 2,345,000 tons ore containing $10,168,000$ 1b $U_{3} \mathrm{O}_{8}$. These reserves "relate only to those estimated quantities of uranium - bearing material which are available to mines actively producing .... and which are believed capable of being profitably mined and sold under present technology, regulatory and economic conditions" (Kerr-McGee Corp. 1982).

DEPTH: $\bullet 1,545 \mathrm{ft}$,expected depth (Perkins 1979)

- $1,635 \mathrm{ft}^{(\mathrm{a})}$

GRADE: $\quad 0.22 \% \mathrm{U}_{3} \mathrm{O}_{8}$ (Kerr-MCGee Corp. 1982)

AGE OF MINE: Began production in $1979^{(b)}$

OTHER COMMENTS: In 1979, the east vent hole was enlarged to be used for hoisting, decreasing the haulage distance for ore mined from the east ore body (Perkins 1979).

(a) Information from V. T. McLemore, Uranium Geologist, New lexico Bureau of inines and Minerals Technology, Socorro, New Mexico.

(b) In formation from C. Wentz, IJanium Specialist, Energy and Minerals Department, Santa Fe, New Mexico. 
MINE NAME: Kerr-McGee Section 30E

COMPANY: Kerr-McGee Corporation

LOCATION: TI4N, R9W, E 1/2, Section 30, MCKinley County, New Mexico

OPERATING STATUS: Active as of $2 / 83$, but at reduced production levels. (a)

DESIGNED MINE CAPACITY: N.A.

ANNUAL PRODUCTION: 1982 reported company mined ore processed at Ambrosia Lake mill was $1,170,473$ tons (includes all Ambrosia Lake Mines and two Church Rock Mines) (Kerr-McGee Corp. 1982).

CUMULATIVE PROOUCTION: - The Ambrosia Lake mill, in 24 years, has processed 33 million tons of ore containing 125 million $1 \mathrm{~b}$ $\mathrm{U}_{3} \mathrm{O}_{8}$ (Kerr-McGee Corp. 1982).

- U.S. Department of Energy production class of 2-20 million 1b $\mathrm{U}_{3} \mathrm{O}_{8}$; (b)

- 2,855,164 tons ore yjelding $15,064,956$ 1b $\mathrm{U}_{3} \mathrm{O}_{8}$
$(0.26 \%)$ until 1970 . (b)

RESERVES: At 5 operating mines in 1982 (Section 19, 30, 30 W, 35, 36) $3,925,000$ tons ore containing $17,105,000$ lb U $_{3} 0_{8}$ (Kerr-McGee Corp. 1982).

DEPTH: $\quad 656 \mathrm{ft}$ (Energy and Minerals Dept. 1982)

- $750 \mathrm{ft}(\mathrm{b})$

GRADE: $0.22 \% \mathrm{U}_{3} \mathrm{O}_{8}$ (average for five mines this study) (Kerr-McGee Corp. 1982)

AGE OF MINE: Began production in $1959^{(b)}$

OTHER COMMENTS: - The mine has one vertical shaft, and 11 exhaust fans with air discharge of 348,500 (ACFM) (Perkins 1979).

- At the Kerr-McGee's Ambrosia Lake properties, approximately $12 \%$ of the $\mathrm{U}_{3} \mathrm{O}_{8}$ is held by mining claims on Federal lands which are owned or leased, while the remainder of the $\mathrm{J}_{3} \mathrm{O}_{8}$ is heid under mining leases on fee lands (Kerr-McGee Corp. 1982).

(a) Information from T. G. Brough, Ph.D., Environmental Scientist, Radiation Protection Bureau, Environmental Improvement Division, Milan, New Mexico.

(b) Information from V. T. McLemore, Uranium Geologist, New Mexico Bureau of Mines and Minerals Technology, Socorro, New Mexico. 
MINE NAME: Kerr-McGee Section 30W

COMPANY: Kerr-McGee Corporation

LOCATION: T14N, R9W, W 1/2, Section 30, McKinley County, New Mexico

OPERATING STATUS: Active as of $2 / 83$, but at reduced production leveis $(a)$

DESIGNED MINE CAPACITY: N.A.

ANNUAL PRODUCTION: 1982 reported company mined ore processed at Ambrosia Lake mill was $1,170,473$ tons (includes all Ambrosia Lake Mines and two Church Rock Mines) (Kerr-McGee Corp. 1982).

CUMULATIVE PRODUCTION: - The Ambrosia Lake mill, in 24 years, has processed $33 \mathrm{million}$ tons of ore containing $125 \mathrm{million} 1 \mathrm{~b}$ $\mathrm{U}_{3} \mathrm{O}_{8}$ (Kerr-McGee Corp. 1982).

- U.S. Department of Energy production class of 2-20 million $1 \mathrm{~b} \mathrm{U}_{3} \mathrm{O}_{8}$; (b)

- 68,895 tons (pre yielding 282,714 lb $\mathrm{U}_{3} 0_{9}(0.21 \%)$

RESERVES: At 5 operating mines in 1982 (Section 19, 30, 30W, 35, 36) $3,925,000$ tons ore containing $17,105,0001 \mathrm{~b} \cup_{3} 0_{8}$ (Kerr-McGee Corp. 1982).

DEPTH: $-701-740 \mathrm{ft}$ (Energy and Minerals Dept. 1982)

GRADE: $0.22 \% \mathrm{U}_{3} \mathrm{O}_{8}$ (average for five mines this study) (Kerr-McGee Corp. 1982) AGE OF MINE: Began production in 1969 (b)

OTHER COMMENTS: - Vertical shaft, four exhaust fans with air discharge of 265,000 (ACFM) (Perkins 1979).

- At the Kerr-McGee's Ambrosia Lake properties, approximately $12 \%$ of the $\mathrm{U}_{3} \mathrm{O}_{8}$ is held by mining claims on Federal lands which are owned or leased, while the remainder of the $\mathrm{U}_{3} \mathrm{O}_{8}$ is held under mining leases on fee lands (Kerr-McGee Corp. 1982).

(a) Information from T. G. Brough, Ph.D., Environmental Scientist, Radiation Protection Bureau, Environmental Improvement Division, Milan, New Mexico.

(b) Information from V. T. McLemore, Uranium Geologist, New Mexico Bureau of Mines and Minerals Technology, Socorro, New Mexico. 
MINE NAME: Kerr-MCGee Section 19

COMPANY: Kerr-MCGee Corporation

LOCATION: Tl4N, R9W, Section 19, Mckinley County, New Mexico

OPERATING STATUS: Producing as of 7/16/82 (Energy and Minerals Dept. 1982)

DESIGNED MINE CAPACITY: N.A.

ANNUAL PRODUCTION: 1982 reported company mined ore processed at Ambrosia Lake mill was $1,170,473$ tons (includes all Ambrosia Lake Mines and two Church Rock Mines (Kerr-McGee Corp. 1982)

CUMULATIVE PRODUCTION: - The Ambrosia Lake mill, in 24 years, has processed 33 million tons of ore containing 125 million $1 \mathrm{~b}$ $\mathrm{U}_{3} \mathrm{O}_{8}$ (Kerr-McGee Corp. 1982).

- U.S. Department of Energy production class of 2-20 million $1 \mathrm{~b} \mathrm{U}_{3} \mathrm{O}_{8}$, (a)

RESERVES: At 5 operating mines in 1982 (Section $19,30,30 \mathrm{~W}, 35,36$ ) $3,925,000$ tons ore containing $17,105,0001 \mathrm{~b} \cup_{3} 0_{8}$ (Kerr-McGee Corp. 1982)

DEPTH: $\quad$ - 640-7U5 ft (Energy and Minerals Dept. 1982)

GRADE: $0.22 \% \mathrm{U}_{3} \mathrm{O}_{8}$ (average for five mines this study) (Kerr-l/cGee Corp. 1982) AGE OF MINE: Began production $1976^{(a)}$

OTHER COMMENTS: - The mine has six exhaust fans, with air discharge of 205,000 (ACFM) (Perkins 1979).

- At the Kerr-McGee's Ambrosia Lake properties, approximately $12 \%$ of the $\mathrm{U}_{3} \mathrm{O}_{8}$ is held by mining claims on Federal 1 ands which are owned or leased, while the remainder of the $\mathrm{U}_{3} \mathrm{D}_{8}$ is held under mining leases on fee lands (Kerr-McGee Corp. 1982).

(a) Information from V. T. McLemore, Uranium Geologist, New Mexico Bureau of Mines and Minerals Technology, Socorro, New lexico. 
MINE NAME: Kerr-McGee Section 35

COMPANY: Kerr-MeGee Corporation

LOCATION: T14N, R9W, Section 35, McKinley County, New Mexico

OPERATING STATUS: ACtive as of $2 / 83$, but at reduced production levels(a)

DESIGNED MINE CAPACITY: N.A.

ANNUAL PRODUCTION: 1982 reported company mined ore processed at Ambrosia Lake mill was $1,170,473$ tons (includes all Ambrosia Lake Mines and two Church Rock Mines) (Kerr-McGee Corp. 1982).

CUMULATIVE PRODUCTION: - The Ambrosia Lake mill, in 24 years, has processed $33 \mathrm{million}$ tons of ore containing $125 \mathrm{million}$ lb $\mathrm{U}_{3} \mathrm{O}_{8}$ (Kerr-McGee Corp. 1982).

- U.S. Department of Energy production class of 2-20 million $1 \mathrm{~b} \mathrm{U}_{3} \mathrm{O}_{8}$, (b)

RESERVES: At 5 operating mines in 1982 (Section 19, 30, 300, 35, 36) $3,925,000$ tons ore containing $17,105,0001 \mathrm{~b} \mathrm{U}_{3} \mathrm{O}_{8}$ (Kerr-McGee Corp. 1982).

OEPTH: $\quad$ - $1,186-1,335 \mathrm{ft}$ (Energy and Minerals Dept. 1982)

GRADE: $0.22 \% \mathrm{U}_{3} 0_{8}$ (average for five mines this study) (Kerr-McGee Corp. 1982). AGE OF MINE: Began production $1971^{\text {(b) }}$

OTHER COMMENTS: - The mine has one vertical shaft, and six exhaust fans with air discharge of 414,000 (ACFM) (Perkins 1979).

- At the Kerr-McGee's Ambrosia Lake properties, approximately $12 \%$ of the $\mathrm{U}_{3} \mathrm{O}_{8}$ is held by mining claims on Federal lands which are owned or leased, while the remainder of the $\mathrm{U}_{3} \mathrm{O}_{8}$ is held under mining leases on fee lands (Kerr-McGee Corp 1982). This mine was originally called the Elizabeth Mine.

\footnotetext{
(a) Information from T. G. Brough, Ph.D., Environmental Scientist, Radiation Protection Bureau, Environmental Improvement Division, Milan, New Mexico.

(b) Information from V. T. McLemore, Uranium Geologist, New Mexico Bureau of Mines and Minerals Technology, Socorro, New Mexico.
} 
MINE NAME: Kerr-McGee Section 36

COMPANY: Kerr-McGee Corporation

LOCATION: T14N, R9w, Section 36, MCKinley County, New Mexico

OPERATING STATUS: Active as of $2 / 83$, but at reduced production levels $(a)$

DESIGNED MINE CAPACITY: N.A.

ANNUAL PRODUCTION: 1982 reported company mined ore processed at Ambrosia Lake mill was $1,170,473$ tons (includes all Ambrosia Lake Mines and two Church Rock Mines) (Kerr-McGee Corp 1982).

CUMULATIVE PRODUCTION: - The Ambrosia Lake mill, in 24 years, has processed 33 million tons of ore containing 125 million lb $\mathrm{U}_{3} \mathrm{O}_{8}$ (Kerr-McGee Corp. 1982).

- U.S. Department of Energy production class of 2-20 million $1 \mathrm{~b} \mathrm{U}_{3} \mathrm{O}_{8}$ 745,074 tons ore yielding $6,046,780$ lb $\mathrm{U}_{3} \mathrm{O}_{8}$
$(0.41 \%)$ until 1970 . (b)

RESERVES: At 5 operating mines in 1982 (Section $19,30,30 \mathrm{~W}, 35,36$ ) $3,925,000$ tons ore containing $17,105,0001 \mathrm{~b} \mathrm{U}_{3} 0_{8}$ (Kerr-McGee Corp. 1982)

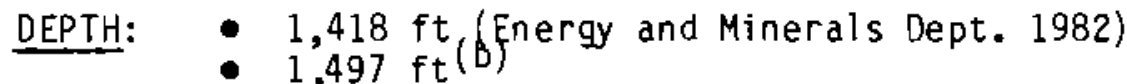

GRADE: $0.22 \% \mathrm{U}_{3} \mathrm{O}_{8}$ (average for five mines this study) (Kerr-McGee Corp. 1982). AGE OF MINE: Began production $1960^{(b)}$

OTHER COMMENTS: - The mine has one vertical shaft, and four exhaust fans with air discharge of 190,900 (ACFM) (Perkins 1979).

- At the Kerr-McGee's Ambrosia Lake properties, approximately $12 \%$ of the $\mathrm{U}_{3} \mathrm{O}_{8}$ is held by mining claims on Federal 1 ands which are owned or leased, while the remainder of the $\mathrm{U}_{3} \mathrm{O}_{8}$ is held under mining leases on fee lands (Kerr-McGee Corp. 1982). This mine was originally called the Cliffside Mine.

(a) Information from T. G. Brough, Ph.D., Environmental Scientist, Radiation Protection Bureau, Environmental Improvement Division, Milan, New Mexico.

(b) Information from V. T. McLemore, Uranium Geologist, New Mexico Bureau of Mines and Minerals Technology, Socorro, New Mexico.

$$
\text { C. } 16
$$


MINE NAME: Homestake Section 23

COMPANY: Homestake Mining Company

LOCATION: TI4N, R1OW, Section 23, McKinley County, New Mexico

OPERATING STATUS: ACtive as of $2 / 83$, but at reduced production levels $(a)$

DESIGNED MINE CAPACITY: N.A.

ANNUAL PROOUCTION: - 1982 - ore from Grants Mines declined to 240,002 tons from 377,537 tons ore in 1981 . $U_{3} 0_{8}$ produced equaled $1,252,427$ lb (Homestake Mining Co. 1982).

- Most of the 1982 ore probably cane from the Section 23 Mine, since Section 13, 15, 32 Mines were closed as of $12 / 31 / 81$ and Section 25 closed since 1/1/82 (Energy and Minerals Dept. 1982). Homestake states that the higher grade areas of Section 23 Mine are now being processed.

CUMULATIVE PRODUCTION: - 2,528,797 tons ore yjelding $9,679,773$ lb $\mathrm{U}_{3} 0_{8}$
$(0.19 \%)$ until 1970 . (b)

- U. S. Department of Energy production class of $2-20$ million $1 \mathrm{~b} \mathrm{U}_{3} \mathrm{O}_{8}$.

RESERVES: Of the New Mexico Mines (stated as three underground mines, which includes Section 23) as of $12 / 31 / 82$, aggregated $2,150,000$ tons ore (proven and probable) (Homestake Mining Co. 1982).

DEPTH: $\quad \bullet 651-783 \mathrm{ft}$ (Energy and Minerals Dept. 1982)

GRADE: $0.169 \% \mathrm{U}_{3} \mathrm{O}_{8}$ (Homestake Mining Co. 1982)

AGE OF MINE: Began operation 1959 (b)

OTHER COMMENTS: The Homestake Grants operations consist of a mill and three underground mines, only one of which is now operating

(Section 23). Mineral properties consist of about 3,840 acres throughout the Ambrosia lake district.

of this total, 2,560 acres are leased from a railroad company, while 1,280 acres are leased from the state of New Mexico. The numerous uranium ore bodies range from 500-800 ft deep, a few to $100 \mathrm{ft}$ wide, up to several $100 \mathrm{ft} 10 \mathrm{ng}$ and 5-35 $\mathrm{ft}$ thick (Homestake Mining Co. 1982).

(a) Information from T. G. Brough, Ph.D., Environmental Scientist, Radiation Protection Bureau, Environmental Improvement Division, Milan, New Mexico.

(b) Information from V. T. McLemore, Uranium Geologist, New Mexico Bureau of Mines and Minerals Technology, Socorro, New lexico. 
MINE NAME: Homestake Section 25

COMPANY: Homestake Mining Company

LOCATION: T14N, R10W, Section 25, MCKinley County, New Mexico

OPERATING STATUS: - Closed since $1 / 1 / 82$ (Energy and Minerals Dept. 1982)

- Still clpsed as of $2 / 83$ with vent fans running as needed $(a)$

DESIGNED MINE CAPACITY: N.A.

ANNUAL PRODUCTION: 1982 - ore from Grants Mines declined to 240,002 tons from 377,537 tons ore 1981 (Homestake Mining Co. 1982). Since the Section 25 Mine was closed during 1982, probably only a very small percentage of this 1982 total represent the Section 25 Mine.

CUMULATIVE PRODUCTION:

- 1,791,048 tons ore yjelding 6,444,889 $10 \mathrm{U}_{3} 0_{8}$

- U.S. Department of Enepgy production class of 2-20 million ib $\mathrm{U}_{3} \mathrm{O}_{8}$.

RESERVES: Of New Mexico Mines (stated as three underground mines) as of 12/31/82 aggregated, $2,150,000$ tones ore (proven and probable) (Homestake Mining Co. 1982).

DEPTH: $\quad$ - $842-801 \mathrm{ft}$ ft (Energy and Minerals Dept. 1982)

GRADE: $0.169 \% \mathrm{U}_{3} \mathrm{O}_{8}$ (Homestake Mining Co. 1982)

AGE OF MINE: Began operation $1959^{(b)}$

OTHER COMMENTS: The Homestake Grants operations consist of a mill and three underground mines, only one of which is now operating (Section 23). Mineral properties consist of about 3,840 acres throughout the Ambrosia lake district. Of this total, 2,560 acres are leased from a railroad company, while 1,280 acres are leased from the state of New Mexico. The numerous uranium ore bodies range from 500-800 ft deep, a few to $100 \mathrm{ft}$ wide, up to several $100 \mathrm{ft}$ long and 5-35 $\mathrm{ft}$ thick (Homestake Mining Co. 1982).

(a) Information from T. G. Brough, Ph.D., Environmental Scientist, Radiation Protection Bureau, Environmental Improvement Division, Milan, New Mexico.

(b) Information from V. T. McLemore, Uranium Geologist, New Mexico Bureau of Mines and Minerals Technology, Socorro, New Mexico. 
MINE NAME: Nose Rock

COMPANY: Phillips Petroleum Company

LOCATION: T19N, R11W, Section 31, McKinley County, New Mexico

OPERATING STATUS: - Placed on standby in 1981 (Phillips Petroleum Co. 1982)

- Still inactive as of $2 / 83$ (a)

DESIGNED MINE CAPACITY: - Expected production of 2,100 tons ore/day (Perkins 1979)

- Expected full production of 1,000 tons $U_{3} 0_{8}$ annually (San Juan Basin Regional Uranium Study 1980).

ANNUAL PRODUCTION: None to date ${ }^{(b)}$

CUMULATIVE PRODUCTION: N.A.

RESERVES: 25 milliton lb $\mathrm{U}_{3} \mathrm{O}_{8}{ }^{\text {(b) }}$ (Perkins 1979)

DEPTH: Expected depth 3,400 ft (Perkins 1979)

GRADE: $0.14 \% \mathrm{U}_{3} 0_{8}{ }^{(b)}$ (Perkins 1979)

AGE OF MINE: Construction stopped in 1981; not yet fully operational, full production was expected in $1982-1983$, but now on a "hold"
position. (b)

OTHER COMMENTS: The mine has an expected 20 year 1 ife; the expected mining recovery efficiency is $95 \%$ of the ore in place (Perkins 1979).

(a) Information from T. G. Brough, Ph.D., Environmental Scientist, Radiation Protection Bureau, Environmental Improvement Division, Milan, New Mexico.

(b) Infomation from C. Wentz, Uranium Specialist, Energy and Minerals Department, Santa Fe, New Mexico. 
MINE NAME: Mariano Lake

COMPANY: Gulf Mineral Resources

LOCATION: T15N, R14W Section 12, MCKinley County, New Mexico

OPERATING STATUS: - Closed April, 1982 (Gulf 0il Corp. 1982)

- Still closed as of $2 / 83$, reported that mine is,flooded, shaft being filled and surface being restored. (a)

DESIGNED MINE CAPACITY: 625 tons ore/day (Perkins 1979)

ANNUAL PRODUCTION: N.A.

CUMULATIVE PRODUCTION: $\quad \geq 750,000$ tons (Perkins 1979)

RESERVES: - Now depleted (Gulf 0il Corp. 1982)

- Reserves before mining at 3.5 million $16 \mathrm{U}_{3} 0_{8}$ (Perkins 1979)

DEPTH: $510 \mathrm{ft}$ (Energy and Minerals Dept 1982)

GRADE: $\quad 0.24 \% \quad U_{3} 0_{8}$ with $0.10 \% \quad U_{3} 0_{8}$ cutoff (Perkins 1979).

AGE OF MINE: Began production 1977, closed (depleted) April, 1982 (Gulf 0il Corp. 1982).

OTHER COMMENTS: The mine had one vertical shaft, and one exhaust fan. With an air discharge of 83,000 (ACFM) (Perkins 1979). The mine area is presently under reclamation.

(a) Information from T. G. Brough, Ph.D., Environmental Scientist, Radiation Protection Bureau, Environmental Improvement Division, Milan, New lexico. 
MINE NAME: Velvet

COMPANY: Atlas Corporation, Minerals Division

LOCATION: T31S, R25E, SW 1/4 Section 3, San Juan County, Utah

OPERATING STATUS: Active as of $11 / 82$ (U.S. Department of Labor, 1982)

DESIGNED MINE CAPACITY: N.A.

ANNUAL PRDDUCTION: N.A.

CUMULATIVE PRODUCTION: N.A.

RESERVES: As of $6 / 30 / 82$, Atlas' interests in proven ore-bearing properties approximates $2,172,000$ tons reserves (At las Corp. 1982)

DEPTH: N.A.

GRADE: Ranges from $0.08-0.52 \%$ of economically recoverable $U_{3} 0_{8}$ (Atlas Corp. 1982)

AGE OF MINE: N.A.

OTHER COMMENTS: 
MINE NAME: Tony M (Lucky Strike)

COMPANY: Plateau Resources, Ltd.

LOCATION: T35S, R11E, Section 16 and 21, Garfield County, Utah

OPERATING STATUS: Active as of $11 / 82$ (U.S. Departiment of Labor, 1982)

DESIGNED MINE CAPACITY: N.A.

ANNUAL PRODUCTION: 1980-actual ore tonnage of 34,159 tons (E\&MJ, 1981)

CUMULATIVE PRODUCTION: N.A.

RESERVES: 10.9 million $10 \mathrm{U}_{3} \mathrm{O}_{8}$ under development (average grade $0.15+\%$ ), 6.3 million $16 \mathrm{U}_{3} \mathrm{O}_{8}$ (average grade $0.15+\%$ ) undeveloped (represents all Plateau Resources properties) (Consumers Power Co., 1982)

DEPTH: N.A.

GRADE: $\quad 0.05-0.15 \% U_{3} 0_{8}$ - represents a number of smal1 deposits in SE Utah held by Plateau Resources (Steyn, 1981)

AGE OF MINE: N.A.

OTHER COMMENTS: Plateau owns or has under lease or option 35,442 acres in Utah. These acres are mostly unpatented and nonproducing claims, with a small part being state leased and fee lands. Property holdings were reduced in 1981, (from 77,500 in 1980) to concentrate on activities in the Henry Mountains, Garfield, Co., Utah (Tony M Mine Area). The NRC issued Plateau a license on $9 / 21 / 79$ for a uranium processing facility near Shootaring Canyon. This unit will process ore mined from Plateau's properties. Work was suspended as of $3 / 7 / 81$ because of disputes with a construction company, Mountain States Mineral Enterprises, Inc. Construction was completed in the second quarter, 1982, under Plateau's supervision. Operations commenced June, 1982 and produced 27,267 lo of uranium concentrates in 1982. A decision was made to defer deliveries to the parent (Consumers Power) until 1985, resulting in temporary suspension of facility operation in August, 1982, along with a significant reduction in mining and processing staff levels. The anended contract with Consumers Power called for delivery of $9,100,000$ lbs contained $\mathrm{U}_{3} \mathrm{O}_{8}$ in concentrates over a 13 year period. Consumers Power has a net investment in Plateau of $\$ 73.9$ million as of $12 / 31 / 82$ (Consumer Power Co., 1982). 
MINE NAME: Wilson-Silverbell

COMPANY: Union Carbide Corporation

LOCATION: T32S, R26E, SW 1/4, Section 15, San Juan County, Utah

OPERATING STATUS: ACtive as of $11 / 82$ (U.S. Department of Labor, 1982)

OESIGNEO MINE CAPACITY: N.A.

ANNUAL PRODUCTION: N.A.

CUMULATIVE PRODUCTION: N.A.

RESERVES: N.A.

DEPTH: N.A.

GRADE: N.A.

AGE OF MINE: N.A.

OTHER COMMENTS: 
MINE NAME: Lisbon

COMPANY: Rio Algom Corporation

LOCATION: T29S, R24E, SE 1/4, Section 21, San Juan Country, Utah

OPERATING STATUS: Active as of 11/82 (U.S. Department of Labor, 1982)

DESIGNED MINE CAPACITY: (Integrated mine and mill) Mill capacity increased from 500 to 700 tons per day (Rio Algom, Ltd., 1982).

ANNUAL PRODUCTION: 1982 - 123,000 tons of Lisbon ore processed, or 454,000 ib of $\mathrm{U}_{3} \mathrm{O}_{8}$ in concentrate which was stockpiled. During 1982 , mine production was at $50 \%$ normal rate as implemented in October, 1981 (Rio Algom, Ltd., 1982).

CUMULATIVE PRODUCTION: N.A.

RESERVES: Aggregated at 388,000 tons ore as of $12 / 82$. This is noted as proven and probable ore with $31 \mathrm{~b} \mathrm{U} \mathrm{U}_{3}$ per ton ore cutoff. These aggregated reserves equal 1.2 million $\mathrm{lb} \mathrm{V}_{3} \mathrm{O}_{2}$, recoverable after allowing for milling losses (Rio Algom, Ltd., 1982).

OEPTH: 2,650 ft final depth (Rio Algom, Ltd., 1982)

GRAOE: $1.8 \% \mathrm{U}_{3} \mathrm{O}_{8}$ average recovered grade for 1982 . This equals a mine grade of 3.37 ib of $\mathrm{U}_{3} \mathrm{O}_{8}$ per ton of ore after allowing for losses in mine extraction and difution (Rio Algom, Ltd., 1982).

AGE OF MINE: Production began 10/1/72 (Rio Algom, Ltd., 1982)

OTHER COMMENTS: The Lisbon Mine has two shafts and is mined by room and pillar operation. Production work force was reduced $50 \%$ on $10 / 81$

(Rio Algom, Ltd., 1982). 


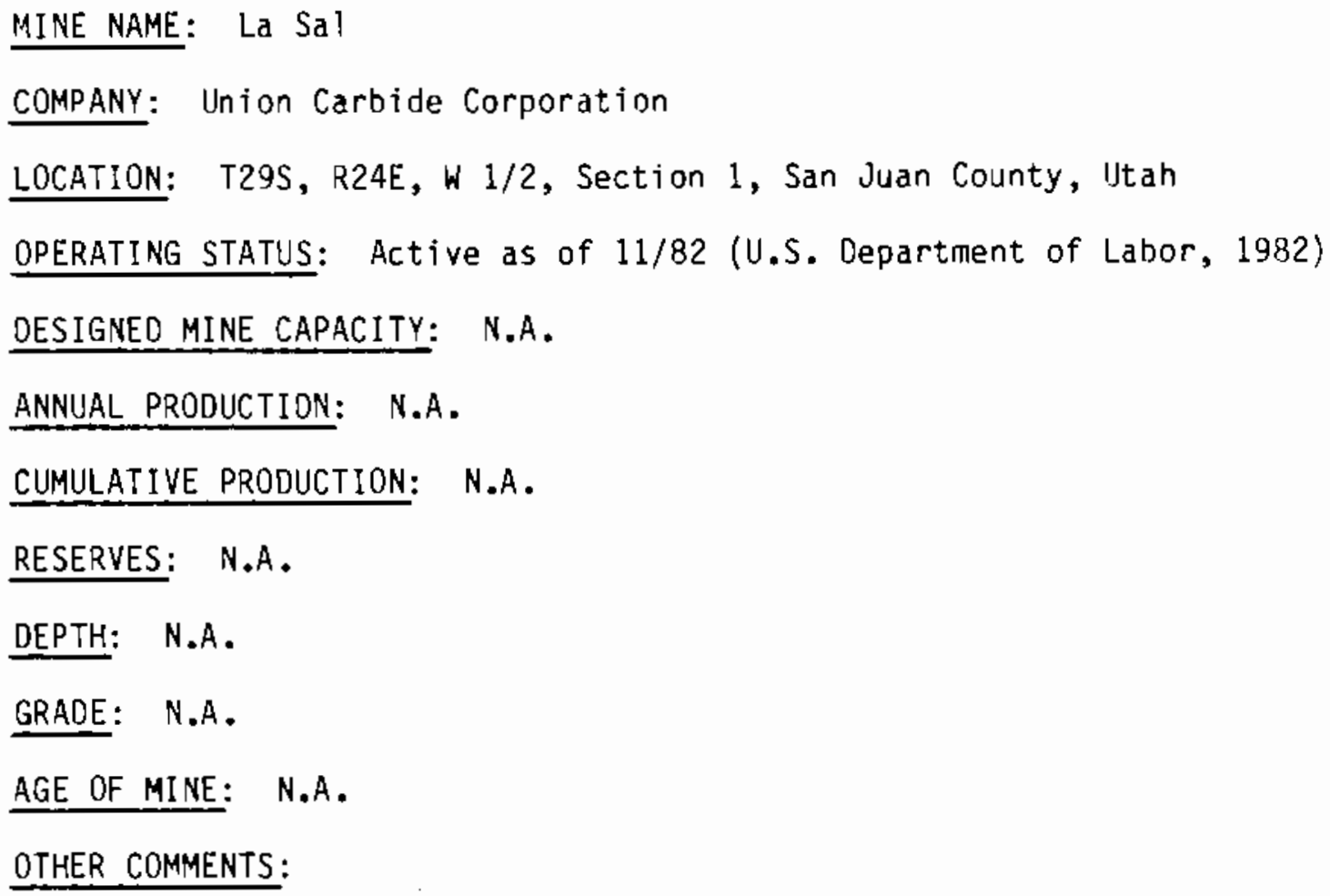


MINE NAME: Hecla

COMPANY: Union Carbide Corporation

LOCATION: T29S, R24E, N 1/2, Section 6, San Juan County, Utah

OPERATING STATUS: Active as of 11/82 (U.S. Departinent of Labor, 1982)

DESIGNED MINE CAPACITY: N.A.

ANNUAL PRODUCTION: N.A.

CUMULATIVE PRODUCTION: N.A.

RESERVES: N.A.

DEPTH: N.A.

GRADE: N.A.

AGE OF MINE: N.A.

OTHER COMMENTS: 
MINE NAME: Golden Eagle

COMPANY: Silver King Mines, Inc.

LOCATION: T36N, R73W, Sec. 14 Converse County, Wyoming

OPERATING STATUS: under development $(\mathrm{a})$

DESIGNATED MINE CAPACITY: N.A.

ANNUAL PRODUCTION: No production yet, still being developed as of $6 / 83$. (a)

CUMULATIVE PROOUCTION: N.A.

RESERVES: N.A.

DEPTH: N.A.

GRADE: Average ore $\left(\mathrm{g}\right.$ )ade is $0.15 \% \mathrm{U}_{3} \mathrm{O}_{8}$ as reported in thesis work by
Ray Harris.

AGE OF MINE: N.A.

OTHER COMMENTS: - It will be 3-5 years befoceproduction begins, depending on the market conditions. (a)

- Silver King Mines has a management contract with the Tenessee Valley Authority (TVA) for the supervision of exploration, development and production on uranium/vanadium properties in Wyoming (Golden Eagle).

On May 11, 1978, the Company expanded the initial contract with the Tennessee Valley Authority for supervision of continued exploration, mine development, mining and milling operations, and construction of mine surface facilities and a uranium ore mill. This contract, which was for two years and has been extended for two years with a 10 year option to renew, stipulates that Silver king is to receive a base monthly fee of $\$ 19,417$ plus reimbursement for additional authorized expenditures and increases computed in accordance with fluctuations in the consumer price index from the date of the agreement.

The aforementioned base monthly fee has exceeded $\$ 25,000$ since March 31, 1980. Further, the Tenessee Valley Authority has ayreed to pay additional monthly fees to Silver King currently agyregating $\$ 12,000$ (Silver King Mines 1982).

(a) Information from Ray Harris, Soft Rock Minerals Geologist, The Geological Survey of Wyoming, Laramie, Wyoming. 
MINE NAME: Big Eagle

\section{COMPANY: Pathfinder Mines Corporation}

LOCATION: T27N, R92W, Sec. 2 \& 11 Fremont County, Wyoming

OPERATING STATUS: active as of $6 / 83^{(a)}$

DESIGNATED MINE CAPACITY: Production capacity stated as 400,000 tons ore per year by 1979 (Goodier 1978).

ANNUAL PRODUCTION: 1982152,122 tons ore $(\mathrm{a})$

1981260,686 tons ore

1980455,997 tons ore

1979118,513 tons ore

1978104,472 tons ore

CUMULATIVE PRODUCTION: $1978-82: \quad 1,091,790$ tons ore as of $12 / 31 / 82^{(a)}$

RESERVES: in 1978,7 million lb $\mathrm{U}_{3} \mathrm{O}_{8}$ proven (Goodier 1978).

DEPTH: N.A.

GRADE: $0.15 \% \quad U_{3} 0_{8}$ (1978) (Steyn 1981)

AGE OF MINE: began operation in $1978^{(a)}$

OTHER CDMMENTS:

(a) Information from Ray Harris, Soft Rock Minerals Geologist, The Geological Survey of Wyoming, Laramie, Wyoming. 
MINE NAME: Sheep Mountain Operations

COMPANY: Western Nuclear, Inc.

LOCATION: T28N, R92W Sec. 22, Fremont County, Wyoming

OPERATING STATUS: - Inactive as of $6 / 83(a)$

- The mine has been shut down and on standby maintenance since mid-1981 (Phelps Dodge Corp. 1982).

DESIGNATED MINE CAPACITY: N. A.

ANNUAL PRODUCTION: $1983--0(\mathrm{a})$

$1982-0$

1981--119,147 tons ore

CUMULATIVE PROOUCTION: 1955-1983: $4,519,518$ tons ore produced from the Sheep Mountain complex, Crooks Gap District. (a) The Sheep Mountain complex, Crooks Gap District, is made up of the following mines:

Sheep Mountain \#1

McIntosh

Golden Goose

Seismic

Reserve

Congo

RESERVES: as of $1978,7.2$ million pounds U308 proven. Indicated reserves to last 20 years (Goodier 1978 ).

DEPTH: N.A.

GRADE: - a weighted (ayyerage grade for the production/period 1955-1973 is

- 1978 average grade reported as $0.12 \%$ U308 (Steyn 1981).

AGE OF MINE: Sheep Mountain operations began in 1955 (a)

OTHER COMMENTS: Hestern Nuclears' mines are now on standby status but could be back in operation on very short notice if the demand for uranium oxide so warranted (Phelps Dodge Corp. 1982).

(a) Information from Ray Harris, Soft Rock Minerals Geologist, The Geological Survey of Wyoming, Laramie, Wyoming. 
MINE NAME: Schwartzwalder

COMPANY: Cotter Corporation, a wholly owned subsidiary of Commonwealth Edison of Chicago.

LOCATION: T2S, R71W, Sec. 25, Jefferson County, Colorado

OPERATING STATUS: ACtive as of $11 / 82$ (U.S. Dept. of Labor 1982)

DESIGNATED MINE CAPACITY: - daily ore capacity of 500 tons ore per day (Wright 1980)

- 600 tons ore per day (E \& MJ 1981).

ANNUAL PRODUCTION: 1980: 100,000 to 500,000 short tons ore (E \& MJ 1981). CUMULATIVE PRODUCTION: N.A.

\section{RESERVES: N.A.}

DEPTH: The mine is much deeper than it is broad in any aerial dimension. It is $2000 \mathrm{ft}$. deeper than it was when Allen Birds did a thesis study on the mine in 1958 (Wright 1980). GRADE: $\begin{aligned} 0.30 \% U_{3} 0_{8}(E \& M J 1981) \cdot 0.20-0.25 \% U_{3} 0_{8} \text { with a } 0.09 \% \text { cutoff } \\ (\text { Wright } 1980)\end{aligned}$

AGE OF MINE: opened in 1956 (E \& MJ 1981).

OTHER COMMENTS: This mine is the premier vein-type uranium deposit. It has three shafts and is mined by open stopping of the vein. By products of mining are molybdenum and silver $(0.1-2.002$ silver/ton ore) (E\&MJ 1981).

The Illinois vein is up to $50 \mathrm{ft}$. wide, with narrower horsetails averaging 1.5 feet wide. All uranium produced by the mine is committed to use by Commonwealth Edison (Wright 1980). 
MINE NAME: Bill Smith

COMPANY: Kerr-MCGee Corporation

LOCATION: T36N, R74W Sec. 36, Converse County, Wyoming

OPERATING STATUS: Underground mine closed. Environmental requirements have stopped all but one open pit operation (Steyn 1981).

DESIGNATED MINE CAPACITY: N.A.

ANNUAL PRODUCTION: $1979-83 \quad 0^{(a)}$

19785,482 tons ore

19779,098 tons ore

CUMULATIVE PROOUCTION: 1977-1978: 14,580 tons ore. (a)

RESERVES: 60 million lbs. (?) $\mathrm{U}_{3} \mathrm{O}_{8}$ reserves in-place estimated (Steyn 1981)

DEPTH: N.A.

GRADE: N.A.

AGE OF MINE: began operation in $1977^{(a)}$

OTHER COMMENTS: The underground mine went on standby status in 1978, with no production since ${ }^{(a)}$ (Goodier 1978).

(a) Information from Ray Harris, Soft Rock Minerals Geologist, The Geological Survey of Wyoming, Laramie, Wyoming. 
."

.

$\checkmark$

.

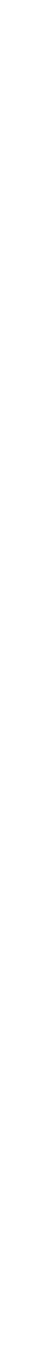


APPENDIX D

STUDY MINE LOCATION AND DATA MAPS 


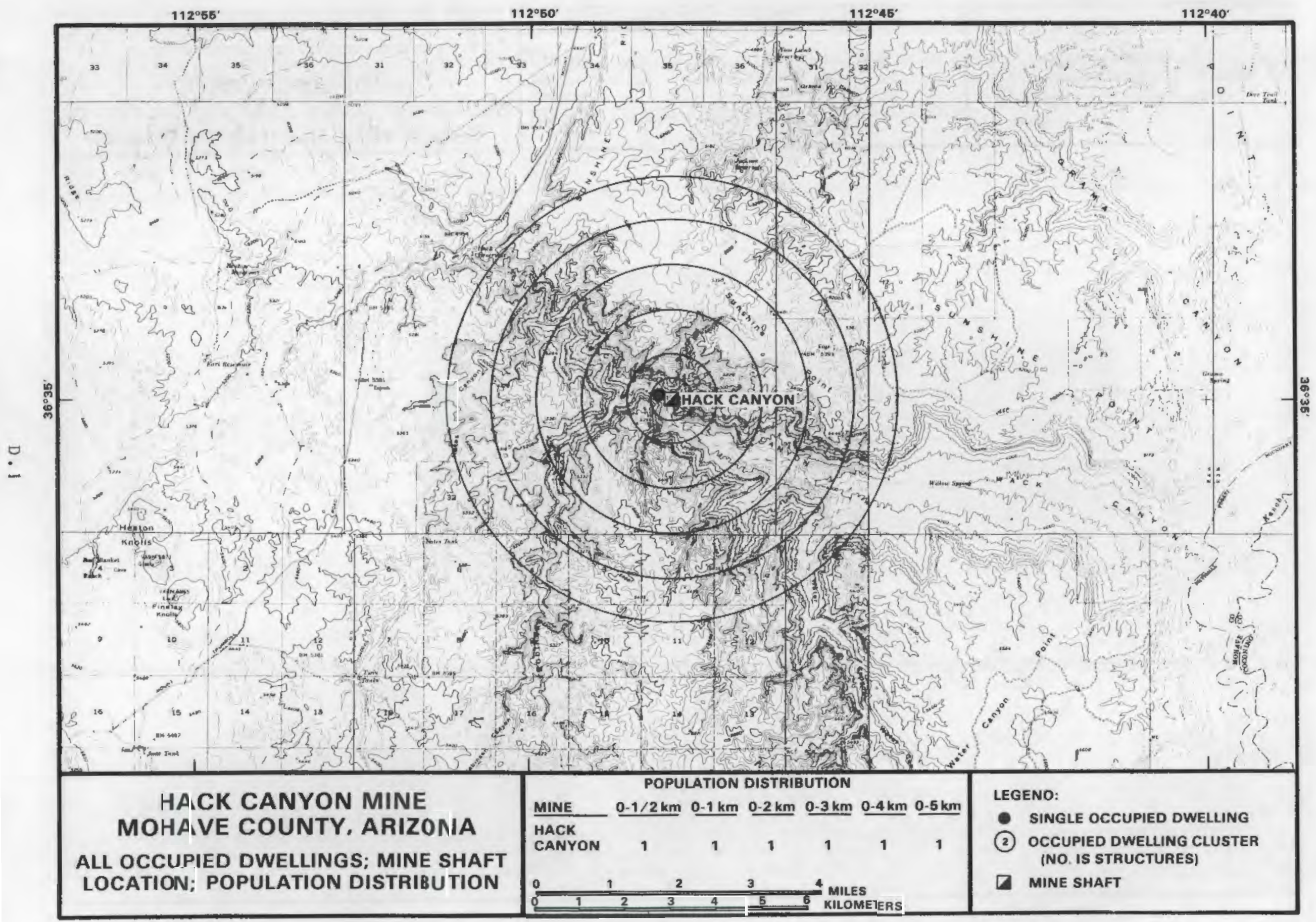




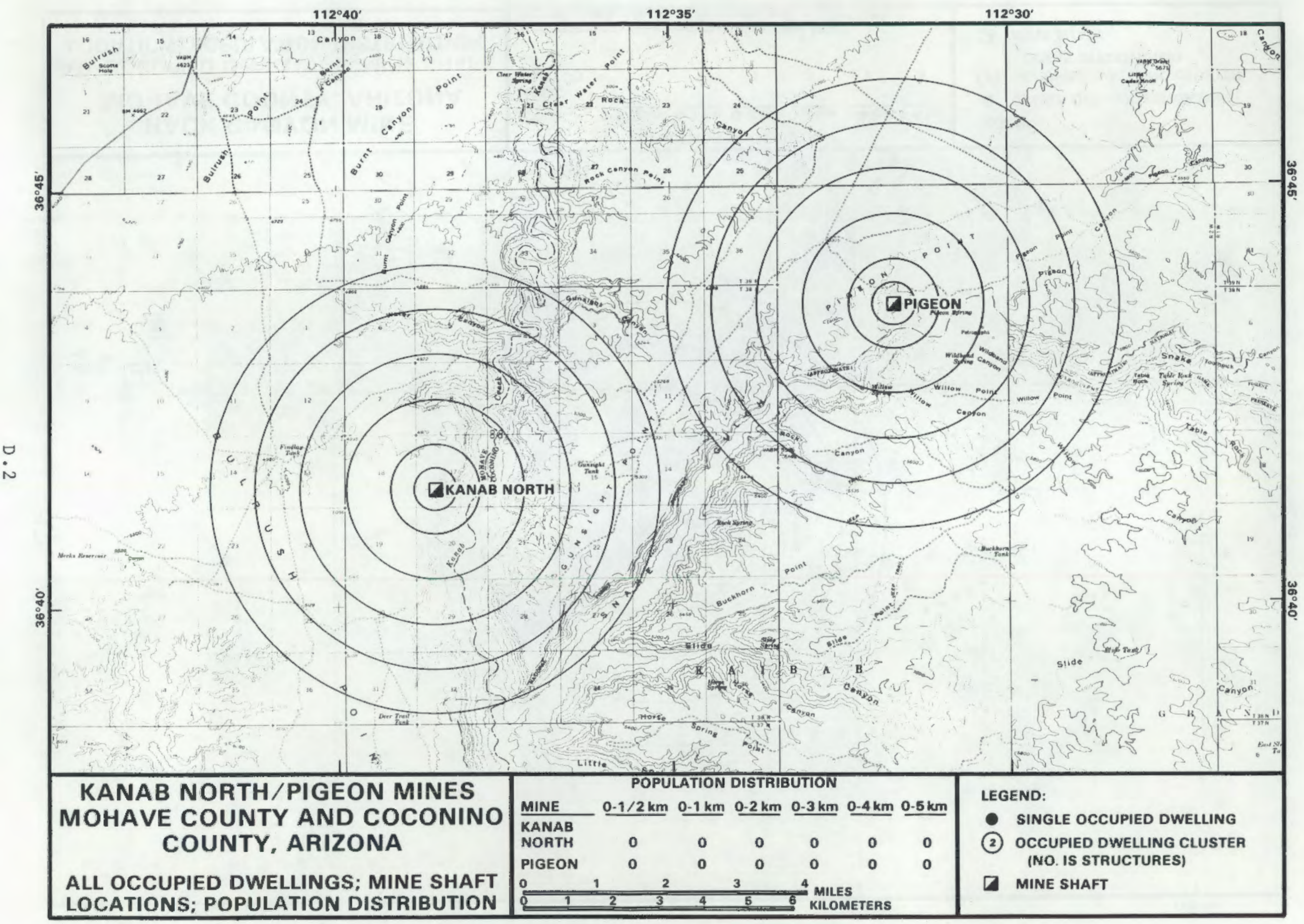




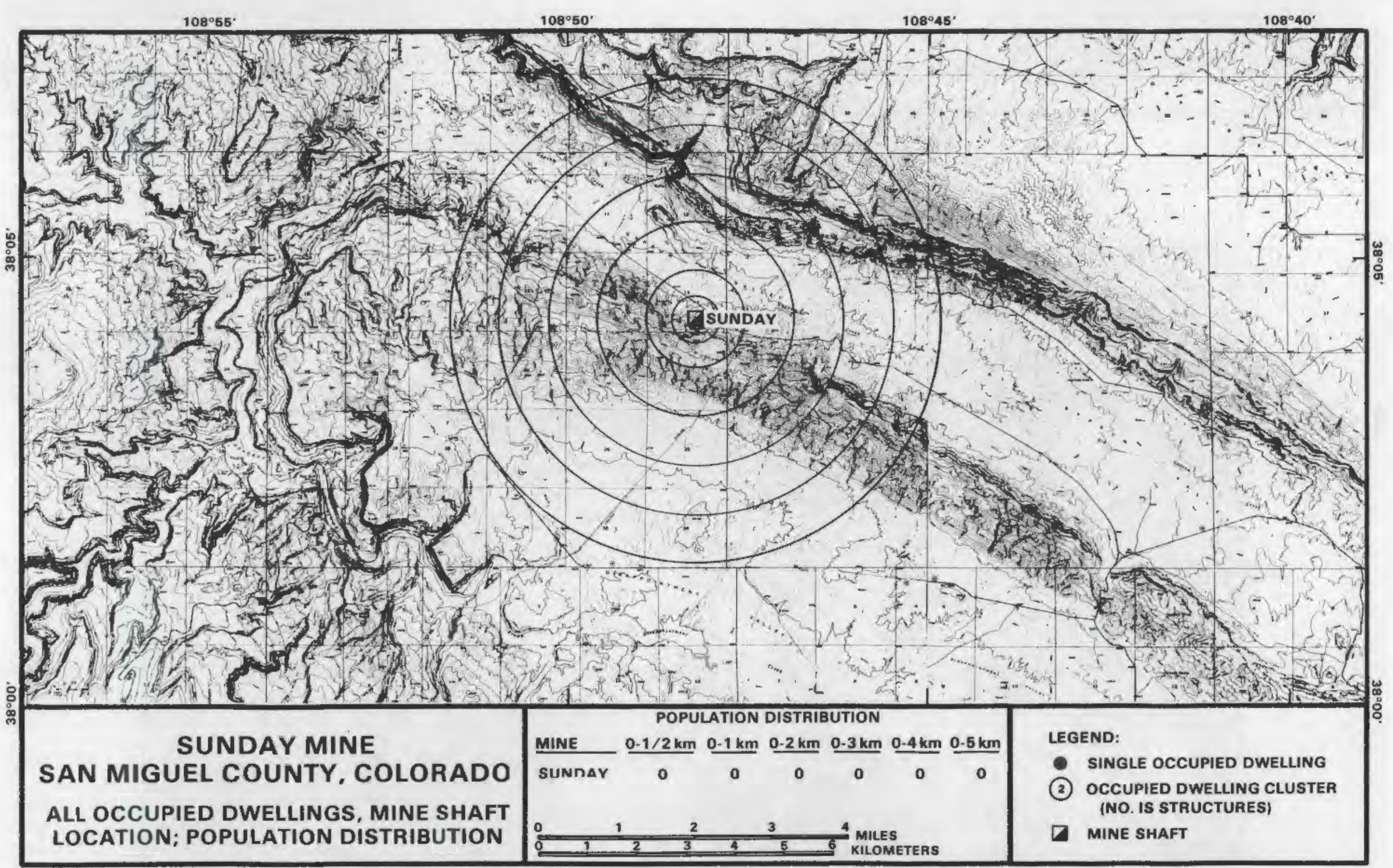




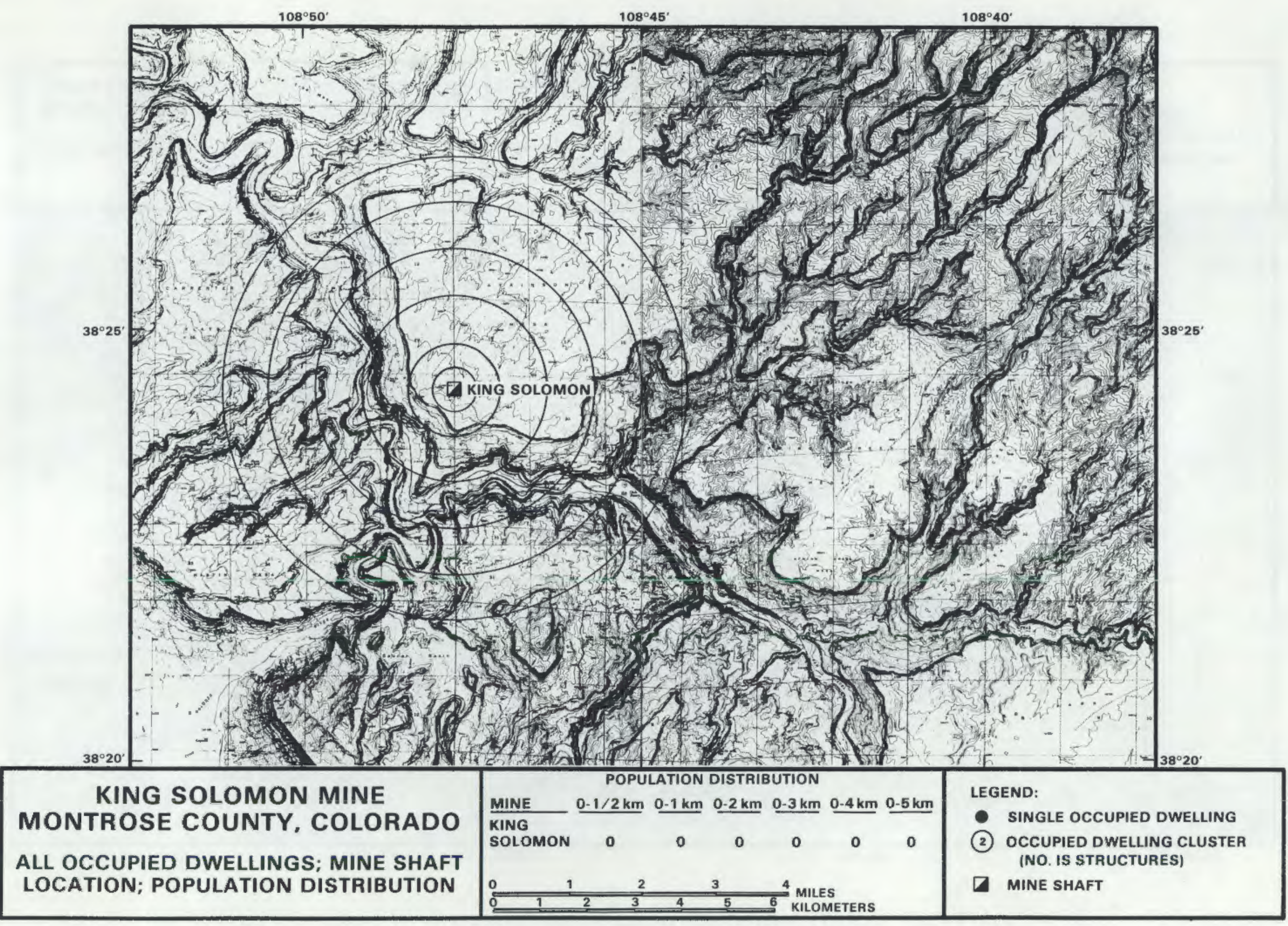




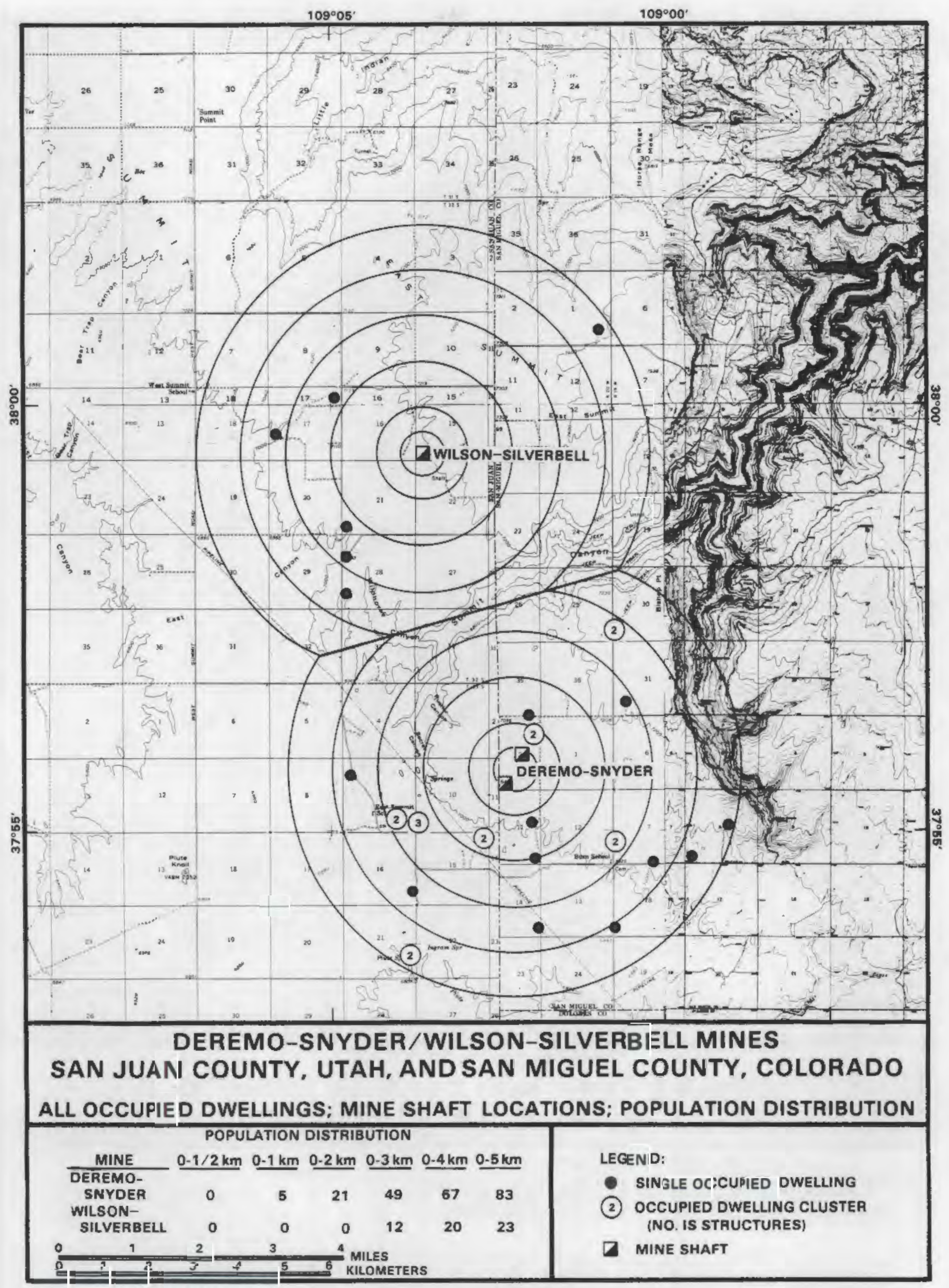




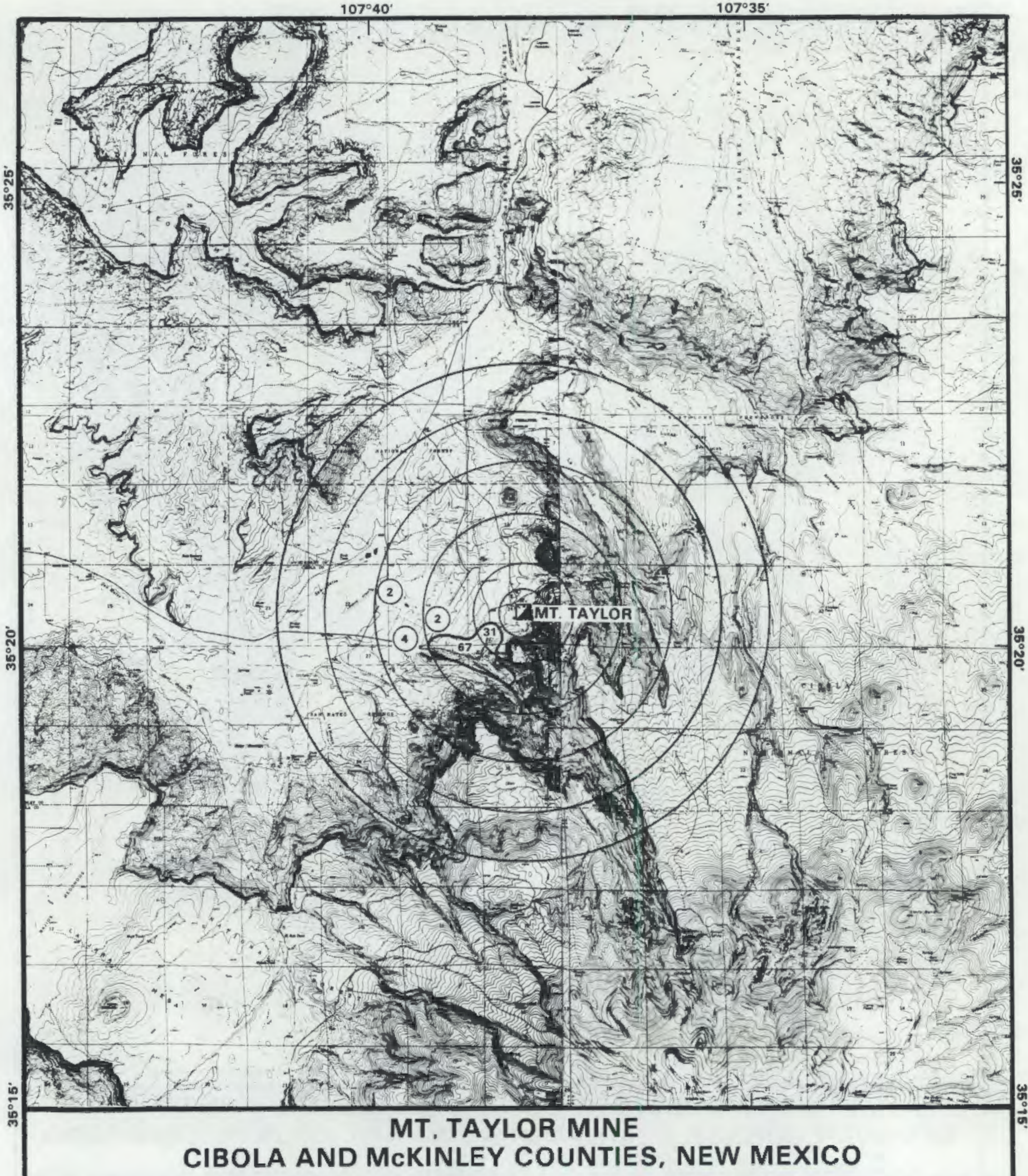

ALL OCCUPIED DWELLINGS; MINE SHAFT LOCATION; POPULATION DISTRIBUTION POPULATION DISTRIBUTION

$\frac{\text { MINE }}{\text { MT.TAYLOR }} \frac{0.1 / 2 \mathrm{~km}}{0} \frac{0-1 \mathrm{~km}}{100} \frac{0.2 \mathrm{~km}}{317} \frac{0-3 \mathrm{~km}}{336} \frac{0.4 \mathrm{~km}}{336} \frac{0.5 \mathrm{~km}}{336}$

LEGEND:

- single occupied dWelling

(2) OCCUPIED DWELLING CLUSTER (NO. IS STRUCTURES)

0

12

$\frac{3}{4}$ 


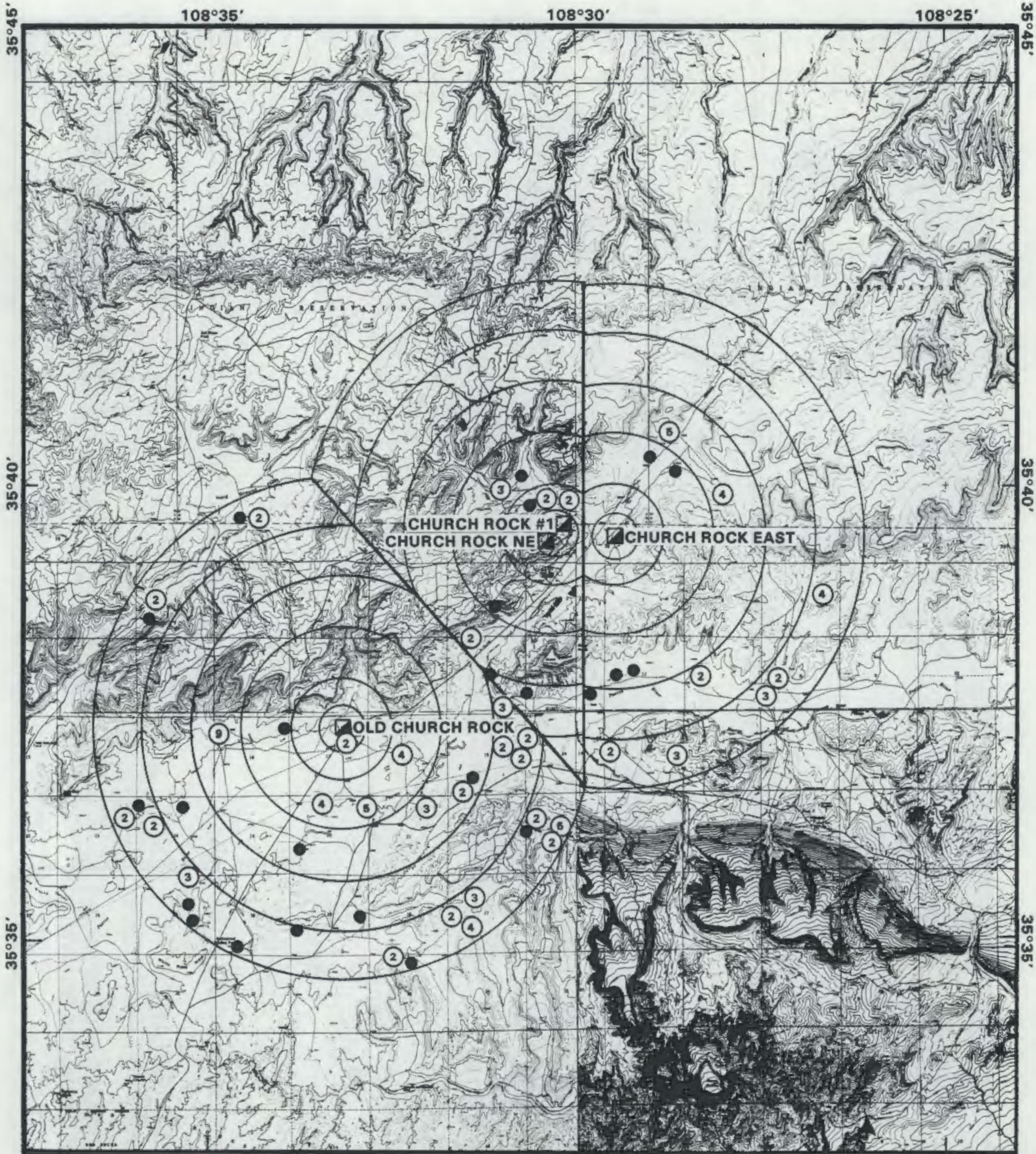

\section{CHURCH ROCK MINES, McKINLEY COUNTY, NEW MEXICO}

\section{ALL OCCUPIED DWELLINGS; MINE SHAFT LOCATIONS; POPULATION DISTRIBUTION}

POPULATION DISTRIBUTION

MINE $0-1 / 2 \mathrm{~km} 0-1 \mathrm{~km} \quad 0.2 \mathrm{~km} \quad 0.3 \mathrm{~km} \quad 0.4 \mathrm{~km} 0.5 \mathrm{~km}$

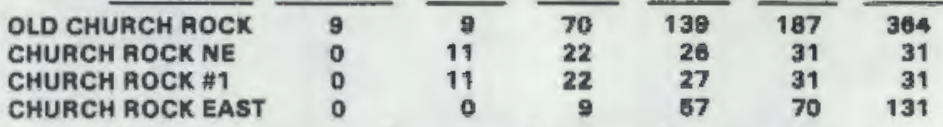

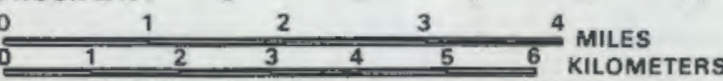

LEGEND:

- Single occupied DWELLING

(2) OCCUPIED DWELLING CLUSTER (NO. IS STRUCTURES)

MINE SHAFT 


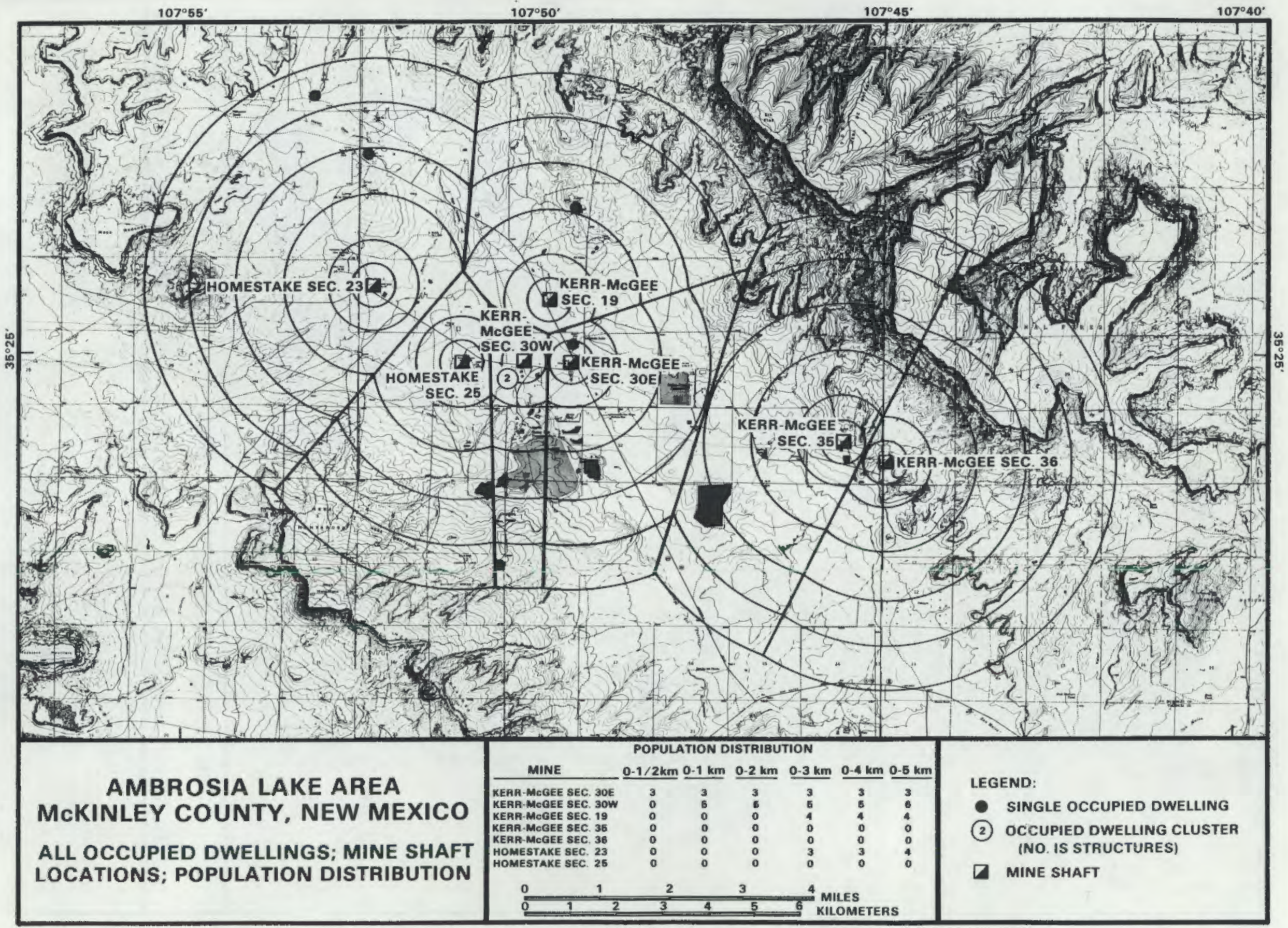




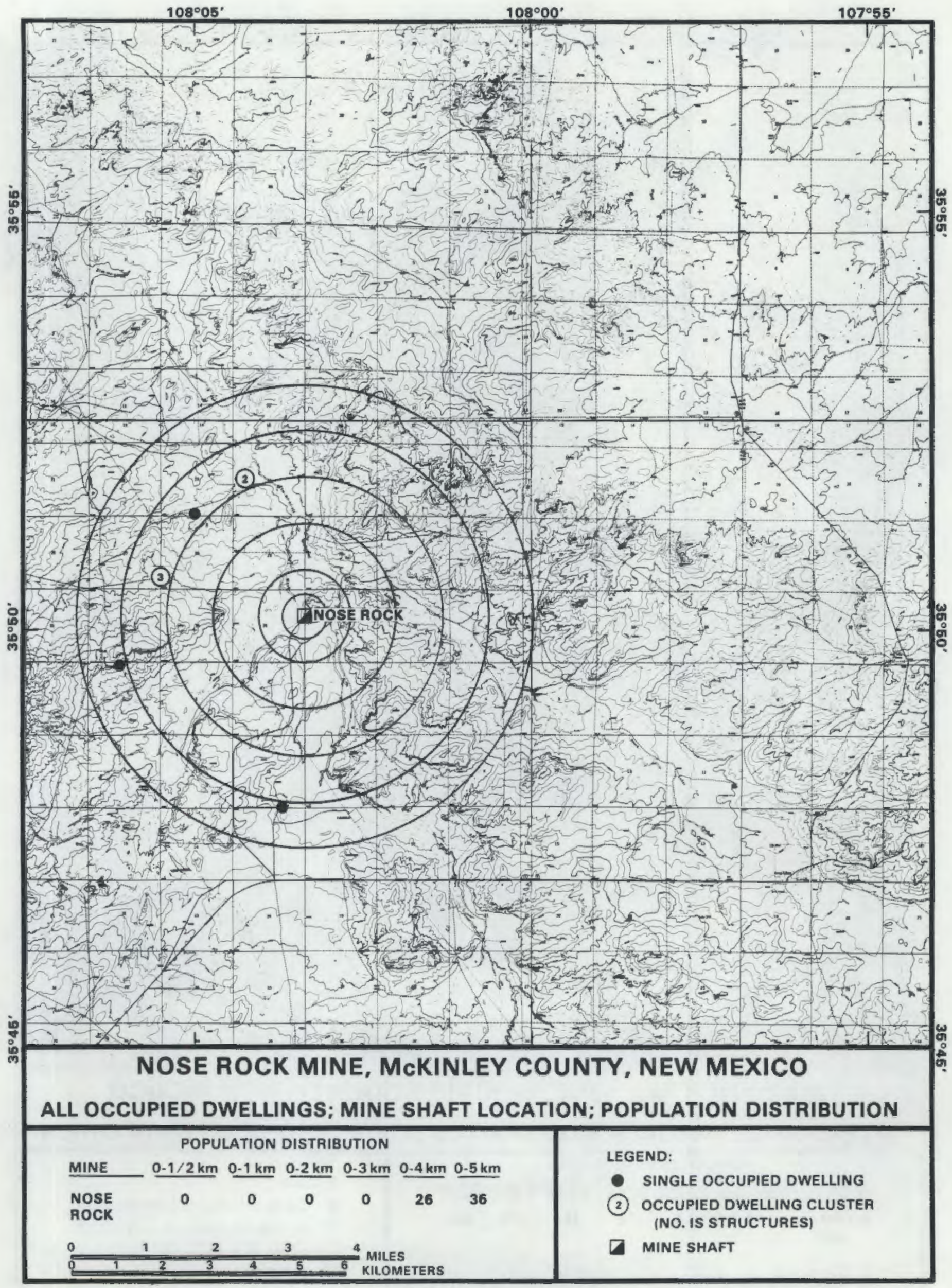




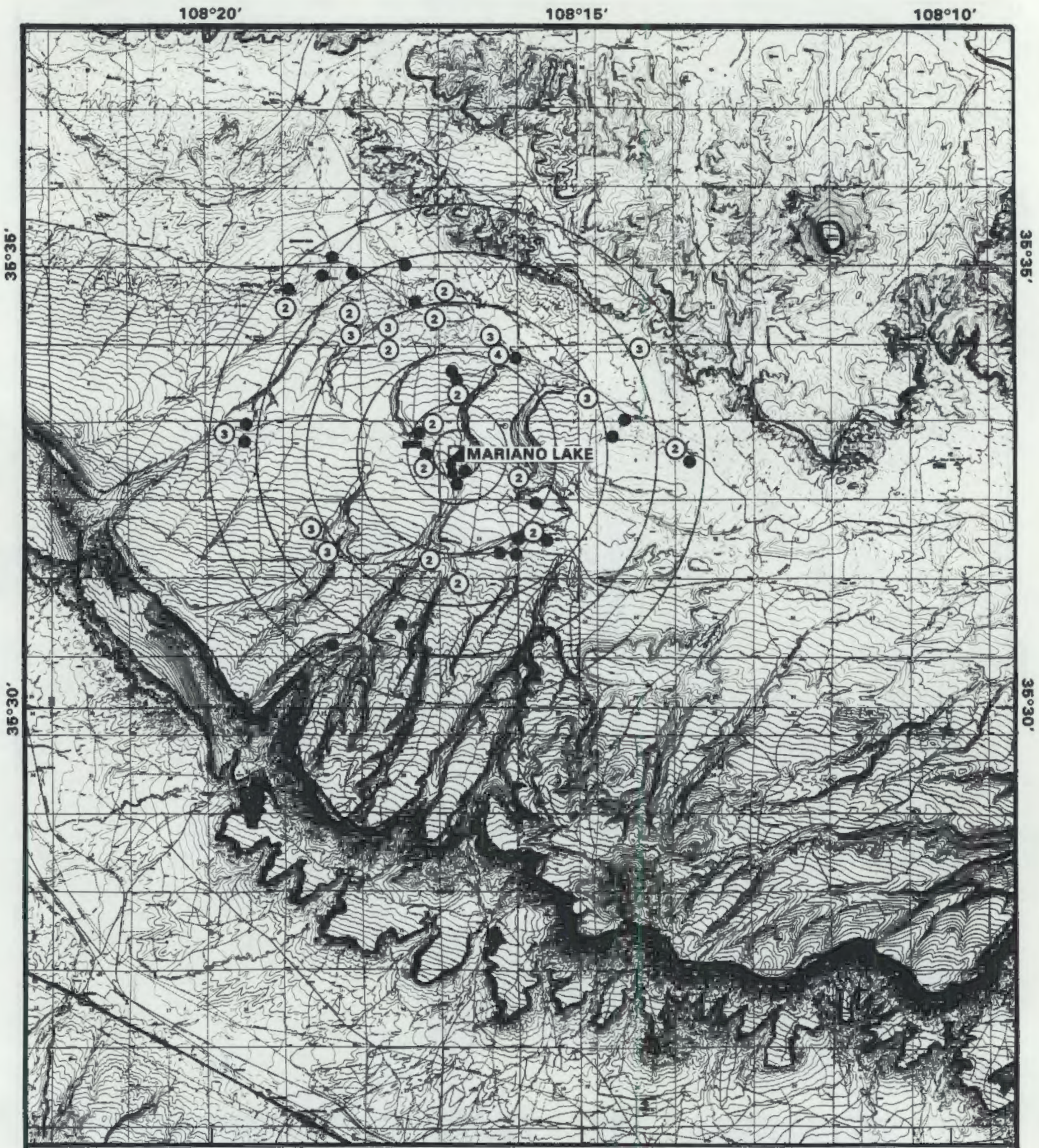

MARIANO LAKE MINE, MCKINLEY COUNTY, NEW MEXICO

ALL OCCUPIED DWELLINGS; MINE SHAFT LOCATION; POPULATION DISTRIBUTION

POPULATION DISTRIBUTION

MINE $0.1 / 2 \mathrm{~km} 0.1 \mathrm{~km} 0.2 \mathrm{~km} 0.3 \mathrm{~km} 0.4 \mathrm{~km} 0.5 \mathrm{~km}$

$\begin{array}{lllllll}\text { MARIANO } & 13 & 44 & 76 & 196 & 274 & 352\end{array}$

LAKE

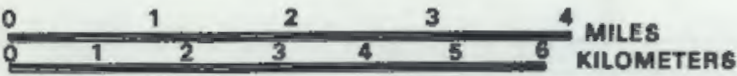

LEGEND:

- SINGLE OCCUPIED DWELLING

(2) OCCUPIED DWELLING CLUSTER (NO. IS STRUCTURES)

MINE SHAFT 


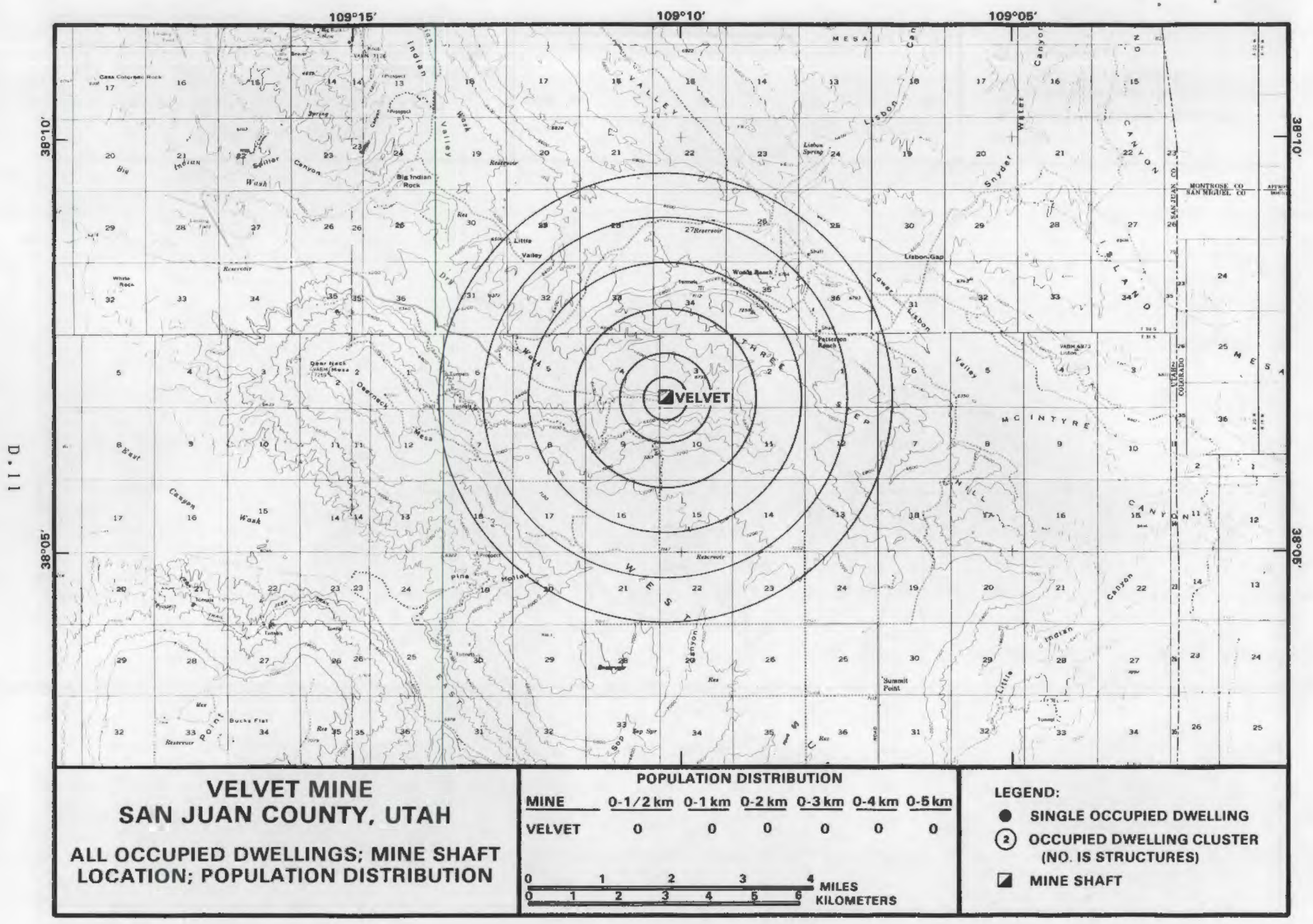




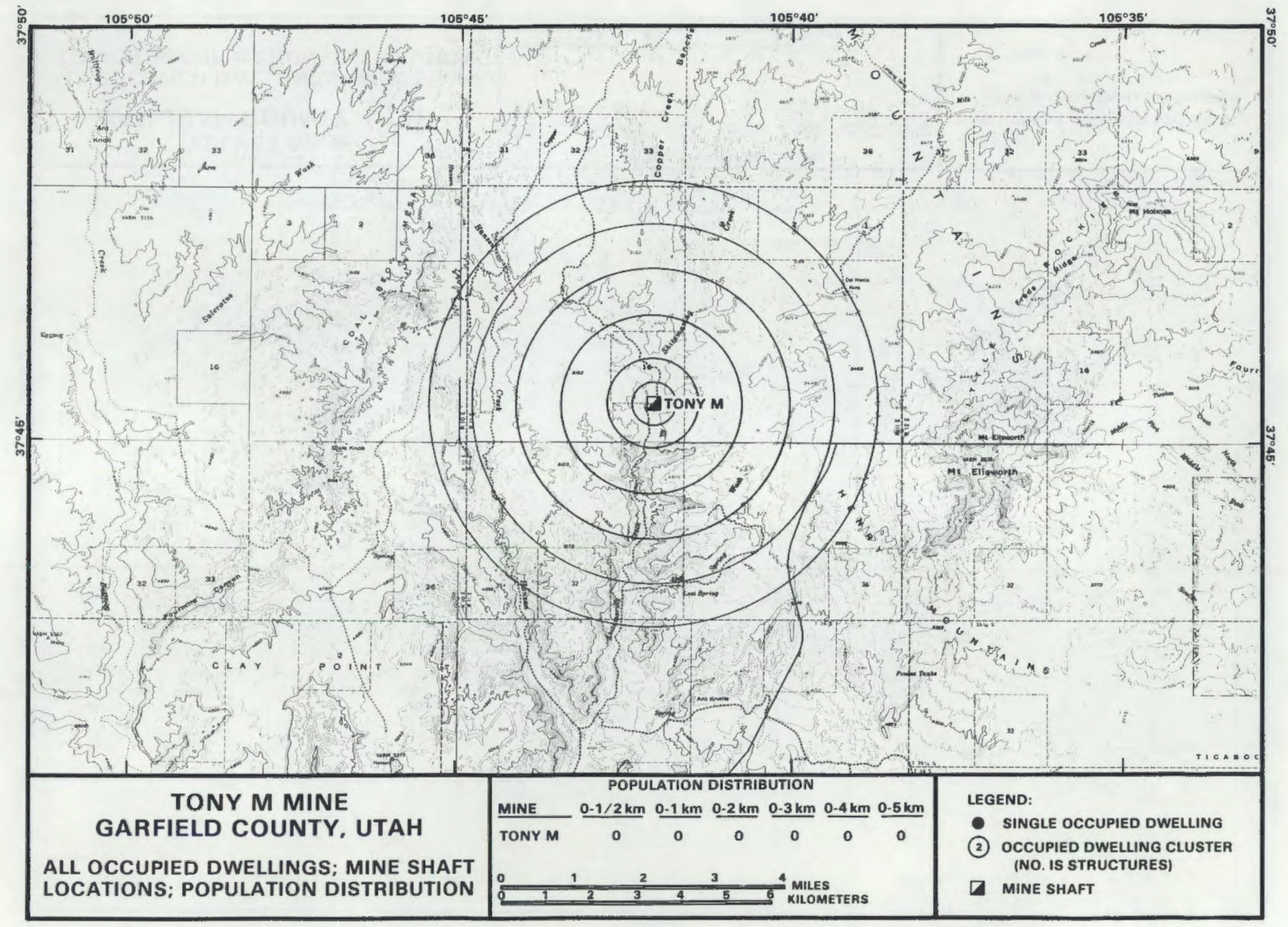




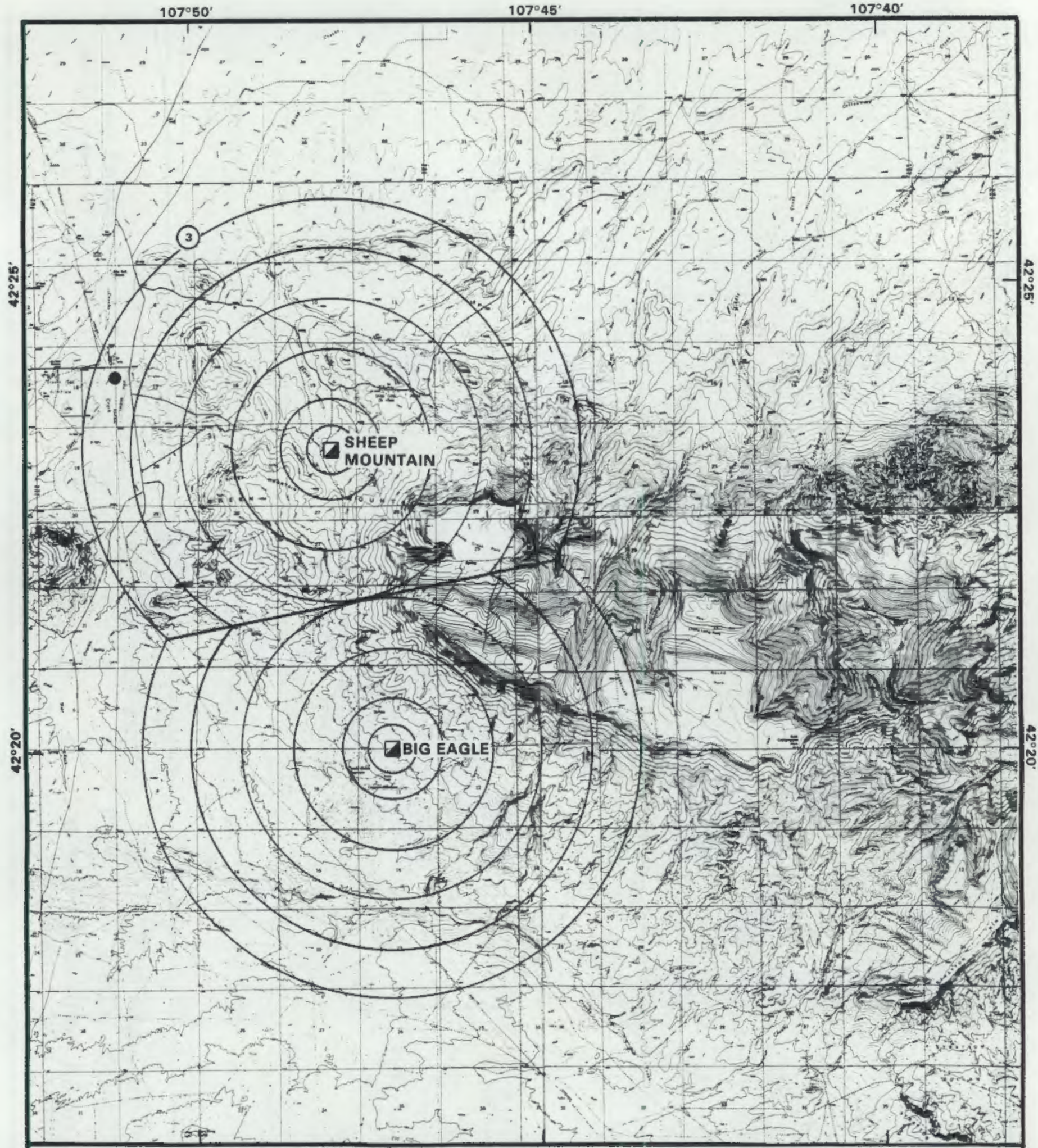

BIG EAGLE/SHEEP MOUNTAIN MINES, FREMONT COUNTY, WYOMING

ALL OCCUPIED DWELLINGS; MINE SHAFT LOCATIONS; POPULATION DISTRIBUTION POPULATION DISTRIBUTION

$\frac{\text { MINE }}{\text { BIG EAGLE }} \frac{0.1 / 2 \mathrm{~km}}{0} \frac{0.1 \mathrm{~km}}{0} \frac{0.2 \mathrm{~km}}{0} \frac{0.3 \mathrm{~km}}{0} \frac{0.4 \mathrm{~km}}{0} \frac{0.5 \mathrm{~km}}{0}$

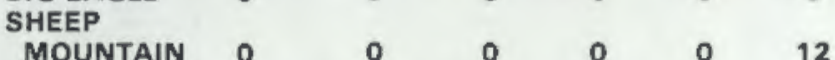

MOUNTAIN $0 \quad 0 \quad 0000012$

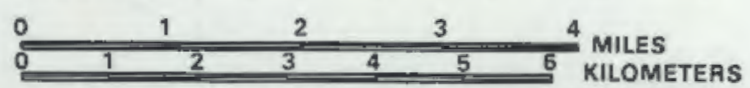

LEGEND:

- SINGLE OCCUPIED DWELLING

(2) OCCUPIED DWELING CLUSTER (NO. IS STRUCTURES)

MINE SHAFT 


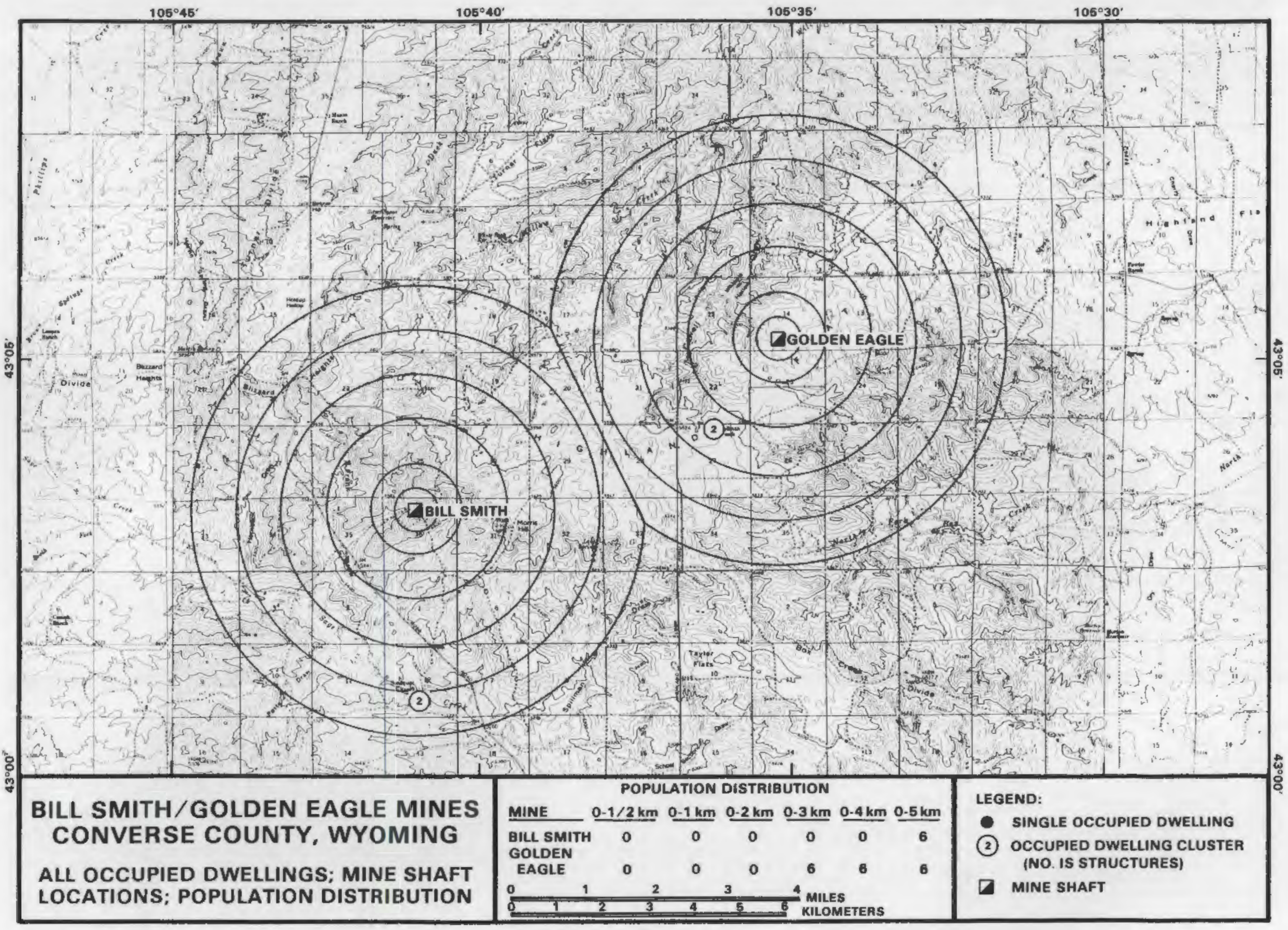




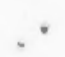

$-$ 
APPENDIX E

ASSOCIATED MINE FACILITIES DESCRIPTIONS 
ARIZONA

Hack Canyon Mine

Energy Fuels Nuclear, Inc.

Mohave County, Arizona

The primary uses for the land in the vicinity are for mining, mineral exploration, stock range, and unconfined recreation. Vegetation consists of low shrubs, range grasses, and scattered groves of juniper. Cattle were evident on the mesa in the vicinity of Hack Canyon. There were also numerous claim markers in the area and mineral exploration activity was also evident. The mine property is bounded on three sides (West, South and East) by proposed Wilderness Study Areas (WSA's). These areas are designated as 1-34 and 1-33A as shown on the WSA's reference map for Arizona. These areas are also discussed in the Wilderness Review Arizona report (U.S. Dept. of Interior, BLM, Arizona State Office, 1980).

Pigeon Mine Energy Fuels Nuclear, Inc. Coconino County, Arizona

The primary uses for the land in the vicinity of the mine site are mineral exploration, stock range, and unconfined recreation. Logging operations are also conducted in the Kaibab National Forest, to the south of the property. Vegetation in the vicinity of the mine site consists of low shrubs, range grasses and groves of juniper. Cattle were evident in the vicinity of the mine site. Numerous claim markers were also evident in the area.

The nearest boundary of any proposed Wilderness Study Area (WSA) is about two miles $(3.2 \mathrm{~km})$ to the west, designated as $1-31$ on the WSA's reference map for Arizona. This area is also discussed in the Wilderness Review Arizona report (U.S. Dept. of Interior, BLM, Arizona State Office, 1980).

Kanab North

Energy Fuels Nuclear, Inc.

i" Mohave County, Arizona

The primary uses for the land in the vicinity of the proposed mine site are mineral exploration, stock range, and unconfined recreation. Vegetation 
consists of low shrubs and range grasses. The mine site is located within the proposed WSA unit 1-31. This unit is identified on the WSA reference map for Arizona. This study area is also discussed in the Wilderness Review Arizona report (U.S. Dept. of Interior, BLM, Arizona State Office, 1980). There is currently considerable opposition to this mining project by the environmental community (The National Wildlife Federation and the South West Regional Council). 
COLORADO

Sunday Mine

Union Carbide Corporation

San Miguel County, Colorado

The Sunday Mine is located in an area of NW-SE trending ridges and valleys in southwestern Colorado. The Big Gypsum and Disappointment Valleys traverse the study area with the Sunday Mine site situated in an intervening mountain ridge. The primary uses of the land are for mining, mineral exploration, stock grazing and unconfined recreation. Valleys contain mainly grasses and shrubs while mountain areas are tree covered.

The Hamm Canyon, a major feature after which the USGS quadrangle is named, drains southward into the Big Gypsum Valley. There is a pipeline traversing the study area north-south, passing within one mile $(1.6 \mathrm{~km})$ of the mine site. An airstrip is located in Section 35, T44N, R18W. Several other mining prospects occur in the northeast and eastern parts of the study area.

Three proposed Wilderness Study Areas (WSA's) surround the Sunday Mine study boundary. The Tabeguache Creek, Sewemup Mesa and Dolores River Canyon have their nearest boundaries at 12,6 and $8 \mathrm{miles}(19.2,9.6$, and $12.8 \mathrm{~km})$ respectively, and are designated as 030-300, 030-310A/070-176 and 030-290 on the Colorado BLM Intensive Wilderness Inventories-Final Wilderness Study Areas map. These areas are discussed in the Intensive wilderness Inventory report issued in November, 1980 (U.S. Dept. of Interior, BLM, Colorado State Office, 1980).

King Solomon Mine Union Carbide Corporation Montrose County, Colorado

The King Solomon Mine is tocated in a rugged mountain area of southwest Colorado. The west flowing San Miguel River meets the south flowing Dolores River within the study area and then exits to the south. The river drainages are deeply incised with valley walls rising up to 1000 feet high. Martin Mesa forms the southwestern portion of the area, Carpenter Flats the northwestern, while Atkinson Mesa lies central to northeast within the area. The primary 
uses of the land are for mining, mining exploration, stock grazing and unconfined recreation. The area is mostly tree covered with grasses and shrubs growing on open mesas and valley floors.

Numerous mining prospects line the rims of the rivers with some old placer operations occurring along the main rivers. There is no private property within a five krm radius of the mine while $97 \%$ of the land is publicly administrated.

The Dolores River Canyon proposed Wilderness Study Area (WSA) lies 8 miles $(12.8 \mathrm{~km})$ north of the $\mathrm{king}$ Solomon study boundary. It is designated as 030290 on the Colorado BLM Intensive Wilderness Inventories-Final Wilderness Study Areas map. This area is discussed in the Intensive Wilderness Inventory report issued in November, 1980 (U.S. Dept. of Interior, BLM, Colorado State Office, 1980). 


\section{NEW MEXICO}

Mt. Taylor Mine

Gulf Mineral Resources

Cibola County, New Mexico

The primary uses of the land in the vicinity of the Mt. Taylor Mine are for mining, mineral exploration, stock grazing, residential living and unconfined recreation. The site is bounded to the north, west and south by private residential property and ranching land. The village of San Mateo, population about 311 , is within $1 / 4 \mathrm{mile}(0.4 \mathrm{~km})$ to the southwest, lying within the four sections of $23,24,25$, and 26 . These residential properties are generally long, narrow parcels most of which border the San Mateo Creek which flows to the northwest through the area. The surrounding ranch land is largely owned by the Fernandez Company, Ltd., (9 to 10 sections) and the Michael Ranch, Inc. (less than $1 / 20$ section).

The Lee Ranch dwelling complex is situated about $13 / 4$ miles $(2.8 \mathrm{~km})$ to the west. The vegetation type in these areas is low shrubs and range grasses. Trees are scarce.

The Cibola National Forest boundary is $1 / 4 \mathrm{mile}(0.4 \mathrm{~km})$ east of the mine site. The flora here is mainly conifer trees, increasing in density with higher elevations. The San Lucas Dam and Reservoir are located about three miles $(4.8 \mathrm{~km})$ to the north, having a private access road. A main power distribution line runs $\mathrm{NW}-\mathrm{SE}$ and is $21 / 2$ miles $(4 \mathrm{~km})$ distant to the north.

There are no wilderness study areas within the greater mine vicinity in the Albuquerque BLM district as indicated in the New Mexico Wilderness Study Area Decisions report (U.S. Dept. of Interior, BLM, New Mexico State Office, 1980).

Church Rock \#1 and Church Rock East

Kerr-McGee Corporation

Mckinley County, New Mexico

01d Church Rock and Church Rock NE

UNC Resources, Inc.

McKinley County, New Mexico

In the Church Rock District, these four mines are clustered together and are located 10-15 miles (16-24 km) northeast of the city of Gallup, New Mexico. 
The greatest distance between any mine in the group is about four miles (6.4 $\mathrm{km}$ ) (01d Church Rock and Church Rock East). The primary uses of the land in the area are for mining, mineral exploration, and Indian usage for perhaps subsistence and stock grazing. A recreation site is available at the Red Rock State Park near the village of Church Rock $(10 \mathrm{l} / 2 \mathrm{~km})$ south of the $01 \mathrm{~d}$ Church Rock mine site. Any other unconfined recreation is probably limited due to Indian control of lands.

There are only three parcels of privately owned land within the encompassing five $\mathrm{km}$ radius of the mine cluster. The largest property is only about one section in size, with the other two being $1 / 4$ sections each. The Navajo Indian Reservation border 1 ies $1 / 2 \mathrm{mile}(0.8 \mathrm{~km})$ north of the Church Rock $\# 1$, Church Rock NE, and Church Rock East mines. Other than about $11 / 4$ section of company owned property, all remaining lands are Navajo Indian allotted.

The vegetation type is mainly low shrubs and range grasses. Conifer trees occur above the 7000 foot elevation. One outstanding physiographic feature is the Ram Mesa in section 11.

The UNC uranium processing plant which is situated near the Churck Rock \#1 and Churck Rock NE mines in section 2 and has associated tailings ponds. A major power transmission line runs east-west and passes one mile $(1.6 \mathrm{~km})$ south of the 01d Churck Rock mine.

There are no wilderness study areas within the greater mine vicinity of the Albuquerque BLM District as indicated in the New Mexico Wilderness Study Area Decisions report (U.S. Dept. of Interior BLM, New Mexico State Office, $1980)$.

Kerr-McGee Sections 19, 30E, 30w, 35 and 36 Mines

Kerr-McGee Corporation

Mckinley County, New Mexico

Homestakes Sections 23 and 25 Mines

Homestake Mining Company

Mckinley County, New Mexico

In the Ambrosia Lake District, these 7 mines are clustered together, centered roughly on the old Ambrosia townsite. The greatest distance between any mines in the cluster is 7 miles $(11.2 \mathrm{~km}$ ) (distance between Homestake section 
23 and Kerr McGee section 36). The primary uses of the land in the vicinity of the Ambrosia Lake mine cluster are for mining, mineral exploration, stock grazing and minor unconfined recreation. In regards to mining, the Ambrosia Lake environs is literally peppered with both active and inactive mining operations. Many old hoist head frames dot the landscape along with mining equipment of al1 types. Security guards in the hire of the still active operations patrol the grounds. Little if any exploratory activity is presently underway.

Within the encompassing $5 \mathrm{~km}$ radii of the mines in the cluster, 8 major private property owners (owning greater than one section each) hold lands used mainly for ranching. These include the Harris, Roundy, Berryhill Enterprises, Marquez, Fernandez Co., Ltd and other properties. There are about 8 minor (owning less than one acre) private property owners within the same area. In addition, several sections are designated as Navajo Indian allotted Iands. The vegetation on this ranching and Indian land is low shrubs and range grasses.

There are two uranium mills within the 7-mine cluster. These are the active Kermac Nuclear Fuels Processing Plant (Sec. 31, T14N, R9W) and the inactive Phillips Petroleum Ambrosia Mill (Sec. $28 \mathrm{~T} 14 \mathrm{~N}$, R9W). Each facility has associated mill tailings ponds of different sizes--about one section total area for Kermac, and 1/4 section for Phillips. There is a power transmission line serving the Kermac Plant. This line runs approximately east-west and lies about $1 / 2 \mathrm{mile}(0.8 \mathrm{~km})$ north of and parallel to the T13 and $14 \mathrm{~N}$ dividing 1 ine.

The Cibola National Forest boundary is $11 / 2 \mathrm{miles}(2.4 \mathrm{~km})$ north and $41 / 2$ miles $(7.2 \mathrm{~km}$ ) south from the nearest mine(s) (Kerr McGee Section 35 and 36).

Conifer trees are present on the slopes an tops of the San Mateo Mesa and the La Jara Mesa (to the south) within the Cibola National Forest.

There are no wilderness study areas in the greater mine vicinity in the Albuquerque BLM District as indicated in the New Mexico Wilderness Study Area Decisions report (U.S. Dept. of Interior, BLM, New Mexico State Office, 1980). Nose Rock Mine Phillips Petroleum Company Mckinley County, New Mexico

The Nose Rock Mine is located in open, sparsely populated country about $13 \mathrm{miles}(20.8 \mathrm{~km})$ north of Crownpoint, New Mexico. The primary uses for the E.7 
land are for mining, mineral exploration and Indian usage for perhaps subsistence and stock grazing.

The Chaco Canyon National Park (an Indian Ruins) is the closest recreational site located roughly 20 miles $(32 \mathrm{~km}$ ) to the northeast. Any other unconfined recreation in the immediate mine area is probably limited due to Indian property.

Phillips owns or has surface rights to $35 \%$ of the area within $5 \mathrm{~km}$ of its mine site. Of the 13 sections encompassed in full or part by this $5 \mathrm{~km}$ radius, 11 sections have been acquired by Phillips as part of a land exchange with the Navajo Indian Tribe. The other 2 sections are Phillips property acquired by other means.

The only physiographic feature is the Nose Rock land formation just north of the $5 \mathrm{~km}$ radius. Two north flowing drainages run through the area, the Kim-me-ni-oli and Seven Lakes Washes. Several small stock watering reservoirs lie peripheral to the study area. And finally, a power transmission line runs NW-SE about $1 \mathrm{mile}(1.6 \mathrm{~km})$ west of the mine site with a substation located in Sec. 18, T18N, R11W.

There are no wilderness study areas within the greater mine vicinity in the Albuquerque District, as indicated in the New Mexico Wilderness Study Area Decisions report (U.S. Dept. of Interior, BLM, New Mexico State Office, 1980).

Mariano Lake

Gulf Mineral Resources

McKinley County, New Mexico

The Mariano Lake is located on $100 \%$ indian and pubijcly owned land. In the vicinity of the mine, the major land uses are for mining, mineral exploration, and Indian usage. Recreational uses are probably minimal. The continental Divide is $43 / 4 \mathrm{mi}(7.6 \mathrm{~km})$ to the east, while to the north 1.9 miles $(3 \mathrm{~km}$ ) is a Bureau Mission and northwest $3 \mathrm{miles}(4.8 \mathrm{~km})$ is Mariano Lake and the Mariano Lake Village. Hosta Butte, a major physiographic landmark, lies $53 / 4$ miles $(9.2 \mathrm{~km})$ to the northeast. A power distribution line passes 1 mile $(0.8 \mathrm{~km})$ to the north of the mine area, traversing E-W through the district. 
The primary vegetation type is low shrubs, range grasses, and sparse conifer trees in the valleys with increasing tree density at higher elevations.

There are no wilderness study areas within the greater mine vicinity in the Albuquerque BLM District, as indicated in the New Mexico Wilderness Study Area Decisions report (U.S. Dept. of Interior, BLM, New Mexico State Office, 1980). 
UTAH

Velvet Mine

Atlas Corporation

San Juan County, Utah

The primary uses of the land in the vicinity of the Velvet Mine are for mining, mineral exploration, stock grazing and unconfined recreation. The area lies midway between the Lisbon and Wilson-Silverbell study areas, in a high mountain park called the West Sumit. The vegetation type is range grasses, shrubs, and conifer stands.

The Lisbon Valley and Lower Lisbon Valley border the study area to the north and northeast while the Three Step Hill range forms the central part of the area. Mining prospects occur in the north and west portion of the area. The Colorado border lies four miles $(16.4 \mathrm{~km})$ to the east.

There are no wilderness study areas (WSA's) in the greater vicinity on the Utah side of the Utah/Colorado border. Approximately 12 miles (19.2 km) to the east is the boundary of the Dolores River Canyon WSA in Colorado. This is designated as 030-290 on the Colorado BLM Intensive Wilderness InventoriesFinal Wilderness Areas map. This area is discussed in the Intensive Wilderness Inventory report issued in November, 1980 (U.S. Dept. of Interior, BLM, Colorado State office, 1980).

Tony M (Lucky Strike) Mine

PI ateau Resources, Ltd.

Garfieid County, Utah

The primary uses for the land in the vicinity of the Tony M Mine are for mining, mineral exploration, stock range land, and unconfined recreation. The Starr Ranch is located about six miles $(9.6 \mathrm{~km})$ northeast of the mine property. The Starr Springs campground is also located in the same area. There are no campsites closer than this to the mine property. Vegetation in the vicinity is mainly low shrubs and range grasses.

The nearest boundaries of the Mt. Pennell, Mt. Ellsworth, and Mt. Hillers proposed Wilderness Study Areas (WSA's) are approximately six miles (9.5 km) from the mine property. These areas are designated as 050-247, 050-248, and 
050-249 on the Wilderness Inventory reference map for the State of Utah. These areas are discussed in the Utah Intensive Wilderness Inventory report (U.S. Dept. of Interior, BLM, Utah State Office, 1980).

Wilson-Silverbell/Deremo-Snyder Union Carbide Corporation

San Juan County, Utah and San Miguel County, Colorado

This mining area is located on the Utah/Colorado border in a high mountainous area called the East Summit. The elevation is about 7000 feet and above. The primary uses of the land in the vicinity are for mining, mineral exploration, stock grazing, and unconfined recreation. The vegetation type is range grasses, low shrubs, and conifer stands. The Summit Cannon lies midway between the two sites, draining the countryside to the northeast.

A pipeline passes about one mile, southwest of the Deremo-Snyder site, running in a NW-SE direction. Overal1, $81 \%$ of the land is privately owned with only $9 \%$ being in public administration.

There are no wilderness study areas/(WSA's) in the greater vicinity on the Utah side of the Utah/Colorado border. Approximately 12 miles $(19.2 \mathrm{~km})$ to the northeast is the boundary of the Dolores River Canyon WSA in Colorado. This is designated as 030-290 on the Colorado BLM Intensive Wilderness InventoriesFinal wilderness Study Areas map. This area is discussed in the intensive Wilderness Inventory report issued in November, 1980 (U.S. Dept. of Interior, BLM, Colorado State Office, 1980).

Lisbon/LaSal/Hecla Mines

Rio Algom Limited/Union Carbide Corporation/Union Carbide Corporation San Juan County, Utah

The primary uses of the land in the vicinity of these three adjoining mine areas are for mining, mineral exploration, stock grazing, and unconfined recreation. The vegetation type varies from range grasses and sagebrush to stands of conifer trees. The Manti-LaSal National Forest borders the Hecla/LaSal areas to the north and east. The Mt. Tukuhnikivatz, Mt. Peale, Mt. Mellenthin trio lie within this forest about $6-8 \mathrm{miles}(9.6-12.8 \mathrm{~km})$ north of the study areas. 
The West Coyote Creek drains the areas to the west, passing through the LaSal Junction site at about $11 / 2$ miles $(2.4 \mathrm{~km})$ west of the Hecla area. The town of LaSal lies in Section 2, T29S, R24E, within the LaSal mine area.

The Lisbon and Big Indian Valleys trend NW-SE, entering the three site areas from the south. The Colorado border lies 6 miles $(9.6 \mathrm{~km})$ to the east of the LaSal area. Overall, 74-83\% of the land is publicly administrated.

The Mill Creek and Behind the Rock Wilderness Study Areas (WSA's) each have their boundaries about $10 \mathrm{miles}(16 \mathrm{~km})$ to the northwest of the study areas' boundary. These are designated as 060-139 and 060-140 on the wilderness Inventory reference map for the State of Utah. These areas are discussed in the Utah Intensive Wilderness Inventory report, (U.S. Dept. of Interior, BLM, Utah State office, 1980). 
WYOMING

Sheep Mountain Mine and Big Eagle Mine Western Nuclear. Inc., and Pathfinder Mines Corporation Fremont County, Wyoming

These two adjoining areas are located in sparsely populated country about $71 / 2$ miles $(12 \mathrm{~km})$ and $12 \mathrm{miles}(19.2 \mathrm{~km})$ respectively southeast of Jeffrey City. The primary uses of the land are for mining, mineral exploration, ranching, and unconfined recreation. The vegetation type is mainly range grasses and sagebrush. Pockets of more dense sagebrush, grasses, aspen, and juniper trees occur with higher elevations and along the drainages.

In describing the Sheep Mountain mine area, there is the Sheep Creek which flows northerly and passes by the site. Sheep Mountain, which is part of the larger Green Mountain Range, rises to the west and south of the site, both forming the major physiographic landforms of the region. Numerous mining prospects dot the mountain slopes. A strip mined area lies 2 to $21 / 2$ miles $(3.2-4 \mathrm{~km})$ to the southwest. The Sheep Creek 0il Field is located $1 \mathrm{mile}$ $(1.5 \mathrm{~km})$ to the northeast.

In describing the Big Eagle mine area, there are the Fourth and Jost Creeks which feed the larger Crooks Creek. These all form the major drainage flowing east to west across the southern part of the study areas. Crooks Creek is surrounded by State land along most of its course through the area. There are four public water resource blocks of land totalling 5/16 section within the Big Eagle Area. Crooks Creek reservoir lies $3 / 4$ mile $(1.2 \mathrm{~km})$ to the southwest. Green mountain rises to the north.

The closest boundaries of any proposed Wilderness Study Areas (WSA's) are the Sweetwater Rocks' areas which lie about $15 \mathrm{miles}(24 \mathrm{~km})$ to the northeast. They are designated as 030-120 and 030-122 on the Wyoming wilderness Study map. These areas are discussed in the Wyoming Wilderness Study AreasFinal Inventory Report, (U.S. Dept. of Interior, BLM, Wyoming State Office, 1981). 
Bill Smith and Golden Eagle Mines

Kerr-McGee Corp. and Silver King Mines Company

Converse County, Wyoming

The primary uses of the land in the vicinity of these two adjoining areas are for mining, mineral exploration, ranching, and unconfined recreation. Vegetation is sparse, consisting of only low shrubs and range grasses with trees only around ranch dwellings areas or in wet drainages. Many small seasonal watering reservoirs dot the areas between the two mine sites. Most drainages and creeks are seasonal as well. There are no outstanding land features. Also, there are no wilderness study areas in this Platte River Resource areas of the Casper BLM District as indicated in the Wyoming Wilderness Study Areas - Final Inventory Report (U.S. Dept. of Interior, BLM, Wyoming State Office, 1981).

Three uranium mills surround the sites. The active Exxon mill is 6 miles $(19.6 \mathrm{~km})$ east of the Golden Eagle mine site. The inactive Spook mill site is about 8 miles $(12.8 \mathrm{~km})$ north while the active Bear Creek mill lies 10 miles $(16 \mathrm{~km})$ north. 


\section{APPENDIX $F$}

PRESIDENT REAGAN'S STATEMENT OF NUCLEAR ENERGY POLICY 
APPENDIX F

\section{PRESIDENT REAGAN'S STATEMENT OF NUCLEAR ENERGY POLICY}

A more abundant, affordable, and secure energy future for all Americans is a critical element of this Administration's economic recovery program. While homeowners and business firms have shown remarkable ingenuity and resourcefulness in meeting their energy needs at lower cost through conversation, it is evident that sustained economic growth over the decades ahead will require additional energy supplies. This is particularly true of electricity, which will supply an increasing share of our energy.

If we are to meet this need for new energy supplies, we must move rapidly to el iminate unnecessary government barriers to efficient utilization of our abundant, economical resources of coal and uranium. It is equally vital that the utilities--investor-owned, public, and co-ops--be able to develop new generating capacity that will permit them to supply their customers at the lowest cost, be it coal, nuclear, hydro, or new technologies such as fuel cells.

One of the best potential sources of new electrical energy supplies in the coming decades is nuclear power. The U.S. has developed a strong technological base in the production of electricity from nuclear energy. Unfortunately, the federal government has created a regulatory environment that is forcing many utilities to rule out nuclear power as a source of new generating capacity, even when their consumers may face unnecessarily high electric rates as a result. Nuclear power has become entangled in a morass of regulations that do not enhance safety but that do cause extensive licensing delays and economic uncertainty. Government has also failed in meeting its responsibility to work with industry to develop an acceptable system for commercial waste disposal, which has further hampered nuclear power development.

To correct present government deficiencies and to enable nuclear power to make its essential contribution to our future energy needs, I am announcing today a series of policy initiatives: 
1. I am directing the secretary of energy to give immediate priority attention to recommending improvements in the nuclear regulatory and licensing process. I anticipate that the chairman of the Nuclear Regulatory Commission will take steps to facilitate the licensing of plants under construction and those awaiting licenses. Consistent with public health and safety, we must remove unnecessary obstacles to deployment of the current generation of nuclear power reactors. The time involved to proceed from the planning stage to an operating license for new nuclear power plants has more than doubled since the mid-1970s and is presently some 10-14 years. This process must be streamlined, with the objective of shortening the time involved to $6 \rightarrow 8$ years, as is typical in some other countries.

2. I am directing that government agencies proceed with the demonstration of breeder reactor technology, including completion of the Clinch River Breeder Reactor. This is essential to ensure our preparedness for longer-term nuclear power needs.

3. I am lifting the indefinite ban which previous Administrations placed on commercial reprocessing activities in the United States. In addition, we will pursue consistent, long-term policies concerning reprocessing of spent fuel from nuclear power reactors and eliminate regulatory impediments to commercial interest in this technology, while ensuring adequate safeguards.

It is important that the private sector take the lead in developing commercial reprocessing services. Thus I am also requesting the director of the office of Science \& Technology Policy, working with the secretary of energy, to undertake a study of the feasibility of obtaining economical plutonium supplies for the Departinent of Energy by means of a competitive procurement. By encouraging private firms to supply fuel for the breeder program at a cost that does not exceed that of government-produced plutonium, we may be able to provide a stable market for private sector reprocessing, and simultaneously reduce the funding needs of the U.S. breeder demonstration program. 
4. I am instructing the secretary of energy, working closely with industry and state governments, to proceed swiftly toward deployment of means of storing and disposing of commercial high-level radioactive waste. We must take steps now to accomplish this objective and demonstrate to the public that problems associated with management of nuclear waste can be resolved.

5. I recognize that some of the problems besetting the nuclear option are of a deep-seated nature and may not be quickly resolved. Therefore, I an directing the secretary of energy and the director of the Office of Science \& Technology Policy to meet with representatives from the universities, private industry and the utilities and requesting them to report to me on the obstacles which stand in the way of increased use of nuclear energy and the steps needed to overcome them in order to assure the continued availability of nuclear power to meet America's future energy needs not later than Sept. 30, 1982.

El iminating the regulatory problems that have burdened nuclear power will be of little use if the utility sector cannot raise the capital necessary to fund construction of new generating facilities. We have already taken significant steps to improve the climate for capital formation with the passaye of my program for economic recovery. The tax bill contains substantial incentives designed to attract new capital into industry.

Safe, commercial nuclear power can help meet America's future energy needs. The policies and actions that I an announcing today will permit a revitalization of the U.S. industry's efforts to develop nuclear power. In this way, native American genius--not arbitrary federal policy--will be free to provide for our energy future. 


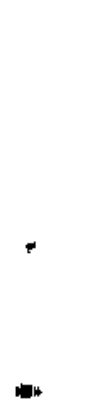

wa 


\section{APPENDIX G}

FINANCIAL ANALYSIS 
APPENDIX G

\section{FINANCIAL ANALYSIS}

A life-cycle cost analysis was done to determine the cost of installing 20 meter stacks on exhaust boreholes. The model that was developed considers only those incremental costs that are incurred as a direct result of the stacks. For example, the cost of existing ventilation would not be considered, but the increased cost of ventilation due to the addition of the stack would be included.

The analysis was done on a cash flow basis and a standard cash flow convention was used. All cash flows are assumed to occur at the end of the period except for capital investments which occur at the beginning of the period. For example, if the year 1983 is period 1 on the timeline below, then all capital investments made during 1983 are assumed to occur at time 0 and all other cash flows during the year as assumed to occur at time 1 . This is equivalent to saying that investments occur on January 1 st and all other cash flows (operating costs, taxes, etc.) happen on the last day of December.

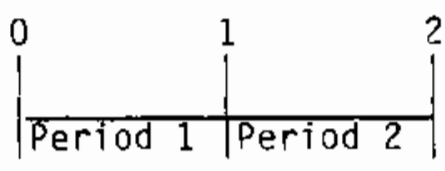

The reason for using a life-cycle cost analysis is that the results are analogous. Mines with different lifetimes and production rates are comparable under this method when real costs, as opposed to nominal costs, are used. The levelized cost that the analysis results in is the additional real dollar amount per unit of production that would have to be received to offset the added investment and operating costs as well as compensate the investors for the opportunity cost of their committed funds. This levelized cost is given in terms of dollars per ton of ore and doliars per pound of $\mathrm{U}_{3} \mathrm{O}_{8}$. 
This analysis can be done in different ways depending on its purpose. The production life can be considered as the full-life of the mine or the remaining life. Also the analysis can assume an inflation rate or no inflation.

- Full Life versus Remaining Life - If the levelized cost is derived over the full life of the mine, it is equivalent to saying that the stacks were in place at the beginning of the mine's life. Thus, the cost of the stacks is spread over the entire life of the mine, and the levelized cost is applied to every unit of production. Conversely, if the levelized cost is derived over the mine's remaining production life, this implies that the stacks are new and that the cost should only apply to future production. The full life and remaining life methods are equivalent when applied to new mines.

- Inflation versus No Inflation - Including inflation or real cost escalation in the analysis does not undermine the validity of the analysis, but it does make the results less easily understood. For example, on the time line below two cash flow streams are shown. The top stream is given in real dollars (inflation adjusted), and the bottom stream is nominal dollars (not adjusted for inflation).

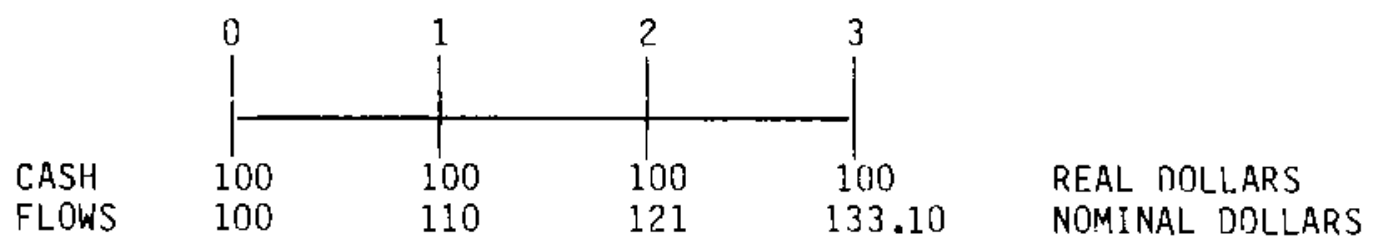

The resulting levelized cost for the real dollar stream is $\$ 100.00$. This means that to recover this cost, a charge of $\$ 100.00$ per period would be required (assuming no inflation); or a charge of $\$ 100.00$ per period in real terms (i.e., escalate the $\$ 100.00$ charge by the inflation rate). The levelized cost for the nominal dollar stream is $\$ 114.72$ assuming a discount rate equal to the escalation rate. If one charged $\$ 114.72$ per period, the cost would be recovered. The difference may seem small here, however, if there had been twenty cash flows instead of four the levelized cost of the uninflated stream would still be $\$ 100.00$, but the levelized 
cost of the inflated stream would be $\$ 213.56$. Thus, if the cost did escalate at $10 \%$ per year, and one charged $\$ 213.56$ per period, the cost would be fully recovered. This number is not easy to get an intuitive feel for, whereas $\$ 100.00$ per period in real terms is a number with more practical appeal.

The life-cycle cost analysis, as mentioned before, gives comparable results for mines with different lifetimes and production rates when real costs are used. For the results to be completely analogous, the mine lives must be of the same length if there are any costs escalating at a real rate or if inflation is included. In the example above when real costs were used, it did not make any difference if the time horizon was three of nineteen periods, the cost remained at $\$ 100.00$. However, when the nominal cost stream was used, the levelized costs were vastly different and not easily compared.

If the analysis is done assuming no inflation, then cash flows fixed in nominal terms tend to be over stated. For example, the value of the depreciation tax shield is greatest when the inflation rate is lowest. This causes the levelized costs to be stated slightly lower than their actual values, but still allows the results between mines to be easily compared. 


\section{LEVELIZED LIFE-CYCLE COST MODEL}

The model developed here is consistent with current DOE and EPRI requirements for evaluation methodologies, and it resembles the approach used by investor-owned utilities to estimate the cost of power from new sources (jet Propulsion Laboratory, 1976 and Electric Power Research Institute, 1982).

The intent of life-cycle costing is to find the price that all expected output would have to be sold to result in the exact revenue required to fully recover the costs over the mine's lifetime. These costs implicitly include a return on the investments of stockholders and creditors through the use of a discount rate equal to the firm's cost of capital.

In this model all costs are stated in first quarter 1983 dollars. The present value of these costs is then converted into an annuity over the production lifetime in the same manner as a home mortgage payment. This levelized annual cost is divided by the yearly production rate to give a levelized life cycle cost in terms of dollars per pound of $\mathrm{U}_{3} \mathrm{O}_{8}$ or dollars per ton of ore.

Specifically, the levelized annual cost is expressed mathematically by the following equation:

$$
L A C=\sum_{j=0}^{m}\left(F C R_{j}\right)\left(C I_{j}\right)+C R F(F P L+H O C)
$$

where FPL and HOC are the present values of the additional fan power costs, and heater operating costs, respectively. Each of these values is calculated using the following equation:

$$
P V(x)={ }_{i=1}^{N} \times\left(\frac{1+g_{x}}{1+k}\right)^{i}
$$

where $X$ is the estimated first year cost (in 1983 dollars) and $g_{x}$ is the appropriate escalation rate. The rest of the variables are defined later in the financial parameters section. The capital recovery factor is given by the following: 


$$
C R F=\frac{k}{1-(1+k)^{-N}}
$$

and it levelizes the present value of the annual operating costs. The present value of each capital investment (CI, in 1983 dollars) is multiplied by the fixed charge rate appropriate for the year of that investment. The equation for the fixed charge rate is given below:

$$
F C R=C R F\left[\frac{1-\tau(D P F)-T C}{1-\tau}\right]+T F+I F
$$

and the variables are defined in the following section.

\section{Financial Parameters}

For the life-cycle cost analysis, the following financial parameters have been determined or assumed:

- Constant Dollars - All levelized cost values will be expressed in constant 1983 dollars. Also, all other dollar values will be in 1983 dollars except where specifically noted.

- Size of Firm - It is assumed that the financial entity making the investments is profitable enough to take full advantage of the maximum allowable depreciation, amortization, and investment tax credits. It is also assumed that the firm is profitable enough to pay the maximum marginal corporate tax rates.

- Federal Tax Rate - The current maximum marginal federal corporate tax rate is $46 \%$.

- State Tax - A weighted average state income (or franchise) tax was used. The states and their respective maximum rates are: Colorado - 5\%, Utah $4 \%$, Wyoming - none, and New Mexico - 6\%. The weightings correspond to the number of active underground uranium mines. Specifically, the analysis will assume a $4.6 \%$ state income tax rate.

- $I=$ Effective Corporate Income Tax Rate - This rate is applied to gross profits to determine the corporate income tax liability. It takes into account the deduction from gross profits, of state incone tax liabjlities, when computing the federal tax liability. Specifically the value of $48.5 \%$ is used. 
- $I C=$ Federal Tax Credit - Investment tax credits will be $10 \%$ on all depreciable assets classified as five-year ACRS property.

- $D P F=$ Depreciation Factor - This value is the present value of the future depreciation charges as a fraction of the original value of the capital investment. Depreciation expenses will be computed according to the 1982 tax act. Specifically, new equipment eligible for the investment tax credit (heaters) will be depreciated using the five-year ACRS schedule for property placed in service between 1981-1984. As per the 1982 law revision, this schedule will be applied to $95 \%$ of the equipments original cost. Ventilation stacks are depreciable, but not eligible for the investment tax credit, will be depreciated according to the 10-year ACRS schedule at $100 \%$ of the cost of development. In any case in which the stack or heater life is longer than the mine life, the unit production method will be used. Here it will be assumed that production will remain level over the life of the mine. Thus, depreciation will be a straight line over the mine life for this situation. Depreciation will be assumed to be the same for state and federal income tax purpose.

- IF = "Other" Tax Fraction - This value is assumed to be $2 \%$ of the present value of the total capital investment. It represents all non-income annual taxes and license fees. These "other" taxes are assumed to be deductible for income tax purposes and, as an approximation, assumed to be constant.

- IF = Insurance Fraction - This value is assumed to be one-quarter of one percent of the present value of the total capital investment. As an approximation, this fraction is assumed to be constant.

- $g_{c}=$ Capital Cost Escalation Rate - This value is also assumed to be equal to the general inflation rate. The analysis will be done in two ways; with and without inflation. Specifically values of $0 \%$ and $5 \%$ will be used.

- $g_{E}=$ Real Escalation Rate for Energy Costs - This rate is to be added to the inflation rate to get the nominal electricity cost escalation rate. A value of $2 \%$ will be used in this study, which was the electricity cost 
escalation rate estimated by Brookhaven National Laboratory for the period 1975 to 1990 (Weissbrod and Barron 1979). This rate is to be applied to ventilation power costs and fuel costs for heater operation.

- $N=$ Mine Life in Years - This value can represent either the remaining years of production or the total mine life depending on the type of analysis being done.

- Salvage Value - All capital items are assumed to have zero salvage value at the end of their depreciable life. Equipment life is assumed to equal or exceed the mine life in all cases.

- $K=$ The Discount Rate - To determine the appropriate discount rate the Capital Asset Pricing Model was used. Discount rates for six of the major uranium producers were calculate and an average value of $5.73 \%$ (rea1) was found. The equity betas were calculated by Value Line Investment Survey and the historical market average was used for the debt betas. The method is shown below and historical values were used for the average market return and real riskless rate of return.

The Capital Asset Pricing Model

General equation for calculation of discount rates for projects of risk similar to the company's current business.

$$
r_{f}+B_{\text {Assets }}\left[E\left(r_{m}\right)-r_{f}\right]=E\left(r_{A}\right)=\begin{aligned}
& \text { Company cost of capital } \\
& \text { hurdle rate for capital- } \\
& \text { budgeting decisions. }
\end{aligned}
$$

where:

$$
\begin{aligned}
E\left(r_{A}\right)= & \text { Expected return on assets. } \\
r_{f}= & \text { Risk-free rate of return. } \\
E\left(r_{m}\right)= & \text { Expected return on the entire market portfolio (i.e., all } \\
& \text { assets) } \\
B_{\text {Assets }}= & B_{\text {Debt }}\left(\frac{\text { Debt }}{\text { Debt }+ \text { Equity }}\right)+\beta_{\text {Equity }}\left(\frac{\text { Equity }}{\text { Debt }+ \text { Equity }}\right) \\
B_{\text {Debt }=} & \text { Covariance of return on debt (company specific) with the } \\
\text { market return. } & \text { Covariance of return on equity (company specific) with the } \\
& \text { market return. A measure of volatility of the individual } \\
& \text { security relative to the market. }
\end{aligned}
$$


Debt = market value of the company's outstanding debt. Equity = Market value of the company's outstanding equity.

Since it is almost impossible to measure expectations, historical values are used as a proxy for the expected return on the market portfolio and the real risk-free rate of return. Historical data is also used to calculate an average corporate debt beta. This data was compiled by the Financial Analysts Research Foundation (Ibbotson and Sinquefield 1977). The rates of return listed below are for the period 1926 through 1976, and they are inflation adjusted geometric mean values.

$\begin{aligned} \text { Real Return on Long-term Government Bonds } & =1.0 \% \\ \text { Real Return on Long-term Corporate Bonds } & =1.7 \% \\ \text { Real Return on Common Stocks } & =6.7 \%\end{aligned}$

From the above data the following values can je calculated or surmised:

$\bar{r}_{f}=$ Average Real Riskless Rate of Return $=1.0 \%$

$E\left(r_{m}\right)=$ Expected Return on the Market Portfolio $=6.7 \%$

$\left(\overrightarrow{r_{m}-r_{f}}\right)=$ Average Market Risk Premium $=5.7 \%$

$E\left(r_{C B}\right)=$ Expected Return on Corporate Bonds $=1.7 \%$

$$
E\left(r_{C B}\right)=\bar{r}_{f}+\bar{\beta}_{\text {Debt }}\left(\overline{r_{m}-r_{f}}\right)=1.7 \%
$$

$B_{\text {Debt }}=$ Average Beta for Corporate Bonds $=0.1228$

For each of the six firms that were used in the calculation of the discount rate, the asset beta was first calculated using equation (G.6). As mentioned before, the equity betas were calculated by Value Line and the average beta for corporate bonds was used as a proxy for each firms debt beta. With the calculated asset beta and the values above, equation (G.5) was used to compute the discount rate for each firm and an average was taken.

The average discount rate computed above assumes no inflation and there is no assumption made as to the amount of debt. The discount rate is the minimum acceptable rate of return on an investment. To adjust this value for inflation, one simply adds to it the inflation rate. For this study, values being calculated assume zero or five percent inflation. Thus the discount rates would be $5.73 \%$ and $10.73 \%$ respectively. 
If this analysis was being done on the basis of invested equity capital, then a debt/equity ratio would need to be assumed. The appropriate discount rate would then be calculated using the following expression:

$$
E\left(r_{E}\right)=r_{f}+B_{\text {Equity }}\left[E\left(r_{m}\right)-r_{f}\right] \text {. }
$$

This will give equivalent results however. The return on invested equity capital will be greater than the return on assets and the return on the debt will be less. But the total return will be the same. Since the debt/equity ratio of the major uranium producers varies between about 0 and 1 , it is not fair to select a particular value. Thus to avoid this problem and still maintain the validity of the analysis, the return on assets method (Equation G.5) is used. 
PNL -5035

$U C-2,11,55$

\section{DISTRIBUTION}

No. of

Copies

OFFSITE

40 Jay Silhanek Environmental Protection Agency Office of Radiation Programs washington, D.C. 20460

27 DOE Technical Information Center
No. of

Copies

ONSITE

DOE Richland Operations office

HE Ransom

32 Pacific Northwest Laboratory

G.A. Bruno (15)

J.A. Dirks

P.0. Jackson

J.K. Young

C.H. Bloomster

R.M. Fleischman

Publishing Coordination (2)

Technical Information (5)

Energy Systems Library (5) 
4

?

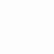

